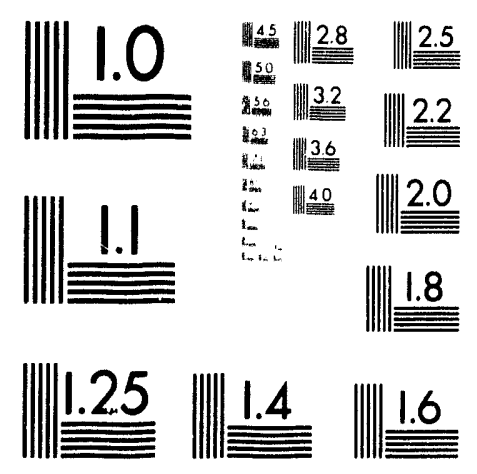



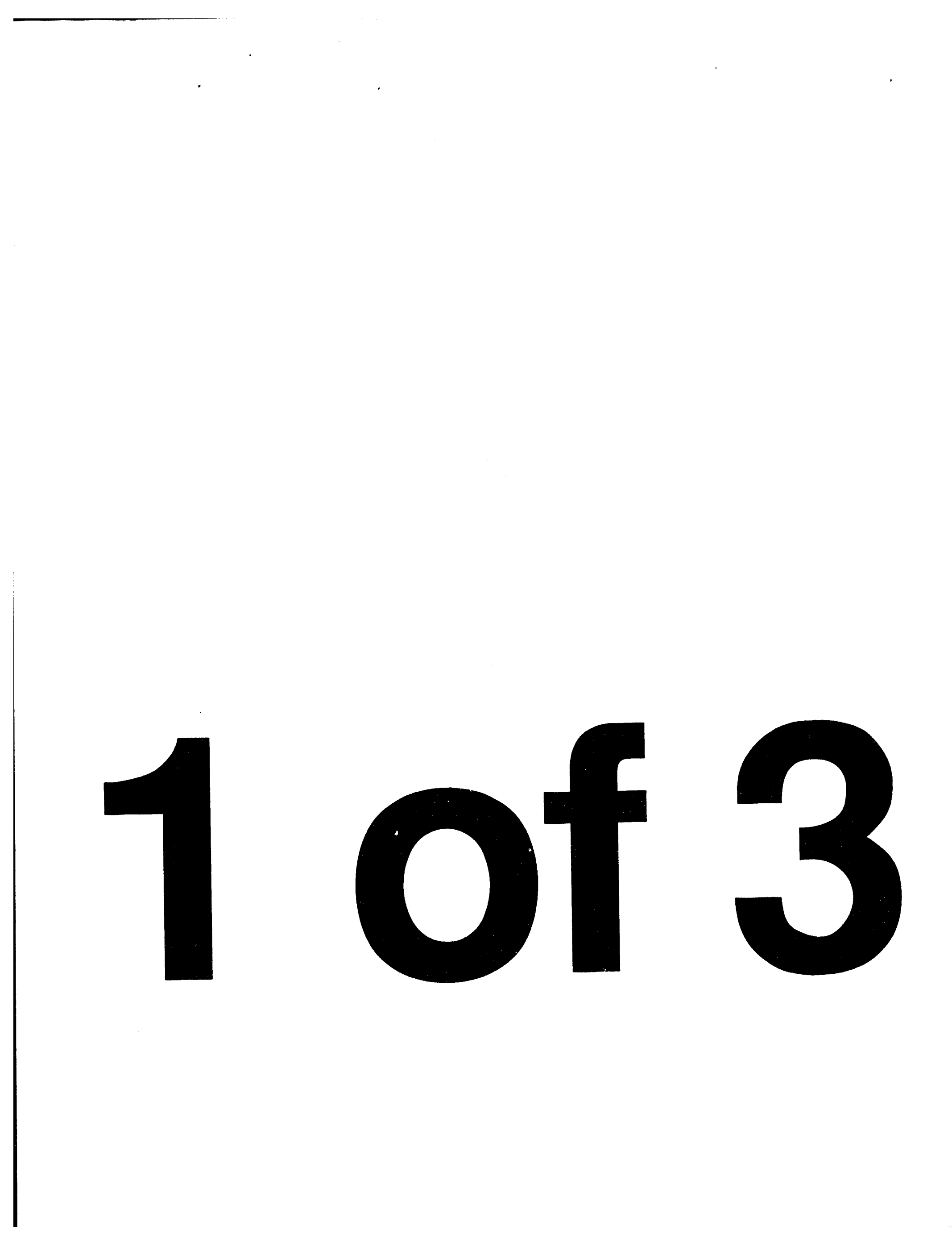
LBL-34712

UC-413

\title{
Disassembly of Hot Nuclear Matter Formed in Au- induced Reactions Near the Fermi Energy
}

\author{
Dimitrios Nicholas Delis \\ Ph.D. Thesis
}

\author{
Department of Chemistry \\ University of California \\ and \\ Nuclear Science Division \\ Lawrence Berkeley Laboratory \\ University of California \\ Berkeley, California 94720
}

September 1993

This work was supported by the Director, Office of Energy Research, Division of Nuclear Physics of the Office of High Energy and Nuclear Physics of the U.S. Department of Energy under Contract DE-AC03-76SF00098 


\begin{abstract}
Complex fragment emission has been studied in the $60 \mathrm{MeV} / \mathrm{A}{ }^{197} \mathrm{Au}+{ }^{12} \mathrm{C},{ }^{27} \mathrm{Al}$, ${ }^{51} \mathrm{~V}$, nat $\mathrm{Cu}$, and ${ }^{197} \mathrm{Au}$ reactions. Velocity spectra, angular distributions and cross sections have been constructed for each target from the inclusive data. Coincidence data including 2-, 3-, 4-, and 5-fold events have also been examined. Furthermore neutron multiplicity distributions have been obtained for the above reactions by utilizing a novel neutron calorimetric approach.

In the $60 \mathrm{MeV} / \mathrm{A}{ }^{197} \mathrm{Au}+{ }^{12} \mathrm{C}$ reactions, the complex fragments are produced mainly in highly equilibrated binary processes; in particular, they arise from the compound binary decay of Au-like nuclei. The relaxed nature of the decay process has been determined by the angular distributions and emission velocities of the fragments. The binary nature of the decay process has been illustrated by the well defined Coulomb rings and by the 2 -fold coincidence events. Higher $n$-fold events $(n=3$, and 4$)$ have also been observed but are less than $2 \%$ of the total coincidence events.

In the $60 \mathrm{MeV} / \mathrm{A}{ }^{197} \mathrm{Au}+{ }^{27} \mathrm{Al},{ }^{51} \mathrm{~V},{ }^{63} \mathrm{Cu}$, and ${ }^{197} \mathrm{Au}$ reactions the flat angular distributions of the fragments along with their Coulomb like emission velocities suggest the presence of a strong compound binary component in the inclusive data. This component is associated with the compounu nucleus decay of Au-like projectiles generated in peripheral collisions and dominates the singles cross-sections. However, in these reactions a significant yield of fragments arises also from multifragmentation. Charge distributions, velocity distributions, $Z_{1}-Z_{2}$ plots, where $Z_{1}$ and $Z_{2}$ are the charges of the fragments in the binary events, and $Z_{\text {total }}-V_{\text {source }}$ plots, where $Z_{\text {total }}$ and $V_{\text {source }}$ are the total charge and velocity of the source, have been constructed from the
\end{abstract}


coincidence events; these plots suggest that multifragment emission processes become increasingly important for the more symmetric entrance channels. Furthermore the relative probabilities for the multifold events have been found to increase substantially with excitation energy $E^{*}$. The excitation energy $E^{*}$ is determined, within the incomplete-fusion model, from the source velocity $\mathrm{V}_{\text {source. }}$. These probabilities are independent of the target-projectile combination, indicating that the dynamics of the reaction may be limited to the formation of a source through a mechanism similar to incomplete fusion.

The neutron multiplicity is utilized in the present experiment as an independent measure of the deposited excitation energy; in peripheral reactions one observes low neutron multiplicities associated with low excitation energies, while the more central collisions are associated with high neutron multiplicities and high excitation energies.

Possible mechanisms for multifragmentation are investigated by comparing some theories with the experimental data. The $60 \mathrm{MeV} / \mathrm{A} \mathrm{Au}$-induced reactions are simulated by coupling a kinetic description of the dynamical stage of the collision with a subsequent statistical decay of the primary sources. Results obtained with this model are shown and are compared with both inclusive and exclusive experimental data.

In a different approach, the possibility that the system undergoes a phase transition (percolation-like or liquid vapor-like) is investigated by examining observables that behave qualitatively differently whether a phase transition is present or not. These quantities have been constructed from the event-by-event moments of the fragment charge distributions. Possible signals of a phase transition have been extracted from the data.

Finally a characteristic energy dependence of the multifragment decay probabilities of the source or sources is presented similar to that observed for fission probabilities at low energies. This behavior seems to relegate the role of dynamics to the formation of the sources, which then proceed to decay in an apparently statistical manner. 
To my parents

Nicholaos and Stavroula 


\section{Acknowledgements}

In this section I would like to take the opportunity to thank some of the people that have contributed, although in different ways, in helping me get through graduate school and shaping in general my outlook.

First I would like to express my gratitude to my dissertation advisor, Prof. Luciano Moretto, for his guidance, support, and the numerous discussions we had in science and in history during my stay in Berkeley. Prof. Moretto's intellectual prowess has been a source of inspiration and guidance. Even when we disagreed, his critiques were instrumental in tempering my outlook. Furthermore I would like to thank him for his persistent efforts in improving my writing skills as can be seen from this thesis.

Second I would like to thank Dr. Gordon Wozniak for his encouragement, friendship, and technical help during various phases of my work. Dr. Wozniak showed me the importance of teaching patiently, without talking down, and without treating the student as if he was the village idiot.

In addition I am grateful to the following people who have been helpful with this project: Karl Hanold and Nicola Colonna. Thanks also goes to the rest of the members of the Morreto/Wozniak group, past and present, for their help in various phases of this project.

But most of all I would like to thank my parents, Nicholaos and Stavroula, my sister Hareclea and my brother Steve for the love and moral support they have given me throughout my graduate studies. I believe that if it was not for their encouragement I would have given up a long time ago. 


\section{Table of contents}

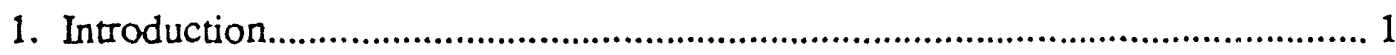

1.1 Complex Fragment Emission in E/A $<30 \mathrm{MeV} / \mathrm{A}$ reactions......................... 2

1.2 Complex Fragment Emission in $30<\mathrm{E} / \mathrm{A}<100 \mathrm{MeV} / \mathrm{A}$ reactions...............5

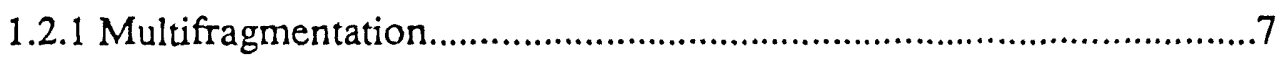

1.2.2 Experimental Variables and Observables.............................................8

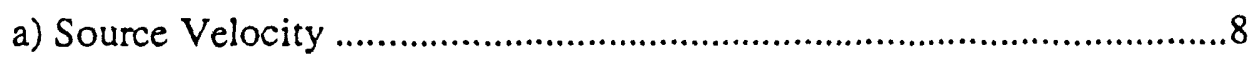

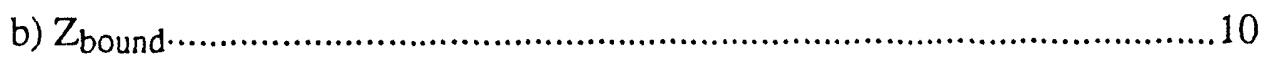

c) Particle - Particle Correlations...............................................................11

d) Fragment - Fragment Correlations ……………………......................13

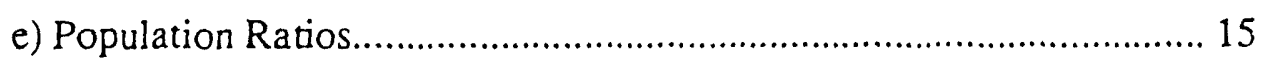

f) Light Particle Multiplicity.................................................................17

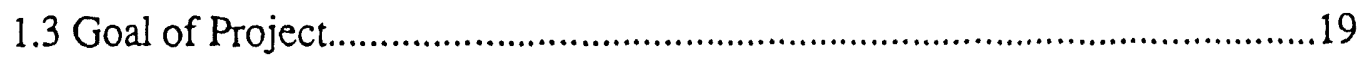

2. Theory

2.1 Origin of Complex Fragments in Compound Nucleus Reactions...................23

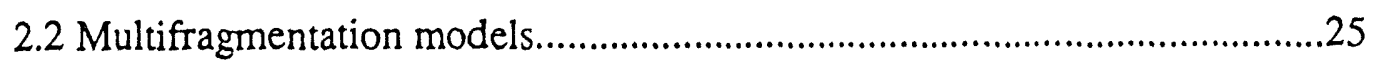

2.2.1 Statistical Theories..........................................................................26

a) Sequential Binary Decay...............................................................26

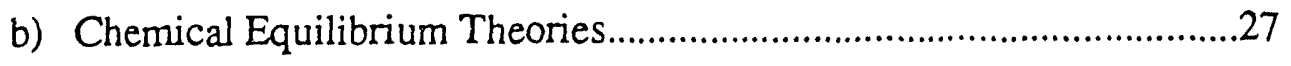

Liquid Vapor Equilibrium................................................................27

Canonical/Microcanonical Approximations.......................................28

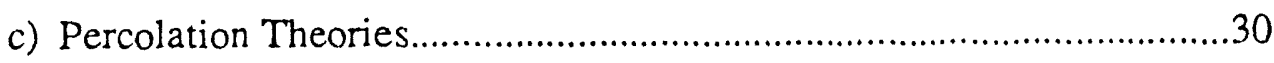




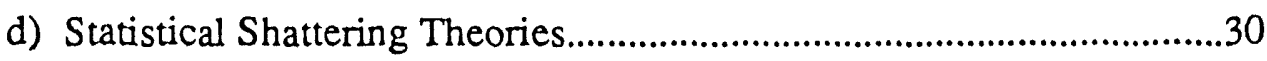

2.2.2 Dynamical Theories.............................................................................32

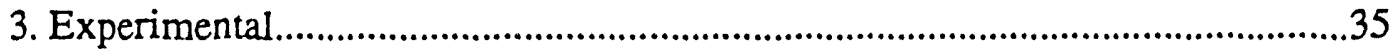

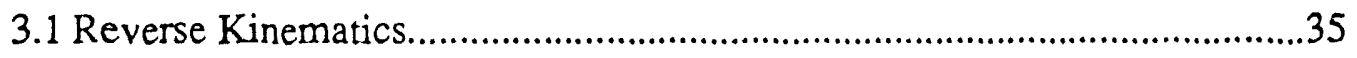

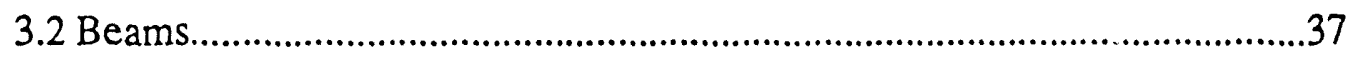

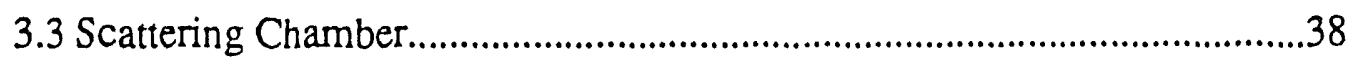

3.4 Beam Current Monitor....................................................................................

3.5 Scintillator Paddle or Active Collimator.........................................................38

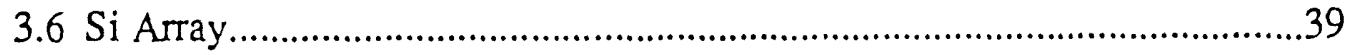

3.7 Neutron Calorimeter.................................................................................

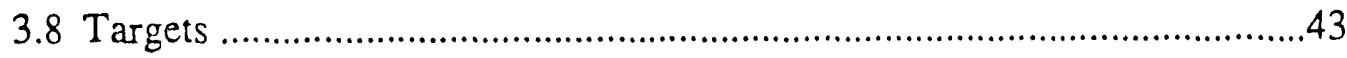

3.9 Electronics and Data Acquisition.............................................................44

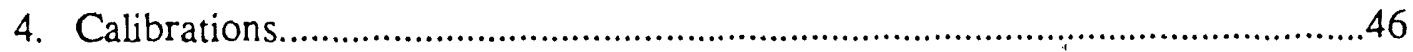

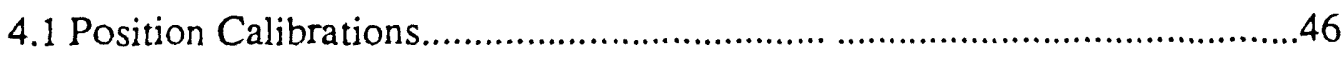

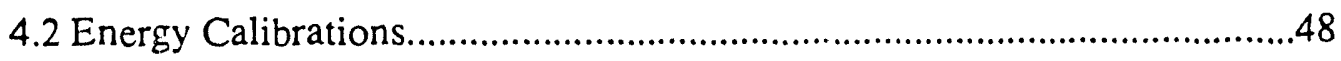

4.3 Atomic Number Calibrations and Mass Parameterization ...........................50

Atomic Number Calibrations.....................................................................50

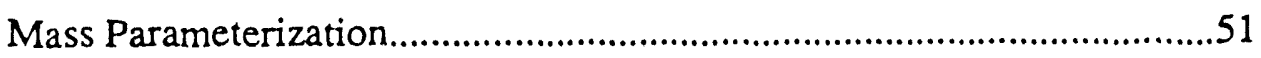

4.4.1 Neutron Calorimeter Calibrations................................................................52

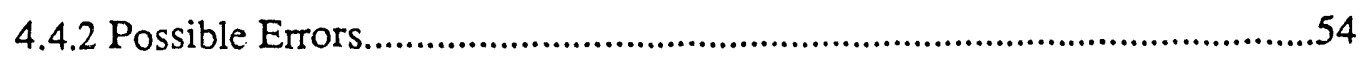

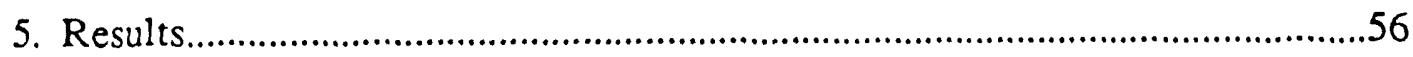

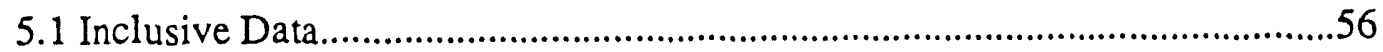

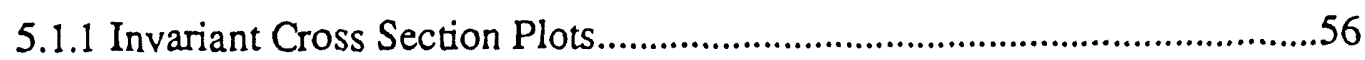

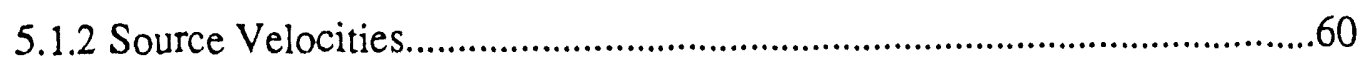

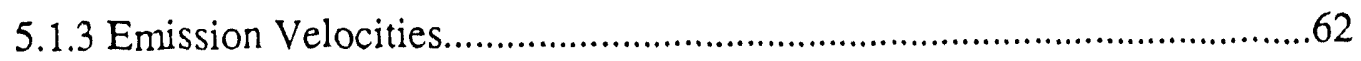




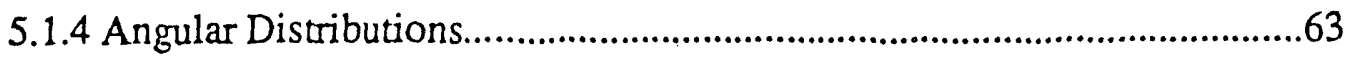

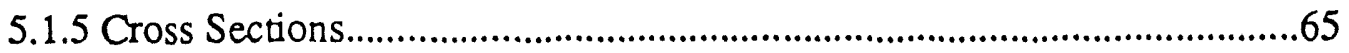

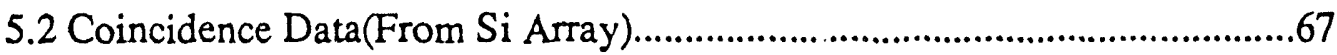

5.2.1 Z-distributions Selected by Fragment Size...............................................67

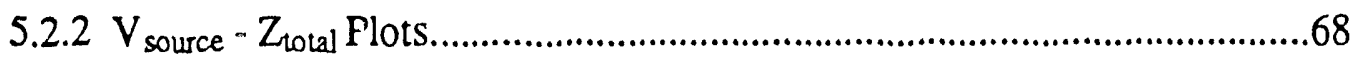

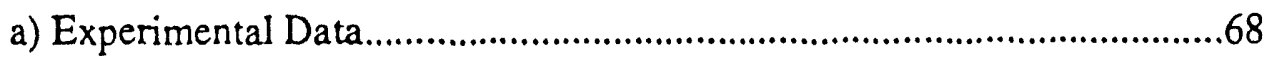

b) Possible Interpretation...........................................................................70

5.2.3 $\mathrm{Z}$ and $\mathrm{V}$ distributions according to fragment multiplicity..........................71

a) Z-distributions.........................................................................................

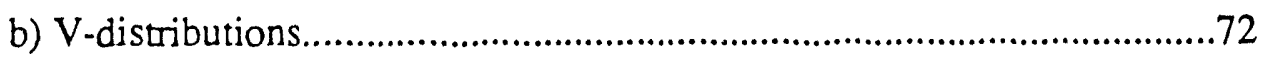

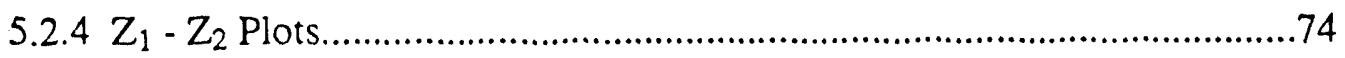

5.2.5 Excitation Functions..........................................................................75

5.2.6 Efficiency Corrected Excitation Functions...............................................78

5.2.7 Fragment Distributions Gated by Source Velocity....................................82

5.2.8 Relative Abundance of Fragments as a Function of Excitation Energy....84

5.3 Coincidence Data with the Neutron Detector....................................................86

5.3.1 Neutron Multiplicity Distributions...........................................................86

5.3.2 Fragment Distributions Gated by Neutron Multiplicities.............................87

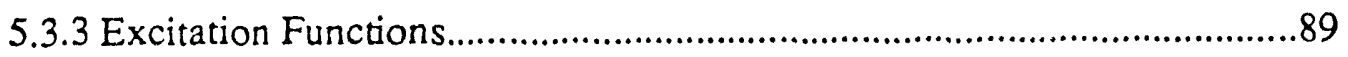

5.3.4 Excitation Energy vs Neutron Multiplicity.................................................90

6. Discussion and Calculations............................................................................93

6.1 A Hybrid Model Approach:(dynamical + statistical)......................................93

6.1.1 Codes and Calculations.............................................................................94

6.1.2 Comparisons with Experimental Data.......................................................101

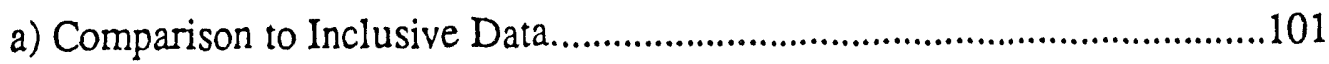

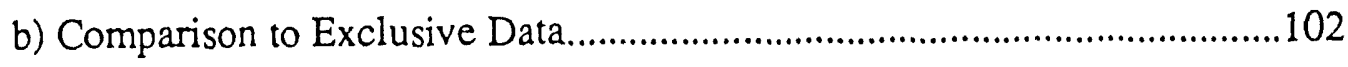

6.2 Phase transition? 


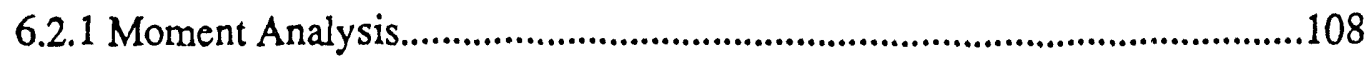

6.3 Statistical Multifragmentation?...................................................................114

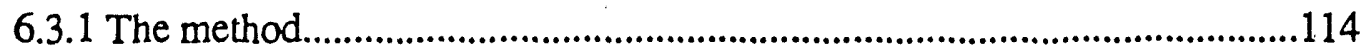

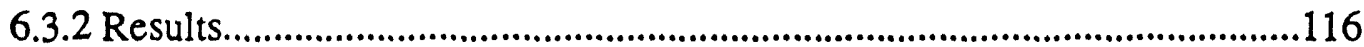

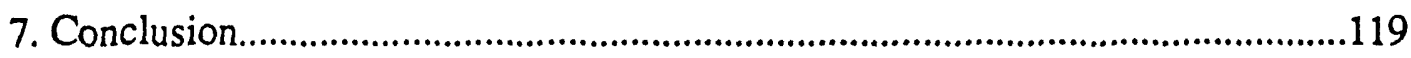

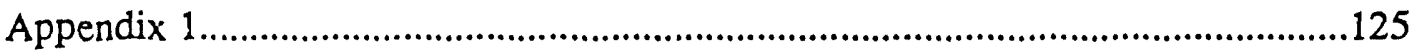

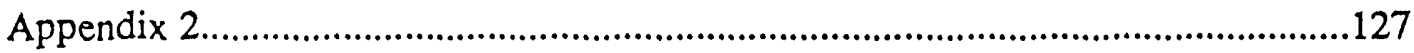

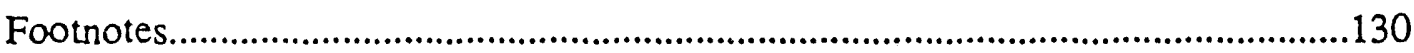

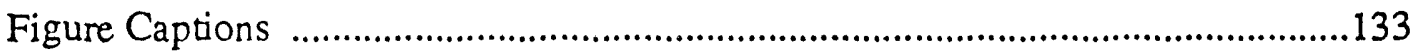

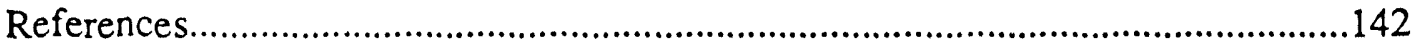

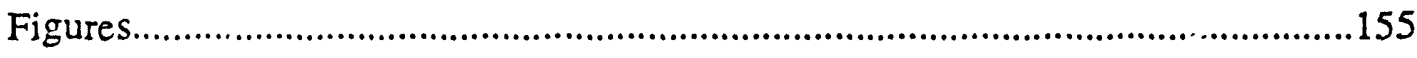




\section{Chapter 1}

\section{Introduction}

The collision between two nuclei can lead to the production of complex fragments. Complex fragments (CF) or intermediate mass fragments (IMF) as they are also commonly referred to are classified as those reaction products whose mass falls between ${ }^{4} \mathrm{He}$ and fission fragments. They were first identified in the early $1950 \mathrm{~s}$ [Mi 53, Fri 54, Ca 58] in radiochemical studies with high-energy protons bombarding medium to heavy targets. Later, counter experiments were performed at the Lawrence Berkeley Laboratory Bevatron and BEVALAC [Po 71, Hy 71] in which reaction products were measured over a large range of atomic numbers (up to $Z \sim 20$ ). Since then the emission of complex fragments has been observed in nucleus-nucleus collisions over a large range of bombarding energies [Ko 73, Go 77, Me 80, Bo 84, So 84, Mc 85, Fi 86, Ch 88a, Ch 88b, Ch 90, Rou 93].

In low bombarding energy $(\mathrm{E} / \mathrm{A}<15 \mathrm{MeV} / \mathrm{A})$ reactions the production of complex fragments is essentially associated with binary decay processes; such processes include quasi-elastic/deep inelastic and compound nucleus reactions (see section 1.1). The

production of complex fragments from the binary decay of a compound nucleus has been verified also in the 15-30 MeV/A bombarding energy regime (see section 1.1). At 
intermediate bombarding energies $(30<\mathrm{E} / \mathrm{A}<100)$ however, the reaction mechanism(s) governing the production of complex fragments are less clear. By increasing the bombarding energy, the binary signature of a compound nucleus decay disappears and an increasing yield of three-body and higher-order events is observed; this process in which several fragments are produced in the exit channel has been labeled multifragmentation and will be the central theme of this work.

In this chapter we will initially (section 1.1) discuss the emission of complex fragments at bombarding energies $\mathrm{E} / \mathrm{A}<30 \mathrm{MeV} / \mathrm{A}$. The processes governing the production of complex fragments in this energy regime are well understood and have been established in several experimental studies. Subsequently, in section 1.2, we will talk about the process of multifragmentation. A brief summary will be given of some of the major experimental efforts that have been put forth in order to understand multifragmentation. Finally, in section 1.3, we will present the goals of this thesis.

\subsection{Complex Fragment emission in E/A $<30 \mathrm{MeV} / \mathrm{A}$ reactions.}

In low-energy reactions $(\mathrm{E} / \mathrm{A}<15 \mathrm{MeV} / \mathrm{A})$ two sources of complex fragment emission have been established: quasi-elastic/deep inelastic and compound nucleus processes [ Kau 59, Gal 70, Mor 73, Kra 74, Sch 77, Mor 81, Mc 85, De 90, De 91]. In quasi-elastic/deep inelastic reactions complex fragments are produced as the binary decay products of transient dinuclear systems originating from the target-projectile combination. Most of the experimental information concerning deep-inelastic collisions has been obtained by measuring the kinetic energies, charge, mass, and angular distributions of the final reaction products [Kau 59, Gal 70, Mor 73, $\operatorname{Kra} 74, \operatorname{Sch} 77$, Mor 81]. In these reactions, the final kinetic energies of the fragments display various degrees of damping of the entrance channel kinetic energy, ranging from Coulomb-like 
interaction energies (in completely damped reactions) to essentially elastic energies (in quasi-elastic scattering). The mass distribution of the fragments is characterized by two broad peaks that can be associated with target and projectile like fragments; the broad distributions associated with these peaks depend on the exchange of nucleons that occurs during the interaction between the two nuclei. The mass transfer process is sensitive to both the interaction time and the potential energy of the intermediate dinuclear system. Furthermore, depending upon the interaction time, the observed angular distributions can be either forward peaked (projectile-like), backward peaked (target-like), or side peaked, indicating that the interaction time is typically shorter than the rotational period of the di-nuclear system. In general, the fragments produced in low-energy deep-inelastic reactions can be classified as either projectile-like or targetlike.

In compound nucleus reactions the mechanism responsible for the production of complex fragments is well understood and will be discussed in some detail in section 2.1. Complex fragments arise from the characteristic binary decay of a compound nucleus. The emission of complex fragments from a compound nucleus has been established in several studies by measuring the excitation functions of the emitted fragments[Mc 85, De 90, De 91]. As an example, we present below some results from the experimental study done by Delis et. al. [De 91].

In this experiment [De 91] complex fragments emitted from a compound nucleus $\left({ }^{75} \mathrm{Br}\right)$ in the 5.0,6.2, 6.9, 8.0, 10.2, and $12.7 \mathrm{MeV} / \mathrm{A} \mathrm{Cu}+\mathrm{C}$ reactions were measured throughout the entire mass asymmetry range. The fragments were emitted from the compound nucleus ${ }^{75} \mathrm{Br}$ whose excitation energy ranged from $50 \mathrm{MeV}$ to $127 \mathrm{MeV}$, the lower limit being barely $15 \mathrm{MeV}$ above the highest barriers. The excitation functions of fragments with $\mathrm{Z}$ between $5<\mathrm{Z}<26$ for the $\mathrm{Cu}+\mathrm{C}$ reactions are shown in Fig. 1.1 and are reminiscent of fission excitation functions. The sharp rise of the cross section as 
function of increasing excitation energy demonstrates that the fragments originate from $\mathrm{CN}$ decay. The solid lines are calculations based on the compound nucleus theory (described in Chapter 2 and Appendix 1) and are in excellent agreement with the experimental points. The binary nature of the decay was confirmed by the coincidence data; practically all of the coincidence events consisted of two fragments with atomic numbers $Z_{1}$ and $Z_{2}$ whose sum $Z_{1}+Z_{2}$ was equal to the atomic number of the compound nucleus $Z_{c n}$.

Along with establishing the compound nucleus nature of the process, these studies [Mc 85, De 91] also explained the abundance of complex fragments at higher energies as contrasted to their extreme rarity below $10 \mathrm{MeV} / \mathrm{A}$ bombarding energy. The emission of complex fragments from a compound nucleus is a process that is associated with high barriers and it takes a substantial amount of excitation energy before it becomes a readily available channel (i.e. characterized by high cross-sections). As can be seen from Figure 1.1 it is necessary to deposit a few hundred $\mathrm{MeV}$ of excitation energy into compound nuclei before $\mathrm{CF}$ emission saturates.

The $\mathrm{CN}$ emission has been verified also in the 10-30 MeV/A bombarding energy regime [Ch 88a, Ch 88b, Ch 90, Co 89]. In this new regime a composite system results from fusion between the larger partner and the occluded piece of the smaller partner (this is called incomplete fusion); the occluded piece decreases in size with increasing impact parameter. The product resulting from incomplete fusion can relax into a compound nucleus. For fragments intermediate in mass between the projectile and target, the cross-sections seem to originate solely from the binary decay of equilibrated compound nuclei. This conclusion has been reached from the analysis of the intermediate velocities, (isotropic) angular distributions, direct measurements (coincidence events) of the binary nature of the process and above all from the shape and magnitude of the charge distributions as a function of excitation energy. 
For completeness, we mention that in this energy range (15-30 MeV/A) deep inelastic processes have also been established. The emission of complex fragments in the reaction $27 \mathrm{MeV} / \mathrm{A} \mathrm{Ar}+\mathrm{Ag}$ [Bor 88] was in fact associated with binary quasi- and deep-inelastic reactions. Furthermore deep inelastic reactions have been observed for the heavy symmetric system ${ }^{100} \mathrm{Mo}+{ }^{100} \mathrm{Mo}$ at both $\mathrm{E}=18.7$ and $23.7 \mathrm{MeV} / \mathrm{A}[\mathrm{Olm}$ 87].

\subsection{Complex Fragment emission in $30<E / A<100$ reactions.}

At intermediate bombarding energies $(30<\mathrm{E} / \mathrm{A}<100)$ the reaction mechanism(s) governing the production of complex fragments are less clear. By increasing the bombarding energy, the binary signature of a compound nucleus decay (or deep inelastic reactions) disappears and an increasing yield of three-body and higher order events is observed. These multifragment events can be explained by the fact that the primary binary decay products may be also very excited, and have a significant probability of decaying in turn into two additional fragments. However other explanations have been put forth.

Early investigations showed that in the region of $2<\mathrm{Z}<20$ the charge distributions could be described in terms of a power law $\mathrm{P}(\mathrm{Z}) \propto \mathrm{Z}^{-2.6}$ [Chi 83, Fie 84, Lyn 82, So 83]. Since a power law distribution is predicted for droplets of liquid in equilibrium near the critical temperature, this experimental evidence was taken as a signature of liquid-vapor equilibrium near the critical point. But the dependence observed in the charge distribution is not unique to a liquid-gas phase transition.

A variety of models, ranging from statistical to dynamical theories, have been proposed that reproduce the charge distribution dependence. Such models include e.g. cold fragmentation models [Boh 83, Huf 83, Aic 84], in which nuclei are assumed to 
break up on impact and shatter like brittle material; hydrodynamical models [Be 88, Sch 89 , Aic 91, Gr 87], in which the reaction is simulated by utilizing the collisionless Vlasov equation augmented by a two body collision term; percolation models [Bau 85 , Bau 86, Ngo 90, Cam 87b]; in which the nucleus is treated as a three dimensional lattice of nucleons connected by bonds. By increasing the excitation energy more bonds break and the system evolves from one large cluster to many small clusters of nucleons; and finally statistical multifragmentation theories; in which fragments are emitted statistically from a nucleus. A more detailed description of these models will be given in Chapter 2.

For the sake of completeness we mention that at high energies (approximatelly E/A $>100 \mathrm{MeV}$ ) the production of complex fragments can be explained by the fireball model [Gos 77, Wes 76]. In this model, unlike what happens in incomplete fusion, where the larger partner picks up the occluded piece of the smaller partner, nuclear matter is divided into three pieces - the projectile spectator, the target spectator, and the fireball that arises from the region in which the nucleons in the target and projectile overlap. The thermal energy per nucleon in the fireball (piece) is much larger than the nucleon binding energy, and it is likely that the fireball disassembles completely into nucleons. On the other hand, the spectators are characterized by a small amount of excitation energy that is proportional to their excess surface. In the fireball model complex fragments are emitted from the decay of the spectators.

It is possible that the production of complex fragments may be the result of a smooth evolution from reaction mechanisms established in low-energy reactions, such as deep inelastic/incomplete fusion/compound nucleus, to those associated with highenergy reactions $(E / A>100)$ like the participant-spectator (fireball) model. A diagram illustrating the approximate domains of the various processes as a function of bombarding energy is shown in Figure 1.2. The boundaries of these processes have not 
been clearly established and depend not only upon the impact parameter but also on the entrance-ck innel mass asym.metry [Mor 93].

\subsubsection{Multifragmentation}

Multifragmentation is defined as the break up of the nuclear system into several fragments and has been clearly established in numerous experiments [Bo 89, $\mathrm{Ki} 89, \mathrm{Tr}$ 89, B1 91, Bo 91b, Og 91, Bl 91, Ha 92, Rou 93].

Early evidence that nuclei break up into several pieces was provided by emulsion experiments [ $\mathrm{Ja} 82, \mathrm{Wa} 85$ ]. Jakobson et al. [ $\mathrm{Ja} 82$ ], by utilizing a ${ }^{12} \mathrm{C}$ beam of 852 $\mathrm{MeV}$, observed events with several tracks in the emulsion, that were associated with medium mass fragments. The absence of a big residue in these events suggested the complete breakup of the system.

Evidence that hot nuclei break up into several pieces has also been provided by numerous electronic experiments [ $\operatorname{Tr} 87, \mathrm{Bou} 88, \mathrm{Bo} 89, \mathrm{Ki} 89, \operatorname{Tr} 91, \mathrm{Bl}$ 91, Bo 91b, Og 91, $\mathrm{Ha}$ 92]. As an example, the authors of [Ji 88] have observed in the $\mathrm{Ar}+\mathrm{Al}$ reaction events with a multiplicity ranging from 1 to 5 fragments. The reaction was studied at several bombarding energies ranging from 25-45 MeV/A and a strong increase in the cross section for the production of events with 3-5 intermediate mass fragments (IMF) was observed around $35 \mathrm{MeV} / \mathrm{A}$. This suggests that multifragmentation may become an important decay channel at bombarding energies larger than $35 \mathrm{MeV} / \mathrm{A}$ for this particular system. In another study, a multiplicity as large as 15 fragments per event, has been observed by the authors of [ $\mathrm{Bal} \mathrm{91]}$ in the reaction $29.2 \mathrm{MeV} / \mathrm{A}{ }^{209} \mathrm{Bi}+{ }^{136} \mathrm{Xe}$. The atomic number of the smallest fragment in this study was $Z=4$. 
Unlike compound nucleus decay which is a well characrerized process, multifragmentation is poorly understood. Efforts to characterize the process of multifragmentation have been largely based on comparisons between theoretical and experimental variables or observables. Although theoretically it is easy to choose the variables that are most useful in describing multifragmentation, experimentally the situation is more complicated. This is because of the difficulty in directly determining these variables, and sometimes by the fact that different theories suggest different variables. In the following section we present selected examples of experimental work in which different variables or observables have been utilized in order to characterize multifragmentation.

\subsubsection{Experimental Observables and Variables}

Some of the variables (or observables) that have been used to identify the process of multifragmentation include:

a) the velocity $V_{S}$ of the multifragmentation source (m.s.)

b) the charge $\mathrm{Z}_{\text {bound }}$ of the multifragmentation source

c) particle-particle correlations

d) fragment-fragment correlations

e) population ratios

f) light particle multiplicities

Examples of experiments in which these variables have been utilized are presented below.

\section{a) Source Velocity}


A variable of considerable interest that has been utilized successfully to study compound nucleus decay [Han 89, De 91] is the velocity of the source from which the two complex fragments are emitted. The sou-ce velocity is given by the following expression:

$$
\mathrm{V}_{\mathrm{s}}=\frac{\Sigma \mathrm{m}_{\mathrm{i}} \mathrm{v}_{\mathrm{i}}}{\Sigma \mathrm{m}_{\mathrm{i}}}
$$

where $m_{i}$ and $v_{i}$ are respectively the mass and the velocity in the laboratory frame of the $\mathrm{i}$-th fragment, and the summation is performed over the two detected fragments.

Equation 1.1 has also been successfully used to identify and characterize binary sources formed in incomplete fusion reactions [ $\mathrm{Ch} 88 \mathrm{a}, \mathrm{Ch} 88 \mathrm{~b}, \mathrm{Ch} 90, \mathrm{Co} 89$ ]. In this case $V_{B}$ is constructed from the fragments emitted from the compound nucleus formed in the incomplete fusion process. The fragment(s) arising from the target remnant is(are) not included in the construction of $\mathbf{V}_{\mathbf{s}}$.

In a very recent study, the authors of [Ch 92] have isolated and characterized what appear to be true multifragmentation sources formed in the $35,40,45$, and $55 \mathrm{MeV} / \mathrm{A}$ $\mathrm{La}+\mathrm{C}, \mathrm{Al}, \mathrm{Ca}, \mathrm{Cu}$, and $\mathrm{La}$ reactions, by using the same technique that is used to identify binary sources in incomplete fusion reactions. In this case the summation in equation 1.1 was done over all detected fragments. It was found that the sources were formed through an incomplete fusion process and underwent multifragment decay in a way that was independent of the formation process. Furthermore it was shown that the observed probabilities for $2,3,4$, and 5 -fold events depended almost exclusively upon the excitation energy of the fused object, and little upon the target-projectile combination or bombarding energy. The excitation energy was determined from the reconstructed source velocity within the incomplete fusion $\operatorname{model}^{1}$ (such upper script 
numbers whenever they appear in the text refer to the FOOTNOTES section at the end of the thesis).

\section{b) $\mathbf{Z}_{\text {bound }}$}

A variable of considerable interest that has been used by Kreutz et. al. [Kre 92] to study the emission of complex fragments is the sum $\mathrm{Z}$ bound of the charges bound in the emitted fragments (with $\mathrm{Z} \geqq 2$ ). There should exist a correlation between this variable and the excitation energy, since large excitation energies would decrease $Z$ bound by light particle evaporation.

Kreutz et. al. [Kre 92] have studied the multi-fragment emission observed in the 600 $\mathrm{MeV} / \mathrm{A} \mathrm{Au}+\mathrm{C}, \mathrm{Al}, \mathrm{V}$, and $\mathrm{Pb}$ reactions by examining a series of observables as a function of $\mathrm{Z}$ bound. Some of these observables are: the average largest charge $<\mathrm{Zmax}>$; the average intermediate mass fragment (IMF) multiplicity $<\mathrm{M}_{\mathrm{IMF}}>$; the average value of the relative asymmetry between the two largest fragments $<A_{2}>$ etc...

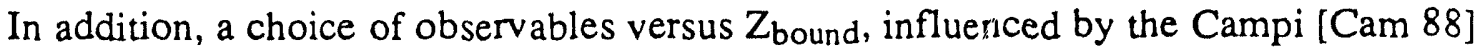
application of percolation theories to finite systems like nuclei, was also utilized.

One of the striking results of this study is that all quantities show a behavior which is independent of the target when plotted versus $Z_{\text {bound. This }}$ indicates that the decay of the system is independent of how it is formed.

Furthermore the experimental results presented in [Kre 92] were compared to different statistical model calculations. The results obtained by the models differed significantly from each other, establishing that such observables are sensitive to how the available phase space is populated and can be used to discriminate between models that have different treatments of nuclear disassembly. For instance, the sequential statistical model GEMINI predicted decays that were too asymmetric compared to the data, while the simultaneous statistical model predicted decays that were too symmetric. 
On the other hand the percolation model, which was adjusted to reproduce the average largest charge $<Z \max >$ and the average intermediate mass fragment multiplicity $<\mathrm{M}_{\mathrm{IMF}}>$ predicted correctly the remaining dependences.

\section{c) Particle - Particle Correlations.}

In Astronomy, stellar sizes can be determined by utilizing the two photon intensity interferometry technique developed by Hanbury Brown et al. [H↔n 56]. In this technique one measures the two photon correlation function for incoming coincident photons as a function of their relative momentum. The correlation function is given by

$$
\mathrm{R}\left(\mathrm{k}_{1}, \mathrm{k}_{2}\right)=\frac{\left\langle\mathrm{n}_{12}\right\rangle}{\left\langle\mathrm{n}_{1}\right\rangle\left\langle\mathrm{n}_{2}\right\rangle}-1
$$

where $\left\langle\mathrm{n}_{12}\right\rangle$ is the probability of detecting two coincident photons of wave numbers $k_{1}$ and $k_{2}$ in detectors 1 and 2 , and $\left\langle n_{j}\right\rangle$ is the probability of detecting a photon of momentum $k_{j}$ in detector $j(j=1,2)$. The two photons have a nonzero correlation function because of the symme rization of their wave functions; this is a consequence of quantum statistics for identical particles. To make this point clear consider the simplified case of simultaneous photon emission from two distant point sources located at $\mathrm{r}_{\mathrm{a}}$ and $\mathrm{r}_{\mathrm{b}}$. Assuming propagation in vacuum, the coincidence probability is proportional to the symmetrized two photon wave function given by:

$$
\begin{aligned}
\mathrm{n}_{12} & \sim\left|\exp \left(i \mathbf{k}_{1} \mathrm{r}_{\mathbf{a}}+i \mathbf{k}_{2} \mathrm{r}_{\mathbf{b}}\right)+\exp \left(i \mathbf{k}_{1} \mathrm{r}_{\mathbf{a}}+i \mathbf{k}_{2} \mathrm{r}_{\mathbf{b}}\right)\right|^{2} \\
& \sim \mid \exp \left[i 1 / 2\left(\mathbf{k}_{1}-\mathbf{k}_{2}\right)\left(\mathrm{r}_{\mathbf{a}}-\mathrm{r}_{\mathbf{b}}\right)\right] \exp \left[\mathrm{i} 1 /\left.2\left(\mathbf{k}_{1}-\mathbf{k}_{2}\right)\left(\mathrm{r}_{\mathbf{b}}-\mathrm{r}_{\mathbf{a}}\right)\right|^{2}\right. \\
& \sim \cos ^{2}\left[1 / 2\left(\mathbf{k}_{1}-\mathbf{k}_{2}\right)\left(\mathrm{r}_{\mathbf{a}}-\mathbf{r}_{\mathbf{b}}\right)\right],
\end{aligned}
$$


where $\exp \left(i k_{1} r_{a}\right)$ and $\exp \left(i k_{2} r_{b}\right)$ represent the plane waves associated with the two photons. As can be seen from the above relation the correlation function depends on the relative momentum of the two photons $q=1 / 2\left(k_{1}-k_{2}\right)$ and on the spatial seperation of the two sources $\Delta r=\left(r_{a}-r_{b}\right)$. Therefore by measuring $R$ it is possible to determine the spatial extent of the emitting sources.

The ideas of Hanbury Brown et al. [Han 56], are not limited to photons but can be applied also to other pairs of identical particles such as pions (bosons) [Bar 86a, Cha $91 \mathrm{a}]$ and nucleons (fermions)[Bau 92]. For instance, when two protons are emitted in close proximity in space and time, their wave function of relative motion can be modified by their mutual (coulomb) interaction and also by the quantum statistics of the two identical particles. The dependence of the space-time characteristics on the measurements of two-proton correlations can be understood by examining the theoretical expression for the two-proton correlation function that is given by [Gon 91a]

$$
\begin{aligned}
R(P, q) & =\frac{\Pi_{12}\left(p_{1}, p_{2}\right)}{\prod_{1}\left(p_{1}\right) \Pi_{2}\left(p_{2}\right)} \\
& =\frac{\int d^{4} x_{1} d^{4} x_{2} g\left(1 / 2 P, x_{1}\right) g\left(1 / 2 P, x_{2}\right)\left|\phi\left(r_{1}-r_{2}+P\left(t_{2}-t_{1}\right) / 2 m\right)\right|^{2}}{\int d^{4} x_{1} g\left(1 / 2 P, x_{1}\right) \int d^{4} x_{2} g\left(1 / 2 P, x_{2}\right)},
\end{aligned}
$$

where $\mathbf{P}$ and $\mathrm{q}$ are the total and relative momenta, $\mathrm{P}=\mathrm{p}_{1}+\mathrm{p}_{2}$ and $\mathrm{q}=1 / 2\left(\mathrm{p}_{1}-\mathrm{p}_{2}\right)$, respectively and $\phi$ is the relative wave function. The terms $x_{1}\left(r_{1}, t_{1}\right)$ and $x_{2}\left(r_{2}, t_{2}\right)$ are the space-time points of the emission of of protons 1 and 2 and $g(p, x)$ is the singleproton phase space emission function. Therefore measurements of the two proton correlations at small relative momenta can provide information about the space-time characteristics of the emitting system [Gou 91, Era 91, Gon 91, Bea 87, Kya 86, Che 87]. 
Proton correlation functions have been extensively studied as a function of the projectile and target mass [Ceb 89, Zhu 91] and the kinetic energy of the proton pairs [Boa 86, Poc 86]. As an example we present in Figure 1.3 (taken from Reference [Zhu 91]) a compilation of extracted source raciii plotted versus the average velocity $\mathrm{Vp}$ of the coincident proton pair for a variety of reactions; the average velocity $V p$ is normalized to the beam velocity $V_{\text {beam }}$. The size of the emitting source was determined by fitting the measured correlation function with the radius of the source treated as a free parameter. The source lifetime was assumed to be zero. The average velocity $V_{p}$ is given by $V p=1 / 2\left(p_{1}+p_{2}\right) / M_{p}$ where $p_{1}$ and $p_{2}$ are the momenta of the emitted frotons. For energetic protons $\left(V_{p} / V_{\text {beam }}>.5\right)$, the extracted source radius scales with the radius of the projectile and seems to be independent of the bombarding energy.

Finally, several groups have also examined two neutron correlations [Koo 89, Jak 91]. This variable has the advantage that there is no Coulomb interaction between the two neutrons or between the neutrons and the emitting source. However experimental difficulties arise from the relatively poor neutron detection efficiencies.

\section{d) Fragment - Fragment Correlations}

Fragment-fragment correlations can be used to examine the differences between sequential binary fission and true multifragmentation i.e. simultaneous multifragment breakup. In fact the possibility of discriminating between different multifragment mechanisms by means of fragment-fragment correlations has been studied by several groups [Lop 89, Ceb 90, Kim 92, Bou 89, Bau 93]. Selected examples of such studies are presented below.

The closer in time the fragments are emitted, the stronger their Coulomb interaction. This interaction influences the shape of the two-fragment correlation functions at small relative momenta. Therefore information about the time scale of 
fragment emission that may help distinguish between sequential and simultaneous decay can be obtained by means of the intensity interferometry technique that was described in the previous section. For example, Kim et al. [Kim 92] have applied the intensity interferometry technique to multifragment decay (fragment-fragment correlations) and have extracted time scales of the order $100-200 \mathrm{fm} / \mathrm{c}$ for the emission of $Z=4-6$ fragments in the $\mathrm{Ar}+\mathrm{Au}$ reaction at $35 \mathrm{MeV} / \mathrm{A}$. Such an emission time is compatible with the picture of interacting sequential decays.

In another study, Lopez et al. [Lop 89] have utilized the momentum tensor to examine the shape of multifragmentation events in momentum space. The momentum tensor is given by:

$$
Q_{i j}=\sum_{n=0}^{N} \gamma p_{i}^{(n)} p_{j}^{(n)}, i, j=\{x, y, z\}
$$

where the sum runs over the number $N$ of fragments of the event, and $p_{1}^{(n)}$ is the momentum coordinate in the direction $i$ of fragment $n$ in the center-of-mass of the event. The quantity $\gamma$ is a scalar weighting factor which can be used to emphasize some parts of the momentum space. In their analysis [Lop 89] events arising from sequential decay exhibit an elongated shape, whereas events associated with a simultaneous breakup lead to a spherical shape. Several experimentalists [Ceb 90, Hag 89] have applied this approach to multifragment events produced in heavy ion reactions. For instance Cebra et al. [Ceb 90] found that in the 35-85 MeV/A Ar + V reactions multifragment events exhibited an elongated shape at the lowest bombarding energies thus demonstrating that the fragments were emitted sequentially. At higher energies however more spherical shapes were observed that could be associated with events produced between the sequential and simultaneous limits. 
Finally Bauge et al. [Bau 93] have measured two-fragment reduced-velocity correlation functions for the symmetric $\mathrm{Kr}+\mathrm{Nb}$ system over a broad range of bombarding energies $(E / A=35,45,55,65$, and $75 \mathrm{MeV})$. The two-fragment reducedvelocity was determined by $V_{\text {red }}=V_{\text {rel }} /\left(Z_{1}+Z_{2}\right)^{1 / 2}$ where $V_{\text {rel is }}$ is the velocity between two detected fragments whose charges are $Z_{1}$ and $Z_{2}$. The measured correlation functions demoustrated that the mean fragment emission lifetimes $\tau$ initially decreased with increasing beam energy. However, for beam energies greater than $E / A=55 \mathrm{MeV}$, $\tau$ was observed to saturate at $\tau \sim 125 \mathrm{fm} / \mathrm{c}$. The authors concluded that such a saturation could result from the onset of simultaneous multifragmentation at a E/A $>55 \mathrm{MeV}$. It is noteworthy that the observed $\tau$ values for $\mathrm{E} / \mathrm{A}>55 \mathrm{MeV}$ are consistent with those ( $\tau$ $\sim 100 \mathrm{fm} / \mathrm{c}$ ) predicted for multifragment disintegration resulting from bulk instabilities of nuclear matter at low density [Ber 83, Boa 88 , Gro 90, Bau 92a, see also Chapter 2].

\section{e) Population Ratios}

Consider a system $\mathrm{W}$ in thermal equilibrium at temperature $\mathrm{T}$ and a subsystem $\mathrm{w}$ for which it is justified to apply the Boltzmann statistics. Then the ratio of the probabilities for obscrving $w$ in two different states $w_{1}$ and $w_{2}$ is given by

$$
R=\frac{P_{1}}{P_{2}} \rightarrow R \sim \exp \left[\frac{E_{1}-E_{2}}{T}\right]=\exp \left[-\frac{\Delta E}{T}\right],
$$

where $E_{1}$ and $E_{2}$ are the corresponding internal energies for states $w_{1}$ and $w_{2}$.

Equation 1.6 can be applied to nuclear systems by considering $\mathrm{W}$ to be a nucleus and $w$ a cluster evaporated from $W$. The objective is then to determine the nuclear temperature $T$ by measuring the ratio of populations $R$ where $\Delta E$ is a known quantity representing the energy seperation between the two states $w_{1}$ and $w_{2}$ of the emitted cluster. If the emitted cluster $w$ is a primary fragment whose states $w_{1}$ and $w_{2}$ are 
associated with spins $s_{1}$ and $s_{2}$ respectively, then a more complete expression for the primary population ratio is given by

$$
R=\frac{\left(2 s_{2}+1\right)}{\left(2 s_{1}+1\right)} \exp \left[-\frac{\Delta E}{T}\right]
$$

The main limitation of this method is the a priori assumption that the emitting system is in thermal equilibrium. Furthermore the method may be correct only if the studied clusters have kept the memory of the initial thermal bath W, i.e. if the observed decay is a primary decay. By including clusters in the analysis resulting from a "side feeding" i.e. from a decay other than the primary decay, this method leads to a wrong temperature value.

This approach has been applied to particle emission from both bound [Mor 84] and unbound states [Poc 87, Nay 92, Kun 91]. In the former case the populations can be determined by detecting the clusters and/or the gamma rays associated with the decay of these clusters. For example, by using the $\gamma$-ray emission technique, populations of $\mathrm{Be}$ and $\mathrm{B}$ nuclei emitted in their ground and excited states from the reaction ${ }^{14} \mathrm{~N}+{ }^{12} \mathrm{C}$ were measured [Mor 84]. The extracted temperatures were found to be consistent with emission from a thermally equilibrated compound nucleus for bombarding energies below $112 \mathrm{MeV}$. At higher bombarding energies the population distributions remained constant.

The side feeding problem mentioned earlier is responsible for an underestimate of the deduced temperatures, but its effect decreases when the difference $\Delta E$ increases; the extracted values are more reliable when the two states $w_{1}$ and $w_{2}$ are wide apart. This effect can be seen clearly in Figure 1.4 (taken from [Poc 87]) which shows the apparent temperatures extracted from the relative populations of particle unbound states for various pairs of excited clusters formed in the $60 \mathrm{MeV} / \mathrm{A} \mathrm{Ar}+\mathrm{Au}$ reactions. The 
extracted temperatures are larger for states that are energetically wide apart. Therefore since the $\gamma$-ray method is restricted to low-lying bound states it should be more susceptible to side feeding then the particle emission method.

Although the particle emission method may be less sensitive to side feeding effects, the extracted temperatures are substantially lower than temperatures determined with other methods. For instance the mean emission temperature of the sources formed in the $60 \mathrm{MeV} / \mathrm{A} \mathrm{Ar}+\mathrm{Au}$ reactions was determined to be around $5.5 \mathrm{MeV}$, which is about a factor of 3 lower than the temperature inferred from the slope of kinetic energy spectra. Similar emission temperatures (around $5 \mathrm{MeV}$ ) have been inferred with the particle emission method for several systems over a wide bombarding energy range [ Nay 92, Bor 92, Che 88]. Even at $200 \mathrm{MeV} / \mathrm{A}$ [Kun 91], which should be well in the fireball regime, emission temperatures of only $6 \mathrm{MeV}$ are measured. Thus it appears that the temperature extracted from the relative population of states with the particle emission method is weakly dependent on the incident beam energy.

\section{f) Light Particle Multiplicity}

Early attempts to identify the origin of multifragmentation relied mainly on information arising from the experimentally determined inclusive fragment distributions. For instance the observation [Fin 82], nearly a decade ago, that the fragment distribution measured in high-energy proton-induced reactions followed a power law $\left(\sim A^{-\tau}\right)$ was taken as an indication of the existence of a liquid-gas phase transition in finite nuclei. It has, however, been demonstrated that the observed power law dependence is insufficient to identify conclusively the process of multifragmentation, since these distributions can be reproduced by different models (i.e. percolation, nuclear shattering... [See Chapter 2]). A definite identification of this process may be possible, if along with complex fragments, quantities such as light 
charged particle and neutron multiplicities are measured. Such quantities may serve as a coarse measure of the impact parameter as well as a measure of the excitation energy.

Several groups have measured light particle multiplicities in nearly $4-\pi$ detectors [Mor 88, Jia 88, Cha 91, Jac 91, Pi 91, DeS 91 Sch 91, Bo 92 ] and below we present selected examples of such measurements.

By utilizing a $4 \pi$ Gd-loaded liquid scintillator detector Piasecki et al [Pia 91] have measured the multiplicity of neutrons associated with the $29 \mathrm{MeV} / \mathrm{A} \mathrm{Pb}+\mathrm{Au}$ collisions. The measurements demonstrated the presence of strong correlations between impact parameter and neutron multiplicity. Massive fragments were unlikely to survive the most dissipative, central collisions, selected by high neutron multiplicity gates. In such events, approximately one-third of the neutrons were released and the fragment yields were found to decrease in an exponential fashion with increasing mass.

In a different experiment, Jiang et. al. [Jia 88] measured the emission of neutrons in the energy range 27-77 MeV/A for the systems $\mathrm{Ar}+\mathrm{Au}$, Th. In this work the average neutron multiplicity was found to increase initially as a function of energy, reached a saturation value around $40 \mathrm{MeV} / \mathrm{A}$ and remained approximately constant with increasing bombarding energy. This behavior indicated the occurrence of a saturation of the thermal energy deposited in the system(s). As the bombarding energy increased preequilibrium processes carried away a larger fraction of the available energy.

In another study, the authors of [Bal 91, Lot 91] have measured both neutrons and charged products in the ${ }^{209} \mathrm{Bi}+{ }^{136} \mathrm{Xe}$ reaction at $29.2 \mathrm{MeV} / \mathrm{A}$ using a combination of two nearly $4 \pi$ detectors. They found binary collision dynamics to dominate for all degrees of dissipation, as determined by the multiplicities of the neutrons and charged particles. Even the requirement of multiple intermediate mass fragments did not select a different reaction mode. They concluded that either the IMFs are emitted from a dinuclear system even in central collisions or that central collisions are not sufficiently 
isolated by the requirement of high multiplicities. This result may represent a strong constraint for models aiming at explaining the origin of IMFs in intermediate-energy heavy ion reactions.

Finally, Bowman et al. [Bo 92] detected charged complex fragments in the 50 $\mathrm{MeV} / \mathrm{A}{ }^{129} \mathrm{Xe}+{ }^{12} \mathrm{C},{ }^{27} \mathrm{Al}$, nar $\mathrm{Cu}$, and ${ }^{197} \mathrm{Au}$ reactions along with light charged particles using a low threshold $4 \pi$ detector. They observed that the fragment multiplicity distributions and charge distributions were strongly dependent upon the charged particle multiplicity and showed a large degree of target independence. They concluded that the fragment multiplicity was determined by the excitation energy deposited in the system and that statistical concepts may be useful in calculating the branching ratios for fragment emission. Furthermore the measured distributions were compared with hybrid model calculations which incorporate dynamical and statistical aspects (BUU + EES model, it is discussed in Chapter 2) and showed reasonable agreement.

Despite the intense experimental efforts presented above, multifragmentation still remains elusive in its interpretation. It is still not clear whether multifragmentation can be characterized as a homogeneous or heterogeneous process. Furthermore the aspect of sequentiality still remains uncertain. Although some experimental progress [Rou 93] has been made, by isolating and characterizing what appear to be true multifragmentation sources a precise assignment of excitation energies, angular momentum... is still missing. Further experiments using $4 \pi$ detectors along with improved model calculations are needed if one is to understand the "complex" process of multifragmentation.

\subsection{Goal of Project}


At low energies it has been well established that complex fragments can be produced in deep inelastic and compound nucleus reactions. The concept of the compound nucleus has also been successfully applied to the $10-30 \mathrm{MeV} / \mathrm{A}$ bombarding energy regime. At intermediate $(30<\mathrm{E} / \mathrm{A}<100)$ bombarding energies, however, an increasing yield of 3 -body and higher-order events is observed that raises several questions. What is the origin of the multibody decays observed in these reactions? Is the observed multifragment decay due to a prompt nuclear disintegration, or instead to a compound nucleus undergoing a series of binary decays? Is this decay controlled by dynamics or statistics? The goal of this thesis was to search for the conditions under which multifragmentation becomes a dominant decay channel and to investigate questions such as the ones raised above. To this end we studied the systems $\mathrm{Au}+\mathrm{C}$, $\mathrm{Al}, \mathrm{V}, \mathrm{Cu}, \mathrm{Au}$ at $60 \mathrm{MeV} / \mathrm{A}$.

Complex fragment emission at intermediate energies has been studied by others and the above reactions provide a natural extension of the reaction systematics established in those studies[Rou 93]. However, there were some special features of this experiment that made it unique. For instance, the projectile used in the experiment presented in this thesis, was much heavier than the projectiles utilized in the studies referenced above. Such a heavy nucleus should bring large amounts of mass, angular momentum and excitation energy into the center-of-mass of the colliding nuclei and thus make new phenomena experimentally accessible.

Furthermore, the choice of targets provided a variety of target-projectile combinations ranging from very asymmetric to symmetric collisions, in which the excitation energy per nucleon available in the center-of-mass system increases from $\mathrm{E}^{*} / \mathrm{A}=3.2 \mathrm{MeV}\left(\mathrm{E}_{\mathrm{cm} \mathrm{C}}=680 \mathrm{MeV}\right)$ to $\mathrm{E}^{*} / \mathrm{A}=15 \mathrm{MeV}\left(\mathrm{E}_{\mathrm{cm} \mathrm{Au}}=5910 \mathrm{MeV}\right)$. The transition between statistical binary decay of an incomplete fusion source and 
multifragmentation should be reached in these reactions, since the onset of multifragmentation is generally predicted to occur in the range of about $\mathrm{E}^{*} / \mathrm{A} \sim 4 \mathrm{MeV}$ [Gr 85, Bon 85].

Finally, it is interesting to know the excitation energy of the hot intermediate nuclear systems formed in these reactions. In the work of Roussel et al. [Rou 93] the excitation energy $E^{*}$ was determined from the measured source velocity within the picture of the incomplete fusion model. However, it is desirable to determine the excitation energy $E^{*}$ of the source independent of any assumptions made about the decaying system.

One way of determining $E^{*}$, is to measure the neutron multiplicity associated with these hot systems, since neutron emission is expected to carry away a large part of the excitation energy. Furthermore neutrons will be emitted whatever the decay process is. For instance, in the low energy domain, the emission of neutrons is understood in terms of dominantly sequential evaporation from massive fragments and a relatively weak contribution from nonequilibrium processes. On the other hand, at higher bombarding energies the emission pattern is expected to reflect the increased role of faster processes such as nonequilibrium emission and evaporation during dissipation and equilibration. In addition, the multiplicity of evaporated light particles has already proven to be a reliable measure for energy dissipation in nuclei [Gal 88, Olm 87 a, Tsa 89 , Jia 89 , Sch 91]. In this experiment we have attempted to measure the neutron multiplicity $M_{n}$, by utilizing a novel neutron calorimetric approach [ $\mathrm{Pa} 88, \mathrm{~Pa} 90]$ suitable for reverse kinematic reactions. 


\section{Chapter 2}

\section{Theory}

Several theories have been developed in order to explain the multifragment production observed in intermediate energy heavy ion reactions. These theories can be classified as either statistical or dynamical.

Statistical theories assume a decoupling of the interaction and decay processes. They begin at the dissasembly stage where the intermediate source of fragments, formed either in a complete or incomplete fusion process, is assumed to attain equilibrium prior to the emission process. These theories take minimal account of entrance channel effects and cannot reproduce the non-equilibrium features observed at intermediate energies. On the other hand, dynamical models take into account the entire evolution of the reaction from its early stages to the breakup stage. Although these theories may provide a good description of the preequilibrium emission of light particles observed at intermediate energies, they cannot accurately describe the statistical decay of the system in the long time limit.

In this chapter a brief description is given of some of the main multifragmentation theories. Initially the statistical theory of complex fragment emission from compound nucleus is discussed, since models inherited from low-energy nuclear reactions may still 
be valid at intermediate energies and provide a useful guide for studying multifragmentation. Subsequently statistical and dynamical models that predict multifragmentation are presented along with selected calculations for some of the models.

\subsection{Origin of Complex Fragments in Compound Nucleus Reactions}

Light particle evaporation and fission are two modes of decay of a compound nucleus. They can be regarded as the limiting forms of a single statistical process, whose underlying connection is provided by the mass asymmetry coordinate [Mo 75]. Light-particle emission occurs at extreme asymmetries, while fission occurs at symmetry. Complex fragment emission occurs at intermediate mass asymmetries, thus providing a natural link between the two limiting processes.

Each complex fragment is emitted with a probability that depends upon the barrier associated with that particular mass asymmetry. This barrier is the energy, with respect to to the corresponding sphere, of the saddle-point shape [Coh 74] restricted to a fixed mass asymmetry and is called the "conditional saddle point" [Mor 75]. The locus of all conditional saddle points along the mass asymmetry coordinate defines a ridge line in the potential-energy surface of the nucleus. The general shape of the ridge line depends on whether the fissility parameter $x$ [Coh 63, Coh 74] lies above or below the BusinaroGallone point [ $\mathrm{Bu} 55]$. This is the point where the second derivative of the potential energy with respect to the mass-asymmetry coordinate evaluated at symmetry goes to zero. In the liquid drop model [Ni 65] such a transition occurs at $\mathrm{x}_{\mathrm{BG}}=0.396$ for zero angular momentum. The shape of the ridge line for systems above and below the Businaro-Gallone point is shown in Figure 2.1 by the solia ines. Light compound nuclei (see Figure $2.1 \mathrm{~b}$ ) have fissility values below $\mathrm{x}_{\mathrm{BG}}$, and the ridge line shows a 
maximum at symmetry. On the other hand, for heavy nuclei (see Figure 2.1a) above the Businaro-Gallone point the ridge line presents minima at symmetry and extreme asymmetries.

When a compound nucleus is confronted with a potential-energy ridge, it can select any asymmetry through which to decay. As shown in Figure 2.2 a continuous set of trajectories can be imagined that originate in the compound nucleus region, reach up to the ridge line, and then descend towards the product region. For most compound nuclei, the shape at the ridge line is approximately the shape at the scission line; while for the heavier systems near symmetry there is some difference between the saddle and scission lines, for the lighter systems at all mass asymmetries and for the heavier systems at large asymmetries, the saddle and scission configurations are almost degenerate. Therefore as the compound system approaches the ridge line it is committed to decay with the corresponding saddle asymmetry, and the probability of overcoming the ridge at the various asymmetries can be translated into mass distributions. The relationship between the yield $Y$ and the conditional barrier $B(z)$ for a given complex fragment is given approximately by :

$$
\mathrm{Y} \propto \exp \left(\frac{-\mathrm{B}(\mathrm{z})}{\mathrm{T}_{\mathrm{Z}}}\right)
$$

where $T_{Z}$ is the saddle temperature at the asymmetry $\mathrm{Z}$. Details on how this expression is obtained are given in Appendix 1.

From relation 2.1 we can deduce that the favored decay channels are those associated with the lowest points of the ridge line. A complex fragment has a low probability for emission when the conditional barrier is high and vice versa. This emission characteristic is shown qualitatively by the dashed lines in Figure 2.1. The yields of complex fragments (dashed lines) are a reflection of the conditional saddle 
points (solid lines). A heavy compound nucleus $\left(\mathrm{X}_{\mathrm{BG}}<0.396\right)$ displays sort of a $\mathrm{W}$ shaped yield curve (see Fig. 2.1a) with high yields at symmetry (fission peak) and extreme asymmetries (light particle evaporation), while a light nucleus ( $\left.X_{B G}>0.396\right)$ exhibits a U-shaped yield curve (see Fig. 2.1b) with a minimum at symmetry.

The theory described above has been tested in several experiments [Ch $88 \mathrm{a}$, Ch $88 \mathrm{~b}$, Ch 90, De 91]. As an example, we show in Figure 2.3 the experimental cross-sections from the ${ }^{63} \mathrm{Cu}+{ }^{12} \mathrm{C}$ reactions $[\mathrm{De} 91]$ at several bombarding energies. As expected, the charge distributions show the characteristic U-shape associated with the decay of a compound system below the Businaro-Gallone point. Furthermore, a change in the absolute cross-section as well as an evolution in the shape of the charge distributions can be seen in Figure 2.3. The observed flattening of the charge distribution with increasing energy can be explained by the increase in temperature of the system which tends to make all of the decay channels more equally probable. As predicted by equation 2.1 when $\mathrm{T} \rightarrow \infty \Rightarrow \mathrm{Y} \rightarrow>$ constant. The solid lines represent predictions with the statistical code GEMINI which is based on the above formalism. GEMINI is described in some detail in Appendix 2.

From the above we see that in low- and moderately high-energy reactions complex fragments are produced from the binary decay of a compound nucleus. At higher excitation energies however, the primary binary decay products may be also very excited, and have a significant probability of decaying in turn into two additional fragment and so on. In this way multifragmentation can be considered a natural extension of the processes occurring at low energies. This case is examined in the following section.

\subsection{Multifragmentation models.}




\subsubsection{Statistical Theories.}

\section{a) Sequential Binary Decay}

One of the possible ways of generating several fragments is through a sequence of binary decays [Mor 87, Mor 88] of which the initial binary division of the reacting system can be either direct or statistical. If the excitation energy of the system is large enough, each of the primary fragments producied in the initial binary decay can contain large amounts of energy and decay further by undergoing a compound binary division. For progressively higher excitation energies the decay chains should become increasingly long and complex.

Direct binary followed by compound binary decay is a process well established at intermediate energies [Olm 87, Bor 90, Tok 90]. For example a two step decay chain has been observed in the reaction $\mathrm{Mo}+\mathrm{Mo}$ at $\mathrm{E} / \mathrm{A}=18.7$ and $23.7 \mathrm{MeV}$ [Olm 87], where a deep inelastic reaction is followed by the binary compound decay of one or both primary fragments. Furthermore incomplete fusion followed by fission can be also approximately characterized as a direct binary plus compound binary decay. In this case the resulting incomplete fusion product(s) relaxes to a compound nucleus which proceeds to decay statistically; one or more complex fragments may be observed in the exit channel [Cha 90, Mor 88, Rou 93].

Finally, multifragmentation can result from the successive statistical binary divisions of a complete fusion product i.e. a compound nucleus. This process of sequential-binary decays, controlled at each step by the compound nucleus branching ratios is known as nuclear comminution [Mor 88 , Mor 87] and may be responsible for a substantial background to other predicted multifragmentation mechanisms as mentioned earlier. 
However, at the high bombarding energies needed to produce hot nuclei, nuclear comminution may be limited by the ability of the system to form a compound nucleus. Furthermore, should two sequential binary decays occur too close in space-time, they would interact to an extent incompatible with the definition of sequentiality. If this occurs, it may be more suitable to consider alternative models in which the fragments are emitted simultaneously; in these models the fragments are formed together in a certain volume, and are in chemical and physical equilibrium with each other, although it is not clear how a system may have achieved such an equilibrium.

\section{b) Chemical equilibrium theories.}

\section{Liquid-Vapor Equilibrium}

The nucleon-nucleon interaction is attractive at short distances and repulsive at very short distances. This behavior is qualitatively similar to the molecular interaction of substances which can exist in liquid and gaseous phases, a simple example being the Van der Waals fluid. Based on such considerations it is possible that hot nuclear matter may be able to undergo a liquid-gas phase transition, thus producing nuclear droplets which are the observed heavy fragments (multifragmentation) [Sau 76, Ber 83, Si 83, Lop 84]. In fact, Fermi-Thomas[Bar 80, Bar 81, Bra 84] and Hartree-Fock [Sau 76, Lam 81] calculations for nuclear matter lead to isotherms (see Figure 2.4) that are quite similar to those of the Van der Waals equation. Figure 2.4, taken from Reference [Sau 76], is an example of predicted isotherms for nuclear matter.

The probability $\mathrm{M}_{\mathrm{j}}$ of finding a cluster of $\mathrm{j}$ particles in a fluid is given by [Mor 93 , Ban 39, Fre 39]:

$$
M_{j}=\exp \left(\frac{-c j 2 / 3}{T}\right) \exp \left(\frac{j\left(\mu-\mu_{L}\right)}{T}\right) M_{0} j^{-\tau} \quad
$$


where $\mu$ is the chemical potential of clusters of size one, $\mu_{L}$ is the chemical potential of the liquid, $\mathrm{T}$ is the temperature, $\mathrm{c}$ is the surface energy coefficient, and $\tau$ is a critical exponent. The first factor in the above equation is related to the surface energy and the second factor is associated with the volume energy. The factor $j^{-\tau}$ arises from the energy independent statistical weight of a cluster of size $j$ and becomes important at the critical temperature $T_{c}$ where the first two exponential factors are approximately equal to 1 .

When $\mu_{L}>\mu$ the system is in the gas phase, and the cluster probability falls exponentially with $\mathrm{j}$. On the other hand, when $\mu_{\mathrm{L}}<\mu$ the system is in the liquid phase . Finally, when $\mu_{L}=\mu$ the liquid and gas phases are in equilibrium.

At the critical temperature, which is the highest temperature at which the two phases can exist in equilibrium, the densities of the liquid and gas are equal and $c=0$. At this point the fragment size distribution is determined by the last factor of equation 2.2 and the cluster distribution assumes a power law form:

$$
M_{j} \propto j^{-\tau} \quad
$$

It is this power-law distribution that many authors claim to have identified in a variety of inclusive experiments. However, several investigations have shown that the power law dependence does not provide a unique signature for a liquid-gas phase transition near the critical point. Fragment distributions with a power-law dependence can be reproduced also with statistical equilibrium models utilizing canonical/microcanonical approximations [Gro 82, Gro 83, Bon 83, Bon 85, Ran 81] and percolation models [Ca 88, Bau 85, Bau 86].

\section{Canonical/Microcanonical Approximations}


A problem with the liquid-vapor equilibrium theory is that it neglects the Coulomb interaction, the nuclear masses, and the level densities of the nuclei. Other chemical equilibrium models [Gro 82, Gro 85 , Bon 83 , Bon 85 , Ran 81 ] however, have been proposed that include the above factors. In these models the fragments are treated as spherical entities that are randomly located within a somewhat arbitrarily chosen "freeze-out" volume. Furthermore, the fragments are not allowed to be closer to each other than a minimum distance that is also arbitrarily chosen. Statistical mechanics is used to describe the nuclear system at the "freeze-out" volume by sampling the relevant phase space over all possible fragmentations. In an exact treatment this must be done in a microcanonical ensemble [Gro 82, Gro 85] for fixed values of the total energy, mass, momentum, etc. For large systems the problem has been simplified by employing a canonical ensemble in which the mean energy is fixed or a grand canonical ensemble in which the mean number of particles and the mean energy are fixed [Bon 83, Bon 85].

As an example, Figure 2.5 illustrates the results of the microcanonical calculation by Gross et al. [Gro 87] for ${ }^{238} \mathrm{U}$. Four decay mechanisms can be identified with the relative yield of each decay channel depending on the excitation energy E*. For low $\mathrm{E}^{*}\left(\mathrm{E}^{*}<100 \mathrm{MeV}\right)$ values, the evaporation of light particles is the dominant exit channel. For $\mathrm{E}^{*}>100 \mathrm{MeV}$, however, fission becomes more likely. Multifragmentation, referred to in Figure 2.5 as cracking, takes place at a higher excitation energy with a maximal yield at about $\mathrm{E}^{*} / \mathrm{A} \sim 4 \mathrm{MeV} / \mathrm{A}$. At $7 \mathrm{MeV} / \mathrm{A}$ the onset of vaporization occurs.

A major drawback in this group of models is the arbitrary choice of the size $\mathrm{R}$ of the freeze-out volume within which the equilibrium is calculated because the results may depend substantially on this choice. For instance the Coulomb energy of the fragments that are located within the "freeze-out" volume will change tremendously depending on the choice for $R$. 


\section{c) Percolation Theories}

Percolation approaches[Bau 85, Bau 86, Des 87a, Des 87b, Cam 86, Cam 88, Ngo 90] have also been used to describe the fragmentation of nuclei. These theories treat the nucleus as a three dimensional lattice of nucleons connected by bonds, where the probability $\mathrm{p}$ for breaking a bond depends on the excitation energy of the nucleus. By increasing the excitation energy more bonds break, and the system evolves from one large cluster to many small clusters of nucleons.

For an infinite system, percolation theory predicts that above a certain critical value $p_{c} \quad\left(p>p_{c}\right)$ the system breaks up into many small clusters, while for $p<p_{c}$ one infinite cluster is present along with smaller clusters. The similarity of this result with the behavior of systems exhibiting 2 nd order phase transitions, like the Van der Waals gas, has led to the use of percolation models to simulate such transitions for finite systems. By relating the heaviest fragment produced in a finite system (such as in a nuclear reaction) with the infinite cluster and the smaller fragments (in the finite system) with the smaller clusters (in the infinite system) this theory can be used to describe the process of multifragmentation. It is interesting to note that percolation theory predicts an inclusive mass distribution following a power law.

\section{d) Statistical Shattering Theories.}

Several models[Boh 83, Huf 83, Aic 84] describe multifragmentation as a nonthermal disassembly process, i.e. they assume that two colliding nuclei can break up on impact and shatter like a piece of brittle material(e.g. glass...). As a representative example of this class of models we will describe below the essential features of the model developed by Aichelin and Hufner[Aic 84]. 
Aichelin and Hufner consider that all possible fragmentations occur with equal probability. If $P(m, Z)$ is the probability of producing a fragment of charge $Z$ and multiplicity $m$ from an initial nucleus with charge $Z_{0}$, then normalization of the probability $P(m, Z)$ to 1 and conservation of charge lead to the following constraints :

$$
\begin{gathered}
\sum_{m} P(m, Z)=1 \\
\sum_{m} \sum_{Z=1}^{Z_{0}} m Z P(m, Z)=Z_{0} .
\end{gathered}
$$

$P(m, Z)$ can then be calculated from the requirement of minimal information under the constraints given by equations 2.4 and 2.5 . The constraints are introduced with the technique of Lagrange multipliers. By summing $\mathrm{P}(\mathrm{m}, \mathrm{Z})$ over $\mathrm{m}$, the resulting probability distribution is (approximately) :

$$
P(Z) \approx\left[\exp \left(\frac{1.28 Z}{Z_{0}^{1 / 2}-1}\right)\right]^{-1}
$$

Equation 2.6 demonstrates that the shape of the charge yield curve is completely determined by charge conservation. Furthermore it has been demonstrated in reference [Aic 84] that the no-free-parameter equation 2.6 reproduces well the shape of experimentally determined charge distributions, at least as well as a fit using the form $\mathrm{d} \sigma / \mathrm{dZ} \sim \mathrm{A}^{-\tau}$ with the adjustable parameter $\tau$.

The approach described above, however, has some drawbacks, such as its lack of energy dependence and its inability to relate the mass distributions to other observables. A possible way to introduce an energy dependence in this problem has been undertaken by Moretto et al. [Mor 86, Mor 93]. Moretto et al. evaluate the mass (or charge) 
distribution with the additional constraint of a fixcd amount of generated surface. Their approach is based on the fact that it takes energy to produce the extra surface associated with fragment formation. The additional constraint used in their calculations is

$$
\sum_{m} \sum_{Z=1}^{Z_{0}} k m Z^{2 / 3} P(m, Z)=S
$$

where $\mathrm{S}$ is the generated surface. The resulting $\mathrm{Z}$ distribution is then given by:

$$
\mathrm{d} \sigma / \mathrm{dZ}=\frac{1}{\exp \left(\mathrm{DZ}+\mathrm{AZ} \mathrm{Z}^{2 / 3}\right)-1}
$$

where $\mathrm{D}$ and $\mathrm{A}$ are constants. It should be noted that the above equation is very similar to the equation for the droplet size in liquid-vapor equilibrium(see section 2.2).

\subsubsection{Dynamical Theories}

The dynamical descriptions of energetic heavy ion collisions are generally based on one-body transport models. At low energies (just above the Coulomb barrier) where mean field dynamics is important, Time-Dependent Hartree-Fock (TDHF) calculations [Dav 78, Neg 82] describe phenomena like fusion or deep inelastic scattering with

relative success. At higher energies, however, these calculations predict too much transparency, and the two colliding nuclei can slip through each other and survive intact. This occurs because two- body collisions, which become important due to the short-range hard-core repulsion are not included in the TDHF equation. Attempts [Be 88, Sch 89 , Aic 91, Gr 87, Bo 89, Bo 90a, Bo 90b] to incorporate two-body collisions have been performed by augmenting the Vlasov equation by a Pauli blocked collision 
term $I_{\text {coll }}$ of the Boltzmann form; the classical analog of the TDHF is the Vlasov equation. The resulting Boltzmann-Nordheim-Vlasov (BNV) equation (also referred to as BUU for Boltzmann-Uehling-Uhlenbeck equation, VUU for Vlasov-UehlingUhlenbeck and LV for Landau-Vlasov equation) is given by :

$$
\frac{\partial \mathrm{f}}{\partial \mathrm{t}}+\frac{\mathrm{p}}{\mathrm{m}} \nabla_{\mathrm{r}} \mathrm{f}-\nabla_{\mathrm{r}} \mathrm{U} \nabla_{\mathrm{p}} \mathrm{f}=\mathrm{I}_{\text {coll }}
$$

where $\mathrm{U}$ is the mean field potential and $\mathrm{f}$ is the single-particle distribution function in phase space. The collision is simulated by solving the BNV equation and will be discussed in some detail in Chapter 6.

In this theory multifragmentation may occur as a result of regions of instability that the system encounters in its dynamical evolution. One class of instabilities that may play an important role in multifragmentation are surface instabilities of the Rayleigh type [Mo 92]. As an example, Figure 2.6 shows calculations utilizing the BNV equation for ${ }^{92} \mathrm{Mo}+{ }^{92} \mathrm{Mo}$ collisions at $55 \mathrm{MeV} / \mathrm{A}$ and $\mathrm{b}=0 \mathrm{fm}$ for different time steps; initially a disk develops, due to the side-squeezing of nuclear matter, which then breaks up into several fragments due to surface instabilities. In this case, the system escapes from the high surface energy of the disk by breaking up into a number of spherical fragments with less overall surface. Besides disks, other shapes for hot nuclei have been observed in BNV calculations [Bau 92a, Bor 92, Xu 93] that may be also subject to surface instabilities. These shapes include toroids and bubbles.

Since nuclear matter behaves qualitatively in the same way as a Van der Waals fluid another type of instability that can be considered as a possible initiator of multifragmentation is the spinodal instability. This instability develops when the system enters the spinodal region. This region shown in Figure 2.4 by the dashed part of the pressure curves represents an area of dynamic and thermodynamic instability 
and is associated with a negative incompressibility modulus $\mathrm{K}=\rho(\partial \mathrm{P} / \partial \rho)$. In this region a single homogeneous phase is unstable, and the system breaks up into a liquid phase, and a gas phase [Ber 83, Lo 84, Pet 87, Hei 88]; this occurs by the formation of liquid droplets embedded in saturated vapor. Since the spinodal instability can occur in an infinite system, it is also known as volume or bulk instability.

The strength of dynamical theories is their excellent description of the interaction between two colliding heavy nuclei during the first stages of the interaction. However, they have some serious drawbacks. The Pauli principle, for example, is not strictly respected. Furthermore, these calculations cannot correctly represent the statistical decay of hot nuclei at the late stages of the reaction, because they do not include fluctuations. In References [ $\mathrm{Sn} 88, \mathrm{Co} 91$ ] this last difficulty has been circumvented by combining a dynamical model that simulates the pre-equilibrium phase of the reaction (early stages of the reaction) with a statistical model that simulates the decay of the excited system during the final stages. Such calculations may be a promising avenue towards understanding multifragmentation. 


\section{Chapter 3}

\section{Experimental}

The experimental data, from the $60 \mathrm{MeV} / \mathrm{A}{ }^{197} \mathrm{Au}+{ }^{12} \mathrm{C},{ }^{27} \mathrm{Al},{ }^{51} \mathrm{~V},{ }^{63} \mathrm{Cu}$, and ${ }^{197} \mathrm{Au}$ reactions, presented in this thesis were collected over a 120-hour running period (during October of 1991) at the Bevalac accelerator complex of the Lawrence Berkeley Laboratory. The following sections give a detailed description of the techniques and apparatus used to collect the experimental data .

\subsection{Reverse Kinematics}

The $60 \mathrm{MeV}{ }^{197} \mathrm{Au}$-induced reactions were studied by using the reverse kinematics technique. In this technique the heavier nucleus is used as the projectile and the lighter nucleus as the target. The kinematics of this process are quite different from those in normal kinematics (in which the lighter partner is used as the projectile) and will be explained below by means of an example.

Figure 3.1 is a kinematics diagram in the $V_{\perp}-V_{\|}$plane, illustrating the emission of a given fragment from a compound nucleus in reverse kinematics. The vector $V_{\delta}$ 
represents, in the laboratory frame, the velocity of a source formed in a complete fusion process (compound nucleus). Because of the large asymmetry of the entrance channel the source velocity is quite large and can be slightly less than the beam velocity. For example in the case of the $60 \mathrm{MeV} \mathrm{Au}+\mathrm{C}$ reactions the velocity of the source resulting from complete finsion between the projectile and the target is $95 \%$ of the beam velocity. The binary decay of this source results in the emission of complex fragments whose velocities in the center of mass are determined mostly by the Coulomb repulsion between the two decay products. In Figure $3.1, \mathrm{~V}_{\mathrm{c}}$ represents the velocity of a particular complex fragment emitted in the center-of-mass (c.m.) of the source. The locus of all the emission velocities for that particular fragment (i.e. the emission of the fragment at all angles) in the $V_{\perp}-V_{\|}$plane is represented by the circle.

The emission velocities of the complementary fragment emitted in the binary decay can be also represented by a circle (not shown in Figure 3.1). The radii of these circles depend upon the Coulomb energy available in the binary decay process and the mass asymmetry of the decay products. Because conservation of momentum requires that the smaller fragment have a larger velocity in the source frame, the radius of the circles will decrease monotonically with increasing fragment charge.

Let us consider now a given angle $\theta$ in the lab for which the circle can be intersected at two points as shown in Figure 3.1. The two intersection points represented in the lab frame by the velocity vectors $\mathbf{V}_{\mathbf{a}}$ and $\mathbf{V}_{\mathbf{b}}$ (see Figure 3.1) can be associated with the vector velocities $\mathrm{Ve}$ of a fragment emitted either forward or backward in the center of mass, respectively. The interesting feature here is that $V_{a}$ and $\mathrm{V}_{\mathrm{b}}$ have the same direction thus allowing us, by setting the detector at a given laboratory angle $\theta$, to observe both the high velocity $\left(\mathrm{V}_{\mathrm{a}}\right)$ and the low velocity $\left(\mathrm{V}_{\mathrm{b}}\right)$ solution of a particular fragment emission. 
The advantages of using reverse kinematics are twofold. One the one hand, in normal kinematics it is very difficult to detect fragments having small velocities in the source frame. This problem is virtually eliminated in reverse kinematics, because the large velocity of the source dramatically increases the laboratory velocity of all fragments emitted in the center of mass. On the other hand, the large source velocity gives rise to a forward focusing of the reaction products, thus eliminating the need for a $4 \pi$ detection system. This effect also improves the efficiency of detecting coincidence fragments.

\subsection{Beams}

The $60 \mathrm{MeV} / \mathrm{A}{ }^{197} \mathrm{Au}$-ion beam was provided by the Lawrence Berkeley Laboratory BEVALAC Accelerator complex. The ${ }^{197} \mathrm{Au}$ ions were produced by the Abel injector at the SuperHILAC and accelerated to $1.2 \mathrm{MeV} / \mathrm{A}$. They were then stripped to a charge state of +61 and further accelerated to $8.5 \mathrm{MeV} / \mathrm{A}$ before being injected into the Bevatron. Here the ions reached their final energy of $60 \mathrm{MeV} / \mathrm{A}$.

The beam energy was determined from the radial position at extraction and from the synchrotron field, and is estimated to be known to $\pm 1 \%$. The beam spot was ellipsoidal with dimensions of about $1.8 \mathrm{~cm}$ in the vertical direction and $.9 \mathrm{~cm}$ in the horizontal direction. The extracted beam was delivered into the $60^{\prime \prime}$ diameter scattering chamber in 15 pulses or spills of particles per minute. The beam intensity on target varied between $1 \times 10^{6}$ and $3 \times 10^{7}$ particles/spill, with a typical spill length of 300 to 500 msec.

Besides the $\mathrm{Au}$ ion beam used for the data runs, ${ }^{14} \mathrm{~N},{ }^{28} \mathrm{Si},{ }^{56} \mathrm{Fe}$, and ${ }^{84} \mathrm{Kr}$ ion beams at $55 \mathrm{MeV} / \mathrm{A}$ were used for the calibration of the silicon detectors [Mc 86]. In order to minimize the time spent on calibrations the four ion beams were delivered at the same 
time by combining them into a single quadruplet beam. This was accomplished [Woz 93] by keeping the charge-to-mass ratio of all four ions the same at all stages of the acceleration from the ion source through the SuperHILAC to the Bevatron.

\subsection{Scattering chamber}

The nuclear reactions took place inside the 60 " diameter scattering chamber which was positioned at the end of the beamline. As is illustrated in figure 3.2, the chamber contained the Si array, the targets, and the scintillator paddle. To prevent interactions of the beam and reaction products with atmospheric gas atoms, a vacuum of $<10^{-5}$ torr was maintained inside the scattering chamber by means of a diffusion pump.

\subsection{Beam current monitor}

The beam current was monitored using a Faraday cup and a current integrator. The beam stop in the Faraday cup was operated at a potential of $+200 \mathrm{~V}$ to limit the scattering of electrons from the stop, which would give each beam particle a larger effective charge. The current integrator electronics were calibrated with a known current.

\subsection{Scintillator Paddle or Active Collimator}

A scintillator detector with dimensions $(20 \times 20) \mathrm{cm}^{2}$ was placed perpendicular to the beam direction. It was positioned $10 \mathrm{~cm}$ in front of the target ladder and a small 
aperture with dimensions $(1 \times 2) \mathrm{cm}^{2}$ in the middle of the paddle allowed the beam to pass through. The purpose of the paddle was twofold. First, it acted as a local collimator whose thickness $(.75 \mathrm{~cm})$ was sufficient to stop ${ }^{197} \mathrm{Au}$ beam particles along with any other high $\mathrm{Z}$ particles created upstream in the beam transport system. Second, it served as a veto counter allowing us to reject scattered beam particles outside the central beam spot.

\subsection{Si Array}

The apparatus used to detect the complex fragments consisted of 20 positionsensitive $\Delta E-E$ telescopes[Ke 92, Wa 90, Wa 87, Wa 78] that were arranged as shown in Figure 3.3. Each telescope contained a $300 \mu \mathrm{m}$ thick $\mathrm{Si} \Delta \mathrm{E}$ detector, followed by a 5 $\mathrm{mm} \mathrm{Si}(\mathrm{Li}) \mathrm{E}$ detector. The detector telescopes had dimensions $5.5 \mathrm{~cm} \times 5.7 \mathrm{~cm}$ and each telescope had an active area of $4.5 \mathrm{~cm} \times 4.5 \mathrm{~cm}$.

The telescopes were mounted on five $.4 \mathrm{~cm} \times 5.8 \mathrm{~cm} \times 38 \mathrm{~cm}$ strips made out of Lexan. They were positioned alternatively on the front and back sides of the strips in such a manner that the frames (dead areas) of the front detectors completely overlapped the frames (dead areas) of the detectors in the back as shown in Figure 3.4. In this way the dead areas along the vertical direction were reduced by almost $50 \%$. The dead areas of the detectors were further reduced by overlapping the Lexan strips along the horizontal direction as shown in Figure 3.3. The top, middle and bottom strips were positioned at $46 \mathrm{~cm}$ from the target, while the ones in between (second from bottom and top) were at $50 \mathrm{~cm}$. The maximum angular coverage in the lab subtended by the array was $\pm 16^{0}$. 
Each of the $300 \mu \mathrm{m}$ Si devices was an oxide passivated diffused-junction $n^{+} p$ fabricated from $5000 \Omega \mathrm{cm}$ p-type silicon. The devices were made at the Silicon Laboratory at LBL from 3-inch-diameter wafers with a thickness of $381 \pm 10 \mu \mathrm{m}$ and etched in stages to an average thickness of about $290 \mu \mathrm{m}$. Most of the detectors had a reasonably uniform thickness across their faces but some were found to have thickness nonuniformities of up to $10 \%$. In such cases an energy calibration beam was used to sweep across the face of the device in order to determine the nonuniformities, and thus apply the appropriate corrections. A reverse bias voltage of +50 to $+150 \mathrm{~V}$ was applied to each detector.

The $5000 \mu \mathrm{m}$ thick $\mathrm{Si}(\mathrm{Li})$ devices were lithium-drifted silicon diodes fabricated from $1000 \Omega \mathrm{cm}$ p-type silicon. They were produced by diffusing lithium onto the surface of a p-type silicon crystal and drifting it through the device to form a compensated region. These devices were also made at the Silicon Laboratory at LBL from 3 inch diameter wafers with a thickness of $4880 \pm 70 \mu \mathrm{m}$. The thickness nonuniformity of these devices was about $\pm 50 \mu \mathrm{m}(2 \%)$. In order to fully deplete these devices a reverse bias potential of +600 to $+700 \mathrm{~V}$ was applied to each detector.

Both types of silicon detectors $\mathrm{Si}$ and $\mathrm{Si}(\mathrm{Li})$ were position sensitive in one dimension. The front side of the devices $(300 \mu \mathrm{m} \mathrm{Si}$ and $5000 \mu \mathrm{m} \mathrm{Si}(\mathrm{Li}))$ was divided into $15 \mathrm{Au}$ (high conductivity) strips of $2.42 \mathrm{~mm}$ and 14 high resistivity gaps of .607 $\mathrm{mm}$ as shown in Figure 3.4 [Wa 90]. The conductive strips and the resistive gaps across the face of the detector provided us with the ability to determine the position of a particle which struck the detector by using the method of resistive charge division. Electrons created by the passage of a charged particle through the detector were collected at a Au ohmic contact ( $\mathrm{n}^{+}$contact) on the back face of the device while the holes were collected on the front ( $p$ contact). The total negative charge collected through the $n$ contact gave a measure of the total energy $E$ of the particle transversing or 
stopping in the detector, while the amount of positive charge collected through the resistor-divider $\mathrm{p}$ contact gave a signal $\mathrm{X}_{\mathrm{E}}$ proportional to the position. One of the two sides of the front face of the detector (the one always closest to the beam) was terminated to ground through $50 \Omega$ resistor so that the position $\mathrm{X}$ of the fragments was proportional to the hole signal divided by the electron signal $\left(X=X_{E} / E\right)$. Because all of the resistance appeared between the high conductivity strips, the position signals were discrete. Although the discrete nature of the devices made them self-calibrating, it also limited the position resolution to the width of a strip plus a gap (about $3 \mathrm{~mm}$ ). The strips of the $300 \mathrm{Si}$ were arranged orthogonally to the strips of the $5000 \mathrm{Si}(\mathrm{Li})$ within each telescope; this way both $\mathrm{X}$ and $\mathrm{Y}$ position signals were obtained for each charged particle that hit a telescope.

\subsection{Neutron Calorimeter}

So far, the main technique developed for neutron multiplicity measurements has been based [Pi 91, Cha 91, Jac 91, Que 91, Sch 91] on thermalizing the emitted neutrons in a large tank filled with a liquid scintillator doped with gadolinium and measuring the light flashes associated with the neutrons striking the detector. These tanks cover $4 \pi$ and achieve a very good efficiency for low-energy neutrons. However, they are inappropriate for reverse kinematic reactions, at intermediate and high energies (they suffer from the difficulty of thermalizing high energy-neutrons in a reasonable volume of scintillating liquid), where the bulk of the neutrons is emitted with approximately the beam velocity in a cone centered around the beam direction.

In this work we have utilized a novel neutron calorimetric approach [ $\mathrm{Pa} 88, \mathrm{~Pa} 90$ ] which is particularly suitable for the measurement of high multiplicities of neutrons of approximately the same energy. According to this approach, the integral response of a 
plastic scintillator to a number $\mathrm{N}$ of monoenergetic neutrons detected at the same time has a mean value equal to $\mathrm{N}$ times the mean response of a single neutron, and a dispersion approximately $\sqrt{\mathrm{N}}$ times the dispersion for a single neutron. If the neutron multiplicity $\mathrm{N}$ is sufficiently high, a reasonably low relative dispersion can be obtained, with a mean response proportional to the multiplicity N. Therefore high multiplicities of neutrons of approximately the same energy can be measured through the total light output response of a plastic-scintillator calorimeter. The condition of a low neutron energy spread was fulfilled in the present experiment by using reverse kinematics where the source velocities can be made much greater than the mean neutron velocity in the source frame.

The neutron detector (or neutron calorimeter) $[\mathrm{Pa} 88, \mathrm{~Pa} 90]$ consisted of $16 \mathrm{NE} 110$ plastic scintillator blocks and was installed behind the 60 -inch scattering chamber that contained the silicon array as is illustrated in Figure 3.1. Each scintillator block was $50 \mathrm{~cm} \times 25 \mathrm{~cm} \times 60 \mathrm{~cm}$ and was optically connected through the $50 \times 25 \mathrm{~cm}^{2}$ face to a EMI 9823B photomultiplier by means of a lucite guide. Total internal reflection of the scintillation light was obtained by preserving a plastic-air separation surface optically isolated from the environment. The modules were mounted as shown in figure 3.5 with a total depth of $50 \mathrm{~cm}$ and a distance from the target of $200 \mathrm{~cm}$ thus covering an angular range in the lab of $\pm 20^{\circ}$. The hole in the center of the neutron calorimeter through which the beam line passed was about $6 \%$ of the total area of the device. A $2 \mathrm{~mm}$ lead shield was placed in front of the calorimeter to reduce the number low-energy $\boldsymbol{\gamma}$-rays entering the detector; these $\gamma$-rays are characterized by energies of up to hundreds of $\mathrm{keV}$ and constitute the bulk of prompt emission. The lead shielding also helped to stop any fast light charged particles that penetrated the reaction chamber walls.

The neutron multiplicity was obtained from the light output response of the scintillators. The light output of a scintillator depends on the efficiency of the 
scintillator for converting ionization energy to photons. As radiation (neutrons and -rays) passes through the calorimeter, it excites the atoms and molecules of the scintillator material which in turn deexcite by emitting light. For high-energy neutrons, the atoms and molecules are excited (absorb energy) by the recoil proton in (n,p) scattering interactions. The reemission of energy in the form of visible light usually occurs immediately (within $10^{-8}$ seconds) after absorption; this process is known as fluorescence. Although other proccess, such as phosphorence can occur, in which the delay time between absorption and reemission may last anywhere from a few microseconds to hours depending on the material, prompt fluorescence represents most of the observed scintillation light .

The light is transmitted to the photomultiplier where it is converted into a weak current of photoelectrons which is further amplified by an electron-multiplier system. To separate the light output generated by the neutrons from the prompt $\gamma$-ray flash, we took advantage of their different light pulse shapes (which depend directly on their decay times). Since the neutrons are slower, the prompt $\gamma$-ray flash (fast signal) has decayed enough that it does not contaminate the neutron signal (slow signal) and thus by subtracting the fast from the slow signal one can obtain the light output response of the neutrons.

\subsection{Targets}

The targets chosen in this study are shown in table 3.1 along with their atomic number and thickness. They covered a wide range in $\mathrm{Z}$ from ${ }^{12} \mathrm{C}(\mathrm{Z}=6)$ all the way to ${ }^{197} \mathrm{Au}(\mathrm{Z}=79)$. The target thicknesses were chosen such that no more than $1 \%$ of the beam energy was lost in the target. The five targets were mounted simultaneously on a 
target ladder. The vertical position of the ladder was adjustable through a small d.c. motor controlled from the counting room, allowing the targets to be changed quickly without opening the scattering chamber.

\begin{tabular}{ccc} 
target & $\mathrm{Z}$ & $\mathrm{mg} / \mathrm{cm}^{2}$ \\
\hline $\mathrm{C}$ & 6 & 2.13 \\
$\mathrm{Al}$ & 13 & 1.79 \\
$\mathrm{~V}$ & 23 & 2.05 \\
$\mathrm{Cu}$ & 29 & 1.85 \\
$\mathrm{Au}$ & 79 & 2.01 \\
\hline
\end{tabular}

Table 3.1. Targets

\subsection{Electronics and Data Acquisition}

An event was characterized as valid and accepted for data processing only when it produced a "master gate". A "master gate" was a logic output signal that was generated when a coincidence signal was produced in at least one of the 20 telescopes of the Si-array. Each coincidence signal was the product of a logic AND between the $\Delta \mathrm{E}(300 \mu \mathrm{m} \mathrm{Si})-$ and the $\mathrm{E}(5000 \mu \mathrm{m} \mathrm{Si})$-electron signals (of a single telescope) with the additional time constraint that the E-signal had to arrive within $100 \mathrm{~ns}$ of the $\Delta \mathrm{E}$-signal. The signals from the neutron calorimeter were not included in generating the master gate but instead were passively recorded along with each valid event generated in the Si- array. Figure 3.6 is an electronics diagram illustrating the logic used to select the desired information from the signals produced by the $\mathrm{Si}$-array and neutron calorimeter. 
When a master gate was generated, the analog information stored in the ADCs and TDCs were digitized and read by a Starburst processor and transferred over a local ethernet to a VAX-3300, where it was written on tape. The constant fraction discriminator thresholds were set to exclude protons and alpha particles from triggering the acquisition system. In order to compress the data written on tape, a bit register was used to identify those telescopes that were actually hit by a fragment in each event.

For each telescope five signals were recorded on tape. These were position and energy signals from each of the $\Delta E$ and $E$ detectors along with a timing signal that was useful in identifying the fragments that originated from the same event.

For each of the sixteen modules of the neutron detector a fast and a slow signal was written on tape [for a total of ( 2 signals per module $) \times(16$ modules $)=32$ signals]. The fast signal was integrated over a period of $5 \mathrm{~ns}$ while the slow over $250 \mathrm{~ns}$. The two signals were used to discriminate between $\gamma$-rays and neutron emission. 


\section{Chapter 4}

\section{Calibrations}

\subsection{Position Calibrations}

As described in Chapter 3, the front face of each of the 300 and $5000 \mu \mathrm{m}$ Si-devices is divided into 15 strips and 14 gaps. A resistor chain across the front face of the devices is created by using strips of high conductivity material to separate gaps of high resistivity material. In this way particles incident on a single strip are characterized by the same raw position signal. Figure 4.1 shows density plots of the raw position $X_{D E}$ (and $Y_{E}$ ) signal plotted as a function of the raw energy DE (and $E$ ) signal for a 300(top) (and 5000(bottom)) $\mu \mathrm{m}$ Si device. These plots illustrate how the data looked on-line during the experiment. One clearly notices 15 diagonal lines which correspond to the 15 strips of the detector. The line with the lowest position signal value corresponds to the strip farthest away from the signal contact.

Within a telescope, the strips of the 300 and $5000 \mu \mathrm{m}$ Si-devices were oriented $90^{\circ}$ relative to each other in order to determine the $\mathrm{X}$ - and $\mathrm{Y}$ - position of the charged particles traversing the telescope. The position was determined by using the method 
described in Kaufman et al. [ Kau 70]. The position $X$ of a particle that hit a $\Delta E-S i$ detector was computed from the ratio:

$$
X=\frac{\left(X_{D E}-P_{0}\right)}{\left(E-E_{0}\right)}
$$

where

$\mathrm{X}$ is the distance of the particle from the grounded end of the telescope

$\mathrm{X}_{\mathrm{DE}}$ is the raw position signal,

$E$ is the raw energy signal,

$E_{o}$ and $P_{o}$ are the electronic base-line offsets (pedestals).

Similarly, for the E-Si detector, the position $\mathrm{Y}$ of a particle that hit an E-Si detector was given by the ratio:

$$
\mathrm{Y}=\frac{\left(\mathrm{Y}_{E}-\mathrm{P}_{\mathrm{O}}\right)}{\left(\mathrm{E}-\mathrm{E}_{\mathrm{O}}\right)}
$$

Typical spectra of $X$ and $Y$ for singles events are shown in Figures 4.2a and 4.2b respectively. The fifteen peaks correspond to the fifteen strips of the detector. From these plots the position $\mathrm{X}_{\text {channel }}$ (or $\mathrm{Y}_{\text {channel }}$ ) of the midpoint of each strip (in channel number) was determined from the average positions of the peaks. The position of the midpoint of each strip in millimeters $X_{\mathrm{mm}}$ (or $Y_{\mathrm{mm}}$ ) is a well-known value determined from the dimensions of the detectors. The $\mathrm{X}_{\mathrm{mm}}$ (or $\mathrm{Y}_{\mathrm{mm}}$ ) values were then plotted as a function of the average value $X_{\text {channel (or }} Y_{\text {channel }}$ ) and a best fit through the 15 points gave the desired relation : $X_{m m}=f\left(X_{\text {channel }}\right)$ or $Y_{m m}=f\left(Y_{\text {channel }}\right)$.

Since the width of a strip is $2.42 \mathrm{~mm}$ and that of a gap(between two strips) .607 $\mathrm{mm}$, the theoretical position resolution of the silicon detectors was calculated to be \pm $1.5 \mathrm{~mm}$. 


\subsection{Energy Calibrations}

The energy calibrations were done by exposing directly the detectors to low intensity beams ( $<100$ particles/s) of known energy. An extensive energy calibration of the silicon detectors was completed in a relatively short time, by using a quadruplet beam consisting of ${ }^{14} \mathrm{~N},{ }^{28} \mathrm{Si},{ }^{56} \mathrm{Fe},{ }^{84} \mathrm{Kr}$. In order to expose all of the detectors to the calibration beams, the in-plane telescopes were swept across the beam, then the array was raised or lowered remotely and the out-of-plane telescopes were swept through the beams.

The $5 \mathrm{~mm} \mathrm{Si}(\mathrm{Li})$ detectors were calibrated by having the quadruplet or cocktail beam run directly in the detector without the $300 \mu \mathrm{m} \mathrm{Si}$ in front. These detectors had sufficient depth to stop each of the $55 \mathrm{MeV} / \mathrm{A}$ ions of the cocktail beam. A typical raw ADC energy spectrum of the $55 \mathrm{MeV} / \mathrm{A}$ cocktail beam in a $5 \mathrm{~mm} \mathrm{Si}(\mathrm{Li})$ detector is shown in Figure 4.3a. The four peaks correspond to the measured energy of the ${ }^{14} \mathrm{~N}$, ${ }^{28} \mathrm{Si},{ }^{56} \mathrm{Fe},{ }^{84} \mathrm{Kr}$ ions; the energy is expressed in channel numbers. The energy $\mathrm{E}_{\mathrm{MeV}}$, in $\mathrm{MeV}$, associated with these peaks was obtained from $\mathrm{E}_{\mathrm{MeV}}=\mathrm{E}_{\text {beam }} * \mathrm{~N}-\mathrm{E}_{\text {phd }}$ where $E_{\text {beam }}$ is the beam energy per nucleon, $\mathrm{N}$ is the number of nucleons in each ion, and $E_{p h d}$ is a correction due to the pulse height defect. This last correction was less than $1 \%$ and will be discussed later in this section. The $\mathrm{E}_{\mathrm{MeV}}$ values were then plotted as a function of the mean value $E_{\text {channel }}$ of each peak and a best linear fit through the (four) points gave the desired relation:

$$
E_{\mathrm{MeV}}=\mathrm{a}+\mathrm{b} \mathrm{E}_{\text {channel }}
$$

between the deposited energy in $\mathrm{MeV}$ and the $\mathrm{ADC}$ channel number. 
The measured energy in the $5 \mathrm{~mm} \mathrm{Si}(\mathrm{Li})$ detectors, with and without the $300 \mu \mathrm{m}$ detectors in front, was then used to determine the energy $\Delta \mathrm{E}_{\mathrm{MeV}}$ (in $\mathrm{MeV}$ ) deposited in the $300 \mu \mathrm{m}$ Si detectors. Figure $4.3 \mathrm{~b}$ is a typical raw ADC spectrum of the cocktail beam in a $300 \mu \mathrm{m}$ Si detector. Again the energy $\Delta \mathrm{E}_{\mathrm{MeV}}$ for each ion was plotted as a function of the mean value $\Delta E_{\text {channel }}$ of each peak and a best linear fit through the points gave the desired relation.

There were a couple of $300 \mu \mathrm{m}$ detectors for which the ADC spectra were not characterized by sharp peaks like those of Figure $4.3 \mathrm{~b}$. The energy spectrum of the worst $300 \mu \mathrm{m} \mathrm{Si} \mathrm{detector} \mathrm{is} \mathrm{shown} \mathrm{in} \mathrm{Figure} \mathrm{4.4a.} \mathrm{The} \mathrm{broad} \mathrm{widths} \mathrm{and} \mathrm{ugly} \mathrm{shapes} \mathrm{of}$ the peaks are due to variations in thickness from one region of the detector to another. After correcting for the nonuniformities in thickness the shapes of the peaks improved considerably, as shown in Figure 4.4b. Correcting for the nonuniformities of the devices was extremely important, since our atomic number resolution (which is discussed in section 4.3) depends on the $300 \mu \mathrm{m}$ Si energy resolution.

Corrections for the energy losses in the $1.5 \mathrm{mg} / \mathrm{cm} \mathrm{Au}$ foils that were used for electron suppression as well as for losses in the target, were applied for each fragment, using range energy tables [Hub 80]. Corrections for the pulse-height defect in the $5 \mathrm{~mm}$ $\mathrm{Si}(\mathrm{Li})$ were performed for the calibration beam and for each detected fragment using the systematics of Moulton et al. [Mou 78]. The pulse-height defect (PHD) was calculated as a function of the measured energy of the ion deposited in the $5 \mathrm{~mm} \mathrm{Si}(\mathrm{Li})$ detectors and the charge of the ion. A detailed description of the simple power-law formula used is given in reference [Mou 78]. Since the PHD is thought of to be primarily an end-ofrange effect, the above correction was not applied to the $300 \mu \mathrm{m} \mathrm{Si}(\mathrm{Li})$ detectors since none of the identified particles stopped in these detectors. The energy calibration was estimated to be accurate to approximately $1.5 \%$. 


\subsection{Atomic Number Calibrations and Mass Parameterization}

\section{Atomic Number Calibrations}

The atomic number of the detected particles was determined from the measured $\Delta \mathrm{E}$ and $E$ values. Figure 4.5 is a plot of the energy lost $\Delta E$ in the $300 \mu \mathrm{m} \mathrm{Si}(\mathrm{Li})$ versus the energy deposited $\mathrm{E}$ in the $5 \mathrm{~mm} \mathrm{Si}(\mathrm{Li})$ for a single telescope. Here one clearly can identify an intensity pattern of alternating valleys and ridges, where each ridge corresponds to fragments with a different atomic number $Z$. The reason for this behavior can be traced to Bohr's classical formula ${ }^{2}$ which gives the energy that a particle loses when it travels a distance $\mathrm{dx}$ in some material medium. The formula is given by :

$$
-\frac{d E}{d x}=\frac{4 \pi z^{2} e^{4}}{m_{e} v^{2}} N_{e} \ln \frac{\gamma^{2} m_{e} v^{3}}{z e^{2} \omega}
$$

with

$\mathrm{N}_{\mathrm{c}}$ : density of electrons in material medium $\mathrm{Z}$ : atomic number of particle

e : elementary charge $\mathrm{m}_{\mathrm{e}}:$ electron mass

$\mathrm{v}$ : velocity of particle $\gamma: 1 / \sqrt{1-\beta^{2}}$

$\omega:$ mean orbital frequency of electrons $\beta: v / c$ of incident particle

By neglecting the slowly varying logarithm term in equation 4.4 we get:

$$
-\frac{d E}{d x} \propto \frac{Z^{2}}{v^{2}} \propto \frac{M Z^{2}}{E} \propto \frac{Z^{3}}{E}
$$

where the additional assumption that $\mathrm{Z} \propto \mathrm{M}$ has been made and $\mathrm{M}$ is the mass of the particle. By equating $d E / d x$ with $\Delta E$, the above equation (4.5) can be reduced to $\Delta E * E$ 
$\propto \mathrm{Z}^{3}$ which explains the hyperbola for each separate $\mathrm{Z}$-value in the $\Delta \mathrm{E}-\mathrm{E}$ plane (see Figure 4.5).

In the data analysis the 2-dimensional $\triangle E-E$ plots were transformed to 1 dimensional plots of $Z$ by means of the equation $Z=q((\Delta E+\alpha) E\}^{1 / 3}$ where $\alpha$ was a constant chosen by trial and error so as to straighten the curved Z-lines and $q$ was a scaling factor. Both of these constants $\alpha$ and $q$ have no physical significance. Figure $4.6 \mathrm{a}$ and $4.6 \mathrm{~b}$ are representative examples of charge spectra resulting from this procedure for two detectors, one at very small angles and the other at large angles. Each peak corresponds to a different element and in the case of the detector near the beam, peaks corresponding to atomic numbers up to $\mathrm{Z}=52$ are clearly visible (see Figure 4.6a.).

The accuracy of the Z-calibrations was determined by utilizing the quadruplet $(\mathrm{N}, \mathrm{Si}$, $\mathrm{Fe}, \mathrm{Kr}$ ) beam runs. In Figure 4.7 the peaks represent the $\mathrm{Z}$ values for $\mathrm{N}, \mathrm{Si}, \mathrm{Fe}$, and $\mathrm{Kr}$ as determined by the $\mathrm{Z}$-calibrations. The peaks reproduce the atomic number values of the $\mathrm{N}, \mathrm{Si}, \mathrm{Fe}$, and $\mathrm{Kr}$ within $0.1-0.2 \mathrm{Z}$ units.

\section{Mass Parameterization}

Estimating the average mass of the detected fragments is not a straightforward task since the primary fragments can have large excitation energies and thus evaporate a substantial number of light particles. In this work the mass $\mathrm{M}$ associated with each Zvalue was determined from the mass parameterization proposed in Reference [Cha 88]. Charity et al. [Cha 88] have utilized the statistical code PACE [Gav 80] to simulate this evaporation process for primary fragments over a large range of $Z, M$ and excitation energy. The average mass $M$ for each $Z$-value determined from the PACE simulations is given approximately by the relation $\mathrm{M}=2.08 \mathrm{Z}+0.0029 \mathrm{Z}^{2}$ which is in agreement 
with experimentally determined average masses [Aug 87]. Over the range of fragments $5<\mathrm{Z}<40$, it is estimated to be accurate to within $\pm 0.5 \mathrm{amu}$.

\subsubsection{Neutron Calorimeter Calibrations.}

\section{Channel number to MeVee transformations}

The quantity of light produced in an organic scintillator is generally expressed in MeVee, i.e. the electron energy $L_{k}(E)$ which would produce the same amount of light as the particle $\mathrm{K}$ at energy $\mathrm{E}$. The light output signals of each module were transformed from channel number to $\mathrm{MeVee}(\mathrm{MeV}$ electron equivalent) by utilizing three calibration points. A $1 \mathrm{MeVee}$ calibration point was obtained by using the radioactive source ${ }^{60} \mathrm{Co}$. Cobalt -60 emits two gamma rays of $1.17 \mathrm{MeV}$ and $1.33 \mathrm{MeV}$ that produce a Compton edge at an energy of approximately $1 \mathrm{MeV}$. An $8 \mathrm{MeVee}$ calibrating point was obtained by using $14 \mathrm{MeV}$ neutrons that were produced in the reaction ${ }^{3} \mathrm{H}\left({ }^{2} \mathrm{H}\right.$, $\left.{ }^{2} \mathrm{He}\right) \mathrm{n}$ induced by $200 \mathrm{keV}$ deuterons[ $\left.\mathrm{Pa} 90\right]$. Finally, a high-amplitude calibration point of about $40.7 \mathrm{MeVee}$, easily obtainable in all labs, was provided by the cosmic rays[Pa 90]. A linear transformation between channels and MeVee was obtained for all (16) modules. The neutron calorimeter was calibrated off-line before the acquisition of any data.

As was discussed in section 3.9 for each of the sixteen modules a fast and a slow signal was recorded; the fast signal was integrated over a period of $5 \mathrm{~ns}$ while the slow signal over $250 \mathrm{~ns}$. The two signals were used to discriminate between $\gamma$-rays and neutrons. Since the neutrons are slower, the prompt $\gamma$-ray flash (fast signal) should decay substantially that it does not contaminate the neutron signal (slow signal). Thus by subtracting the fast from the slow signal, one can obtain the light output response of 
the neutrons. The neutron light output for each module was determined by subtracting the fast signal from the slow signal. The total neutron light output was then obtained by summing the neutron light output of all modules. Figure 4.8 is a representative spectrum of the total neutron light output, in MeVee, obtained from the $60 \mathrm{MeV} / \mathrm{A} \mathrm{Au}$ $+\mathrm{Cu}$ reactions.

\section{MeVee to neutron multiplicity transformations}

The MeVee scale was converted into neutron multiplicity by utilizing the Monte Carlo code of Ref. [Ang 79] that simulates the light output response produced by neutrons on their way through an organic scintillator. In this code, which for convenience we will refer to as the NC (Neutron Calorimeter) code, the total neutron interaction processes considered are the elastic scattering of neutrons by ${ }^{12} \mathrm{C}$ and by protons along with the following inelastic scattering processes: ${ }^{12} \mathrm{C}(\mathrm{n}, \mathrm{n} \gamma){ }^{12} \mathrm{C}^{*}$, $\left.{ }^{12} \mathrm{C}(\mathrm{n}, \alpha){ }^{9} \mathrm{Be},{ }^{12} \mathrm{C}\left(\mathrm{n}, \mathrm{n}^{\prime}\right) 3 \alpha,{ }^{12} \mathrm{C}(\mathrm{n}, \mathrm{p})\right)^{12} \mathrm{~B},{ }^{12} \mathrm{C}(\mathrm{n}, \mathrm{d})^{11} \mathrm{~B}$. The $\mathrm{NC}$ code has been tested $[\mathrm{Pa}$ 90] by reproducing the light output response of a plastic module identical to the ones used in this experiment. Figure 4.9, taken from reference [ $\mathrm{Pa} 90]$, shows the light yield distributions from a NE110 plastic scintillator block for both simulated and experimental data. The light yield distributions were obtained with mono-energetic neutron $(14 \mathrm{MeV})$ multiplicities of 10,20 , and 30 and were constructed by summing the light yields for the same number of simulated and experimental events. Good agreement is obtained between the experimental and calculated distributions.

The NC code was modified to include the geometry of the neutron calorimeter detector and simulations were performed for each of the Au - induced reactions at different impact parameters. To illustrate our approach, we consider here as a representative example the calculations that were done for the ${ }^{197} \mathrm{Au}+{ }^{63} \mathrm{Cu}$ system. The energy and angular distributions of the neutrons emitted at different impact 
parameters $(b=0,3,6,9)$ for the $\mathrm{Au}+\mathrm{Cu}$ reaction were calculated with the Monte Carlo code (BNV + GEMINI) which is discussed in chapter 6 . These distributions were then used as input parameters in the NC code and the light output response of the neutron calorimeter was obtained at different impact parameters of the reaction. The number of neutrons emitted at each impact parameter was also obtained from the BNV+GEMINI calculations. The average total number of neutrons emitted at each impact parameter was plotted as a function of the average total light output response (see Figure 4.10) and the best fit through the points gave the desired relationship between neutron multiplicity and MeVee. However, as can be seen from Figure 4.10 the neutron light output response for central collisions (60 neutrons) is predicted to be as high as $300 \mathrm{MeVee}$ while in our experimental spectra the maximum light output extends out only to about 70 MeVee. Although much effort was spent in checking and rechecking the calibrations, the origin of this discrepancy is not fully clear. Some of the possible errors associated with this discrepancy will be discussed in the following section. In order for the simulations to reproduce the range of the experimental data a factor of $\sim 4.5$ was used to scale down the predicted light output. Such a procedure may not affect substantially our results (qualitatively or quantitatively), since the maximum and minimum number of neutronss are assigned to the upper and lower ends of the spectra.

\subsubsection{Possible errors}

Although there may be several possible sources of error that contribute to the observed discrepancy between the experimental data and the simulations, we will discuss here two that seem to be the most obvious. 
One source of error arises from the fact that the neutron calibrations are modeldependent. That is, the neutron distributions depend on the predicted angle and kinetic energy of the fragments from which the neutrons are emitted. A slight change (a few degrees in the lab) of the angle of the fragments (or fragment) along with, for example, a $15 \%$ change in their kinetic energy can result in a $20-25 \%$ variation in the simulated light output. In addition, the total number of neutrons, predicted by running the simulations several times at impact parameter $b=0$, was found to vary by as much as $7 \%$. However, these errors are small and cannot account for the factor of 4.5 that was utilized to scale down the predicted light output

On the other hand, a significant source of error could arise from the subtraction method we used to determine the experimental neutron light output. As discussed in the previous chapter, the neutron light output was obtained by subtracting the fast signal that was integrated over $5 \mathrm{~ns}$ from the slow signal that was integrated over $250 \mathrm{~ns}$. Estimating the uncertainty in the subtraction process is difficult because one has to know the exact time relation between the integration gates and each individual signal. In this experiment all signals were adjusted in time within $1 \mathrm{~ns}$ from each other; this is the best one can do with delay lines. However, considering that the gamma flash from the plastic is of the order of a few nsec, a 1 nsec jitter could be a source of significant error. Although it is difficult to determine the percentage of the signal that can arise from a 1 ns jitter, a test to determine the sensitivity of the subtraction was performed by subtracting $85 \%$ of the experimentally determined fast signal from the slow signal \{slow\}-.85 fast (instead of $\{$ slow $\}-\{$ fast $\}$ ). This procedure resulied in values of the light output as large as $120 \mathrm{MeVee}$ that can account for a factor of 2 in our calibrations. 


\section{Chapter 5}

\section{Results}

In this chapter the experimental results from the $\mathrm{Au}+\mathrm{C}, \mathrm{Al}, \mathrm{V}, \mathrm{Cu}$, and $\mathrm{Au}$ reactions at $60 \mathrm{MeV} / \mathrm{A}$ are presented. Initially (section 5.1), velocity spectra, angular distributions and cross sections from the inclusive data are shown. Subsequently, in sections 5.2 and 5.3, the coincidence data from the Si-array and the neutron calorimeter are discussed.

\subsection{INCLUSIVE DATA}

\subsubsection{Invariant cross section plots $(V \perp-V I I$ diagrams).}

The emission of complex fragments in the $60 \mathrm{MeV} / \mathrm{A} \mathrm{Au}$-induced reactions can be studied by plotting, for a given fragment $\mathrm{Z}$, the Galilean invariant cross section in velocity space. The Galilean invariant cross-section is given by $\frac{\partial^{3} \sigma}{V_{\perp} \partial V_{\perp} \partial V_{\|} \partial \phi}$ and can be simplified to $\frac{\partial^{2} \sigma}{V_{\perp} \partial V_{\perp} \partial V_{\|}}$by integrating over the angle $\phi$; the $2 \pi$ factor that results 
fiom the integration is a constant and can be left out. Now, for fragments that arise in the statistical decay of a single source with high angular momentum, the differential cross sections are expected to be isotropic in the reaction plane $(\mathrm{d} \sigma / \mathrm{d} \Omega \propto 1 / \sin \theta \Rightarrow$ $d \sigma / d \theta=$ constant, where $\theta$ is the emission angle in the source frame $)^{3}$. In order to examine the emission pattern of the complex fragments in the reaction plane we have multiplied $\frac{\partial^{2} \sigma}{V_{\perp} \partial V_{\perp} \partial V_{\|}}$by $V_{\perp}=V \sin \theta$, where $\theta$ and $V$ are the emission angle and velocity in the source frame, respectively.

In this analysis, $\frac{\partial^{2} \sigma}{\partial V_{\perp} \partial V_{\|}}$cross-sections in the $V_{\perp}-V_{\|}$plane were constructed for each detected fragment from the $60 \mathrm{MeV} / \mathrm{A} \mathrm{Au}+\mathrm{C}, \mathrm{Al}, \mathrm{V}, \mathrm{Cu}$, and $\mathrm{Au}$ reactions. It may be helpful before presenting the data, to gain an understanding of the $V_{\perp}-V_{\|}$ diagrams by discussing as an example, the invariant cross section $\partial^{2} \sigma / \partial V_{\perp} \partial V_{\|}$that results from a well known mechanism.

\section{$\mathrm{V} \perp$ - VII diagram for Compound Nucleus Decay}

Figure 5.1 is a schematic representation of the cross-section $\partial^{2} \sigma / \partial \mathrm{V}_{\perp} \partial \mathrm{V}_{\|}$for complex fragments emitted in a particular asymmetric binary decay from a compound nucleus. The two rings, represented by the shaded circular areas, correspond to the two fragments which are emitted with Coulomb- like velocities in the center-of-mass of the source; they are usually referred to as Coulomb circles because of their origin. The isotropic distributions along the Coulomb rings indicate that the fragments are emitted isotropically in the reaction plane from a single source with high angular momentum. The dashed lines correspond to the limits of the detector acceptance at $2.5^{\circ}$ and $16^{\circ}$ in the laboratory and the shaded area between the dashed lines is the cross section arising from the detected events. 
The center of these rings defines the laboratory velocity Vs of the source, and the radii $V_{1}$ and $V_{2}$ are the velocities with which the complex fragments are emitted in the source frame. The velocities $V_{1}$ and $V_{2}$ are not independent, but result from momentum conservation $\left(m_{1} V_{1}=m_{2} V_{2}\right.$, where $m_{1}$ and $m 2$ are the masses of fragments 1 and 2); momentum conservation requires that the fragments be emitted at $180^{\circ}$ with respect to each other, in general on different rings whose radii depend upon the Coulomb repulsion energy and the mass ratio of the fragments. In the special case of symmetric decay $V_{1}=V_{2}$, and the two rings overlap.

The width of the rings may arise from both fluctuations in the velocity of the primary fragments [Mor 75] and from the recoil effects associated with the sequential evaporation of light particles from these fragments. Furthermore, if $m_{1}<m_{2}$, the width of the ring associated with fragment 2 is expected to be narrower than its counterpart. This is because any fluctuation $d V_{1}$ in $V_{1}$ would result in a $d V_{2}=m_{1} / m_{2} d V_{1}$ change in the velocity $V_{2}$ of fragment 2 . In this relation $m_{1} / m_{2}<1$ and therefore $d V_{2}<d V_{1}$.

\section{Velocity Diagrams for the $60 \mathrm{MeV} / \mathrm{A} \mathrm{Au}$-induced reactions}

The experimental velocity of a complex fragment was determined from its measured kinetic energy using the mass parameterization discussed in section 4.3. From these velocities along with the measured scattering angles, contour plots of $\partial^{2} \sigma /$ $\partial V_{\perp} \partial V_{\|}$in the $V_{\perp}-V_{\|}$plane were constructed for each element. Representative examples of these contour plots for the $60 \mathrm{MeV} / \mathrm{A}{ }^{197} \mathrm{Au}+{ }^{12} \mathrm{C}$ reaction are shown in Figure 5.2a. A ring of high cross section (Coulomb ring) is visible in all plots. In contrast the central region is characterized by an absence of events. The relative sharpness of the rings indicates that the fragments may be emitted from the binary decay of a single equilibrated source. Although the complex fragments can be emittcd from a range of sources formed in incomplete fusion processes (incomplete fusion was 
discussed in Chapter 1), the small size of the carbon target limits the range of mass transfers that can occur from the target to the projectile. Therefore the sources that can be formed in the $\mathrm{Au}+\mathrm{C}$ reactions are very similar in size with each other and can be effectively associated with a "single" source as seen from Figure 5.2a. The distributions along the Coulomb rings appear isotropic and suggest that the fragments are emitted isotropically in the reaction plane from a "single" source with high angular momentum. Similar Coulomb rings have been observed at low bombarding energies [ $\mathrm{Ch} 88 \mathrm{a}, \mathrm{Ch}$ $88 \mathrm{~b}, \mathrm{Ch} 90 \mathrm{]}$ and have been attributed to the binary decay of a compound nucleus arising either from complete or incomplete fusion.

The center of each ring corresponds to the velocity of the source from which the fragments are emitted, and is indicated by the arrowhead in each subplot (See Fig. 5.2ae). Details on how the source velocity was determined will be given in section 5.1.2. The radii of the rings correspond to the emission velocities with which the fragments are emitted in the source frame. It can be observed that the radii decrease with increasing atomic number. This is due to linear momentum conservation in the centerof-mass which requires the lighter fragments to have larger velocities (see section 5.1.3). The width of the rings may arise from various processes and was discussed in the previous section.

Representative cross-sections in velocity space for the $60 \mathrm{MeV} / \mathrm{A} \mathrm{Au}+\mathrm{Al}, \mathrm{V}, \mathrm{Cu}$, and $\mathrm{Au}$ reactions are shown in Figures $5.2 \mathrm{~b}-\mathrm{e}$. In these plots the cross-sections do not have the well defined ring shaped distributions that were observed for the $\mathrm{Au}+\mathrm{C}$ reactions. Instead, they appear as filled-in oval distributions. A variety of factors could explain this filling-in. For instance the larger size of the targets provides a broader range of impact parameters and therefore a broader range of mass transfers. A large range of mass transfer produces a large range of source velocities, which can effectively smear out the ring along the $\mathrm{V}_{\|}$direction. In addition significant multifragment decay 
could lead to the filling-in of the rings from both sequential and simultaneous processes; in these processes the average emission velocities of each fragment are expected to decrease. Similar filled-in Coulomb circles have been also observed in ref. [Rou 93] and have been attributed to the large range of velocities and sizes of the sources produced in the incomplete fusion process [Bo 91, Co 89].

\subsubsection{Source Velocities}

The velocity of the source or sources from which the complex fragments are emitted can be obtained from the Coulomb rings by determining their centers. In references [Ch 88a, Ch 88b, De 91] the centers were extracted by fitting each of the Coulomb rings to a circle. In the present study however, the filling-in of the Coulomb rings, especially for the heavier targets, makes it difficult to extract the source velocities $V_{s}$ from the inclusive data with this technique. Instead, the source velocities (arrowheads in Fig. 5.2a-e) were determined from the 2 -fold events by applying the following expression:

$$
\mathrm{V}_{\mathrm{s}}=\frac{\sum \mathrm{m}_{\mathrm{i}} \mathrm{v}_{\mathrm{i}}}{\Sigma \mathrm{m}_{\mathrm{i}}}
$$

where $m_{i}$ and $v_{i}$ are respectively the mass and the velocity in the laboratory frame of the $\mathrm{i}$-th fragment, and the summation is performed over the two detected fragments. The source velocity Vs can be determined also by utilizing the higher $n$-fold events $(n=3,4$, and 5$)$. However since the binary events comprise $80 \%$ of all the coincidence data, it may be reasonable to associate the source velocity of the singles events with only the Vs of the 2-fold events. The coincidence data will be discussed in great detail in section 5.2. 
The average source velocities, determined by means of equation 5.1, for the 60 $\mathrm{MeV} / \mathrm{A} \mathrm{Au}+\mathrm{C}, \mathrm{Al}, \mathrm{V}, \mathrm{Cu}$ and $\mathrm{Au}$ reactions are shown in Figure 5.3. The statistical error in $V_{s}$ is smaller than the size of the symbols used in Figure 5.3. The large single error bar gives an estimate of the systematic error arising from the energy calibration of the detectors and the mass parameterization.

The distribution $\mathrm{V}_{\mathrm{s}}$ of the extracted source velocities is practically independent of the size of the target, with the exception of the distribution $V_{s}$ from the $A u+C$ reactions, which is slightly higher (about $2 \%$ ).

The extracted source velocities, for all targets, increase as a function of atomic number, peak around $\mathrm{Z}=40$ and then fall off. Such a dependence suggests that the fragments may be produced by a range of sources formed in incomplete fusion processes. For example the complex fragments with the highest source velocities are emitted from sources that are formed in peripheral collisions. In these reactions a small number of nucleons can be transferred from the target to the Au projectile without substantially reducing the velocity of the resulting source, but providing it with sufficient excitation energy to fission. Although other exit channels in the $60 \mathrm{MeV} / \mathrm{A}$ Au-induced reactions are possible, the emission of complex fragments around $\mathrm{Z} 40$ is favored with respect to the emission of other fragments between $4<\mathrm{Z}<75$. This is because complex fragments around $Z \sim 40$ are associated with the lowest conditional barriers in this fragment range $(4<\mathrm{Z}<75)$. The conditional barriers as predicted by the RFRM [Sie 86, Kra 79, Sie 85] for the binary decay of Au are shown in Figure 5.4; a minimum in the saddle point energy is observed around $Z \sim 40$. The process described above is similar to traditional fission, where a heavy nucleus ruptures into two fragments of approximately the same size after receiving a moderate amount of excitation energy. 
The Au-like sources formed in the peripheral collisions are characterized by large $V s$ values and favor the emission of complex fragments around $Z \sim 40$ with respect to the emission of other fragments in the range $4<Z<75$. Fragments associated with lower Vs values may arise from sources formed in more central collisions. In these reactions the projectile picks up more mass from the target and slows down to a greater degree.

\subsubsection{Emission Velocities}

The average emission velocity $V_{e}$ for each fragment was determined from $\left\langle V_{e}\right\rangle=$ $\left\langle\left|V_{\text {lab }}-\left\langle V_{\mathbf{s}}\right\rangle\right|>\right.$ where $V_{\text {lab }}$ is the velocity of the fragment in the laboratory frame and $\mathrm{V}_{\mathrm{s}}$ is the average source velocity as determined in section 5.1.2. In figure 5.5 we have plotted the average emission velocity as a function of the charge of the detected fragments. The emission velocity distributions of all targets are very similar to each other. Furthermore the average emission velocities display an almost linear decrease with increasing $Z$-value. Such a dependence is expected for Coulomb-like velocities ${ }^{4}$.

Additional evidence for the Coulomb-like nature of the emission velocities is provided by a simple calculation. The sharp Coulomb rings in the $\mathrm{Au}+\mathrm{C}$ reactions along with the small range of mass transfers that are possible from the target to the projectile suggest that the fragments in these reactions should arise from the binary decay of Au-like nuclei. The predicted emission velocities of fragments emitted in the binary decay of a Au nucleus are shown in Figure 5.5 by the solid lines. The predictions are based upon the Viola fission-fragment kinetic-energy systematics [Vi

85]. The Viola systematics gives the kinetic energy of the two fission fragments as a function of the $\mathrm{Z}$ and $\mathrm{A}$ of the fissioning system: 


$$
E_{\text {viola }}=0.1189 \mathrm{Z}^{2} / \mathrm{A}^{1 / 3}+7.3 \mathrm{MeV}
$$

Since the Viola systematics has been compiled for symmetric fission, it was generalized for asymmetric decay by solving for the radius parameter ro in

$$
E_{\text {Viola }}=1.44 \frac{Z_{1} * Z_{2}}{r_{0}\left(A^{1 / 3}+A^{1 / 3}\right)}
$$

with $Z_{1}=Z_{2}$ and $A_{1}=A_{2}$. The extracted $r_{0}$ was then used in equation 5.3 to calculate the kinetic energy $E_{v i o l a}$ released and the fragment velocities for asymmetric decays where $Z_{1} \neq Z_{2}$ and $A_{1} \neq A_{2}$. Excellent agreement is obtained between the experimentally determined and predicted emission velocities in the $\mathrm{Au}+\mathrm{C}$ reactions, for most of the detected fragments, confirming the Coulomb-like nature of the emission velocities.

The predicted emission velocities of fragments emitted in the binary decay of a $\mathrm{Au}$ nucleus were also compared with the experimental emission velocities determined for the heavier targets. The overall agreement (see Figure 5.5) indicates that the experimentally determined emission velocities are also Coulomb-like, and that the mass and charge of the decaying system do not depend strongly on the target. It seems that most of these fragments arise from fission of Au-like projectiles produced in peripheral collisions.

\subsubsection{Angular Distributions}

The experimental angular distributions $d \sigma / d \theta$ of complex fragments can be very useful for differentiating between equilibrium and non-equilibrium emission processes. For example, the flat angular distributions ( $\mathrm{d} \sigma / \mathrm{d} \theta=$ const.) observed at lower energies 
[Ch 88a-b], were shown to result from the binary decay of a compound nucleus (equilibrium process) formed in a complete or incomplete fusion reaction. In contrast, the experimental angular distributions $\mathrm{d} \sigma / \mathrm{d} \theta$ of projectile-like fragments and target-like fragments produced in non-equilibrium processes (deep inelastic/quasi elastic processes) showed a forward and backward peaking, respectively [Ch 88a-b]; as was discussed in Chapter 1 side peaking has also been observed.

Representative angular distributions of the fragments emitted in the $60 \mathrm{MeV} / \mathrm{A}$ ${ }^{197} \mathrm{Au}+{ }^{12} \mathrm{C},{ }^{27} \mathrm{Al},{ }^{51} \mathrm{~V},{ }^{63} \mathrm{Cu}$, and ${ }^{197} \mathrm{Au}$ reactions are shown in Figures 5.6a-e. The angular distributions were constructed in a frame moving with the average source velocity discussed in section 5.1.2. For a wide range of intermediate $\mathrm{Z}$ values $\mathrm{Z}=15$ 53) from the very asymmetric ${ }^{197} \mathrm{Au}+{ }^{12} \mathrm{C}$ system to the symmetric ${ }^{197} \mathrm{Au}+{ }^{197} \mathrm{Au}$ system, the angular distributions are approximately constant in $d \sigma / d \theta$. This suggests that the fragments may be emitted isotropically in the reaction plane from compound nucleus sources. The compound nucleus sources can be formed either in complete or incomplete fusion reactions.

However, for lighter $(Z<15)$ and heavier $(Z>53)$ fragments, the angular distributions show some backward- and forward-peaking component, respectively, which can be associated with the target- and projectile-like fragments produced in these reactions. These anisotropic components have already been observed previously, and have been interpreted as due to processes similar to the ones that occur in low-energy quasi-elastic and deep-inelastic reactions [ $\mathrm{Ch} 88 \mathrm{a}-\mathrm{b}, \mathrm{Ch} 90$ ]. The lines drawn in Figures 5.6a-e are the best fits through the points using second-order polynomials and were used to determine the cross section (see section 5.1.5). The statistical error associated with each point is smaller than the size of the symbol used. 


\subsubsection{Cross Sections}

The absolute cross section for each atomic number can be obtained by integrating the differential cross-sections $d \sigma / d \theta$ between $0^{0}$ and $180^{\circ}$. Since the experimental differential cross-sections $d \sigma / d \theta$ ranged from $25^{\circ}$ to $160^{\circ}$, the integrations were performed by fitting the angular distributions to second-order polynomials and then integrating the resulting fits over the full angular range. Over the measured angular range, the fitting polynomials were found to reproduce the measured distributions within 5\%. The angle-integrated cross-sections determined with the above procedure are plotted in Figure 5.7 as a function of fragment $\mathrm{Z}$-value, for all five targets. The statistical errors associated with these points are smaller then the size of the symbol used. The possible systematic error associated with the absolute cross section determination (target thickness, charge state of the beam particles, etc...) was estimated to be around $30 \%$. Such errors should not change the overall trends of the extracted charge distributions.

The absolute cross-sections as a function of atomic number $\mathbf{Z}$ are shown in Figure 5.7 for all reactions. In the case of the carbon target, the charge distribution peaks around $\mathrm{Z}=40$ and then drops off. This shape of the charge distribution is consistent with statistical emission from a compound system above the Businaro-Gallone transition point [ $\mathrm{Bu} 55]$. In particular the symmetric shape of the distribution (around $\mathrm{Z} 40$ ) suggests that the complex fragments are emitted from the compound binary decay of Au-like nuclei. Although the complex fragments may be emitted from a range of sources formed in incomplete fusion processes, the small size of the carbon target limits the range of mass transfers that can occur from the target to the projectile. Therefore the sources that can be formed in the $\mathrm{Au}+\mathrm{C}$ reactions will be very similar in size with the Au-like projectile. 
The conditional barriers for the binary decay of $\mathrm{Au}$, predicted by the Rotating Finite Range Model [Sie 86, Kra 79, Sie 85], were presented earlier in Figure 5.4. As can be seen, the cross section of the complex fragments from the $\mathrm{Au}+\mathrm{C}$ reaction is a reflection of the conditional saddle points. This result should be expected, since the relationship between the yield $Y$ and the conditional barrier $B(z)$ for a given complex fragment is given approximately by $Y \propto \exp \left(\left(-B(z) / T_{z}\right)\right)$ where $T_{z}$ is the saddle temperature.

For the heavier targets however, the shape of the charge distributions cannot be associated completely with statistical emission from systems either above or below the Businaro Gallone point. The distributions, for all heavy targets, decrease monotonically with increasing $Z$ to about $Z=20$, then gradually begin to increase, peak around $Z=40$ and then drop off again (see Figure 5.7). The peak around Z 40, present in the charge distributions of the $60 \mathrm{MeV} / \mathrm{A} \mathrm{Au}+\mathrm{Al}, \mathrm{V}, \mathrm{Cu}$ and $\mathrm{Au}$ reactions, is reminiscent of the fission peak observed in the $\mathrm{Au}+\mathrm{C}$ reactions. It suggests that a substantial portion of the fragments produced in these reactions may be attributed to the decay of Au-like sources produced in peripheral reactions.

However, a significant yield of lighter fragments $(Z<25)$ also present, indicates that other mechanisms may be responsible for the production of complex fragments. For instance, the yields of the lighter fragments may arise from multibody decay. The yield of lighter fragments is comparable in magnitude to the fission fragment yields ( $\mathrm{Z}$ $\sim 40$ ), and increases as a function of the mass of the target. This behavior suggests that multifragmentation may become increasingly important with increasing target size. This is because the larger range of mass transfers available from the heavier targets can be effectively associated with a larger range of excitation energies and multifragmentation is predicted to become increasingly important at higher energies (see Chapter 2). 


\subsection{COINCIDENCE DATA (FROM Si ARRAY)}

\subsubsection{Z-distributions selected by fragment size}

The coincidence data included events in which two or more fragments were detected simultaneously. The maximum number of fragments per event presented in this work is equal to 5 . Although six-fold events were also detected their substantially low number made it statistically impossible to obtain useful information.

Each of the fragments considered in the analysis had an atomic number $Z>5$. Fragments with atomic numbers less than/or equal to five were not included because of the detector threshold.

In Figures 5.8a-e we have plotted the charge distributions for each of the fragments comprising the $\mathrm{n}$-fold events in descending order according to their size. For example, the first frame of the top row includes the largest fragments emitted in binary decays and the second frame the smallest. In the second row, where 3-fold fragments are displayed, the first frame includes the largest fragment per event, the frame in the middle the second largest and the last frame the smallest, and so on. Each of the distributions was normalized to the same maximum number of counts for visual display purposes only. Therefore the areas under the distributions are not quantitatively correct.

In the case of the carbon target, most of the 2 -fold events arise from the fission of the Au-like projectiles, and both frames are dominated by a single sharp peak centered about $\mathrm{Z}=39$. For the 3 -body events we observe that the first two frames are occupied by sharp distributions centered about $\mathrm{Z}=38$, while in the last frame the distribution is 
narrower and centered at considerably lower values. This suggests that in these events two large fragments of approximately the same size are emitted along with a small one. For the 4-fold events we cannot discern a pattern of distribution for the fragments due to the extremely low number of events.

For the heavier targets and in particular for $\mathrm{Al}, \mathrm{V}, \mathrm{Cu}$, the distributions of the fragments, shown in Figure 5.8b-d, are nearly identical. The distributions associated with the 2 -fold events are characterized by peaks centered around $Z=39$ that arise from the fission of the Au-like projectiles produced in peripheral collisions and extend to lower $\mathrm{Z}$-values. For the 3 -fold events there is no preferential combination of emission, as was for the carbon target. The distributions shift to lower values and become narrower from the leftmost to the rightmost frame. The same pattern is observed for the 4-fold and 5-fold events. Finally, for the Au target (see Figure 5.8e) most of the fragments are located at low $\mathrm{Z}$-values, suggesting that these reactions lead predominantly to the production of small fragments.

\subsection{2 $V_{\text {source }}-Z_{\text {total }}$ plots}

\section{a) Experimental Data}

In the upper right quadrant of figures $5.9 \mathrm{a}-\mathrm{e} \mathrm{V}_{\text {source }}-\mathrm{Z}_{\text {total }}$ plots are shown for the $\mathrm{n}$-fold events (with $\mathrm{n}=2,3,4$, and 5) arising in the $60 \mathrm{MeV} / \mathrm{A} \mathrm{Au}+\mathrm{C}, \mathrm{Al}, \mathrm{V}, \mathrm{Cu}$ and $\mathrm{Au}$ reactions. The source velocity was reconstructed on an event-by-event basis from the velocities and the masses of the detected fragments, by means of eq. 5.1.

The data shown in these figures, especially for the heavier targets, can be understood by examining first the simpler pattern presented by the $\mathrm{Au}+\mathrm{C}$ system. For this system, the contour lines (shown in Fig. 5.9a II) are sharply localized in the $\mathrm{V}_{\text {source }}$ 
vs. $Z_{\text {total }}$ plane, and the values around which they are centered can be determined by projecting the data along the $\mathrm{Z}_{\text {tot }}$ (Fig. 5.9a) and $\mathrm{V}_{\text {source }}$ axes. The source velocity $\mathrm{V}_{\text {source }}$ peak is centered around $10.6 \mathrm{~cm} / \mathrm{ns}$, which is close to the beam velocity $(10.7$ $\mathrm{cm} / \mathrm{ns}$ ). The top arrow shown in figure $5.9 \mathrm{a}$ indicates the value of the beam velocity, while the bottom arrow corresponds to the source velocity value arising from complete fusicn. The narrow width of the $\mathrm{V}_{\text {source }}$ distribution is suggestive of the small range of impact parameters associated with the size of the carbon target; the smaller the target, the smaller the range of incomplete fusion products and therefore the smaller the range of source velocities. The total detected charge $Z_{\text {total }}$ distribution peaks around $Z=79$, which is the atomic number of the Au projectile. The narrow width of the peak along with the high cross section for $\mathrm{Z}=38$ \& 39 fragments (see section 5.1 .5 ) and the predominant binary nature of the coincidence events indicates that most of the events arise from the fission of the Au-like projectile.

In the upper right quadrant of figures $5.9 \mathrm{~b}-\mathrm{e}$ the cross section in the $\mathrm{V}_{\text {source }} \mathrm{vs}$. $\mathrm{Z}_{\text {total }}$ plane is shown for coincidence events arising from the reactions of the Au projectile with the heavier targets. The events in these reactions, unlike those in the $\mathrm{Au}+\mathrm{C}$ reactions, are not sharply localized around a point, but are dispersed over a large range along both the $\mathrm{Z}_{\text {total }}$ and $\mathrm{V}_{\text {source }}$ axes. Nevertheless a ridge can be seen going to lower total detected charge as the source velocity decreases. A possible explanation for this dependence will be presented in part b of this section. Furthermore, in these plots a high density of contour lines is observed centered around the point $\left(\mathrm{Z}_{\text {total }}=79, \mathrm{~V}_{\text {source }}\right.$ $=10.6 \mathrm{~cm} / \mathrm{ns}$ ). The high source velocity of these events suggests that they arise from peripheral reactions, whereas their total charge (around $\mathrm{Z}=79$ ) indicates that they originate from the fission of the Au-like nucleus.

The top arrow shown in figures 5.9b-e (upper left quadrant) indicates the value of the beam velocity $V_{\text {beam }}$ while the bottom arrow corresponds to the source velocity $V_{c f}$ 
value arising from complete fusion. The events below the complete fusion velocity have no physical meaning, and can be characterized as incomplete coincidence events, in which one or more fragments were not detected. However, incomplete events can occur for velocities larger than $V_{c f}$ as well. One way of reducing the number of incomplete events is to consider events only above a certain Ztotal value. This method has been utilized in section 5.2.3

\section{b) Possible Interpretation}

The distribution of events in the $\mathrm{V}_{\text {source }}-\mathrm{Z}_{\text {total }}$ plane, for the heavier targets (Figures $5.9 \mathrm{~b}-\mathrm{e})$, is characterized by a ridge that gradually goes to lower $Z_{\text {total }}$ values as the source velocity decreases. The dependence shown by the data in the $\mathrm{V}_{\text {source }}-\mathrm{Z}_{\text {total }}$ plane is opposite to what is observed at lower incident energies. For instance, in the 18 $\mathrm{MeV} / \mathrm{A} \mathrm{La}+\mathrm{Cu} / \mathrm{Ni}$ reactions [Cha 90, Col 89] a ridge was seen going to lower total charge as a function of increasing source velocity. In both types of reactions (18 $\mathrm{MeV} / \mathrm{A} \mathrm{La}+\mathrm{Cu} / \mathrm{Ni}$ and $60 \mathrm{MeV} \mathrm{Au}$-induced reactions) the pattern of the data can be explained by the range of incomplete fusion processes that is present.

For the $18 \mathrm{MeV} / \mathrm{A} \mathrm{La}+\mathrm{Cu} / \mathrm{Ni}$ the explanation for the observed pattem is clear: in reverse kinematics, the composite system formed in incomplete fusion processes is characterized by lower velocities as the projectile picks up more mass from the target. Furthermore light particle evaporation over the entire range of incomplete fusion processes is small and does not strongly effect the expected dependence of the ridge. At higher incident energies (like $60 \mathrm{MeV} / \mathrm{A}$ ) however, the evaporation process may become more extensive and may substantially alter the pattern of the data observed at lower energies. An explanation[Rou 93] of how the pattern may change because of extensive evaporation is given below. 
Figure 5.10, taken from [Rou 93], illustrates schematically the competing role of incomplete fusion and charged particle evaporation. The thick line represents, for a particular system, the range of primary products resulting from the incomplete fusion process before evaporation. This line should be the same at all bombarding energies. The dashed lines to the left represent the range of products resulting from incomplete fusion after evaporation for the same system, but for different bombarding energies. Assuming that the evaporation process does not substantially alter the source velocity, the dashed line should rotate towards the left as the bombarding energy is increased, since the maximum excitation energy is always for complete fusion. The bombarding energy for which all of the charge gained in the incomplete fusion process is equal to the charge lost by evaporation is represented by the vertical dashed line. Above this line more than one charge unit is lost on the average by evaporation for each charge unit transferred from the target to the projectile. Therefore, for excitation energies associated with extensive evaporation, the distribution of events in the $Z_{\text {total }}-V_{\text {source }}$ plane is expected to shift towards lower total charge as the source velocity decreases. As shown in Figures $5.9 \mathrm{~b}-\mathrm{e}$ this is the pattern displayed by the $60 \mathrm{MeV} / \mathrm{A} \mathrm{Au}+\mathrm{Al}, \mathrm{V}$, $\mathrm{Cu}$ and Au data. A similar result has also been reported in reference [Rou 93]. Roussel et al. [Rou 93] found that in the La-induced reactions the vertical dashed line corresponds to approximately $31 \mathrm{MeV} / \mathrm{A}$.

\subsection{3 $\mathrm{Z}$ and $\mathrm{V}$ distributions according to fragment multiplicity.}

\section{a) Z - distributions}

A more instructive overview of the reaction mechanism in the $\mathrm{Au}+\mathrm{C}, \mathrm{Al}, \mathrm{V}, \mathrm{Cu}$, and $\mathrm{Au}$ reactions can be obtained by sorting the events according to their multiplicity. 
Figure 5.11 shows the $Z_{\text {total }}$ distributions for the 2 -fold, 3-fold, 4 -fold and 5-fold events. For the 2 -fold events, and for all targets, a narrow peak around $\mathrm{Z}=79$ is observed, which can be associated with the binary fission of Au-like projectiles. At the same time, for the heavier targets ( $\mathrm{Al}, \mathrm{V}, \mathrm{Cu}$ and $\mathrm{Au}$ ), an approximately flat tail of events can be seen extending to low $\mathrm{Z}$ values. This tail may arise from higher $\mathrm{n}$-fold events in which only two fragments were detected and one or more fragments were missed. The magnitude of these tails rises gradually for progressively heavier targets with a dramatic increase in the case of the $60 \mathrm{MeV} / \mathrm{A} \mathrm{Au}+\mathrm{Au}$ reactions. This effect can be associated with the progressively larger range of excitation energies available in the center-of-mass for the heavier targets (especially for the Au target), since the multibody decay probability is predicted (see Chapter 2 ) to increase as a function of increasing excitation energy.

On the other hand, the spectra of the higher-fold $(3,4$, and 5 -fold) events are dominated by a single broad distribution. This distribution shifts to greater values of $\mathrm{Z}_{\text {total }}$ with increasing multiplicity and is consistent with a larger fraction of the total charge being detected. Furthermore, the $Z_{\text {total }}$ distributions sharpen up for progressively higher multiplicities for all targets, indicating that the detected higher n-fold events, i.e. 5 -fold, may be more complete.

\section{b) V - distributions}

As was discussed in section 5.2.2 a substantial number of the coincidence events may include incomplete events in which one or more fragments were missed. In order to reduce the contamination arising from incompletely detected events, the source velocity distribution were constructed only from events with a total measured charge larger than $Z_{\text {total }}=35$. Such an approach should reduce some of the bias in the Vs distributions that would otherwise be included by the incorrect kinematic reconstruction of the incomplete events. 
The normalized source velocity distributions obtained at $60 \mathrm{MeV} / \mathrm{A}$ for all the targets and for the different fragment multiplicities are presented in Figure 5.12. The vertical dashed line in each subplot indicates the beam velocity. The source velocity distributions broaden considerably as the mass of the target increases. This can be clearly seen by comparing the source velocities of the 2- and 3-fold events from the carbon and $\mathrm{Au}$ targets. Within the incomplete fusion model, the increased width can be attributed to a larger range of impact parameters that gives rise to a larger range of incomplete fusion products. Light particle evaporation (l.p.e) also contributes to the broadening of the source velocity distribution. The amount of broadening due to l.p.e. has been estimated at $60 \mathrm{MeV} / \mathrm{A}$ with the statistical code GEMINI [Ch 88a-b]. Calculations show that for the ${ }^{12} \mathrm{C}$ target, the width can be explained almost entirely by light particle evaporation, whereas, for the heavier targets such as $\mathrm{Al}$, evaporation accounts for only about a third of the observed width. Thus, for the heavier targets, the width of the source velocity distribution can be effectively associated with a range of incomplete fusion processes.

For the higher $n$-fold events $(n=3,4,5)$ the average source velocity progressively shifts to lower values as a function of increasing multiplicity. This can be seen more readily by examining Table 5.1 , that lists the average source velocity values of the 2 -, 3-, 4-, and 5-fold events for all targets. Within the framework of the incomplete fusion model, such a dependence can be attributed to a larger mass transfer from the target to the projectile at progressively smaller impact parameters, since a larger mass transfer slows down the source to a larger degree. Therefore, the higher multiplicity events may arise from sources with higher excitation energies that were formed in more central collisions. A similar result has also been reported in reference [Rou 93]. 


\begin{tabular}{rccccc} 
& $\mathrm{C}$ & $\mathrm{Al}$ & $\mathrm{V}$ & $\mathrm{Cu}$ & $\mathrm{Au}$ \\
2-fold & 10.50 & 10.0 & 10.0 & 10.0 & 9.9 \\
3-fold & 10.32 & 9.4 & 9.4 & 9.4 & 9.3 \\
4-fold & - & 9.2 & 9.2 & 9.1 & 9.0 \\
5-fold & - & 9.0 & 9.0 & 9.0 & 8.9 \\
\hline
\end{tabular}

Table 5.1 Average source velocities in $\mathrm{cm} / \mathrm{ns}$.

\subsection{4 $\mathrm{Z}_{1}-\mathrm{Z}_{2}$ Plots}

For the two-body events the atomic number $Z_{1}$ of one fragment was plotted against the atomic number $\mathrm{Z}_{2}$ of the second. This method of plotting the data, can be useful in determining whether the decay mechanism is predominantly binary or multibody. If the decay mechanism is binary, the contour plots should be dominated by a band of events that peaks along the $Z_{1}+Z_{2} \approx Z_{\text {source }}$ line. In addition the band should broaden and shift towards smaller total charge as the excitation energy increases, because of evaporation. However, if the final state is actually multibody with one or several fragments not detected, the events should fall below the line.

The measured $Z_{1}-Z_{2}$ correlations for all systems studied are shown in Figure 5.13. The diagonal dashed lines in these figures indicate the charge of the Au projectile $\left(Z_{1}+\right.$ 
$Z_{2}=79$ ). The pattern observed for the $A u+C$ reaction is quite clear. For this very asymmetric system, the contour lines show a distinct band parallel to the dashed line, thus illustrating the binary nature of the process. The maximum in this plot occurs around $\left(Z_{1}=40, Z_{2}=40\right)$ and corresponds to the symmetric fission of the Au-like projectiles.

A well-defined band of events running diagonally along the line $Z_{1}+Z_{2}=79$ (dashed line), can also be seen for the heavier targets ( $\mathrm{Al}, \mathrm{V}, \mathrm{Cu}$, and $\mathrm{Au}$ ). The contour lines comprising the band indicate that there is a high concentration of events near symmetry. These events, as will be shown in section 5.2.7, can be associated with source velocities corresponding to peripheral reactions that lead predominantly to the binary fission of the Au-like projectiles. In addition, a substantial number of events appears at low $Z_{1}$ and $Z_{2}$ values. These events suggest that a large fraction of the binary events may in fact be multibody events in which only two of the fragments were detected.

To determine whether the observed distribution of events along the band in the $Z_{1}$ $\mathrm{Z}_{2}$ plane was biased by the detection efficiency we relied on Monte Carlo simulations. The average efficiency for detecting the nearly symmetric binary decays $Z_{1}+Z_{2}=$ $(39+40,38+41,37+42)$ was estimated to be about $55 \%$ while that for detecting the asymmetric decays $\mathrm{Z}_{1}+\mathrm{Z}_{2}=(21+58,22+57,23+56)$ was around $46 \%$. Such a difference in the efficiencies may not strongly influence the observed distribution of events (along the band) in the $Z_{1}-Z_{2}$ plots.

\subsubsection{Excitation Functions}


To study the behavior of hot nuclear systems as their excitation energy increases, "excitation functions" for the multifold events were constructed from the sourcevelocity distributions. The relative abundance of binary, ternary, quaternary and quinary events was determined for different bins of the source velocity, and therefore also of the corresponding excitation energy of the source. In Figure 5.14 the excitation functions for the multifold events obtained from the source velocity distributions are plotted as a function of the excitation energy per nucleon $Q$ of the source. $P(n)$ represents the proportion of $n$-fold events with respect to the total number of coincidence events: $P(n)=\frac{N(n)}{N(2)+N(3)+N(4)+N(5)}$, where $N(n)$ is the number of $\mathrm{n}$-fold events. Evaporation residues, corresponding to $\mathrm{n}=1$, were not considered, since in reverse kinematics they are confined to a very small angle around the beam direction, where the detection efficiency is small. Furthermore, in order to reduce the contamination arising from incompletely detected events, only events with total measured charge larger than 35 were considered.

The excitation energy per nucleon $Q$ was determined, within the incomplete-fusion model, from the following equation :

$$
Q=\left(\frac{E}{A}\right)_{\text {beam }} \times \frac{V_{\|}}{V_{\text {beam }}} \times\left(1-\frac{V_{\|}}{V_{\text {beam }}}\right),
$$

where $\mathrm{E} / \mathrm{A}(=60 \mathrm{MeV} / \mathrm{A})$ is the bombarding energy per nucleon, $\mathrm{V}_{\|}$is the parallel source velocity and $V_{\text {beam }}$ is the beam velocity. (Equation 5.4 was derived from equation F.2, see Footnote 1). Because such a model does not take into account preequilibrium particle emission processes, the quantity $\mathrm{Q}$ may be considered as an upper limit of the actual excitation energy. The uncertainty on the horizontal scale associated with the emission of preequilibrium nucleons was estimated with the dynamical code described in Chapter 6 and was found to be at most around $45 \%$ for the 
systems considered in this study. Furthermore, since it is not clear how accurately this code simulates the preequilibrium emission at bombarding energies of $60 \mathrm{MeV} / \mathrm{A}$, the estimated uncertainty may be characterized by a large error. Nevertheless, the uncertainty associated with $Q$ should only overpredict the excitation energy of the decaying system and may not strongly influence the simple pattern observed in the data. notwithstanding

The probabilities for 3, 4, and 5-fold increase substantially as a function of $Q$ over a large range of excitation energies (see Figure 5.14). Such a dependence suggests that there may be a strong relationship between the excitation energy and the source velocity, since the probability for multifragment decay is predicted (see Chapter 2) to increase as a function of increasing excitation energy. In addition, the sharp rise of the branching ratios indicates that the width of the source velocity distributions may arise primarily from the range of sources formed in incomplete fusion processes and may be only partly due to evaporation. If light particle evaporation was the dominant source of broadening, the excitation functions would be expected to be flat.

Furthermore the excitation functions increase smoothly up to approximately 7-9 $\mathrm{MeV} / \mathrm{A}$ without showing any discontinuity. In contrast, several statistical multifragmentation calculations [Bo $83, \mathrm{Gr} 87$ ] predict a sudden rise in the multibody probability at an excitation energy between $3 \mathrm{MeV} / \mathrm{A}$ and $5 \mathrm{MeV} / \mathrm{A}$.

An extraordinary feature (in Figure 5.14) is that the multifold probabilities for all of the targets, with the exception of carbon, are almost identical. This behavior suggests that the competition between the various multifragment channels is independent of the entrance channel. The similarity between the excitation functions for the different targets indicates that the sources produced in these reactions depend relatively little on the actual nature of the target and may be characterized primarily from the amount of mass transferred from the target to the projectile. A similar result has also been 
observed for the La induced reactions in reference [Rou 92]. In addition the authors of [Rou 93] found that the excitation functions were almost independent of the bombarding energy as well. Thus it appears that once the excitation energy is determined from the source velocity, the resulting branching ratios for the various multifragment channels are fixed, suggesting that statistics may play an important role in multifragmentation.

In the case of the carbon target however, the multifold probabilities are systematically lower and flatter than those for the heavier targets. One possible explanation for this difference may be that the broadening of the source velocity (excitation energy) bins is due to extensive light-particle evaporation. As already mentioned, calculations show that for the ${ }^{12} \mathrm{C}$ target, the width of the source velocity can be explained almost entirely by light particle evaporation, whereas, for the heavier targets evaporation accounts for only about a third of the observed width (the rest arising from incomplete fusion processes).

\subsubsection{Efficiency Corrected Excitation Functions}

Since the coincidence data have not been corrected for the efficiency of the detector, it was necessary to verify that the observed excitation functions were not strongly biased by some experimental artifact. To this end we simulated the reaction $\mathrm{Au}+\mathrm{Cu}$ at $60 \mathrm{MeV} / \mathrm{A}$ following the procedure described in Chapter 6 (LV + GEMINI calculations). Sets of $2,3,4$, and 5-fold events were generated, whose charge and velocity distributions are presented in Chapter 6. The simulated events were then filtered through a software replica of our detector in order to estimate the contamination 
due to incompletely detected events. Detector thresholds were included in the filter to account for high velocity fragments punching through the $5 \mathrm{~mm} \mathrm{Si}$.

$$
\begin{aligned}
& e_{5}^{5}=17 \% \quad e_{5}^{4}=39 \% \quad e_{5}^{3}=27 \% \quad e_{5}^{2}=11 \% \\
& \mathrm{e}_{4}^{4}=23 \% \quad \mathrm{e}_{4}^{3}=40 \% \quad \mathrm{e}_{4}^{2}=28 \% \\
& \mathrm{e}_{3}^{3}=31 \% \quad \mathrm{e}_{3}^{2}=45 \% \\
& \mathrm{e}_{2}^{2}=50 \%
\end{aligned}
$$

Table 5.2 Detection efficiency factors as determined from reaction simulations.

The efficiencies for the 2-, 3-, 4-, and 5-fold events as determined from these simulations are listed in Table 5.2. In this table $\underset{\mathrm{g}}{\mathrm{i}}$ represents the efficiency of a j-fold event being detected as an $\mathrm{i}$-fold event, as determined from the simulations. These efficiency factors were then used along with the experimentally determined number $n_{j}$ of $\mathrm{j}$-fold events to calculate the true number $\mathrm{N}_{\mathrm{j}}$ of $\mathrm{j}$-fold events. For example, the true number $\mathrm{N}_{2}$ of 2-body events was determined by subtracting from $\mathrm{n}_{2}$ the various contributions of the 3-,4-, and 5-fold events. The equations used for the corrections are the following:

$$
n_{5}=N_{5} * e_{5}^{5}
$$




$$
\begin{aligned}
& \mathrm{n}_{4}=N_{4} * e_{4}^{4}+N_{5} * e_{5}^{4} \\
& n_{3}=N_{3} * e_{3}^{3}+N_{4} * e_{4}^{3}+N_{5} * e_{5}^{3} \\
& n_{2}=N_{2} * e_{2}^{2}+N_{3} * e_{3}^{2}+N_{4} * e_{4}^{2}+N_{5} * e_{5}^{2} .
\end{aligned}
$$

The uncertainty in the true number $\mathrm{N}_{\mathrm{j}}$ of events has been calculated by utilizing the statistical error associated with the detected number $n_{j}$ of events. Tables 5.3 and 5.4 show the errors associated with the numbers $n_{j}$ and $N_{j}$ as a percentage of $n_{j}$ and $N_{j}$ respectively. By examining these tables it can be seen that the uncertainty in $\mathrm{N}_{5}$ is equal to the error in $\mathrm{n}_{5}$; this is because $\mathrm{N}_{5}$ was determined from $\mathrm{n}_{5}$ by a simple multiplication operation. The uncertainty in $\mathrm{N}_{\mathrm{j}}$, for the 3 and 4 -fold events, can be larger by a factor of two from the corresponding statistical error in $\mathrm{n}_{\mathrm{j}}$; nontheless the errors associated with $\mathrm{N}_{\mathrm{j}}$ are still relatively small. The large uncertainties associated with $\mathrm{N}_{4}$ at 1 and $2 \mathrm{MeV} / \mathrm{A}$ are due to the corresponding large statistical errors in $\mathrm{n}_{4}$. Even the value of the error associated with the true number $\mathrm{N}_{2}$ is relatively small over a large range of excitation energies $\mathrm{E}^{*}$ (1-5 MeV/A). A significant difference between the values of the errors associated with $n_{2}$ and $N_{2}$ is observed only for the 6 and 7 $\mathrm{MeV} / \mathrm{A}$ excitation energies. Therefore the overall errors introduced in $\mathrm{N}_{\mathrm{j}}$ suggest that this procedure is not a significant source of error.

The corrected multifold probabilities for the $\mathrm{Au}+\mathrm{Cu}$ reactions are shown in Figure 5.15. Similar corrections have also been applied for the $\mathrm{Au}+\mathrm{Al}, \mathrm{V}$, and $\mathrm{Au}$ reactions and will be presented in section 6.3. The corrected probabilities for the 2 -fold events decrease dramatically as a function of increasing excitation energy and disappear around $8 \mathrm{MeV} / \mathrm{A}$. This dependence is very different from that displayed by the uncorrected 2-fold probabilities and suggests that at high energies (i.e. larger than 8 $\mathrm{MeV} / \mathrm{A}$ ) the observed binary events may be actually multibody events in which one or more fragments were missed. 
On the other hand the corrected probabilities, for the 3-, 4-, and 5-fold events, increase smoothly as a function of excitation energy similarly to the uncorrected probabilities. This result indicates that the smooth sharp rise of the excitation functions, for the 3, 4, and 5-fold events, is real and the data is not be biased in any significant way by our detector efficiency.

\begin{tabular}{llllll}
$E^{*}(\mathrm{MeV} / \mathrm{A})$ & 2-fold & 3-fold & 4-fold & 5-fold \\
\hline 1 & & & & \\
\hline 2 & $.54 \%$ & $2.8 \%$ & $16.0 \%$ & $75.0 \%$ \\
3 & $.56 \%$ & $1.8 \%$ & 8.2 & $\%$ & $40.4 \%$ \\
4 & $.62 \%$ & $1.4 \%$ & 4.9 & $\%$ & $20.7 \%$ \\
5 & $.68 \%$ & $1.2 \%$ & 3.4 & $\%$ & $16.9 \%$ \\
6 & $.79 \%$ & $1.1 \%$ & 2.8 & $\%$ & $10.1 \%$ \\
7 & $1.0 \%$ & $1.2 \%$ & 2.8 & $\%$ & $8.5 \%$ \\
& $1.2 \%$ & $1.3 \%$ & 2.7 & $\%$ & $7.7 \%$ \\
\hline
\end{tabular}

Table 5.3 Statistical error in the detected number $n_{j}$ of $j$-fold events as a percentage of $n_{j}$. 


\begin{tabular}{ccccc} 
E* (MeV/A) & 2-fold & 3-fold & 4-fold & 5-fold \\
\hline 1 & & & & \\
\hline 2 & $.60 \%$ & $3.2 \%$ & $20.0 \%$ & $75.0 \%$ \\
3 & $.75 \%$ & $2.2 \%$ & $10.7 \%$ & $40.4 \%$ \\
4 & $1.1 \%$ & $1.9 \%$ & $6.2 \%$ & $20.7 \%$ \\
5 & $1.9 \%$ & $2.0 \%$ & $5.0 \%$ & $16.9 \%$ \\
7 & $4.1 \%$ & $2.4 \%$ & $4.7 \%$ & $10.1 \%$ \\
7 & $12.1 \%$ & $3.1 \%$ & $4.8 \%$ & $8.5 \%$ \\
\hline
\end{tabular}

Table 5.4 Uncertainty in the true number $\mathrm{N}_{\mathrm{j}}$ of $\mathrm{j}$-fold events as a percentage of $\mathrm{N}_{\mathrm{j}}$.

\subsubsection{Fragment distributions gated by source velocity}

The size distribution of the fragments produced in a collision may depend on the excitation energy of the source from which they are emitted. This dependence has been examined by studying the evolution of the charge distribution as a function of the source velocity Vs, since as was shown in section 5.2 .5 , the source velocity can be a good measure of the excitation energy. In order to reduce contamination arising from incomplete events, only events with total measured charge larger than 35 were considered.

The charge distributions for different gates of the source velocity are shown in Figures 5.16a-b, for the $\mathrm{C}$ and $\mathrm{Cu}$ targets. The fragment yields from the $\mathrm{V}, \mathrm{Al}$ and $\mathrm{Au}$ 
targets (not shown) are very similar to the ones from the $\mathrm{Cu}$ target and therefore the discussion presented in this section for the $\mathrm{Cu}$ target applies to them as well. The number written in each frame (see Figure 5.16) is the excitation energy per nucleon of the emitting source. This value was calculated from the average source velocity of the gates, within the incomplete fusion model, by means of eqn. 5.4.

In the case of the $C$ target there is no significant change in the charge distribution for the different gates. All frames are characterized by a peak around $Z=40$ arising from the fission of the Au-like projectiles. It appears that the binary decay of the Aulike projectiles is favored over other decay channels, such as multifragmentation. This behavior can be attributed to the small size of the carbon target that limits the available excitation energy. As was discussed in Chapter 1, the maximum excitation energy per nucleon available in the center-of-mass for the $\mathrm{Au}+\mathrm{C}$ system is around $\mathrm{E}^{*} / \mathrm{A} \sim 3 \mathrm{MeV}$, while multifragmentation is generally predicted to occur in the range of about $E^{*} / A \sim 4$ $\mathrm{MeV}$ [Gr 85, Bon 85].

On the other hand, the larger size of the $\mathrm{Cu}$ target offers a larger range of impact parameters, and several trends are observed with increasing excitation energy. At the lowest excitation energy $(1 \mathrm{MeV} / \mathrm{A})$ the picture is dominated by a single peak centered around $Z=40$. These events arise mainly from the fission of Au-like projectiles produced in peripheral collisions. In addition a modest yield of light fragments is also observed.

At higher excitation energies, namely between 2 and $6 \mathrm{MeV} / \mathrm{A}$, the charge distributions change noticeably. The fission peak is gradually replaced by a hump, and the yield of light particles progressively becomes larger. The change in the shape of these distributions can be attributed to the growing importance of other modes of decay, such as multifragmentation, relative to binary fission. 
At still higher excitation energies, E $>6 \mathrm{MeV} / \mathrm{A}$, the fission peak disappears completely. The distributions are now characterized by a large yield of light fragments. Furthermore the distributions progressivelly withdraw to smaller values of $Z$ as the excitation energy increases. This dependence suggests that the exit channels (such as multifragmentation) associated with the highest energies and possibly with the most central collisions lead dominantly to the production of small fragments.

In summary, for the heavier targets, strong correlations are observed between the source velocity and the size of the fragments emitted in a reaction. On the one hand, high source velocity gates select predominantly fragments around $\mathrm{Z} 40$ produced in the decay of Au-like nuclei generated in peripheral collisions; on the other hand, low source velocity gates select predominantly light fragments that are emitted from the multifragmentation of a source formed in central collisions. Thus it appears that the source velocity can provide a good measure of the impact parameter.

\subsubsection{Relative abundance of fragments as a function of excitation energy.}

Interesting results regarding the production of complex fragments can be obtained by examining the evolution of the relative abundance of the fragments as a function of the excitation energy. This is just another way of plotting the data presented in Figure 5.16 .

The relative abundance of fragments with atomic number $\mathrm{Z}=\mathrm{i}$ was determined from the ratio $N_{i} /\left(N_{4}+N_{5}+\ldots+N_{n}\right)$ for different bins of the source velocity, where $N_{i}$ is the total number of fragments with $\mathrm{Z}=\mathrm{i}$ within a particular bin. The excitation energy was calculated from the average source velocity of each bin by means of eqn. 5.4. In order 
to reduce contamination arising from incomplete events, only events with total measured charge larger than 35 were considered.

In Figure 5.17 the relative yields as a function of the excitation energy are presented for the fragments (with $\mathrm{Z}$ between $10<\mathrm{Z}<64$ ) produced in the $60 \mathrm{MeV} / \mathrm{A} \mathrm{Au}+\mathrm{Cu}$ reactions. Similar trends have also been observed in the relative yields from the $\mathrm{V}, \mathrm{Al}$, and $\mathrm{Au}$ targets; even for the carbon the trends are very similar to the $\mathrm{Cu}$ target. Therefore the discussion presented in this section applies to all targets.

The relative yield of the lighter fragments $(10<\mathrm{Z}<22)$ increases with increasing excitation energy. This dependence indicates that the mechanisms responsible for the production of light fragments, such as multifragmentation, become gradually more important at larger excitation energies.

For heavier fragments $(22<Z<52)$, however, the relative yields undergo a dramatic change as a function of $\mathrm{Z}$. While the yields associated with the fragments between $22<Z<32$ are relatively flat over the entire range of excitation energies, on the other hand the relative yields for fragments between $32<\mathrm{Z}<52$ decrease as a function of increasing energy. For fragments with $Z \sim 40$ the relative yields decrease by nearly one and a half orders of magnitude. This behavior can be attributed to the fission of the Au-like projectiles which is the dominant mode of complex fragment production at low excitation energies and becomes gradually less important at higher excitation energies.

Finally, Figure 5.17 indicates that the relative production of fragments with atomic number $Z$ between $54<Z<64$ (and therefore also the corresponding decay mechanism ) is favored at intermediate excitation energies. These fragments can arise from the asymmetric decay or extensive evaporation of sources that were formed in incomplete fusion reactions at intermediate impact parameters. 


\subsection{COINCIDENCE DATA WITH THE NEUTRON DETECTOR}

\subsubsection{Neutron Multiplicity Distributions}

As was discussed in Chapters 3 and 4, the number of neutrons emitted in the 60 $\mathrm{MeV} \mathrm{Au-induced} \mathrm{reactions} \mathrm{was} \mathrm{measured} \mathrm{by} \mathrm{utilizing} \mathrm{a} \mathrm{novel} \mathrm{neutron} \mathrm{calorimetric}$ approach [ $\mathrm{Pa} 88, \mathrm{~Pa} 90]$ which is particularly suitable for the measurement of highenergy neutrons. Figure 5.18 show's the measured neutron multiplicity distributions for the $60 \mathrm{MeV} / \mathrm{A}{ }^{197} \mathrm{Au}+{ }^{27} \mathrm{Al},{ }^{51} \mathrm{~V}$, nat $\mathrm{Cu}$, and ${ }^{197} \mathrm{Au}$ reactions. These distributions were obtained by initiating the neutron counting or light collection whenever the Si-array was triggered by at least one fragment. Consequently, the distributions of Figure 5.18 represent the full spectrum of detected processes in the Si-array.

A dominant feature in these plots is that the probability of events decreases as a function of increasing multiplicity for all reactions. The neutron distributions span nearly four orders of magnitude and, as will be shown in the following sections, they can be correlated with the excitation energy. Low multiplicities are associated with peripheral, weakly damped collisions that are characterized by small excitation energies; on the other hand the highest neutron multiplicities result from the more central collisions and are characterized by high excitation energies.

Furthermore the distributions extend progressively to larger values of the neutron multiplicity as the mass of the target increases. This may be an indication that higher excitation energies are attained for increasingly symmetric projectile-target combinations. 


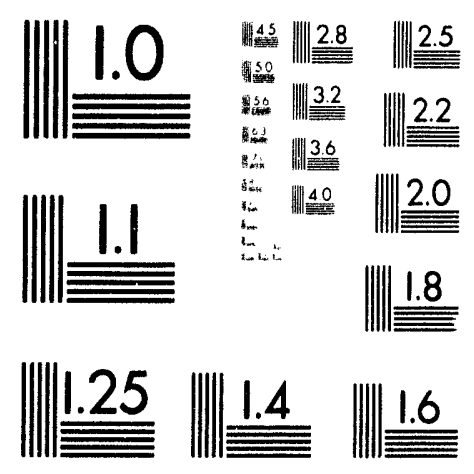



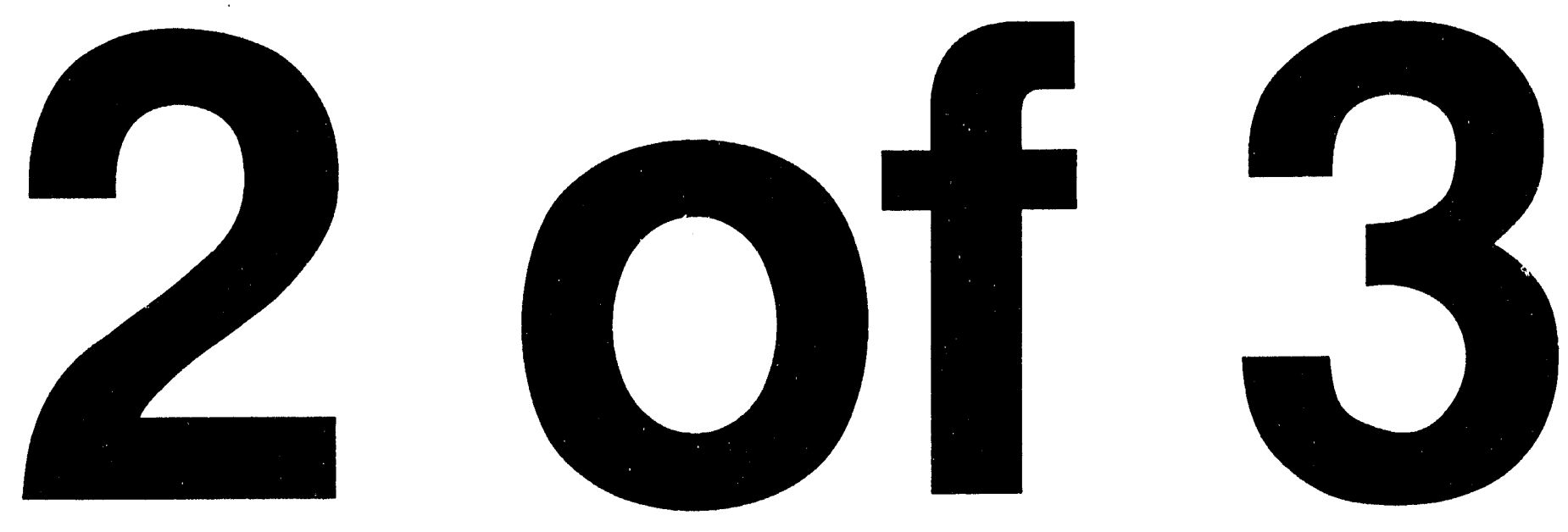
The neutron multiplicity distributions presented in Figure 5.18 are quite different from those observed in other studies. As an example the neutron multiplicity spectrum for the ${ }^{208} \mathrm{~Pb}+{ }^{197} \mathrm{Au}$ at $29 \mathrm{MeV} / \mathrm{A}$ measured [Pi 91] with a Gd-loaded liquid scintillator is shown in Figure 5.19. In this figure the high yield of events occuring at low multiplicities has been associated with peripheral reactions, while the broad bump at higher multiplicities has been attributed to the more central collisions. The reasons for such a difference between the neutron multiplicity spectra measured in this study and those of Figure 5.18 are not clear.

However, it is important to remember that the calibrations of the neutron calorimeter are model dependent and therefore the distributions shown in Figure 5.18 may not reflect the true neutron multiplicities. Furthermore, as discussed in Chapter 4, a scaling factor was used to normalize the observed light output to the calculated neutron multiplicities; this may be a source of additional error. Nevertheless, such errors and problems may only affect the neutron multiplicity scale and should not influence the simple trends observed in the data presented in the following sections.

\subsubsection{Fragment distributions gated by neutron multiplicities.}

In section 5.2.7 the change in the charge distribution of the emitted fraginents as a function of the source velocity was examined, and a relation between the source velocity and the impact parameter was suggested. In order to determine if a similar correlation between the neutron multiplicity and the impact parameter can be established, we have studied the evolution of the fragment charge distribution as a function of the number of neutrons emitted. 
Figure 5.20 shows exclusive fragment yields obtained for five neutron multiplicity $\mathrm{Mn}$ gates, in the $60 \mathrm{MeV} / \mathrm{A} \mathrm{Au}+\mathrm{Cu}$ reactions. The fragment yields from the $\mathrm{V}, \mathrm{Al}$ and Au targets (not shown) are very similar to those from the $\mathrm{Cu}$ target and therefore the discussion presented in this section for the $\mathrm{Cu}$ target applies to them as well. The range of the neutron multiplicity gates is written in each frame.

In the frame associated with the lowest multiplicity (GATE 1), the charge distribution is characterized by a broad peak centered around $Z=39$ which can be associated with the fission of the Au-like projectiles produced in peripheral reactions. Furthermore the distribution extends over a large range of $Z$ values; in the case of the Au target a peak is observed at $Z=79$ that can be attributed to the elastically scattered Au-projectiles produced at large impact parameters.

In GATE 2 the charge distribution changes considerably as the fission fragment peak is replaced by a bump. This means that the fission of the Au-like projectiles is becoming less important and reactions occurring at smaller impact parameters are more likely. In addition the tail of the charge distributions recedes to lower values of $Z$.

For higher neutron multiplicities (GATEs 3,4,5) the distributions are characterized by a high yield of light fragments; the distributions decrease almost exponeritially with increasing atomic number. This indicates that the production of light mass fragments becomes the dominant decay channel. Furthermore the tail of the charge distributions gradually recedes to lower values of $\mathrm{Z}$ with increasing neutron multiplicity. The correlation between the high neutron multiplicities and the low probability for heavier fragments suggests that, in central collisions, the $\mathrm{Au}+\mathrm{Al}, \mathrm{V}, \mathrm{Cu}, \mathrm{Au}$ systems may disintegrate preferentially into a large number of nucleons and small fragments.

The fragment distributions gated by neutron multiplicity (see Figure 5.20) have a qualitative similarity with the fragment distributions gated by source velocity (see Figure 5.16b). For instance a peak is observed in the distributions around $Z \sim 40$ for 
both low excitation $E^{*}$ energies (high source velocities Vs) and low neutron multiplicities $\mathrm{Mn}$. This peak disappears in both types of distributions for higher $\mathrm{E}^{*}$ and Mn. Furthermore the distributions gradually recede to lower atomic number values for progressivelly larger $\mathrm{E}^{*}$ and $\mathrm{Mn}$ values. Thus low neutron multiplicities can be associated with low excitation energies while high neutron multiplicities arise from the more central collisions and are characterized by high excitation energies.

\subsubsection{Excitation Functions}

In section 5.2 .5 excitation functions for the $n$-fold $(n=2,3,4$, and 5$)$ events were constructed as a furction of the excitation energy per nucleon of the source. The excitation energy was determined, within the incomplete fusion model, from the source velocity Vs by means of eqn. 5.4. However, it would be useful to have an independent estimate of the deposited excitation energy $E^{*}$, since $E^{*}$ cannot be extracted precisely from the source velocity. In this section, excitation functions simiiar to those of section 5.2.5 were constructed by gating on the neutron multiplicity Mn distribution, since the number of neutrons emitted in a reaction can provide a good measure for the excitation energy of the source.

In Figure 5.21 the "excitation functions" for the multifold events obtained from the neutron multiplicity distributions are plotted as a function of $\mathrm{Mn}$. The probabilities for the three, four, and five-fold events increase as a function of neutron multiplicity $\mathrm{Mn}$, indicating, that $\mathrm{fcr}$ progressively larger neutron multiplicities, multibody decay becomes an increasingly important exit channel.

A remarkable feature is that for all targets, these excitation functions span approximately the same order of magnitude in $P(n)$. This suggests that the competition 
between the various multifragment channels is independent of the entrance channel and depend relatively little on the actual nature of the target. A similar behavior was also observed for the excitation functions presented in section 5.2.5.

Furthermore, the rate of multifold events increases smoothly with increasing neutron multiplicity without showing any discontinuity. This should be contrasted with several statistical multifragmentation calculations [Bo 83, Gr 87] which predict a sudden rise in the multibody probability.

Finally, although these excitation functions do not exhibit the same steep rise as those of Fig 5.14, they do reproduce quite well the trend and magnitude of that part of the $P(n)$ functions of Figure 5.14 greater than $E / A>3 \mathrm{MeV} / \mathrm{A}$. The reason for this difference is not clear.

\subsubsection{Excitation Energy vs Neutron Multiplicity}

In this experiment the simultaneous availability of the neutron multiplicity and of the source velocity provided the opportunity to examine directly the relationship between the excitation energy of a hot source formed in a particular reaction and the number of neutrons emitted in that reaction.

Figure 5.22 displays the dependence of the excitation energy $E^{*}$ upon the neutron multiplicity $\mathrm{Mn}$ for the heavier targets ( $\mathrm{Al}, \mathrm{V}, \mathrm{Cu}$, and $\mathrm{Au}$ ). The excitation energy $\mathrm{E}^{*}$ was determined from the source velocity by means of eqn. F.2. The distribution of the events in the $E^{*}$ vs Mn plane is very broad and it is difficult to discern any relationship between the two variables. In order to determine if a correlation exists between $E^{*}$ and Mn we have plotted in Figure 5.23, the average neutron multiplicity versus the 
excitation energy, for the heavier targets. When plotted in this way, the data display an interesting dependence.

For all targets (see Figure 5.23) the average neutron multiplicity increases initially as a function of energy, it reaches a saturation value and remains approximately constant with increasing excitation energy. This behavior can be attributed to charged particle emission that becomes more important with increasing excitation energy. For instance, it is possible that for weakly excited heavy nuclei characterized by low neutron multiplicities, the energy is essentially removed by neutrons. This is due to their low binding energy and the absence of a Coulomb barrier. It is only when sufficient amount of excitation energy has been stored in the system that light charged particle (l.c.p) emission sets in. In reference [Sch 91] the emission of light charged particles was determined to set in after the emission of a minimum number of neutrons called the neutron multiplicity threshold. Once the threshold had been reached the l.c.p multiplicity increased roughly linearly with the measured neutron multiplicity.

A similar dependence of the neutron multiplicity with increasing excitation energy has also been observed by Knoche et. al. [Kno 92]. Figure 5.24 taken from reference [Kno 92] shows the neutron multiplicities for several reaction systems in the fissility range $x=0.82-0.91$ as a function of the excitation energy. For all reactions the neutron multiplicities increase slowly up to some saturation value. Knoche et. al. determined that the neutron multiplicity was not an accurate measure of $\mathrm{E}^{*}$ at higher excitation energies because of the increasing competition of charged particle emission.

However a striking difference between Figures 5.23 and 5.24 is the disagreement in excitation energy values for which the same average neutron multiplicity is observed. For example, while in the $S+A u$ reaction an average multiplicity of 20 neutrons is observed at an energy of $600 \mathrm{MeV}$, in the $60 \mathrm{MeV} \mathrm{Au}$-induced reactions only 5 neutrons are observed for the same excitation energy. The reasons for this difference 
are not clear; because of the problems associated with the calibrations the results presented in this section should be considered only from a qualitative perspective and may not be regarded as quantitatively correct. Furthermore it is encouraging to point out that the results presented in sections 5.3.2, 5.3.3, and 5.3.4 are qualitatively consistent with the results from other experimental studies [Jia 88, Mor 88, Pi 91 ]. 


\section{Chapter 6}

\section{Discussion and Calculations}

In this chapter, possible mechanisms for multifragmentation are investigated by comparing some theories with the experimental data. In section 6.1 , a descripticn is given of the hybrid model (dynamical+statistical) used to simulate the $60 \mathrm{MeV} / \mathrm{A} \mathrm{Au}$ induced reactions. Results obtained with this model are shown and are compared with both inclusive and exclusive experimental data. Subsequently, in section 6.2 , the possibility of a phase transition in hot nuclei is investigated by examining the event-byevent moments of the fragment charge distributions. Finally, in section 6.3, a characteristic energy dependence for the multifragment decay probabilities of the source is presented similar to that observed for fission probabilities at low energies. Such a dependence could indicate that the multifragmentation process is statistical in nature.

6.1 A hybrid model approach: (dynamical + statistical) 
As was discussed in Chapter 2, dynamical models utilizing Boltzmann-NordheimVlasov, Boltzmann-Uehling-Uhlenbeck or Landau-Vlasov equations have been widely used to simulate the evolution of heavy-ion collisions at intermediate energies. These models however have not been able so far to reproduce the distribution of fragments in the mass range between the projectile and target. On the other hand, statistical theories, which successfully describe complex fragment emission in low-energy reactions, take minimal account of entrance channel effects and cannot reproduce the non-equilibrium features of intermediate-energy collisions. In this section we attempt to describe the complex-fragment production mechanism at intermediate energies with a hybrid model that incorporates both dynamical and statistical features [Co 92]. More specifically we will demonstrate that certain features of the experimental singles and coincidence events can be reproduced by terminating the dynamical calculation at a suitable time, after energy relaxation has occurred, and continuing the calculation with a compound nucleus decay code (GEMINI).

\subsubsection{Codes and Calculations}

The model calculations were performed in three steps. At each step a different computer code was used. A brief description of the codes utilized along with the calculations is presented below.

\section{Step 1: Dynamical calculations}

The early stages of the collision are simulated by solving the Landau-Vlasov(LV) equation, which includes the mean-field dynamics and the two-body interactions. The $\mathrm{LV}$ equation is given by: 


$$
\int \begin{gathered}
\frac{\delta f_{1}}{\delta t}+\frac{p_{1}}{m} \nabla_{r} f_{1}-\nabla_{r} U \nabla_{p} f_{1}= \\
d p_{2} d p_{3} d \Omega \frac{\left|p_{1}-p_{2}\right|}{m} \sigma_{N N}\left[f_{3} f_{4}\left(1-f_{1}\right)\left(1-f_{2}\right)\right. \\
\left.-f_{1} f_{2}\left(1-f_{3}\right)\left(1-f_{4}\right)\right] \delta\left(p_{1}+p_{2}-p_{3}-p_{4}\right)
\end{gathered}
$$

where $f$ is the one body Wigner function, $p$ is the momentum of a nucleon and $\sigma_{N N}$ is the nucleon-nucleon cross section, with an energy and angular dependence parametrized from experimental data [Sch 91]. The indexes 1,2,3, and 4 are used to label the quantities associated with two nucleons before and after they collide $: 1+2-->$ $3+4$. The mean field $U$ includes the Coulomb interaction between protons plus a nuclear potential approximated by a density-dependent, Skyrme-like interaction, which is given by the following equation :

$$
U_{\text {nuc }}(\rho)=A\left(\frac{\rho}{\rho_{0}}\right)+B\left(\frac{\rho}{\rho_{0}}\right) \sigma+C\left(\frac{\rho_{n}-\rho_{g}}{\rho_{0}}\right) \tau_{z}
$$

Here $\rho, \rho_{n}$ and $\rho_{p}$ are the local nucleon, neutron and proton densities, respectively; $\tau_{\mathbf{z}}$ is the isospin operator with the eigenvalues +1 or -1 for neutrons or protons respectively [Tsa 85]. The parameters A, B, C and $\sigma$ are chosen such as to reproduce nuclear matter saturation properties, and a compressibility coefficient of $\mathrm{K}=200 \mathrm{MeV}$ [Bon 90].

Equation (6.1) is solved by the test particle method [Ber 84, Ber 88]. In this method the one-body Wigner function of a system composed of $A_{p}$ nucleons for the projectile and $A_{t}$ nucleons for the target is described as an ensemble of $N_{G}\left(A_{p}+A_{b}\right)$ test particles that hit each other with a cross section $\sigma_{N N} / N_{G}$. To ensure a reasonable mapping of the phase space occupation and to avoid problems arising from numerical fluctuations each nucleon is represented by a large number $\left(N_{G}=40\right)$ of test particles. The Wigner function $f$ is approximated by 


$$
f(r, p, t)=(2 \pi \hbar)^{2} \sum_{i=1}^{N_{G}\left(A_{p}+A_{t}\right)} \delta\left(r-r_{i}\right) \delta\left(p-p_{i}\right)
$$

where $\delta$ is the Dirac delta function. In the test particle approach, $f$ solves the BNV equation provided the position $r_{i}$ and momentum $p_{i}$ are the solutions of equations of motion for the test particles in the mean field. The test particles propagate according to Hamiltonian dynamics under the influence of an acceleration term generated by the gradient of the mean field potential obtained in a self consistent way.

The test particles, which are generally described by the Dirac delta functions, are initially assigned random positions in a sharp sphere of nuclear radius $R$. Momentum is also randomly assigned to these test particles within a local sphere in momentum space of radius $p_{F}$. The radius $p_{F}$ is given by $p_{F}=\left(3 \pi^{2} \rho\right)^{1 / 3} \hbar$ where $\rho$ is the local density. Finally the momenta of the test particles in the projectile and target are boosted towards each other with their respective c.m. momenta determined from the incident energy and the masses of the projectile and target.

The dynamical code described above has been applied to the $60 \mathrm{MeV} / \mathrm{A} \mathrm{Au}+\mathrm{C}, \mathrm{Al}$, $\mathrm{V}$, and $\mathrm{Cu}$ reactions. A representative calculation is shown in Figure 6.1 for the $\mathrm{Au}+$ $\mathrm{Cu}$ system. The evolution of the density distribution as a function of time can be seen in both, the $\mathrm{X}-\mathrm{Y}$ and $\mathrm{Y}-\mathrm{Z}$ reaction planes for different impact parameters $\mathrm{b}$. The time increments are in steps of $20 \mathrm{fm} / \mathrm{c}$.

For the carbon and aluminum targets, the BNV calculations predict that in central collisions the two incident nuclei form a single composite system. The formation of a single hot nucleus occurs for impact parameters as large as $b=5 \mathrm{fm}$ in the aluminum case and $b=6 \mathrm{fm}$ in the carbon case and is reminiscent of complete fusion observed at low energies. At larger impact parameters $(b=5-7 \mathrm{fm})$, the simulations predict the 
formation of target and projectile-like fragments similar to an incomplete fusion process.

The LV calculations predict a substantial preequilibrium emission of nucleons. As an example a scatter plot of the time evolution of the $\mathrm{Au}+\mathrm{Al}$ collision for $\mathrm{b}=0$ as predicted by the LV calculations, shown in Figure 6.2, illustrates the important role that preequilibrium emission plays in the early stages of the reaction. Experimental investigations of heavy ion reactions at intermediate energies also show that dissipative collisions, ranging from complete fusion/incomplete fusion to deep inelastic processes, are preceded by a substantial amount of preequilibrium particle emission [Jou 91, Bor 88, Riv 88]. As will be discussed in Step 2, the preequilibrium emission process carries away a substantial amount of excitation energy.

For the heavier targets ( $\mathrm{V}$ and $\mathrm{Cu}$ ) the calculations predict that collisions at small impact parameters $b=0-4 \mathrm{fm}$ lead to the break up of the hot composite system. Several fragments (see Figure 6.1) are predicted to occur at impact parameter $b=0$ for both the vanadium and copper along with substantial preequilibrium emission. The onset of multifragmentation in these calculations could be due to regions of instabilities that the system encounters in its dynamical evolution. Such instabilities were discussed in Chapter 2.

At larger impact parameters $(b=5-7 \mathrm{fm})$, the simulations predict the formation of target and projectile-like fragments similar to an incomplete fusion process. A third fragment is also created in the overlap zone between the projectile and target. Finally, for impact parameters around 9-10 fm a dinuclear system is formed in a way reminiscent of deep inelastic collisions at low incident energy.

Preequilibrium emission of light particles accompanies these types of reaction (at $b=5-10$ ) as well. However the number of preequilibrium particles emitted decreases as a function of increasing impact parameter. 


\section{Step 2: The primary fragment observables.}

The dynamical model described above is a deterministic model, which does not account for all possible statistical decay channels and thus cannot correctly reproduce the late stages of the reaction where statistical decay is important. Therefore a coupling at some time teq is necessary between the dynamical stage and a subsequent statistical de-excitation stage. This time teq that we call "equilibration time" has to be long enough to assure that all the pre-equilibrium processes are already finished. In order to determine the equilibration time, we examine the emitted nucleon mean velocity as a function of time. As an example, Fig. 6.3 shows the time dependence of the emitted nucleon mean velocity in the center of mass at impact parameter $b=3$, for the 60 $\mathrm{MeV} / \mathrm{A} \mathrm{Au}+\mathrm{C}, \mathrm{Al}, \mathrm{V}, \mathrm{Cu}$ reactions. Clearly, for all targets, the mean velocity decreases as a function of time up to some equilibration time teq and remains constant afterwards, indicating that the subsequent emission (after $t_{e q}$ ) is not due to preequilibrium processes but instead arises from evaporation from an equilibrated source.

For the carbon target, the equilibration time occurs around $120 \mathrm{fm} / \mathrm{c}$ while for the heavier targets it occurs around $130 \mathrm{fm} / \mathrm{c}$. A similar value has been reported also by Jouan et al. [Jou 91], who found an equilibration time of $t \sim 120 \mathrm{fm} / \mathrm{c}$ for central collisions. It is important to note here that by the teq time, a great portion of the excitation energy has been carried away by the emitted preequilibrium nucleons. For example, in the carbon case, the excitation energy lost due to preequilibrium emission is almost $350 \mathrm{MeV}$ by $t_{e q}=120 \mathrm{fm} / \mathrm{c}$ (and $b=0$ ); this should be compared with the total available energy in the center of mass at $t=0 \mathrm{fm} / \mathrm{c}$ which is about $700 \mathrm{MeV}$. Furthermore, the excitation energy per nucleon of the composite system decreases from $E / A=3.6 \mathrm{MeV}$ at $\mathrm{t}=0 \mathrm{fm} / \mathrm{c}$ to $E / A=1.6 \mathrm{MeV}$ at $\mathrm{t}=120 \mathrm{fm} / \mathrm{c}$. For the copper target the 
total available excitation energy is about $2850 \mathrm{MeV}$ at $b=0 \mathrm{fm}$ and $t=0 \mathrm{fm} / \mathrm{c}$. At $t=130$ $\mathrm{fm} / \mathrm{c}$ however, the excitation energy has been reduced by approximately $50 \%$ due to a strong preequilibrium emission. A significant decrease in $\mathrm{E} / \mathrm{A}$ as a function of time is observed for this target as well.

Once the equilibration time has been determined, the next step is to define the primary fragments. Since it is desirable to define the primary fragments in a consistent way, a clustering procedure[Bo 90a] is used to calculate, for each impact parameter, the primary fragment observables: charge, excitation energy, mass, velocity, angle, and angular momentum. In this procedure two test particles $\mathrm{i}$ and $\mathrm{j}$ belong to the same cluster if they are sufficiently connected in $\mathbf{r}$ space, that is :

$$
\left|r_{i}-r_{j}\right|<D
$$

where $D$ is set to the minimum value that reproduces the mass of the target and projectile at $t=0(D=1.5 \mathrm{fm})$. The intrinsic angular momentum is calculated from $I=\Sigma$ $\mathrm{r}_{\mathbf{i}} \times \mathrm{p}_{\mathrm{i}}$ in the cluster center of mass. The excitation energy is computed as

$$
E^{*}=E_{\text {kin }}+E_{n m f}+E_{\text {coul }}-E_{g s}
$$

where $E_{k i n}$ is the total kinetic energy, $E_{n m f}$ is the nuclear mean field energy, $E_{\text {coul }}$ is the Coulomb energy and $E_{g s}$ is the ground state total energy calculated from the static solution used as initial condition for the BNV equation. As a representative example, Table 6.1 lists the values for the observables (mass, charge,...) of the primary fragments, determined with the above procedure in the $\mathrm{Au}+\mathrm{Cu}$ reactions, at different impact parameters. 


\section{Step 3: Statistical calculations.}

The final step in the calculations was to use the values of the observables (mass, charge,...) of the primary fragments as inputs to the statistical code GEMINI.

GEMINI is a statistical decay code that considers all decay channels from lightparticle emission to symmetric fission. It follows the de-excitation chains of individual nuclei through sequential binary decays until the resulting products are unable to undergo further decay. Details of the code are given in Appendix 2.

\begin{tabular}{llrrrl}
\hline $\mathrm{b}(\mathrm{fm})$ & $\mathrm{A}$ & $\mathrm{Z}$ & $\mathrm{E}^{*}(\mathrm{MeV})$ & $\mathrm{I}(\mathrm{h})$ & $\mathrm{V}_{\mathrm{s}}(1 / \mathrm{c})$ \\
\hline \multirow{3}{*}{0} & & & & & \\
& 62 & 27 & 151.0 & 5 & 0.27 \\
& 50 & 22 & 93.5 & 7 & 0.27 \\
& 44 & 20 & 84.8 & 5 & 0.27 \\
& 17 & 7 & 21.3 & 2 & 0.27 \\
& 14 & 6 & 2.7 & 2 & 0.27 \\
& 55 & 24 & 132.5 & 7 & 0.29 \\
& 46 & 20 & 126.0 & 4 & 0.26 \\
& 38 & 17 & 20.1 & 3 & 0.27 \\
& 31 & 13 & 118.5 & 5 & 0.27 \\
& 21 & 9 & 57.1 & 6 & 0.27 \\
& 87 & 38 & 189.0 & 13 & 0.29 \\
& 35 & 16 & 83.3 & 6 & 0.24 \\
& 28 & 12 & 120.5 & 8 & 0.25 \\
& 20 & 8 & 68.8 & 6 & 0.24 \\
& 17 & 8 & 5.0 & 1 & 0.27 \\
3 & 125 & 54 & 363.1 & 25 & 0.30 \\
& 29 & 12 & 79.0 & 6 & 0.25 \\
& 24 & 10 & 78.8 & 1 & 0.23 \\
& 22 & 10 & 13.0 & 3 & 0.27 \\
4 & 144 & 62 & 405.1 & 44 & 0.30
\end{tabular}




$\begin{array}{llllcl} & 36 & 16 & 189.6 & 11 & 0.22 \\ & 26 & 11 & 124.0 & 5 & 0.22 \\ 5 & 179 & 77 & 505.6 & 107 & 0.31 \\ & 28 & 12 & 150.7 & 11 & 0.15 \\ 6 & 174 & 75 & 404.5 & 90 & 0.31 \\ 7 & 178 & 76 & 357.5 & 99 & 0.32 \\ 8 & 176 & 75 & 158.0 & 73 & 0.34 \\ 9 & 179 & 75 & 77.0 & 68 & 0.35\end{array}$

Table 6.1. Mass, charge, excitation energy, angular momentum and velocity of the complex fragments at the equilibration time, for different impact parameters, for the reaction $\mathrm{Au}+\mathrm{Cu}$ at $60 \mathrm{MeV} / \mathrm{A}$.

\subsection{2) Comparisons with experimental data}

\section{a) Comparison with inclusive data.}

The inclusive complex fragment cross sections for the $60 \mathrm{MeV} / \mathrm{A} \mathrm{Au}+\mathrm{C}, \mathrm{Al}, \mathrm{V}$ and $\mathrm{Cu}$ reactions, calculated with the $\mathrm{LV}+\mathrm{GEMINI}$ codes, are shown in Figure 6.4. In the case of the carbon target, the inverted U-shape distribution is reproduced by the calculations. The predicted cross sections increase as a function of atomic number, peak around $\mathrm{Z}=40$, and then decrease; a similar patterm is observed in the experimental measurements. In addition, the calculations predict quite well the cross section values for fragments around $Z=40$ (symmetric fission events), while for fragments produced in binary decays far from symmetry the experimental and calculated points can differ by as much as a factor of 3 . 
For the Al target, the LV+GEMINI calculations predict the experimentally determined cross sections within an order of magnitude. However strong disagreement in the shape of the charge distribution is observed over almost the entire range of atomic numbers. For instance, while the calculations predict a drop in the cross section for the lower $Z s(10<Z<20)$ the experimental distribution increases for the lower $Z$ values. A possible explanation for this discrepancy is the fact that the LV calculations do not predict multifragmentation at small impact parameters for this target; instead complete fusion is predicted for the most central impact parameters. On the other hand the experimental data (coincidence $n$-fold events, $n=2,3,4,5$ ) demonstrates that the $\mathrm{Au}+$ $\mathrm{Al}$ reactions can lead to the production of several fragments (multifragmentation) in the exit channel.

For the heavier targets, the calculations (see Figure 6.4 ) reproduce reasonably well the shape of the charge distributions. Furthermore the calculations reproduce within a factor of 2 the magnitude of the experimental cross-sections over a large range of atomic numbers. The reasonable agreement between the calculated and experimental charge distributions can be attributed to the fact that the LV calculations do predict multifragmentation for the $60 \mathrm{MeV} / \mathrm{A} \mathrm{Au}+\mathrm{V}$, and $\mathrm{Cu}$ reactions.

Finally, the predicted cross sections do not depend strongly on the choice of the relaxation time. For instance, an increase of $20 \mathrm{fm} / \mathrm{c}$ in the relaxation time lowers the predicted cross sections at most by about $50 \%$. Thus, the overall agreement observed for all targets between the simulation and the data, indicates that this approach ( $\mathrm{LV}+$ GEMINI) describes correctly certain features of the mechanism responsible for the production of complex fragments.

\title{
b) Comparison with exclusive data
}

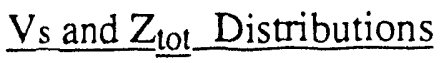


The (LV + GEMINI) model was utilized also to predict the source-velocity $\mathrm{V}_{\mathbf{s}}$ and total-charge $Z_{\text {tot }}$ distributions of the 2,3,4, and 5-fold events. The predictions were then compared with the experimental data. In this section, however, comparisons will be presented only for the $\mathrm{Cu}$ target for the following reasons : 1) In the case of the carbon no multifragmentation occurs. Although the calculations reproduce quite well the $\mathrm{V}_{\mathrm{s}}$ and $Z_{\text {tot }}$ distributions of the 2 -fold events, these events arise from the binary fission of the Au target, which is a well understood mechanism and is of little interest. 2) For the aluminum case the model calculations did not predict multifragmentation and therefore it was not possible to compare distributions arising from the higher n-fold events. 3) Finally, the results from the vanadium target are qualitatively similar to those from the copper and thus there is no need to report them here.

In order to account for the detector efficiency, the simulated events were filtered through a software replica of the detector. The simulated replica of the detection system accounted not only for fragments that hit the dead areas between the telescopes but also for high-velocity fragments that punched through the $5 \mathrm{~mm}$ Si detectors. Furthermore each event was constructed by including only fragments with $Z>5$ (in both calculations and experiment). The experimental total-charge and source-velocity distributions for the $\mathrm{Au}+\mathrm{Cu}$ reactions along with the quantities obtained from the calculations after filtering them through a software replica of our detector, are shown in Fig. 6.5.

For the 2-fold events, the calculation reproduces the peak position of the Ztot distribution, while the tail at low Ztot values is underestimated. For the higher $\mathrm{n}$-fold events ( $n=3,4$, and 5 ) the calculation overpredicts the peak positions. The shift observed in the Ztot peak position may be due to an underestimate of the excitation energy deposited in the primary fragments. Furthermore the widths of the Ztot distributions are underestimated. However, the calculations do predict the overall trend observed in the 
experimental distributions; i.e. the widths of the calculated distributions decrease progressively for higher $\mathrm{n}$-fold events.

The source-velocity distributions for the 2, 3, 4, and 5-fold events are plotted in the left hand column of Figure 6.5. They were obtained with the relation $V_{s}=\Sigma m_{i} \mathbf{v}_{i} / \Sigma m_{i}$, where $m_{i}$ and $v_{i}$ are the masses and velocities of the fragments of the detected or filtered events. In the case of the 2 -fold events the predicted source velocity distribution is in excellent agreement with the experimental data. The calculations predict remarkably well not only the value of the peak position but also the overall shape of the distribution. On the other hand, for the higher $n$-fold events the values of the peak positions of the Vs distributions are substantially underpredicted. In addition the widths of the Vs distribitions are also underestimated.

\section{Charge correlations}

In reference [Kre 92] multifragment emission in the $600 \mathrm{MeV} / \mathrm{A} \mathrm{Au}+\mathrm{Al}, \mathrm{V}$, and $\mathrm{Cu}$ reactions was studied by examining a set of observables as a function of the total charge $Z_{\text {total }}$. Calculations from several models were compared to the experimental data by utilizing this set of observables that included: the average charge of the largest fragment $\langle Z \max \rangle$, the average IMF (intermediate mass fragment) multiplicity $\left\langle\mathrm{M}_{\mathrm{IMF}}\right\rangle$, the average value of the relative asymmetry between the two largest fragments $\left\langle A_{2}>\right.$ and the average three body asymmetry $\left\langle A_{3}\right\rangle$. The results obtained by the models differed significantly from each other, establishing that such observables are sensitive to how the available phase space is populated and can be used to discriminate between models that have different treatments of nuclear disassembly. For instance, the sequential statistical model GEMINI predicted decays that were too asymmetric compared to the data, while the simultaneous statistical model predicted decays that were too symmetric. On the other hand a percolation model, which was 
adjusted to reproduce the mean multiplicity of the fragments and the size of $\mathrm{Zmax}$ predicted correctly the remaining dependences.

Motivated by the work of Kreutz et. al. [Kre 92] we have utilized the same observables in order to compare our model calculations with the experimental data frorn the $60 \mathrm{MeV} \mathrm{Au}+\mathrm{Cu}$ reaction. All calculations (LV + GEMINI) presented in thi: section have been filtered through a software replica of the detector.

\section{Average charge of the largest fragment $\left\langle Z_{\max }\right\rangle$ versus $Z_{t o t}$}

In figure 6.6 the average charge $\left\langle\mathrm{Z}_{\max }\right\rangle$ of the largest fragment is plotted as a function of the total charge $Z_{\text {tot }}$. A strong dependence is observed between the two variables; the average charge $\left\langle Z_{\max }\right\rangle$ of the largest fragment increases linearly with increasing total charge $Z_{\text {tot. }}$. Excellent agreement is obtained between the experimental points and the calculation over the entire range of Ztot.

A verage multiplicity of intermediate mass fragments $\left\langle M_{\text {imf }}>\right.$ versus $Z_{\text {tot }}$

In Figure 6.7 the average multiplicity of intermediate mass fragments(imf, $5<Z<40$ ) $<\mathrm{M}_{\mathrm{imf}}>$ obtained in the $60 \mathrm{MeV} / \mathrm{A} \mathrm{Au}+\mathrm{Cu}$ reactions is plotted as a function of the total charge. The average multiplicity $\left\langle\mathrm{M}_{\mathrm{imf}}\right\rangle$ increases smoothly with increasing Ztot, peaks at about Ztot=55 and then drops off. A similar behavior is predicted by the model calculations. However the calculated values are slightly higher(by about 10\%) than the values determined from the experiment.

Average relative asymmetry between the two largest fragments $\left\langle A_{2}\right\rangle$ versus $Z_{\text {tot }}$

In Figure 6.8 the average relative asymmetry $\left\langle A_{2}\right\rangle$ between the two largest fragments $Z_{1}$ and $Z_{2}\left(Z_{1}>Z_{2}\right)$ determined from the experimental coincidence data is plotted as a function of $Z_{\text {tot. }} A_{2}$ is defined as $A_{2}=\frac{Z_{1}-Z_{2}}{Z_{1}+Z_{2}}$ and is close to 0 for symmetric fragments while it reaches its upper limit of 1 for very asymmetric 
fragments. The average relative asymmetry increases smoothly as a function of the

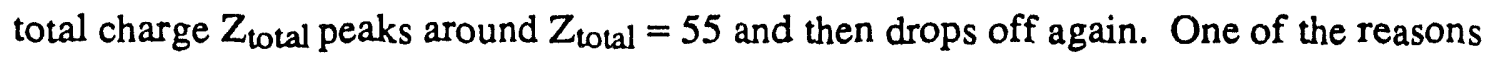
we do not observe large values of $A_{2}$ may be the absence of fragments with $Z<5$ from both the experimental data and the calculations. The BNV+ GEMINI model prediction is in good agreement the data especially at low Ztot values. Furthermore the model calculation reproduces remarkably well the overall trend of the data points. The predizted average relative asymmetry $\left\langle A_{2}\right\rangle$ increases smoothly at low $Z$ tot values, peaks around 55 and then drops off again.

Average three body asymmetry $\left\langle A_{3}\right\rangle$ versus $Z_{\text {tot }}$

Finally the model predictions and the data were compared by examining the three body asymmetry $\mathrm{A}_{3}$ which is defined as

$$
A_{3}=\frac{\left(\left(Z_{1}-\langle Z\rangle\right)^{2}+\left(Z_{2}-\langle Z\rangle\right)^{2}+\left(Z_{3}-\langle Z\rangle\right)^{2}\right)^{1 / 2}}{6^{1 / 2}<Z>} \text { where }\langle Z\rangle=\frac{Z_{1}+Z_{2}+Z_{3}}{3} \text {. The }
$$

quantity $\mathrm{A}_{3}$ has a maximum value near one for events characterized by a heavy residue along with two small fragments and has a value of zero when the three fragments are of equal size.

The average three body asymmetry $\left\langle\mathrm{A}_{3}\right\rangle$ determined from the experimental coincidence data is plotted in Figure 6.9 and shows a smooth increase as a function of $Z_{\text {iot }}$. One of the reasons we do not observe large values of $A_{3}$ may be due to the absence of fragments with $Z<5$. The calculations predict quite well the overall trend of the data and are in excellent agreement with the experiment at low $Z_{\text {tot }}$ values. However a small deviation for values of $Z_{t o t}$ larger than 40 is observed.

In summary we have attempted to reproduce the non-equilibrium and equilibrium features of complex fragment emission in intermediate energy heavy ion reactions by coupling a kinetic description of the dynamical stage of the collision with a subsequent 
statistical decay of the primary sources. The overall agreement obtained between the simulation and the experimental data indicates that such a dynamical-statistical coupling may be a useful tool for the understanding of the complex fragment production mechanisms.

An alternative way however, of studying the mechanisms responsible for the emission of multifragments would be to examine the data themselves in order to see whether they contain signatures that may be brought forth without the help or impediment, of any given model. In the following section possible signatures of a phase transition are presented by utilizing the moments of the charge distribution.

\subsection{Phase Transition?}

Early attempts to identify the origin of multifragmentation have relied mainly on information arising from the experimentally determined inclusive fragment

distributions. For instance the observation [Fin 82], made nearly one decade ago, that the fragment distribution measured in high-energy proton-induced reactions followed a power law $\left(\sim A^{-\tau}\right)$ was taken as an indication of the existence of a liquid-gas phase transition in finite nuclei. Since then, a similar dependence has been observed in a large number of studies and it has become common [Tra 92 and references therein] to fit a power-law function $A^{-\tau}$ to the inclusive light charge or $\operatorname{mass}(2<Z<12)$ yield distributions in order to obtain the critical exponent $\tau$. Figure 6.10 , is a compilation (taken from [Tra 92]) of power law parameters $\tau$, for a great variety of target-projectile combinations, extracted by fitting inclusive cross sections.

The predicted power law dependence $\left(\sim \mathrm{A}^{-\tau}\right)$ applies to fragment distributions arising only from events at the critical point. In the above studies, the parameter $\tau$ was 
extracted by fitting inclusive charge distributions that include fragments originating from events at the critical point as well as away from the critical point. This calls into question the applicability of the procedure utilized in the above studies to extract $\tau$. In order to avoid this difficulty we followed a different approach [Ca 88]; the critical exponent $\tau$ was extracted from the coincidence data by utilizing the moments of the experimental charge distribution. Before presenting the results, it may be useful to give a brief discussion of how the critical exponent $\tau$ is related to the moments of the charge distribution.

\subsubsection{Moment Analysis}

As was described in chapter 2, certain theories, such as percolation and chemical equilibrium, predict for infinite systems near the the critical point the production of clusters whose size distribution $N$ is given [Sta $71, \mathrm{Sta} 85, \mathrm{Her} 82$ ] by the general form:

$$
N(s, \varepsilon) \sim s^{-\tau} f\left(\varepsilon s^{\sigma}\right)
$$

where $s$ is the size of the clusters, $\tau$ and $\sigma$ are two critical exponents and $\varepsilon$ is a variable that characterizes the state of the system. In thermal phase transitions $\varepsilon=T-T_{c}$ where $T c$ is the critical temperature, while in percolation theory $\varepsilon=p-p_{c}$ and $p_{c}$ is the critical fraction of active bonds or occupied states. $f\left(\varepsilon s^{\sigma}\right)$ is a scaling function that decays exponentially for large values of $|\varepsilon|$ and is equal to 1 when $\varepsilon=0$; therefore when the system is at criticality $(\varepsilon=0)$ equation 6.6 reduces to a power law.

The $k$-th moment of the fragment size distribution $N(s, \varepsilon)$ is given [Sta 85 ] by the following equation 


$$
M_{k}=\sum_{s=1}^{\infty} s^{k} N(s, \varepsilon)
$$

where $s$ is the size of the fragments and the summation runs over all finite size fragments. By utilizing equation (6.6), equation (6.7) becomes

$$
\begin{aligned}
M_{k} & \propto \sum_{j} s^{k} s^{-\tau} f\left(\varepsilon s^{\sigma}\right) \\
& \propto \int s^{k} s^{-\tau} f\left(\varepsilon s^{\sigma}\right) d s \\
& \propto \varepsilon^{(\tau-1-k) / \sigma} \int z^{k-\tau} f(z) d z \\
& \propto \varepsilon^{(\tau-1-k) / \sigma},
\end{aligned}
$$

where we have replaced the summation with an integration over $s$ and have used $z=\varepsilon s^{\sigma}$. (The integral over $z$ runs from 0 to $\infty$ and therefore $\int z^{k-\tau} f(z) d z$ is a constant. For instance in the case of the Fisher droplet model[Fis 67] $f(z)$ is assumed to be $f(z) \propto s^{-\tau}$ $\exp (-$ const $\cdot z)$ and the integral is then just proportional to the gamma function $\Gamma(\mathrm{k}-$ $\tau+1)$ ) Equation 6.8 relates the values of the critical exponents $\sigma$ and $\tau$ to the moments of the charge distribution. Therefore it may be possible to extract the critical exponent $\tau$ from the moments $\mathrm{M}_{\mathrm{k}}$ of the experimental charge distributions.

The experimental charge distribution however, is associated with events that are characterized by a range of $\varepsilon$ values. On the other hand the moments in equation 6.7 were defined by using a distribution $\mathrm{N}(\mathrm{s}, \varepsilon)$ which is characterized by one (constant) value of $\varepsilon$. Therefore equation 6.7 cannot be applied directly to the data since $\varepsilon$ is not a measurable quantity. In order to avoid this difficulty, the moments of the fragmentsize distribution were determined on an event by event basis as proposed by Campi [Cam 86, Cam 88]. Every event $i$ can be associated to a quantity $M_{k}^{i}$ which is defined 


$$
\mathbf{M}_{\mathrm{k}}^{\mathrm{i}}=\sum_{\mathrm{s}} \mathrm{s}^{\mathrm{k}} \mathrm{m}^{\mathrm{i}}(\mathrm{s})
$$

where $\mathrm{m}^{\mathrm{i}}(\mathrm{s})=7,1,2 \ldots$ is the number of fragments of size $\mathrm{s}$ that appear in the event $i$. Here th: sum runs over all fragments, except the heaviest one produced in the event. The largest fragment is excluded in analogy with the infinite percolating cluster in the percolation case and the condensate in the case of a liquid-gas phase transition (see Chapter 2). In the limit of a large number of events, all of which are characterized by the same $\varepsilon$, the average value of $M_{k}^{j}$ should approach the actual value of $M_{k}$ of the distribution $N(s, \varepsilon)$.

The advantage of utilizing equation 6.10 is that $\mathrm{M}_{\mathrm{k}}^{\mathrm{i}}$ can be computed for an event $\mathrm{i}$ without the knowledge of $\varepsilon$ for this event.

\section{$\ln \mathrm{M}_{3}$ vs $\ln \mathrm{M}_{2}$}

The second and third moments were determined by means of eqn. 6.10 for each of the $n$-fold $(n=3,4,5)$ events produced in the $60 \mathrm{MeV} / \mathrm{A} \mathrm{Au}+\mathrm{Cu}$ reactions. In order to reduce the contamination arising from incompletely detected events only events with Ztot $>35$ were considered.

From equation 6.8 we see that the points $\left(\mathrm{M}_{3}, \mathrm{M}_{2}^{\mathrm{i}}\right)$ should fall on a straight line in a plot of $\ln M_{2}$ vs. $\ln M_{3}$. The value of the critical exponent $\tau$ can then be determined from the slope $\mu$ of the line, which is given by

$$
\begin{aligned}
\mu & =\frac{d\left(\ln M_{3}\right)}{d\left(\ln M_{2}\right)} \\
& =\frac{((\tau-1-3) / \sigma d(\ln \varepsilon))}{((\tau-1-2) / \sigma d(\ln \varepsilon))} \\
& =\frac{\tau-4}{\tau-3} .
\end{aligned}
$$


Figure 6.11 shows the dependence between $\ln M_{2}^{i}$ and $\ln M_{3}^{i}$; a strong linear correlation is observed between the two quantities. The linear distribution of the events in the $\ln M_{2}^{i}$ vs $\ln M_{3}^{i}$ plane can be associated with an average line whose slope has a value $\mu=1.76 \pm 0.15$. By inserting this value in equation (6.9) the critical exponent $\tau$ is found to be equal to $1.7 \pm 0.1$. This value is close to the $\tau$-parameter values that have been extracted by fitting the inclusive light fragment distributions with a power law function (see Figure 6.11). For comparison we mention that the value $\tau$ in a liquid-gastype phase transition is predicted to be $\tau=2.5$, while in percolation theory it is found to be $\tau=2.2[$ Cam 86].

However, further investigation has shown that the strong linear correlation observed in Figure 6.11 is not only characteristic of a power law distribution, but can arise from other distributions as well. For instance Figure 6.12 shows the coincidence charge distribution from which the moments $M_{2}$ and $M_{3}$ were determined. The coincidence charge distribution is not characterized by a power law form, although a best fit through the points with a power law function gives $\tau=1.7$. This limitation calls into question the approach of extracting information from a plot of $\ln \mathrm{M}_{2}$ vs $\ln \mathrm{M}_{3}$.

\section{$\ln \left(Z_{\max }\right)$ vs $\ln \left(M_{2} / M_{1}\right)$}

The critical behavior of nuclei can be investigated further by examining the correlation between the heaviest fragment $Z_{\max }$ and $\ln \left(\mathrm{M}_{2} / \mathrm{M}_{1}\right)$. In this case one expects to see a two-branch feature [Cam 88, Jaq 91] which is believed to reflect the occurrence of a liquid-gas phase transition in nuclear matter. This feature is characterized by an upper branch with a negative slope and a lower branch with a positive slope. The upper branch is associated with subcritical events; that is events with temperature $T$ less than the critical temperature $T$. On the other hand the lower branch corresponds to events 
with temperature $\mathrm{T}$ greater than the critical temperature $\mathrm{Tc}$; these events are called supercritical events. The two branches are expected to meet at the critical point.

In the upper- leftmost portion of Figure 6.13 a contour plot of $\ln \left(Z_{\max }\right)$ versus $\ln \left(\mathrm{M}_{2} / \mathrm{M}_{1}\right)$ is shown for $\mathrm{Au}+\mathrm{Cu}$ at $60 \mathrm{MeV} / \mathrm{A}$. The distribution of the events populates a large portion of the available phase space and the correlation is very broad. Similar correlations have been observed in References [Kre 92, Jak 90]. The lack of a strong correlation is due to the large fluctuations of $\mathrm{Zmax}$ in the finite system $(\mathrm{Au}+\mathrm{Cu})$. Nevertheless, by utilizing the average of $\ln \left(\mathrm{M}_{2} / \mathrm{M}_{1}\right)$ two correlated branches can be roughly obtained. Figure 6.14 shows $\ln \left(\mathrm{Z}_{\max }\right)$ as a function of $\left\langle\ln \left(\mathrm{M}_{2} / \mathrm{M}_{1}\right)>\right.$ were the two branches are clearly visible. In determining the averages $\left\langle\ln \left(\mathrm{M}_{2} / \mathrm{M}_{1}\right)\right\rangle$, events arising from symmetric fission were not included; these events are well separated from the two branches and are marked by large values of $\ln \left(\mathrm{Z}_{\max }\right)$ and $\ln \left(\mathrm{M}_{2} / \mathrm{M}_{1}\right)$. The need to remove such events before calculating these averages has been emphasized by Jaqaman and Gross[ Jaq 91]. Jaqaman et. al. suggest that in order to compare the phase transition in the nuclear case with the liquid-gas or percolation phase transition, it is important that the fission events are excluded from the analysis. However such an approach is not justified since the 2,3,4 and 5-fold events may arise from similar mechanisms. Therefore it would be more fitting to include the fission events in the analysis.

Figure 6.13 shows the evolution of all the events in the $\ln \left(Z_{\max }\right)-\ln \left(\mathrm{M}_{2} / \mathrm{M}_{1}\right)$ plane, including the fission events, as a function of excitation energy. The numbers in each of the subplots represent the average excitation energy per nucleon of the events. The excitation energy was calculated from the source velocity by means of equation 5.6 (see section 5.2.5). At very low energies ( $1 \mathrm{MeV} / \mathrm{A}$ ) only the upper branch associated with the subcritical events is populated. The high intensity of contour lines around the point $(3.5,3.7)$ can be associated with events arising from the fission of the Au-like 
projectiles produced in peripheral collisions. As the excitation energy per nucleon increases(5-7 MeV/A) the events fill up the whole available area and a smooth transition is observed between the two branches. At high excitation energies $(9 \mathrm{MeV} / \mathrm{A})$ only the lower branch associated with supercritical events is populated; these events are characterized by small values of $Z$ max. This evolution may indicate the presence of a phase transition in multifragmentation.

\section{$\left\langle\gamma_{2}>\right.$ vs $Z_{\text {tot }}$}

Information regarding the "phase transition" of nuclear matter can be obtained also by examining the following combination of moments[Cam 88]

$$
\gamma_{2}=\frac{M_{2} \times M_{0}}{M_{1}^{2}}
$$

which can be re-expressed in terms of the normalized charge variance

$$
\gamma_{2}=\frac{\sigma^{2}}{\langle Z\rangle^{2}}+1
$$

where $\sigma$ is the variance of the charge distribution within the event and $\langle\mathrm{Z}\rangle$ is the average charge of the event. When all the charges in the event are of the same size, then $\gamma_{2}$ reaches its lower limit of 1 . This limit is approached for three types of events: a) those with light evaporated fragments, b) symmetric fission of Au-like projectile, and c) total disassembly of the system. A large value of $\gamma_{2}$ means that the fragments of the event are quite different in size.

Although in percolation theory $\gamma_{2}$ diverges for infinite systems at the critical point, in finite systems it is predicted to show a smooth peak. In Figure 6.15 we have plotted $<\gamma_{2}>$ versus $Z_{\text {tot }}$ for the $60 \mathrm{MeV} / \mathrm{A} \mathrm{Au}+\mathrm{Cu}$ reactions. A peak is observed in the experimentally determined $\left\langle\gamma_{2}\right\rangle$ distribution; this may be an indication of a phase 
transition. A similar behavior has been observed in References [ Kr 92, Jak 90]. The height of the distribution is reduced primarily by the large number of two-fold events (since they have a value of $\gamma_{2}=1$ ) and by our experimental threshold $Z>5$. As for the calculated values (from LV + GEMINI) they show no strong indication of a peak.

In summary we have searched for possible signals of a phase transition by examining various combinations of the conditional moments of the charge distribution arising from the coincidence events. The overall event-by-event moment analysis suggests that the hot nuclear systems formed in the $60 \mathrm{MeV} \mathrm{Au}$-induced reactions may break up into 3, 4 and 5 fold events by undergoing a phase transition.

\subsection{Statistical Multifragmentation ?}

In reference [Mor 69] the statistical nature of the rise of the fission probability $P$ with excitation energy $E$ has been demonstrated [Mor 69] by the presence of a linear dependence between the variables $\ln (\mathrm{P})$ and $\mathrm{E}^{-1 / 2}$. In this section we apply a similar approach to intermediate-energy heavy-ion reactions in order to demonstrate the statistical nature of the multifragmentation branching ratios.

\subsection{1) The method}

As was discussed in Chapter 2, the binary decay of a nucleus is determined by a barrier associated with a saddle point in the nuclear potential energy surface of the nucleus. Let us assume that a similar barrier exists for higher-order decays and let $B_{2}$, $B_{3}, \ldots B_{n}$ be the average barriers associated with binary, ternary, and $n$-body decays. There might exist a class of barriers such that all the binary configurations would have 
barriers closer to each other than to those of the ternary configurations, and so on. Then, the decay probability for each exit channel is given by

$$
P_{n}(E) \propto \rho\left(E-B_{n}\right)
$$

where $\rho\left(E-B_{n}\right)$ is the level density of the system at an excitation energy equal to the available energy $E$ minus the barrier $B_{n}$. For a Fermi gas level density equation 6.14 becomes

$$
P_{n}(E) \propto e^{2 \sqrt{a\left(E-B_{n}\right)}}
$$

where $a$ is the level density parameter. When the available energy $\mathrm{E}$ is much greater than the barrier $B_{n}$ the Taylor expansion of $(E-B n)^{-1 / 2}$ in powers of $B n$ can be utilized, and one obtains from eqn 6.15 :

$$
P_{n}(E) \propto e^{2 \sqrt{a E}} e^{-B_{n} \sqrt{(a / E)}} \propto e^{-B_{n} / T}
$$

By constructing the ratio of the $n$-fold events to the binary events we obtain

$$
\ln \left(P_{n} / P_{2}\right) \propto-\sqrt{a / E}\left(B_{n}-B_{2}\right)
$$

This suggests that a plot of

$$
\ln \left(P_{n} / P_{2}\right) \text { vs. } E^{-1 / 2}
$$

should give a straight a line. 
In reference [Mor 69] this dependence was empirically confirmed for the overall fission probabilities in the $\mathrm{Pb}$ region and was then used to prove that the sharp rise of the fission cross section in $e^{-}$induced reactions of similar nuclei is also of a statistical nature. Figure 6.16 a shows the total fission probability as a function of $E^{-1 / 2}$ for three $\alpha$-induced reactions : ${ }^{206} \mathrm{~Pb}\left({ }^{4} \mathrm{He}, \mathrm{f}\right),{ }^{197} \mathrm{Au}\left({ }^{4} \mathrm{He}, \mathrm{f}\right)$ and ${ }^{184} \mathrm{~W}\left({ }^{4} \mathrm{He}, \mathrm{f}\right)$. For all reactions a linear dependence is observed. The slopes of the lines can be associated quantitatively with the known fission barriers. In figure $6.16 \mathrm{~b}$ a similar plot is shown for the photofission cross-sections of ${ }^{206} \mathrm{Bi},{ }^{208} \mathrm{~Pb},{ }^{174} \mathrm{Yb}$, and ${ }^{154} \mathrm{Sm}$ obtained by the unfolding of the respective electron-induced fission cross sections from the virtual photon spectra. The linear distribution of the points and the close relation of the slopes with the fission barriers, confirmed that the rise of the photofission cross section with increasing photon energy is a statistical effect determined from the phase space associated with the competing decay channels.

In this section we apply a similar approach to the $60 \mathrm{MeV} / \mathrm{A} \mathrm{Au}+\mathrm{Al}, \mathrm{V}$, and $\mathrm{Cu}$ reactions in order to determine if the nature of the multifragmentation branching ratios can be characterized as statistical.

\subsection{2) Results}

To determine whether a linear dependence exists in the data we have plotted in Figure 6.17 , for the $60 \mathrm{MeV} / \mathrm{A} \mathrm{Au}+\mathrm{Al}, \mathrm{V}$, and $\mathrm{Cu}$ reactions, the natural logarithm of the corrected probabilities $P(n) / P(2)(n=3,4,5)$ as a function of $E^{-1 / 2}$, where $E$ is the excitation energy of the decaying source. The method used to obtain the corrected probabilities is model dependent and was outlined in Chapter 5 (section 5.2.6). In Chapter 5 it was also emphasized that it was necessary to gate on the Ztot (Ztot $>35)$ of each event in order to reduce the contamination arising from incomplete events. 
However since the probabilities are corrected for detection efficiency, no such gate was required.

An interesting feature is that the data from all reactions fall on the same curves. This indicates, that once the multifragmentation source is characterized in terms of the kinematically determined excitation energy, the branching ratios for the various multifragment channels are independent of the specific reaction that has produced the source. This decoupling between the entrance and exit channel suggests that the dynamics of the reaction may be limited to the formation of a source of a given mass, energy and angular momentum through a mechanism similar to incomplete fusion. Once this source is formed, its decay is independent of its mode of formation.

Furthermore Figure 6.17 shows that the points for all three types of decay $(3,4$, and 5-body decays) fall on straight lines, suggesting that the branching ratios between the various multifragmentation channels may be controlled by the available phase space.

Finally, for completeness we mention that the above method does not discriminate between prompt and sequential statistical decay. For instance, if the system undergoes sequential decay with probabilities that are much smaller than 1 then the probability to obtain $\mathrm{n}$ fragments is given by:

$$
\begin{aligned}
P_{n}(E) & \propto K(n) e^{-b_{1} / T_{1}} e^{-b_{2} / T_{2} \ldots} \propto K(n) e^{-\left(b_{1}+b_{2} \ldots\right) / T} \propto K(n) e^{-B_{n} / T} \\
& \propto K(n) e^{-B_{n} \sqrt{(a / E)}}
\end{aligned}
$$

where $b_{1}, b_{2}, b_{3}, \ldots b_{n}$ are the barriers for the successive binary decays, $K(n)$ is a combinatorial factor and $B_{n}=b_{1}+b_{2}+\ldots \ldots$. From this last relation (6.19), we see that even for multiple sequential binary decay we expect a linear dependence of $\ln P_{n}$ versus $E^{-1 / 2}$. Therefore the observed linear dependence does not distinguish between 
simultaneous and sequential statistical decay. But since the barrier Bn can be obtained from the slope of the straight line, which is given by

$$
\frac{d}{d E-1 / 2}\left(\ln \frac{P_{n}}{P_{2}}\right)=-\sqrt{a}\left(B_{n}-B_{2}\right)
$$

and $B_{n}$ could be very different for simultaneous and sequential decay, further work with both the data and the models might lead to a differentiation between the two decay mechanisms. 


\section{Chapter 7}

\section{Conclusion}

The goal of this thesis was to study the emission of complex fragments in the 60 $\mathrm{MeV} / \mathrm{A} \mathrm{Au}+\mathrm{C}, \mathrm{Al}, \mathrm{V}, \mathrm{Cu}$ and $\mathrm{Au}$ reactions. In this section we summarize the results of this work and present our conclusions.

\section{$60 \mathrm{MeV} / \mathrm{A}{ }^{197} \mathrm{Au}+{ }^{12} \mathrm{C}$}

In the $60 \mathrm{MeV} / \mathrm{A} \mathrm{Au}+\mathrm{C}$ reactions, the complex fragments are produced mainly in highly equilibrated binary processes; in particular, they arise from the compound binary decay of Au-like nuclei. Although the complex fragments may be emitted from a range of sources formed in incomplete fusion processes, the small size of the carbon target limits the range of mass transfers that can occur from the target to the projectile. Therefore the sources that can be formed in the $\mathrm{Au}+\mathrm{C}$ reactions are very similar in size with the Au-like projectile.

The relaxed nature of the decay process has been determined from the angular distributions and emission velocities of the fragments. The fragment angular distributions are flat $(\mathrm{d} \sigma / \mathrm{d} \theta=\mathrm{const}$.) over a large range of atomic numbers, and 
demonstrate the complete relaxation of the angular degrees freedom. The fragment emission velocities are consistent with the Coulomb repulsion in a binary decay.

The binary nature of the decay process has been illustrated by the well defined Coulomb rings and by the 2 -fold coincidence events $(Z 1+Z 2)$ which sum up to values around the atomic number $(Z=79)$ of $\mathrm{Au}$. Furthermore the shape of the charge distribution is consistent with fission from a compound system above the BusinaroGallone point.

Higher $n$-fold events ( $n=3$, and 4 ) have also been observed but are less than $2 \%$ of the total coincidence events. This is because the small mass of the carbon limits the range of mass transfers that can occur from the target to the projectile and therefore also limits the formation of highly excited nuclear systems that would ultimately decay by multifragmentation.

\section{$60 \mathrm{MeV} / \mathrm{A}{ }^{197} \mathrm{Au}+{ }^{27} \mathrm{Al},{ }^{51 \mathrm{~V}},{ }^{63} \mathrm{Cu}$, and ${ }^{197} \mathrm{Au}$}

In the $60 \mathrm{MeV} / \mathrm{A}{ }^{197} \mathrm{Au}+{ }^{27} \mathrm{Al}, 51 \mathrm{~V}, 63 \mathrm{Cu}$, and ${ }^{197} \mathrm{Au}$ reactions, complex fragments are produced not only in binary processes, but also in multifragmentation. This is because the larger range of mass transfers available from the heavier targets gives rise to a larger range of excitation energies that favor multifragmentation relative to binary decay.

Although significant multifragmentation decay leads to a filling-in of the Coulomb rings, the flat isotropic angular distributions of the fragments along with their Coulomb like emission velocities suggest the presence of a strong compound binary component in the inclusive data. This component is associated with the compound nucleus decay of Au-like projectiles generated in peripheral collisions and dominates the singles crosssections. 
For these targets, the shape of the charge distributions cannot be associated completely with statistical emission from systems either above or below the Businaro Gallone point. Although most of the fragments produced in these reactions are attributed to the decay of Au-like sources produced in peripheral reactions, a significant yield of lighter fragments $(Z<25)$ is due to multibody decay. The yield of lighter fragments is comparable in magnitude to the fission fragment yields $(Z \sim 40)$, and increases as a function of the mass of the target; due to the large available center of mass energy, multifragmentation becomes increasingly important for the more symmetric entrance channels.

In order to untangle the various decay processes we have used the source velocity Vs as a measure of the excitation energy $E^{*}$. The excitation energy $E^{*}$ was calculated from the source velocity within the incomplete fusion picture. High source velocities and low excitation energies are associated with peripheral reactions and lead predominantly to the binary decay of Au-like projectiles. As we progressively move to lower source velocities, the characteristic binary signature disappears, and the highly excited nuclear systems disintegrates preferentially into several small fragments.

Excitation functions for the multifragment events were constructed over a large range of excitation energies and for all targets. The excitation functions increase substantially with excitation energy $E^{*}$. Furthermore the data from all reactions fall on the same curves. This indicates that the dynamics of the reaction may be limited to the formation of a source through a mechanism similar to incomplete fusion.

\section{Neutron Calorimeter}

The neutron multiplicity is utilized in the present experiment as an independent measure of the deposited excitation energy; low neutron multiplicities are associated with low excitation energies generated in peripheral reactions, while high neutron 
multiplicities arise from the more central collisions and are characterized by high excitation energies. Furthermore, massive fragments are unlikely to survive the most dissipitative, central collisions selected by high neutron multiplicity gates. Instead, for these events the nuclear system is observed to disassemble in small fragments and a huge number of light particles.

An interesting dependence between the average neutron multiplicity $\langle M n\rangle$ and the excitation energy $\mathrm{E}^{*}$ of the decaying system was also determined by examining directly the relationship between $\mathrm{E}^{*}$ and $\mathrm{Mn}$. For all targets the average neutron multiplicity increases initially as a function of energy, it reaches a saturation value and remains approximately constant with increasing excitation energy. This behavior can be attributed to charged particle emission that becomes increasingly important with increasing excitation energy. Therefore the neutron multiplicity may not be an accurate measure of $\mathrm{E}^{*}$ at high excitation energies because of the increasing competition of charged particle emission.

\section{Landau-Vlasov + Gemini Calculations}

In an attempt to reproduce the $60 \mathrm{MeV}$ Au-induced reactions we have coupled a kinetic description of the dynamical stage of the collision with a subsequent statistical decay of the primary sources. The dynamical evolution of the $\mathrm{Au}+\mathrm{C}, \mathrm{Al}, \mathrm{V}$, and $\mathrm{Cu}$ reactions at $60 \mathrm{MeV} / \mathrm{A}$ is simulated by solving the BNV equation up to a time teq. This time teq that we call "equilibration time" must be long enough to assure that all the preequilibrium processes are already finished. At teq a clustering procedure is used to determine, for each impact parameter, the primary fragment mass, charge, velocity, angle, exitation energy and angular momentum. The final step in the calculations is to use the values of the mass, charge,... of the primary fragments as inputs to the statistical code GEMINI. 
The calculations predict reasonably well certain features of the inclusive and exclusive data. For instance the shape of the inclusive charge distributions from the $\mathrm{Au}$ $+\mathrm{C}, \mathrm{V}$, and $\mathrm{Cu}$ reactions is well reproduced. Furthermore the calculations predict within a factor of 2 the magnitude of the experimental cross-sections over a large range of atomic numbers. However, for the $\mathrm{Al}$ target, strong disagreement in the shape of the charge distribution is observed over almost the entire range of atomic numbers.

Comparisons with the coincidence data, show that the $\mathrm{V}_{\mathrm{s}}$ and $\mathrm{Z}_{\text {total }}$ distributions of the two-fold events are well reproduced; however the calculations do not adequately predict the $V_{s}$ and $Z_{1 o t a l}$ distributions of the 3-, 4-, and 5-fold events. Furthermore, reasonable agreement between the calculations and the data is obtained for a set of observables. These observables have been successfully used in other studies to discriminate between models of nuclear disassembly and include: the average charge of the largest fragment $\langle Z \max \rangle$, the average IMF (intermediate mass fragment) multiplicity $\left\langle\mathrm{M}_{\mathbb{I} M F}\right\rangle$, the average value of the relative asymmetry between the two largest fragments $\left\langle A_{2}>\right.$ and the average three body asymmetry $\left\langle A_{3}>\right.$.

The overall agreement obtained between the simulation and the experimental data suggests that such a dynamical-statistical coupling may be a powerful tool for the understanding of multifragmentation.

\section{Phase Transition}

The possibility that the system undergoes a phase transition (percolation-like or liquid vapor-like) has been investigated by examining observables that behave qualitatively differently whether a phase transition is present or not. These observables were constructed from the event-by-event moments of the fragment charge distributions. 
This analysis shows that the correlation between $\ln \left(\mathrm{Z}_{\max }\right)$ and $\left\langle\ln \left(\mathrm{M}_{2} / \mathrm{M}_{1}\right)\right\rangle$ is characterized by two clear branches that can be used to classify events as sub- and super-critical events. The evolution of the experimental events in the $\ln \left(Z_{\max }\right)$ $\ln \left(\mathrm{M}_{2} / \mathrm{M}_{1}\right)$ plane as a function of excitation energy shows a changing population of sub- to super-critical events that is consistent with a percolation-like critical behavior. Furthermore a peak is observed in the experimentally determined $\langle\gamma\rangle$ distribution that may be taken as an indication of a phase transition.

The overall event by event moment analysis suggests that the hot nuclear systems formed in the $60 \mathrm{MeV}$ Au-induced reactions may break up into 3, 4 and 5 fold events by undergoing a phase transition.

\section{Statistical Multifragmentation}

The possibility that the break-up of the system into several fragments may be determined by some kind of a barrier was also examined. We searched for statistical effects by utilizing a generic attribute of statistical decay that has been verified with well understood fission reactions. In low-energy reactions the statistical nature of the rise of the fission probability $P$ with excitation energy $E$ has been determined by the presence of a characteristic energy dependence. A similar approach was applied to the 3-,4-,and 5-fold events from the $60 \mathrm{MeV} / \mathrm{A} \mathrm{Au}+\mathrm{Al}, \mathrm{V}$, and $\mathrm{Cu}$ reactions .

The points for all three types of decay(3-, 4-, and 5-body decays) fall on straight lines, demonstrating the statistical nature of the multifragment branching ratios. The qualitative features of the excitation functions do not permit distinguishing between a sequential or simultaneous decay mechanism, but the quantitative features may contain relevant information in this regard. 


\section{Appendix 1}

The fission decay width is calculatea according to the Bohr-Wheeler formalism which makes use of the transition state method. The fission decay width is given by:

$$
\Gamma_{f}=\frac{1}{2 \pi \rho(E)} \int_{0}^{E-B_{f}} \rho^{*}\left(E-B_{f}-\varepsilon\right) d \varepsilon,
$$

where $\rho(E)$ and $\rho^{*}\left(E-B_{f}-\varepsilon\right)$ are the level densities of the compound nucleus and of the fission saddle point; $\varepsilon$ is the kinetic energy along the fission mode and $B_{f}$ is the fission bartier. The transition-state model of complex fragment emission, as developed by Moretto, generalizes the Bohr-Wheeler formalism by extending the saddle point to a ridge line of $Z$-dependent conditional saddles by utilizing the charge-asymmetry coordinate $Z_{a s y}=Z / Z_{t o t}$, where $Z$ is the emitted fragment charge and $Z$ tot is the compound nucleus charge. In this case, the decay width at any conditional saddle is proportional to the number of states above the conditional barrier, and is given by:

$$
\Gamma_{z}=\frac{1}{2 \pi \rho(E)} \int_{0}^{E-B_{z}} \rho^{*}\left(E-B_{z}-\varepsilon\right) d \varepsilon
$$

where $\rho(E)$ is the compound nucleus level density and $\rho^{*}\left(E-B_{Z}-\varepsilon\right)$ is the level density at the conditional saddle of energy $\mathrm{Bz}$, which the system is transiting with kinetic energy $\varepsilon$. Equation ( $A 1.2$ ) can be simplified by expanding $\rho^{*}\left(E-B_{Z}-\varepsilon\right)$ about $\varepsilon=0$ : 


$$
\begin{aligned}
\ln \left(\rho^{*}\left(E-B_{Z}-\varepsilon\right)\right) & =\ln \left(\rho^{*}\left(E-B_{Z}\right)+\frac{d \ln \rho^{*}\left(E-B_{Z}\right)}{d E}(E-B Z-\varepsilon-E+B)+\ldots \ldots\right. \\
& =\ln \left(\rho^{*}\left(E-B_{Z}\right)+(-\varepsilon) / T+\ldots .\right.
\end{aligned}
$$

where $\frac{d \ln \rho^{*}\left(E-B_{Z}\right)}{d E}=1 / T$, and $T$ is the nuclear temperature at the saddle point. From eqn. (A.I.2) and (A.I.3) we get :

$$
\begin{aligned}
\Gamma_{z} & \approx \frac{1}{2 \pi \rho(E)} \rho^{*}(E-B z) \int \exp (-\varepsilon / T) d \varepsilon \\
& \approx \frac{T}{2 \pi \rho(E)} \rho^{*}(E-B z)
\end{aligned}
$$

Equation (A.I.4) can be further simplified, if in the limit of high excitation energy $\mathrm{E}$ we expand $\rho^{*}(E-B z)$ about $B z=0$ to obtain:

$$
\Gamma_{z} \approx \frac{T}{2 \pi \rho(E)} \rho^{*}(E) e^{-B z / T} \approx \frac{T}{2 \pi} e^{-B z / T} \propto e^{-B z / T}
$$

In the above equation $p(E)$ and $\rho *(E)$ cancel out because at high excitation energy $\rho(E)=\rho^{*}(E) . T$ is calculated at an excitation energy $E x=E-B z=a T^{2}$, where $a$ is the nuclear level density parameter, usually taken to be in the range of $A / 10$ to $A / 8$.

From (A.1.5) we see that the mass- or charge-yield mirrors the ridge line, being characterized by high emission probabilities in the regions of low potential energy and vice-versa. 


\section{Appendix 2}

Statistical model calculations were performed with the Monte Carlo computer code GEMINI[Cha 88]. This code follows the decay of a compound nucleus. All possible binary decays from light-particle emission to symmetric division are considered. After each binary division, further decay of the resulting excited fragments is followed until all of the available excitation energy is exhausted.

The decay width for the evaporation of fragments with $\mathrm{Z}<2$ is calculated using the Hauser-Feshbach formalism[Hau 58]. For the emission of a light particle $\left(Z_{1}, A_{1}\right)$ of spin $J_{1}$ from a system $\left(Z_{0}, A_{0}\right)$ of excitation energy $E^{*}$ and spin $J_{0}$, leaving the residual system $\left(Z_{2}, A_{2}\right)$ with spin $J_{2}$, the decay width is given by

$$
\Gamma_{J_{2}}\left(Z_{1}, A_{1}, Z_{2}, A_{2}\right)=\frac{2 J_{1}+1}{2 \pi \rho_{0}} \sum_{1=\mid J_{0}-J_{2} !}^{J_{0}+J_{2}} \int_{0}^{E^{*}-B-E_{T o r}\left(J_{2}\right)} T_{1}(\varepsilon) \rho_{2}\left(U_{2}, J_{2}\right) d \varepsilon
$$

In the above equation $\varepsilon$ and $l$ are the kinetic energy and orbital angular nomentum of the emitted particle, $\rho_{0}$ is the level density of the initial system, $B$ is the binding energy, $E_{r o t}\left(J_{2}\right)$ is the rotation plus deformation energy of the residual system, and $\rho_{2}\left(U_{2}, J_{2}\right)$ is the level density of the residual system with thermal excitation energy

$$
\mathrm{U}_{2}=\mathrm{E}^{*}-\mathrm{B}-\mathrm{E}_{\mathrm{rot}}\left(\mathrm{J}_{2}\right)-\varepsilon
$$


The transmission coefficient $T_{l}(\varepsilon)$ is calculated within the sharp cut-off approximation for a classical system of absorptive radius $\mathbf{R}$, and is equal to

$$
T(\varepsilon)=\begin{gathered}
0 \text { for } \varepsilon<E_{\text {Coul }}+\frac{h^{2} l(l+1)}{2 \mu R^{2}} \\
1 \text { for } \varepsilon>E_{\text {Coul }}+\frac{h^{2} 1(1+1)}{2 \mu R^{2}} .
\end{gathered}
$$

The Coulomb barriers $E_{\text {Coul }}$ are calculated using the empirical expressions of Vaz and Alexander[Vaz 84] and the absorptive radius is equal to $R=1.16 \mathrm{~A}_{2}^{1 / 3}+2.6 \mathrm{fm}$ for proton and neutron emission and $R=1.16 \mathrm{~A}_{2}^{1 / 3}+3.7 \mathrm{fm}$ for alpha particle emission.

The decay width for the emission of heavy fragments $(Z>2)$ is calculated using the transition state formalism of Moretto[Mor 75]. The decay width is given by the following equation:

$$
\Gamma\left(Z_{1}, A_{1}, Z_{2}, A_{2}\right)=\frac{1}{2 \pi \rho_{0}} \quad \int_{0}^{E^{*} \cdot E_{\text {sad }}\left(J_{0}\right)} \rho_{\text {sad }}\left(U_{\text {sad }}, J_{0}\right) d \varepsilon
$$

Here $U_{\text {sad }}$ and $\rho_{\text {sad }}$ are the thermal energy and level density of the conditional saddlepoint configuration. $E_{s a d}\left(J_{0}\right)$ is the deformation plus rotation energy of the saddlepoint configuration and $\varepsilon$ is the kinetic energy of the translational degree of freedom.

The barriers used in these calculations were obtained from the RFRM[Kra 79] using a two spheroid parameterization for the shape of the conditional saddle-point configurations. This parameterization generates conditional barriers which are within 2 MeVof the saddle point energies calculated with more realistic shape parameterizations for $A=110[\mathrm{Dav} 85]$. To correct for this difference, the two-spheroid saddle point energies are scaled by a constant factor for all mass asymmetries and angular 
momentum. The scaling factor is chosen so that for symmetric division the scaled saddle-point energy was equal to the value calculated with the more realistic shape parameterization by Sierk[Sie 86, Dav 85].

The level density is given by the Fermi gas expression[Bet 36, Boh 69]

$$
\rho(\mathrm{U}, \mathrm{J})=(2 \mathrm{~J}+1)\left(\frac{\hbar^{2}}{2 \mathrm{I}}\right)^{3 / 2} \frac{\mathrm{a}^{1 / 2}}{12} \frac{\exp \left(2(\mathrm{aU})^{1 / 2)}\right.}{\mathrm{U}^{2}},
$$

where $I$ is the moment of inertia, $U$ is the thermal energy of the system and a is the level- density parameter. The level-density parameter a is related to the single-particle level density $\mathrm{g}$ by the expression $\mathrm{a}=\frac{\pi^{2} \mathrm{~g}}{6}$ and was given the value $\mathrm{a}=\frac{\mathrm{A}}{8.5} \mathrm{MeV}^{-1}$. 


\section{Footnotes}

1 Within the incomplete fusion model, the dependence of the excitation energy upon source velocity can be derived from kinematical considerations. For instance let $\mathrm{Mp}$ and $\mathrm{Vp}$ be the mass and the velocity of the projectile and let Ms and Vs be the mass and the velocity of the source formed in the incomplete fusion process. Then from conservation of momentum and by neglecting the recoil of the target-like remnant we obtain:

$$
\begin{gathered}
M p V_{p}=M s \text { Vs } \\
1 / 2 M p(V p)^{2} M p=1 / 2 M s(V s)^{2} M s \\
\text { Ep Mp }=\text { Es Ms }
\end{gathered}
$$

where Ep and Es are the total kinetic energy of the projectile and the source respectively. The excitation energy $E^{*}$ deposited in the source can be then calculated from:

$$
E^{*}=E p-E s=E p\left(1-\frac{M p}{M s}\right)=E p\left(1-\frac{V s}{V_{p}}\right)
$$

From the above relation we see that lower values of Vs(more central collisions) correspond to larger values of excitation energy $E^{*}$ and larger values of Vs (more peripheral collisions) are associated with smaller values of $E^{*}$. 
2 Bohr's classical formula gives a reasonable description of the energy loss for heavy particles such as the $\alpha$-particle or heavier nuclei. However for lighter particles, such as the proton, the formula breaks down because of quantum effects. It nevertheless contains all the essential features necessary to describe the energy loss by charged particles due to electronic collisions .

The correct quantum mechanical calculation was first performed by Bethe and Bloch. In the calculation the energy transfer is parametrized in terms of momentum transfer rather than impact parameter. The Bethe-Bloch formula is given by :

$$
-\frac{d E}{d x}=2 \pi N r^{2} m c^{2} \rho \frac{Z}{A} \frac{z^{2}}{\beta^{2}}\left[\ln \left(\frac{2 \gamma^{2} m v^{2} W}{I^{2}}\right)-2 \beta^{2}\right]
$$

with
$\mathrm{N}:$ Avogadros number $=6.02210^{23} \mathrm{~mol}^{-1}$
$\mathrm{Z}$ : atomic number of absorbing
$\rho$ : density of absorbing material material
$z:$ atomic number of incident particle
$\mathrm{r}$ : classical electron radius
e : elementary charge
$\mathrm{m}$ : electron mass
$\mathrm{v}$ : velocity of particle
$\gamma: 1 / \sqrt{1-\beta^{2}}$
$\omega:$ mean orbital frequency of electrons
$\beta: v / c$ of incident particle
I : mean excitation potential
$\mathrm{W}$ : maximum energy transfer in
A: atomic weight of absorbing material
a single collision

3 Angular distributions can be shown in terms of $d \sigma / d \Omega$ or $d \sigma / d \theta$. Isotropic distributions in the reaction plane mean that $d \sigma / d \theta=$ constant and are equivalent to $\mathrm{d} \sigma / \mathrm{d} \Omega \propto 1 / \sin \theta$, which is the equilibrium distribution of fragments emitted from 
systems with high angular momentum. In this work angular distributions will be understood in terms of $\mathrm{d} \sigma / \mathrm{d} \theta$ unless otherwise stated.

4 The emission velocity of a fragment emitted in a compound binary decay (in the center-of-mass) is approximately given by :

$$
V_{1}=\sqrt{\frac{2\left(M_{2}\right) E_{\text {coul }}}{M_{s} M_{1}}}
$$

where $M_{1}$ and $M_{2}$ are the masses of the fragment and its partner, respectively, and $E_{\text {coul }}$ is the Coulomb energy between the two fragments at scission. Assuming that $\mathrm{E}_{\text {coul }} \propto$ $\mathrm{Z}_{1} \mathrm{Z}_{2}$ and $\mathrm{Z} \propto \mathrm{M}$ the above equation may be simplified to

$$
\mathrm{V}_{1} \propto \mathrm{Z}_{\mathrm{S}}-\mathrm{Z}_{1}
$$

where $Z_{S}=Z_{1}+Z_{2}$ is the atomic charge of the composite system. Therefore as $Z_{1}$ increases, $\mathrm{V}_{1}$ becomes progressively smaller. 


\section{Figure Captions}

\section{Chapter 1}

Figure 1.1 Dependence of the total integrated cross section on the center-of-mass energy for emission of complex fragments from the reaction $\mathrm{Cu}+\mathrm{C}$. The points and error bars correspond to the experimental cross sections and statistical errors. The lines are calculations with the statistical model.

Figure 1.2 A diagram illustrating the approximate domains of the various nuclear decay processes.

Figure 1.3 Systematics of Gaussian source radii extracted for a variety of reactions [Zhu 91].

Figure 1.4 Apparent emission temperatures for Ar induced reactions on Au at 60 $\mathrm{MeV} / \mathrm{A}$. The histogram shows the result of a quantum statistical calculation which includes the feeding by sequential decay [Poc 87].

\section{Chapter 2}

Figure 2.1 Schematic ridge-line potentials (solid curves)and expected yields (dashed curves) as a function of the mass-asymmetry coordinate for a) a heavy system above and b) a light system below the Businaro-Gallone point.

Figure 2.2 Schematic potential-energy surface as a function of the reaction and mass-asymmetry coordinates.

Figure 2.3 Comparison of experimental and calculated(GEMINI) charge distributions at six bombarding energies for the $\mathrm{Cu}+\mathrm{C}$ reactions. The experimental 
data are indicated by the diamonds with their respective statistical error. The calculated values are shown by the solid line.

Figure 2.4 Pressure versus density isotherms calculated with a Skyrme force for nuclear matter. The spinodal region is indicated by the dashed lines. The heavy line shows the liquid-gas coexistence region [Sau 76].

Figure 2.5 The relative probability of evaporation, binary fission, cracking and vaporisation mechanisms responsible for the fragment yield as a function of excitation energy of the ${ }^{238} \mathrm{U}$ nucleus, calculated using the microcanonical multifragmentation model of Gross et al.[Gro 87]

Figure 2.6 BNV calculations for a head-on collision $(b=0)$ of the $55 \mathrm{MeV} / \mathrm{a} \mathrm{Mo}+$ Mo reaction at time steps of (a) 20, (b) 60, (c) 120, and (d) $180 \mathrm{fm} / \mathrm{c}$. The front and side views of the colliding systems are given in columns 1 and 2 respectively for a value of the incompressibility constant $\mathrm{K}=200 \mathrm{MeV}$ [Mo 92].

\section{Chapter 3}

Figure 3.1 Schematic representation of the reverse kinematics production of a compound nucleus and its decay by fragment emission.

Figure 3.2 Schematic representation of the experimental setup.

Figure 3.3 Photograph of the detector(Si-array) confuguration.

Figure 3.4 Shematic drawing of the 300 and $5000 \mu \mathrm{m}$ Si detectors. The thick curved line represents the plastic(Lexene) strip on which the telescopes were mounted and illustrates how the telescopes overlapped in order to minimize the dead areas.

Figure 3.5 Schematic representation of the neutron calorimeter.

Figure 3.6 Electronics diagram for the experiment .

\section{Chapter 4}


Figure 4.1 Raw position spectra for a $300 \mu \mathrm{m} \mathrm{Si} \mathrm{detector(top)} \mathrm{and} \mathrm{a} 5000 \mu \mathrm{m}$ detector(bottom). In both cases the Energy signal (electrons) is plotted versus the (Position $x$ Energy)signal(holes). The 15 diagonal lines correspond to the 15 discrete position elements of the devices.

Figure 4.2 Calibrated position spectra from the raw data shown in Figure 4.1.

Figure 4.3 Typical raw ADC energy spectra of the $55 \mathrm{MeV} / \mathrm{A}$ coctail beam in a $300 \mu \mathrm{m}$ Si detector(top) and a $5000 \mu \mathrm{m}$ detector(bottom).

Figure 4.4 Uncorrected(top) and corrected(bottom) energy spectra.

Figure 4.5 Density plots of $\triangle E$ vs $E$ for the reaction $60 \mathrm{MeV} / \mathrm{A} \mathrm{Au}+\mathrm{C}$ for fragments detected at forward laboratory angles.

Figure 4.6 Particle identification spectra for the reaction $60 \mathrm{MeV} / \mathrm{A} \mathrm{Au}+\mathrm{C}$ shown for a telescope near(top) and far(bottom) from the beam. The individual peaks correspond to different elements.

Figure 4.7 Particle identification spectra for the quadruplet( coktail) calibration beam.

Figure 4.8 Representative spectrum of the total neutron light output in MeVee obtained from the $60 \mathrm{MeV} \mathrm{Au}+\mathrm{Cu}$ reactions.

Figure 4.9 Light yield distributions for simulated(top) and experimental(bottom) data with neutron $(14 \mathrm{MeV})$ multiplicities of 10,20 and 30 .

Figure 4.10 Average total light output responce vs the average total number of neutrons emitted at each impact parameter a predicted by the simulations for the 60 $\mathrm{MeV} \mathrm{Au}+\mathrm{Cu}$ reactions.

\section{Chapter 5}

Figure 5.1 Schematic representation of the cross-section $\partial^{2} \sigma / \partial \mathrm{V}_{\perp} \partial \mathrm{V}_{\|}$for complex fragments emitted in a particular asymmetric binary decay from a single 
equilabrated source with a well defined source velocity $V_{s} . V_{1}$ and $V_{2}$ are the velocities with which the complex fragments are emitted in the source frame.

Figure 5.2.a Experimental cross section $\partial^{2} \sigma / \partial V_{\perp} \partial V_{\|}$in the $V_{\perp}-V_{\|}$plane for representative $\mathrm{Z}$-values between 10 and 54 for the $60 \mathrm{MeV} / \mathrm{A}{ }^{197} \mathrm{Au}+{ }^{12} \mathrm{C}$ reaction.

Figure 5.2.b Experimental cross section $\partial^{2} \sigma / \partial V_{\perp} \partial V_{\|}$in the $V_{\perp}-V_{\|}$plane for representative $\mathrm{Z}$-values between 10 and 54 for the $60 \mathrm{MeV} / \mathrm{A}{ }^{197} \mathrm{Au}+{ }^{27} \mathrm{Al}$ reaction.

Figure 5.2.c Experimental cross section $\partial^{2} \sigma / \partial \mathrm{V}_{\perp} \partial \mathrm{V}_{\|}$in the $\mathrm{V}_{\perp}-\mathrm{V}_{\|}$plane for representative $\mathrm{Z}$-values between 10 and 54 for the $60 \mathrm{MeV} / \mathrm{A}{ }^{197} \mathrm{Au}+51 \mathrm{~V}$ reaction.

Figure 5.2.d Experimental cross section $\partial^{2} \sigma / \partial V_{\perp} \partial V_{\|}$in the $V_{\perp}-V_{\|}$plane for representative $\mathrm{Z}$-values between 10 and 54 for the $60 \mathrm{MeV} / \mathrm{A}{ }^{197} \mathrm{Au}+{ }^{63} \mathrm{Cu}$ reaction.

Figure 5.2.e Experimental cross section $\partial^{2} \sigma / \partial V_{\perp} \partial V_{\|}$in the $V_{\perp}-V_{\|}$plane for representative $\mathrm{Z}$-values between 10 and 54 for the $60 \mathrm{MeV} / \mathrm{A}{ }^{197} \mathrm{Au}+{ }^{197} \mathrm{Au}$ reaction.

Figure 5.3 Average source velocity(normalized to beam velocity) as a function of the charge $\mathrm{Z}$ of the detected fragment for all targets: $\mathrm{C}, \mathrm{Al}, \mathrm{V}, \mathrm{Cu}$ and $\mathrm{Au}$.

Figure 5.4 Predicted emission barriers from the Rotating Finite Range Model for ${ }^{197} \mathrm{Au}$ at zero angular momentum.

Figure 5.5 Average emission velocity(squares) in the source frame, as a function of the charge $(\mathrm{Z})$ of the detected fragment for all targets: $\mathrm{C}, \mathrm{Al}, \mathrm{V}, \mathrm{Cu}$ and $\mathrm{Au}$. For comparison a calculation on the based on the Viola systematics(solid line) is also shown.

Figure 5.6.a Angular distributions $(d \sigma / d \theta)$ in the source frame for representative $\mathrm{Z}$-values from the $60 \mathrm{MeV} / \mathrm{A} \mathrm{Au}+\mathrm{C}$ reactions. The $\mathrm{Z}$ values and normalization factors are indicated for each set of points. The curves are fits to the data. 
Figure 5.6.b Angular distributions $(\mathrm{d} \sigma / \mathrm{d} \theta)$ in the source frame for representative $\mathrm{Z}$-values from the $60 \mathrm{MeV} / \mathrm{A} \mathrm{Au}+\mathrm{Al}$ reactions. The $\mathrm{Z}$ values and normalization factors are indicated for each set of points. The curves are fits to the data.

Figure 5.6.c Angular distributions $(d \sigma / d \theta)$ in the source frame for representative $\mathrm{Z}$-values from the $60 \mathrm{MeV} / \mathrm{A} \mathrm{Au}+\mathrm{V}$ reactions. The $\mathrm{Z}$ values and normalization factors are indicated for each set of points. The curves are fits to the data.

Figure 5.6.d Angular distributions $(\mathrm{d} \sigma / \mathrm{d} \theta)$ in the source frame for representative $\mathrm{Z}$-values from the $60 \mathrm{MeV} / \mathrm{A} \mathrm{Au}+\mathrm{Cu}$ reactions. The $\mathrm{Z}$ values and normalization factors are indicated for each set of points. The curves are fits to the data.

Figure 5.6.e Angular distributions $(d \sigma / d \theta)$ in the source frame for representative $\mathrm{Z}$-values from the $60 \mathrm{MeV} / \mathrm{A} \mathrm{Au}+\mathrm{Au}$ reactions. The $\mathrm{Z}$ values and normalization factors are indicated for each set of points. The curves are fits to the data.

Figure 5.7 Angle integrated cross-sections of products from the $60 \mathrm{MeV} / \mathrm{A}{ }^{197} \mathrm{Au}$ $+{ }^{12} \mathrm{C},{ }^{27} \mathrm{Al},{ }^{51} \mathrm{~V},{ }^{63} \mathrm{Cu}$ and ${ }^{197} \mathrm{Au}$ reactions. In all cases the statistical errors are smaller than the size of the data points.

Figure 5.8.a Charge distributions selected by fragment size(largest, 2nd largest, and so on) for coincidence events (2,3,4and 5-fold) arising in the $\mathrm{Au}+\mathrm{C}$ reactions.

Figure 5.8.b Charge distributions selected by fragment size(largest, 2nd largest, and so on) for coincidence events (2,3,4and 5-fold) arising in the $\mathrm{Au}+\mathrm{Al}$ reactions.

Figure 5.8.c Charge distributions selected by fragment size(largest, 2nd largest, and so on) for coincidence events (2,3,4and 5-fold) arising in the $\mathrm{Au}+\mathrm{V}$ reactions.

Figure 5.8.d Charge distributions selected by fragment size(largest, 2nd largest, and so on) for coincidence events (2,3,4and 5-fold) arising in the $\mathrm{Au}+\mathrm{Cu}$ reactions.

Figure 5.8.e Charge distributions selected by fragment size(largest, 2nd largest, and so on) for coincidence events (2,3,4and 5-fold) arising in the $\mathrm{Au}+\mathrm{Au}$ reactions. 
Figure 5.9.a II) Contour plots of the extracted source velocity (Vs) versus total detected charge $\mathrm{Ztot}$ for 2, 3, 4, and 5-fold events from the $\mathrm{Au}+\mathrm{C}$ reactions. I) Projection of the events in II along the Vs axis. The top and bottom arrows indicate the beam and complete fusion velocities respectively. III) Projection of the events in II along the Ztot axis.

Figure 5.9b Same as in Figure 5.9.a for the $\mathrm{Au}+\mathrm{Al}$ system.

Figure 5.9.c Same as in Figure 5.9.a for the $\mathrm{Au}+\mathrm{V}$ system.

Figure 5.9.d Same as in Figure 5.9.a for the $\mathrm{Au}+\mathrm{Cu}$ system.

Figure 5.9.e Same as in Figure 5.9.a for the Au + Au system.

Figure 5.10 Schematic representation of the effect of light charged-particle evaporation on the correlation between Vs and Ztot. The thick solid curve represents the correlation for the primary fragments. Going from right to left the 3 dashed lines represent cases of low, moderate and high excitation energy, respectively, which corresponds to increasing amounts of light charge particle emission.

Figure 5.11 Total detected charge for the $60 \mathrm{MeV} / \mathrm{A} \mathrm{Au}+\mathrm{C}, \mathrm{Al}, \mathrm{V}, \mathrm{Cu}$ and $\mathrm{Au}$ reactions; row 1, 2-fold events; row 2, 3-fold events; row 3, 4-fold events; row 4, 5-fold events.

Figure 5.12 Source velocity distributions for the $60 \mathrm{MeV} / \mathrm{A} \mathrm{Au}+\mathrm{C}, \mathrm{Al}, \mathrm{V}, \mathrm{Cu}$ and Au reactions; row 1, 2-fold events; row 2, 3-fold events; row 3, 4-fold events; row 4, 5fold events.

Figure 5.13 Linear contour plots of the correlation between $\mathrm{Z}_{1}$ and $\mathrm{Z}_{2}$ for the 2fold coincidence events from the $\mathrm{Au}+\mathrm{C}, \mathrm{Al}, \mathrm{V}, \mathrm{Cu}$ and $\mathrm{Au}$ reactions. The distributions have been symmetrized by randomly assigning $Z_{1}$ and $Z_{2}$.

Figure 5.14 Proportion of 2-, 3-, 4-, and 5-fold events as a function of excitation energy per nucleon for the different targets(symbols, see inset) studied at $60 \mathrm{MeV} / \mathrm{A}$. 
Figure 5.15 Corrected(for detector efficiency) proportion of 2-, 3-, 4-, and 5fold events as a function of excitation energy per nucleon for $\mathrm{Au}+\mathrm{Cu}$ at $60 \mathrm{MeV}$.

Figure 5.16a Charge distributions for different gates on the source velosity for the $\mathrm{Au}+\mathrm{C}$ reactions. The number written in each frame is the excitation energy per nucleon calculated from the average source velocity by means of equation 5.5 .

Figure 5.16b Same as in Figure 5.16.a for the $\mathrm{Au}+\mathrm{Cu}$ system.

Figure 5.17 Proportion of complex fragments as a function of excitation energy per nucleon for $\mathrm{Au}+\mathrm{Cu}$ at $60 \mathrm{MeV}$.

Figure 5.18 Neutron multiplicity distributions for the $60 \mathrm{MeV} / \mathrm{A} \mathrm{Au}+\mathrm{Al}, \mathrm{V}, \mathrm{Cu}$ and $A u$ reactions.

Figure 5.19 Neutron multiplicity distribution for $\mathrm{Pb}+\mathrm{Au}$ at $29 \mathrm{MeV} / \mathrm{A}[\mathrm{Pi}$ 91].

Figure 5.20 Distribution of reaction products as gated by contiguous neutron multiplicity bins for the $\mathrm{Au}+\mathrm{Cu}$ reactions at $60 \mathrm{MeV} / \mathrm{A}$.

Figure 5.21 Proportion of 2-, 3-, 4-, and 5-fold events as a function of neutron multiplicity for the different targets studied at $60 \mathrm{MeV} / \mathrm{A}$.

Figure 5.22 Neutron multiplicity versus excitation energy for the different targets studied at $60 \mathrm{MeV} / \mathrm{A}$. The excitation energy was determined by means of equation F.2.

Figure 5.23 Average neutron multiplicity versus excitation energy for the different targets studied at $60 \mathrm{MeV} / \mathrm{A}$. The excitation energy was determined by means of equation F.2.

Figure 5.24 Neutron multiplicity versus excitation energy for several reaction systems in the fissility range $x=0.82-0.91$ [Kno 92].

\section{Chapter 6}

Figure 6.1 $\mathrm{Au}+\mathrm{Cu}$ collisions at $60 \mathrm{MeV} / \mathrm{A}$ calculated with the Landau-Vlasov equation for several impact parameters. The evolution of the reaction can be seen in 
both, the $\mathrm{X}-\mathrm{Y}$ (top) and $\mathrm{Y}-\mathrm{Z}$ (bottom) reaction planes. The time increments are in steps of $20 \mathrm{fm} / \mathrm{c}$.

Figure 6.2 Scatter plot of the evolution of the $\mathrm{Au}+\mathrm{Al}$ collision at $\mathrm{b}=0$ as predicted by the Landau-Vlasov calculations.

Figure 6.3 Time dependence of the emitted nucleon mean velocity in the center of mass as predicted by the Landau-Vlasov calculations for the different targets studied at impact parameter $b=3$.

Figure 6.4 Comparison of the experimentally determined(squares) and calculated(Xs) inclusive cross sections for the $60 \mathrm{MeV} / \mathrm{A} \mathrm{Au}+\mathrm{C}, \mathrm{Al}$, Vand $\mathrm{Au}$ reactions.

Figure 6.5 Comparison of the experimental(solid lines) and calculated(dashed lines) total charge and source velocity distributions for different fragment multiplicities in the case of the $60 \mathrm{MeV} \mathrm{Au}+\mathrm{Cu}$ reactions. The spectra have been normalized to the same maximum. The rightmost arrow corresponds to the beam velocity while the arrow on the left to the complete fusion velocity.

Figure 6.6 The average $Z_{\max }$ as a function of $Z_{\text {tolal }}$ for $A u 60 \mathrm{MeV} / \mathrm{A}$ collisions on $\mathrm{Cu}$. The model calculations(LV + GEMINI) are represented by the line and the experimental data by the points (diainonds).

Figure 6.7 The average multiplicity of IMFs $M_{i m f}$ as a function of $Z_{\text {total }}$ for $A u 60$ $\mathrm{MeV} / \mathrm{A}$ collisions on $\mathrm{Cu}$. The points are the experimental data and the line is the Landau-Vlasov + Gemini prediction.

Figure 6.8 The average value of the relative asymmetry for the two largest fragments as a function of $Z_{\text {total }}$ for $\mathrm{Au} 60 \mathrm{MeV} / \mathrm{A}$ collisions on $\mathrm{Cu}$. The points are the experimental data and the line is the Landau-Vlasov + Gemini prediction. 
Figure 6.9 The average value of the three-body asymmetry $\langle a 3\rangle$ as a function of $\mathrm{Z}_{\text {total }}$ for $\mathrm{Au} 60 \mathrm{MeV} / \mathrm{A}$ collisions on $\mathrm{Cu}$. The points are the experimental data and the line is the Landau-Vlasov + Gemini prediction.

Figure 6.10 Systemtics of the $\tau$ parameter. The reactions and the references are indicated[Tra 92].

Figure 6.11 Single event moments $\mathrm{M}_{3}$ plotted against $\mathrm{M}_{2}$ for the $60 \mathrm{MeV} / \mathrm{A} \mathrm{Au}+$ $\mathrm{Cu}$ reactions.

Figure 6.12 The coincidence charge distribution from which the moments $M_{2}$ and $\mathrm{M}_{3}$ were determined. The line is a best fit to the data

Figure 6.13 Contour plots of $\ln \left(\mathrm{Z}_{\max }\right)$ versus $\ln \left(\mathrm{M}_{2} / \mathrm{M}_{1}\right)$ for different values of the excitation energy. The numbers in each of the subplots represent the excitation energy per nucleon of the events. The upper-leftmost subplot includes all events.

Figure 6.14 The average value of $\ln (\mathrm{M} 2 / \mathrm{M} 1)$ as a function of $\ln (\mathrm{Zmax})$ for 60 $\mathrm{MeV} / \mathrm{A}$ Au collisions on $\mathrm{Cu}$.

Figure 6.15 The average value of $\gamma_{2}$ as a function of $Z_{t o t a l}$ for $A u 60 \mathrm{MeV} / \mathrm{A}$ collisions on $\mathrm{Cu}$. The points are the experimental data and the line is the LandauVlasov + Gemini prediction.

Figure 6.16 a) The fission probability plotted as a function of $E^{-1 / 2}$ for the $\alpha$ induced reactions ${ }^{206} \mathrm{~Pb}(\alpha, f),{ }^{197} \mathrm{Au}(\alpha, f)$, and ${ }^{184} \mathrm{~W}(\alpha, f)$ and $\left.b\right)$ for the electroninduced reactions ${ }^{209} \mathrm{Bi}(\mathrm{e}, \mathrm{f}),{ }^{208} \mathrm{~Pb}(\mathrm{e}, \mathrm{f}),{ }^{174} \mathrm{Yb}(\mathrm{e}, \mathrm{f})$, and ${ }^{154} \mathrm{Sm}(\mathrm{e}, \mathrm{f})[\mathrm{Mor} 69]$.

Figure 6.17 The natural logarithm of the ratio of the 3, 4, and 5-fold to the 2-fold probability(symbols) as a function of $\mathrm{E}^{-1 / 2}$ for the $60 \mathrm{MeV} \mathrm{Au}+\mathrm{Al}, \mathrm{V}$, and $\mathrm{Cu}$ reactions. The lines are best fits to the data. 


\section{References}

Aic 84 J. Aichelin and J. Hufner, Phys. Lett. 136B 15 (1984)

Ai 87 J. Aichelin et al., 581926 (1987)

Aic 91 J. Aichelin, Phys. Rep. 202233 (1991)

Aug 87 F. Auger et al., Phys. Rev. C35 190 (1987)

Bau 85 W. Bauer et al., Phys. Lett. B150 53 (1985)

Bau 86 W. Bauer et al., Nucl. Phys. A452 699 (1986)

Bau 92 W. Bauer et al., Annu. Rev. Nucl. Sci. 4277 (1992) and references therein

Bau 92a W. Bauer et. al., Phys. Rev. Lett. 611988 (1992)

Bal 91 S.P. Baldwin et al., Studies of Heavy-Ion Reactions and Transuranic Nuclei, Progr. Rep., University of Rochester, NY(1991) pp. 151

DOE/ER/40414-4

Bar 86 C. Barbagallo et al., Z. Phys. A324 97 (1986)

Bar 80 M. Barranco et al., Phys. Rev. C22 1729 (1980)

Bar 81 M. Barranco et al., Phys. Rev. C24 1191 (1981)

Bar 86a J. Bartke, Phys. Lett. B174 32 (1986)

Bau 93 E. Bauge et al., Phys. Rev. Lett. 703705 (1993)

Bea 87 G. E. Beauvais et al., Phys. Rev. C35 545 (1987)

Be 36 H.A. Bethe, Phys. Rev. 50332 (1936)

Ber 83 G.F. Bertch and P.J. Siemens, Phys. Lett. 126B 9 (1983) 
Ber 84 G.F. Bertch et. al., Phys. Rev. C29 673 (1984)

Ber 88 G.F. Bertch and S. Das Gupta, Phys. Rep. 160190 (1988); and references contained therein.

Bl 91 Y. Blumenfeld et al., Phys. Rev. Lett. 66576 (1991)

Bo 69 A. Bohr and B. Mottleson, Nuclear Structure, Volume 1, Benjamin NY

Boa 83 D.H. Boal, Phys. Rev. C28 2568 (1983)

Boa 86 D.H. Boal et al., Phys. Rev. Lett. 572901 (1986)

Boa 88 D.H. Boal and J.N. Glosli, Phys. Rev. C37 91 (1988)

Boh 39 N. Bohr and J.A. Wheeler, Phys. Rev 56426 (1939)

Boh 83 S. Bohrmann et al., Phys. Lett. B120 59 (1983)

Bou 89 R. Bougault et al., Phys. Lett. B232 291 (1989)

Bou 88 R. Bougault et al., Nucl. Phys. A488 255c (1988)

Bo 84 B. Borderie et al., Z. Phys. A318 315 (1984)

Bor 90 B. Borderie et al., Ann. Phys. Fr. 15287 (1990) and references therein

Bor 92 B. Borderie et al., Phys. Lett. B302 15 (1992)

Bor 88 B. Borderie et al., Phys. Lett. B205 26 (1988)

Bo 89 A. Bonasara et al., Phys. Lett. B 221233 (1989)

Bo 90a A. Bonasara et al., Phys. Lett. B 244169 (1990)

Bo 90b A. Bonasara tt al., Phys. Lett. B 246337 (1990)

Bon 83 J.P. Bondorf et al., Nucl. Phys. A443 321 (1983)

Bon 85 J.P. Bondorf et al., Nucl. Phys. A444 460 (1985)

Bon 90 J.P. Bondorf et al., Phys. Lett. B240 28 (1990)

Bow 91 D. R. Bowman et al., Nucl. Phys. A523 386(1991)

Bow 91b D. R. Bowman et al., Phys. Rev. Lett. 671527 (1991)

Bow 92 D. R. Bowman et al., Phys. Rev. C46 1834 (1992)

Bra 84 M. Brack, Phys. Rev. Lett. 53119 (1984) 
Bu 92 G.F. Burgio et al., Phys. Rev. Lett. 69885 (1992)

Bu 55 U.L. Businaro and S. Gallone, Nuovo Cimento 1629 (1955) and 11277

Cam 88 X. Campi, Phys. Lett. B208 351 (1988)

Cam 86 X. Campi, J. Phys. A. 19 L917 (1986)

Ca 58 A. A. Caretto et al., Phys. Rev. 110, 1130 (1958)

Ca 88 W. Cassing and U. Mosel, Prog. Part. Nucl. Phys. 25235 (1988)

Ceb 89 D. A. Cebra et al., Phys. Lett. B227 336 (1989)

Ceb 90 D.A. Cebra et al., Phys. Rev. Lett 642246 (1990)

Ch 88a R.J. Charity et. al., Nucl. Phys. A476 516 (1988)

Ch 88b R.J. Charity et. al., Nucl. Phys. A483 371 (1988)

Ch 90 R.J. Charity, Nucl. Phys. A511 59 (1990)

Cha 91 M.B. Chatterjee et al., Studies of Heavy-Ion Reactions and Transuranic Nuclei, Progr. Rep., University of Rochester, NY(1991) pp. 57

DOE/ER/40414-4

Cha 91a A.D. Chacon et. al., Phys. Rev. C43 2679 (1991)

Che 87 Z. Chen et al., Phys .Lett. B186 280 (1987)

Che 88 Z. Chen et al., Phys. Rev. C38 2630 (1988)

Chi 83 C. B. Chitwood et al., Phys. Lett. 131B 289 (1983)

Co 86 S. Cohen, Ann. Phys. A457 441 (1986)

Co 92 M. Colonna et al., Phys. Lett. B283 180 (1992)

Co 89 N. Colonna et. al., Phys. Rev. Lett. 621833 (1989)

Coh 63 S. Cohen et al., W.J.: AS 63 p.325 (1963)

Coh 74 S. Cohen et al., Ann. Phys. (NY) 82557 (1974)

Cug 83 J. Cugnon et al., Nucl. Phys. A339 519 (1983)

Cug 82 J. Cugnon et al., Phys. Lett.. B109 167 (1982) 
Da $85 \quad$ K.T. Davies and A.J. Sierk, Phys Rev. C31 915 (1985) (1969)

Dan 83 P. Danielewicz et al., Phys. Lett. B129 283 (1983)

Dav 78 K.T.R. Davies et al., Phys. Rev. Lett. 41632 (1978)

De 90 D. N. Delis, et. al. .Z. Phys. A339 279 (1990)

De 91 D. N. Delis, et. al. .Nucl. Phys. A534 403 (1991)

DeS 91 R.T. de Souza, et. al., Phys. Lett. B268 6 (1991)

Des 87a J. Desbois et al., Z. Phys. A328 101 (1987)

Des 87b J. Desbois et al., Nucl. Phys. A466 724 (1987)

Era 91 B. Erazmus et al., Phys. Rev C44 2663 (1991)

Fi 86 D.J. Fields et al., Phys. Rev. C34 536 (1986)

Fie 84 D.J. Fields et al., Phys. Rev. C30 1912 (1984)

Fis 67 M.E. Fisher et al., Physics (N.Y) 3255 (1967)

Fin 82 J.E. Finn et al., Phys. Rev. Lett. 491321 (1982)

Fri 54 G. Friedlander et al., Phys. Rev. 94727 (1954)

Fri 83 W.A. Friedman et al., Phys. Rev. C28 16 (1983)

Fri 88 W.A. Friedman et al., Phys. Rev. Lett. 602125 (1988)

Fri 90 W.A. Friedman, Phys. Rev. C42 667 (1990)

Gav 80 A. Gavron, Phys. Rev. C21 230 (1980)

Gal 70 J. Galin et. al., Nucl. Phys. A 159461 (1970) .

Gal 88 J. Galin et al., Z. Phys. A331. 63 (1988)

Go 77 J. Gosset et al., Phys. Rev C16 629 (1977)

Gon 91 W. G. Gong et al., Phys. Rev C43 1804 (1991)

Gon 91a W. G. Gong et. al., Phys. Rev C43 781 (1991)

Goo 84 A.L. Goodman et al., Phys . Rev. C30 851 (1984)

Gos 76 J. Gosset et al., Phys. Rev. C16 629 (1976) 
Gou 91 D. Goujdami et al., Z. Phys. A339 293 (1991)

Gra 86 C.R.Grant, Phys. Rev. C34 1950 (1986)

Gro 82 D.H.E. Gross et al., Z. Phys. A309 41 (1982)

Gro 85 D.H.E. Gross Nucl. Phys. A428 313c (1985)

Gro 82 D.H.E. Gross et al., Phys. Lett. B200 397 (1987)

Gro 87 D.H.E. Gross and H. Massmann, Nucl. Phys. A471 355c (1987)

Gro 90 D.H.E. Gross, Rep. Prog. Phys. 53605 (1990)

Gr 87 C. Gregoire et al., Nucl. Phys. A465 315 (1987)

$\mathrm{Gu} 89$ D. Guerreau, in Proceedings of the International School on Nuclear Physics: Nuclear Matter and Heavy Ion Collisions, Les Houches, France, 1989

Hag 89 K. Hagel et al., Phys. Lett. B229 20 (1989)

Ha 92 K. Hagel et al., Phys. Rev. Lett. 682142 (1992)

Han 89 H.Y. Han et. al. , Nucl. Phys. A492 138 (1989)

Ha 52 H. Hauser and H. Feshbach, Phys. Rev. 87366 (1952)

Han 56 R. Hanbury Brown et al., Nature 17727 (1956)

Hei 88 H. Heiselberg et al., Phys. Rev. Lett. 61818 (1988)

Her 82 H.J. Herrmann et al., Phys. Rev. Lett. 49412 (1982)

Huf 83 J., Hufner et al., Phy. Rev. C27 2090 (1983)

Hub 80 F. Hubert et al., Supp. Ann. Phys. Fr. 51 (1980)

Hy 71 E.K. Hyde et. al., Phys. Rev. C 4, 1795 (1971)

Jac 91 D. Jacquet et al., Studies of Heavy-Ion Reactions and Transuranic Nuclei, Progr. Rep., University of Rochester, NY(1991) pp. 118 DOE/ER/40414-4

Ja 82 B. Jakobson et al., Z. Phys. A307 293 (1982)

Jak 90 B. Jakobson et al., Nucl. Phys. A. 509195 (1990) 
Jak 91 B. Jakobson et al., Phys. Rev. C44 R1238 (1991)

Jaq 91 H. R. Jaqaman et al., Nucl. Phys. A 524321 (1991)

Jia 89 D.X. Jiang et. al., Nucl. Phys. A503 561 (1989)

Jou 91 D. Jouan et al., Z. Phys. A 34063 (1991)

Ji 88 G.M. Jin et al., Proceedings of the 3rd Intern, Conf. on Nucleus-Nucleus Collisions, Saint-Malo, Communications p.77 1988

Kau 59 R. Kaufmann and R. Wolfgang Phys. Rev. Lett 3232 (1959)

Ke 92 W.L. Kehoe et al., Nucl. Instr. and Meth. A311 258 (1992)

Ki 89 Y. D. Kim et al., Phys. Rev. Lett. 63494 (1989)

Kim 92 Y. D. Kim et al., Phys. Rev. C45 387 (1992)

Kno 92 K. Knoche et al., Z. Phys. A342 319 (1992)

Ko 73 Ralph G. Korteling et al., Phys. Rev. C 7, 1611 (1973)

Koo 89 S.E. Koonin et al., Phys. Rev. Lett. 621247 (1989)

Kra 74 J.V. Kratz et. al., Phys. Rev. Lett. 33502 (1974)

Kr 79 H.J. Krappe Phys. Rev. C20 992 (1979)

Kre 92 P. Kreutz et al., GSI-92-23, Gessellschaft fur Schwerionenforschung $\mathrm{mbH}(1992)$

Kun 91 G.J. Kunde et al., GSI-91-17, GSI (1991)

Kya 86 A. Kyanowski et al., Phys. Lett B181 43 (1986)

Lam 81 D.Q. Lamb et al., Nucl. Phys. A360 459 (1981)

Lop $84 \quad$ J.A. Lopez and P.J. Siemens, Nucl. Phys. A431 728 (1984)

Lop 89 J. A. Lopez et al., Nucl. Phys. A491 477 (1989)

Lot 91 B. Lott et al., Studies of Heavy-Ion Reactions and Transuranic Nuclei, Progr. Rep., University of Rochester, NY(1991) pp. 187 DOE/ER/40414-4

Lyn 82 U. Lynen et al., Nucl. Phys. A387 129 (1982) 
Mi 53 J.M. Miller and G. Friedlander, Phys. Rev. 91, 485 (1953)

Min 82 R.W. Minich et al., Phys. Lett. 118B 458 (1982)

Mc 85 M.A. McMahan et al., Phys. Rev. Lett. 541995 (1985)

Mc 86 M.A. McMahan et al., Nucl. Instr. and Meth. A253 1 (1986)

Me 80 W. G. Meyer et al., Phys. Rev. C 22, 179 (1980)

Med 92 E.L. Medeiros et al., J. Phys. Rev. 45C 372 (1991)

Mek 77 A.Z. Mekjian, Phys. Rev. Lett. 38640 (1977)

Mo 69 L.G. Moretto et al., Phys. Rev. 41176 (1969)

Mo 75 L.G. Moretto, Nucl. Phys. A247, 211 (1975) (1955)

Mor 73 L.G. Moretto et. al., Physics and Chemistry of Fission vol. 2 (Vienna:IAEA) p. 351 (1973)

Mor 81 L.G. Moretto and R.P. Schmitt, Reports on Progress in Physics $44533(1981)$

Mor 84 D.J. Morissey et al., Phys. Lett. B148 423 (1984)

Mor 87 L.G. Moretto et al., 8th High Energy Heavy Ion Study, Berkeley, CA 1987, pp. 273-87. LBL

Mor 88 L.G. Moretto et. al., Prog. Part. \& Nucl. Phys. 21401 (1988)

Mo 85 J.J. Molitoris et al., Prog. in Part. and Nucl. Phys. 15239 (1985)

Mor 86 L.G. Moretto and D. R. Bowman, in Proceedings of the 24th International Winter Meeting on Nuclear Physics, Bormio, Italy , 1986 [Ric. Sci. ed. Educazione Permanente, Suppl. 49, 126 (1986)]

Mo 92 L.G. Moretto et al., Phys. Rev. Lett. 691884 (1992)

Mor 88 M. Morjean et al., Phys. Lett. B203 215 (1988)

Nay 92 T.K. Nayak et al., Phys. Rev. C45 1 (1992)

Neg 82 J.W. Negele et al., Rev. Mod. Phys. 54913 (1982) 
Ne 86a J. Nemeth et al., Z. Phys. A325 347 (1986)

Ne 86b J. Nemeth et al., Z. Phys. A323 419 (1986)

Ngo 90 H. Ngo et al., Z. Phys. A337 81 (1990)

Ni 65 J.R. Nix and W.J. Swiatecki, Nucl. Phys. 711 (1965)

Og 91 C.A. Ogilvie et al., Phys. Rev. Lett. 671214 (1991)

Olm 87 A. Olmi et. al., Europhys. Lett. 41221 (1987)

Olm 87a A. Olmi, Nucl. Phys. A471 97c (1987)

Pa 88 A. Pantaleo et al., Nucl. Instr. and Meth. A269 580 (1988)

Pa 90 A. Pantaleo et al., Nucl. Instr. and Meth. A286 230 (1990)

Pe 89 G. Peilert et al., Phys. Rev. C39 1402 (1989)

Pet 87 C.J. Pethic and D.G. Ravenhall Nucl. Phys. A471 19c (1987)

Po 71 A.M. Poskanzer et al., Phys. Rev. C 3, 882 (1971)

Poc 86 J. Pochodzalla et al., Phys. Lett. B174 36 (1986)

Poc 87 J. Pochodzalla et al., Phys. Rev. C35 1965 (1987)

Pl 89 E. Plagnol, et. al., Phys. Lett. B221 11 (1989)

Pi 91 E. Piasecki et. al., Phys. Rev. Lett. 661291 (1991)

Que 91 B. Quednau et al., Studies of Heavy-Ion Reactions and Transuranic Nuclei, Progr. Rep., University of Rochester, NY(1991) pp. 187 DOE/ER/40414-4

Ran 81 J. Randrup and S.F. Koonin, Nucl. Phys. A356 223 (1981)

Riv 88 M.F. Rivet et. al., Phys. Lett. B215 55 (1988)

Rou 93 P. Roussel-Chomaz et. al.,Nucl Phys. A551 508 (1993)

Sau 76 G.H. Sauer et al., Nucl Phys., A264 221 (1976)

Sch 77 W.U. Schroeder and J.R. Huizenga, Annual Review of Nuclear and Particle Science 27465 (1977)

Sch 89 P. Schuck, Prog. Part. Nucl. Phys. 22181 (1981) 
Sch 91 W.U. Schroeder, Studies of Heavy-Ion Reactions and Transuranic Nuclei, Progr. Rep., University of Rochester, NY(1991) pp. 118 DOE/ER/40414-4

Si 86 A.J. Sierk Phys. Rev. C33 2039 (1986)

Si 85 A.J. Sierk Phys. Rev. Lett. 55582 (1985)

Si 83 P.J. Siemens, Nature 305410 (1983)

Sin 89 Y. M. Sinyukov, Nucl. Phys. A498 151c (1989)

Sin 91 Y. M. Sinyukov et al., Z. Phys. C49 417 (1991)

Sn $88 \quad$ K. Sneppen and L. Vinet, Nucl. Phys. A511 414 (1990)

So 83 L. G. Sobotka et al., Phys. Rev. Lett. 512187 (1983)

So 84 L. G. Sobotka et al., Phys. Rev. Lett. 532004 (1984)

Sob 85 L. G. Sobotka et al., Phys. Rev. C31 668 (1985)

St 86 H. Stocker and W. Greiner, Phys. Rep. 137277 (1986)

St 84 R. G. Stokstad, in Treatise on Heavy Ion ScienceVol.3 edited by D.A. Bromley; Plenum: New York, 1984; Chp 2.

Sta 71 H.E. Stanley, Introduction to phase transitions and critical phenomena (Oxford U.P., Oxford, 1971)

Sta 85 D. Stauffer, Introduction to Percolation theory(Taylor and Francis, London, 1985)

Tok 92 J. Toke et. al., Annu. Rev. Nucl. Part. Sci. 42401 (1992)

Tr 87 R. Trockel et al., Phys. Rev. Lett. 592844 (1987)

Tr 89 R. Trockel et al., Phys. Rev. C39 729 (1989)

Tra 92 W. Trautmann et al., GSI-92-44, Gessellschaft fur Schwerionenforschung $\mathrm{mbH}(1992)$

Tsa 85 M. B. Tsang et al., Phys. Rev. C40 1685 (1989)

Tsa 89 M. B. Tsang et al., Phys. Lett. B220 492 (1989) 
Va 84 L. Vaz anf J.M. Alexander, Z. Phys. A318 231 (1984)

Vi 85 V.E. Viola, et. al., Phys. Rev. C 31, 1550 (1985)

Wa 85 C.J. Waddington and P.S. Freiser, Phys. Rev. C31 888 (1985)

Wa 90 J.T. Walton et al., IEEE Trans. Nucl. Sci. NS-37 1578 (1990)

Wa 87 J.T. Walton et al., IEEE Trans. Nucl. Sci. NS-34 396 (1987)

Wa 78 J.T. Walton et al., IEEE Trans. Nucl. Sci. NS-25 391 (1978)

Wei 37 V.F. Weisskopf et al., Phys. Rev. 52295 (1937)

Wes 76 G.D. Westfall et al., Phys. Rev. Lett. 371202 (1976)

Woz 93 G. J. Wozniak et al., to be published

Xu 93 Xu X. M. et al., 93-04, Texas A\&M University (1993)

Zhu 91 F. Thu et al., Phys. Rev. C44 R582 (1991) 


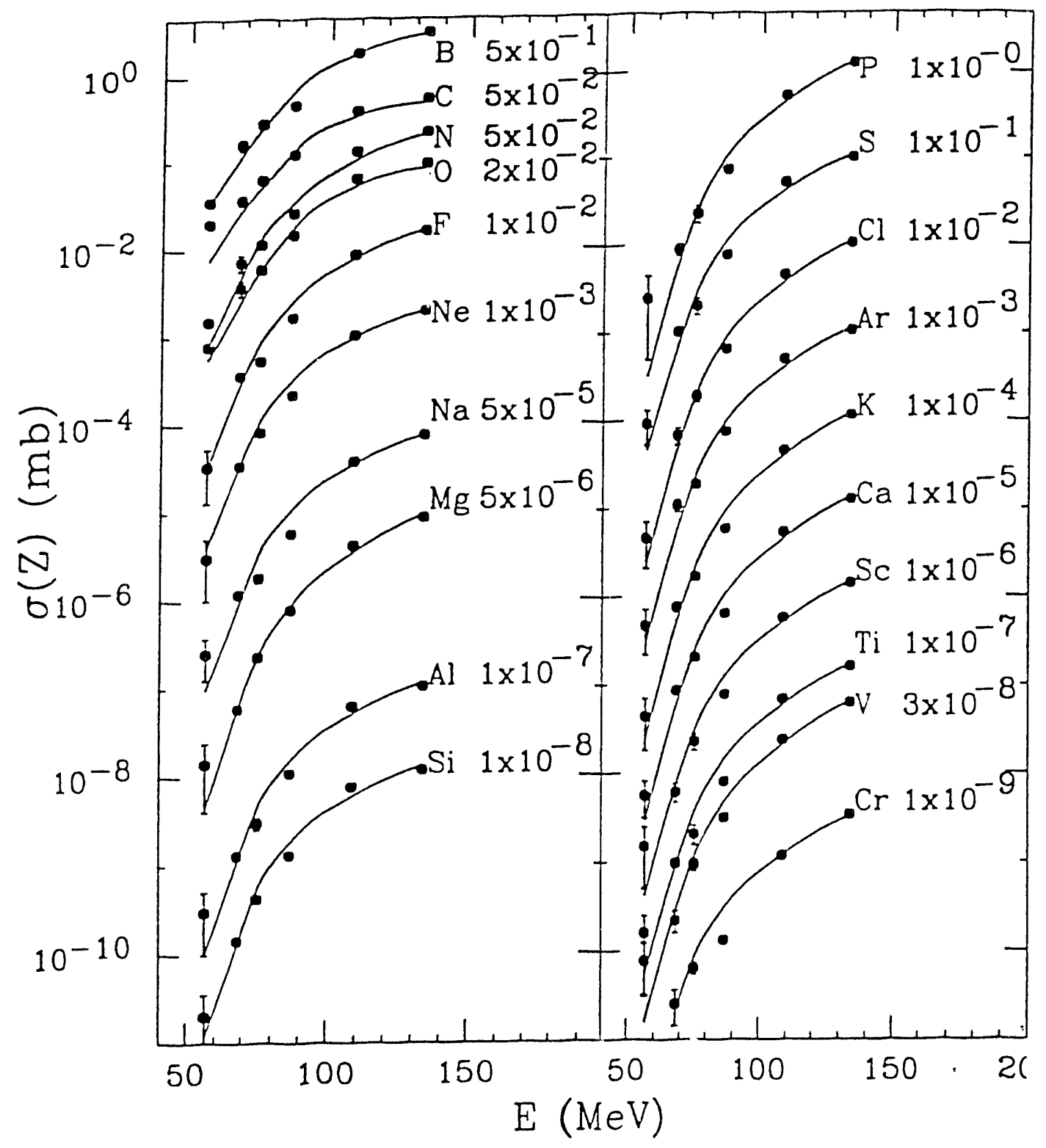

Figure 1.1 


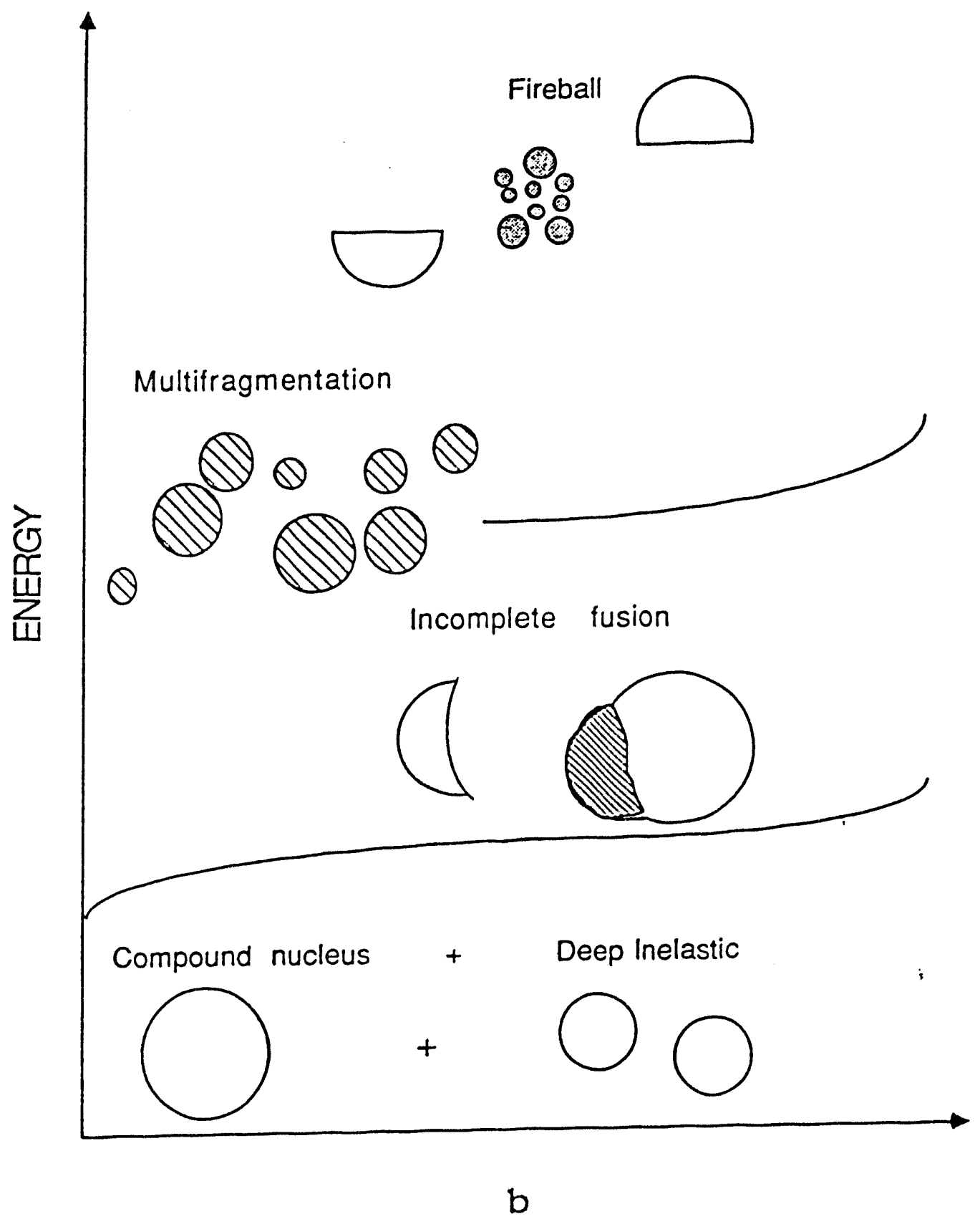

Figure 1.2 


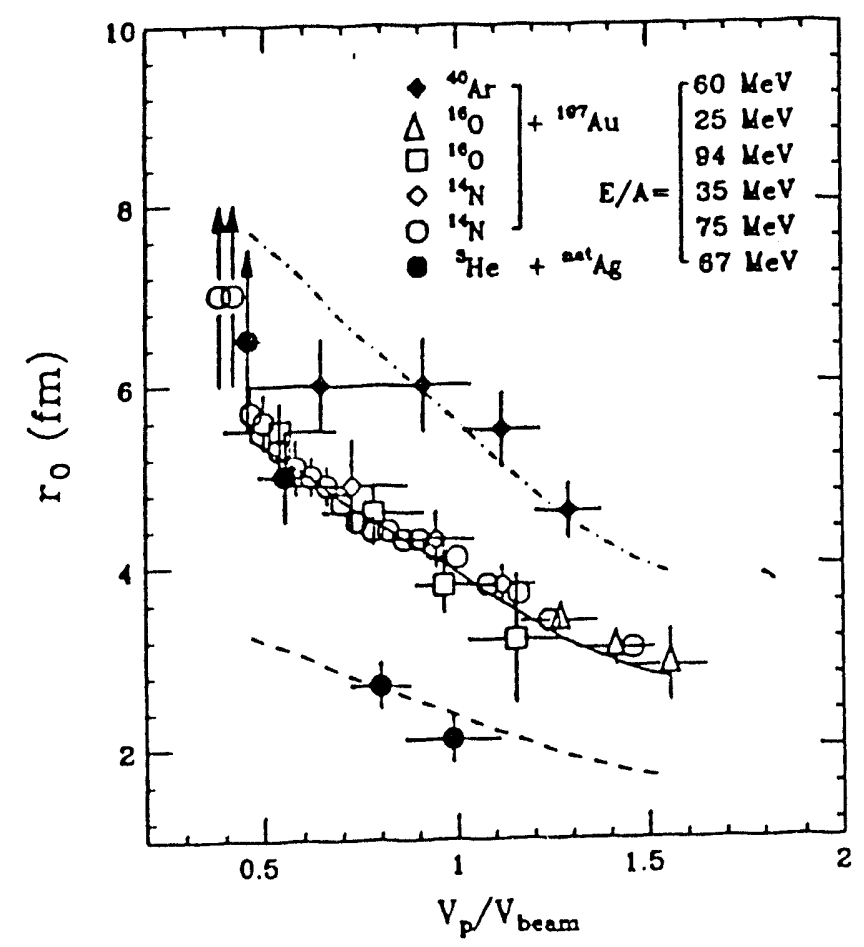

Figure 1.3

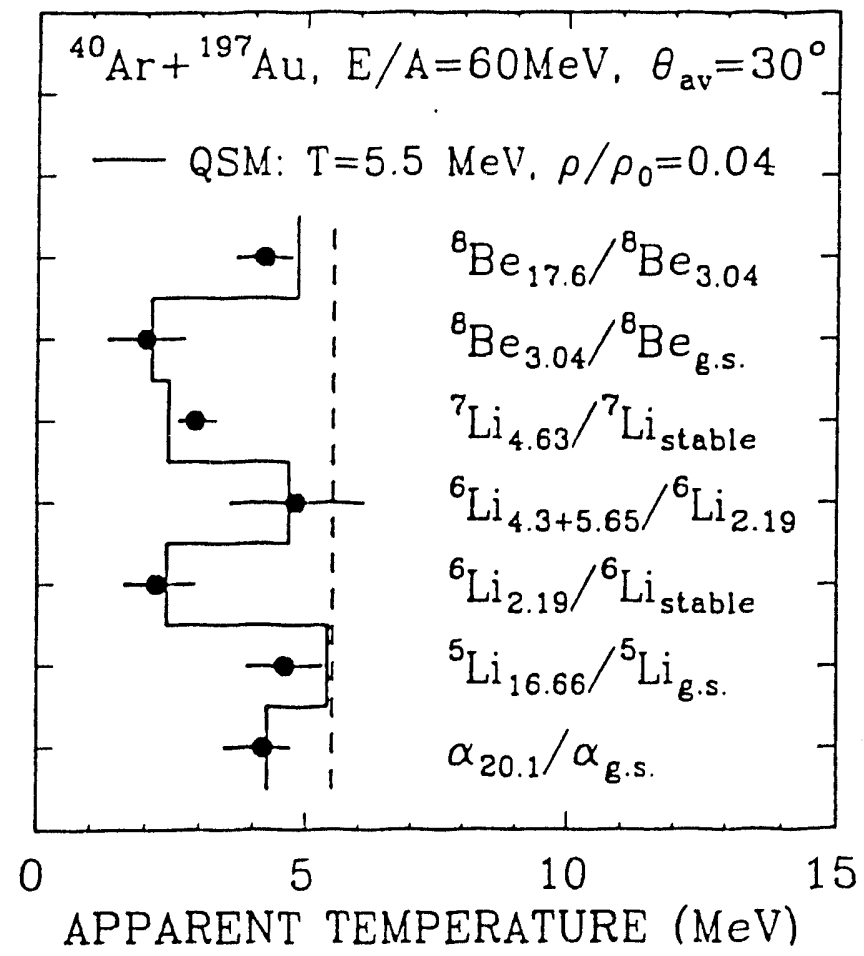

Figure 1.4 


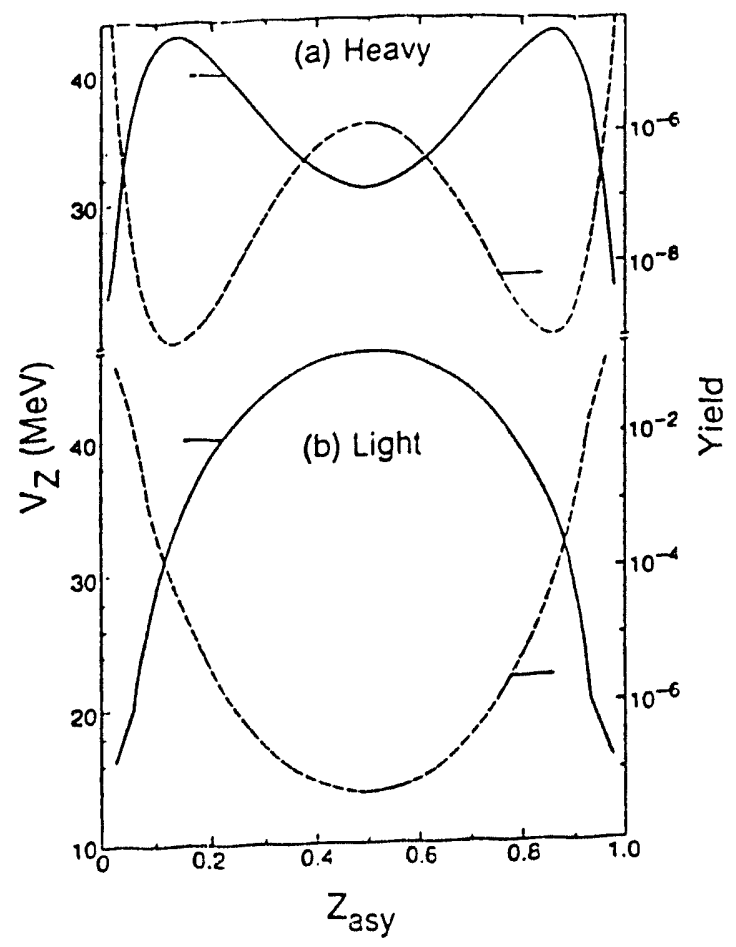

Figure 2.1

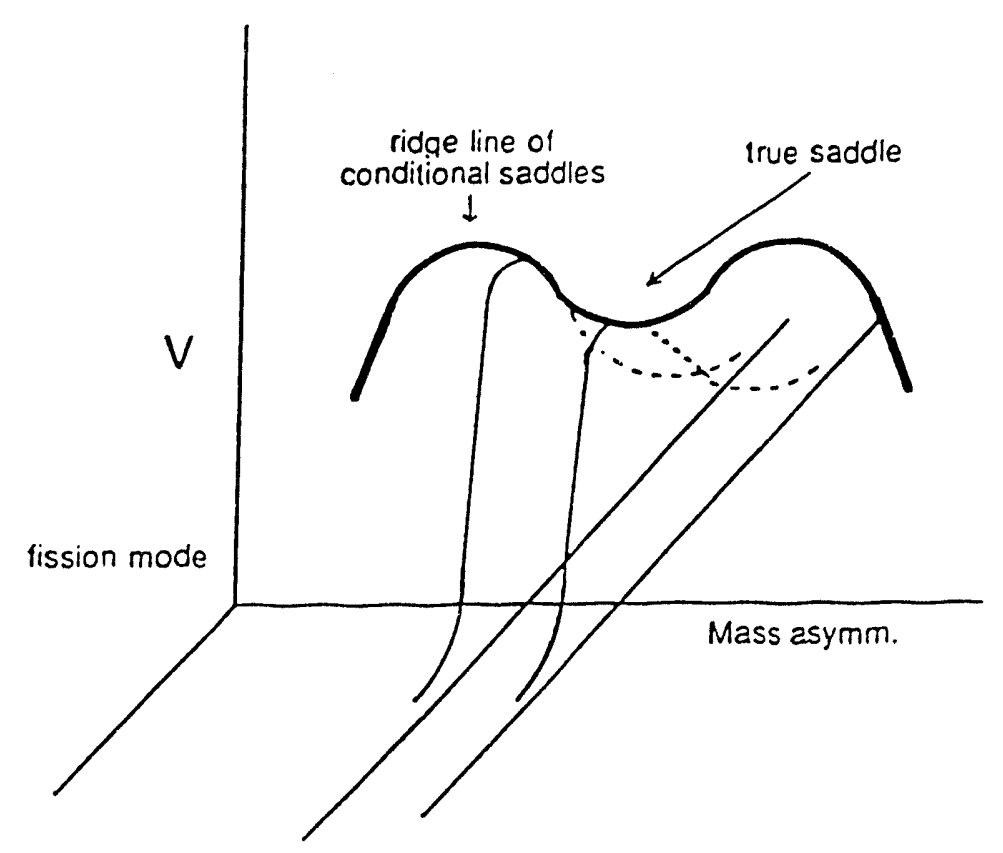

Figure 2.2 


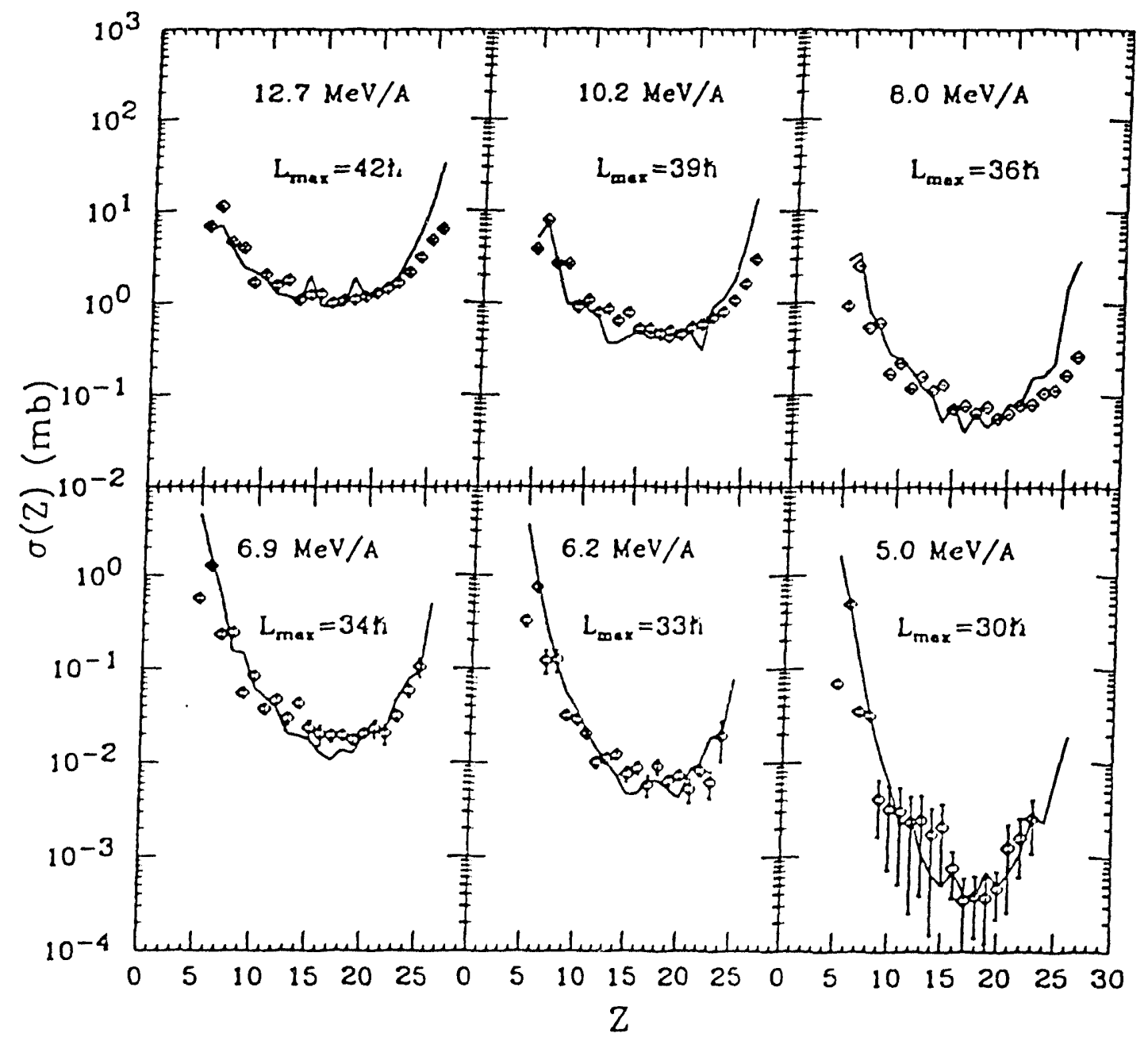

Figure 2.3 


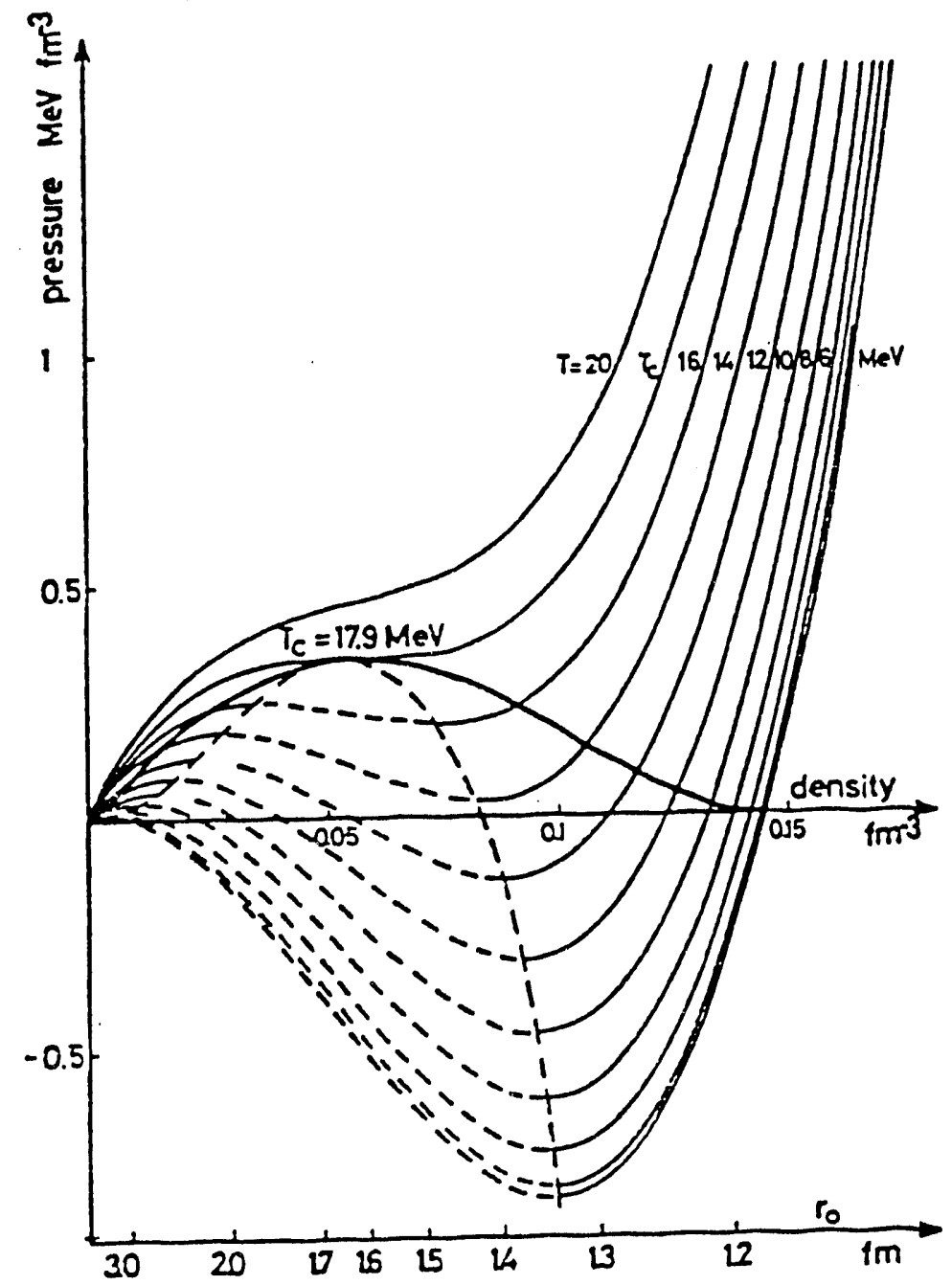

Figure 2.4 


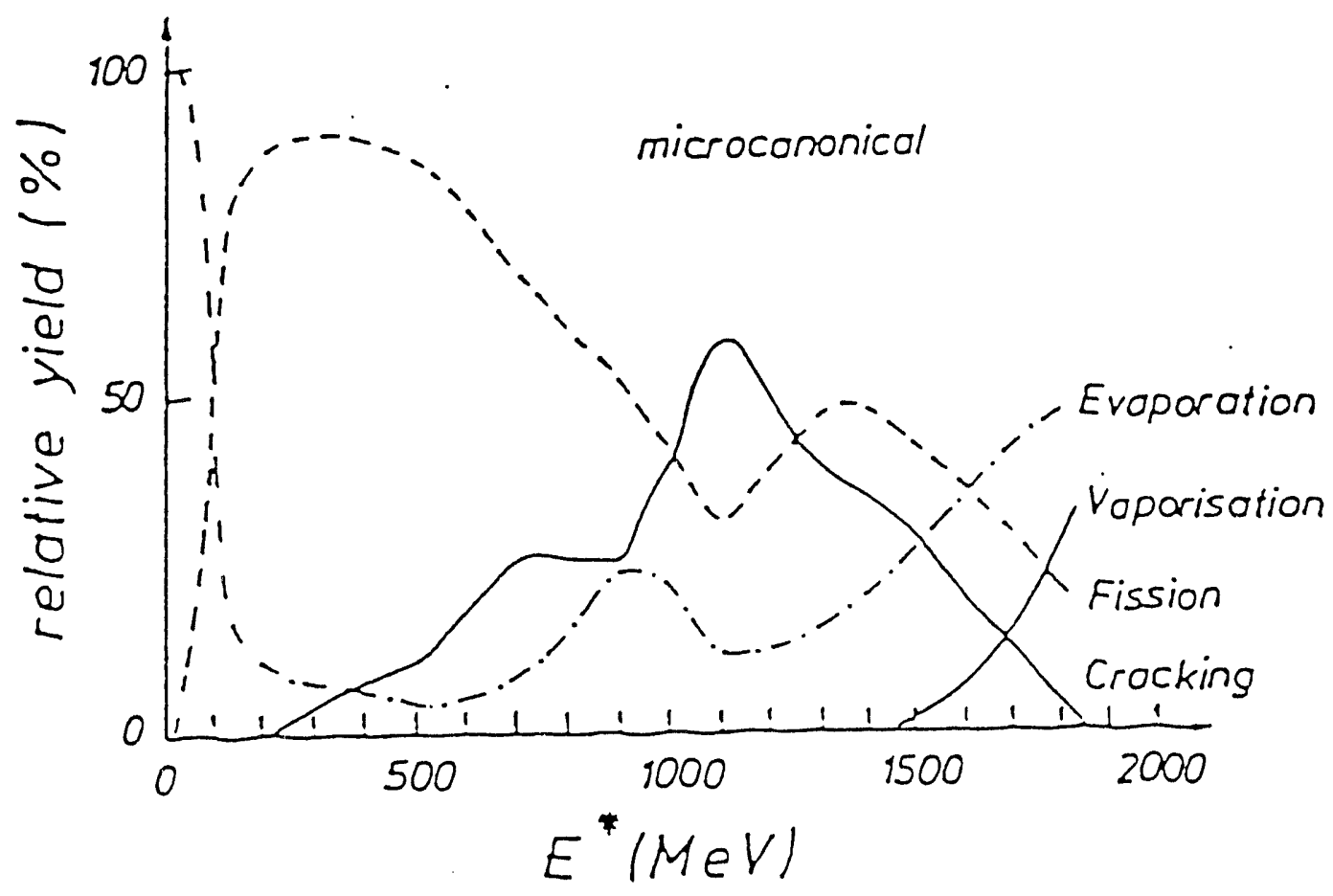

Figure 2.5 
$55 \mathrm{MeV} / \mathrm{u} \mathrm{Mo}+\mathrm{Mo}, \mathrm{b}=0$

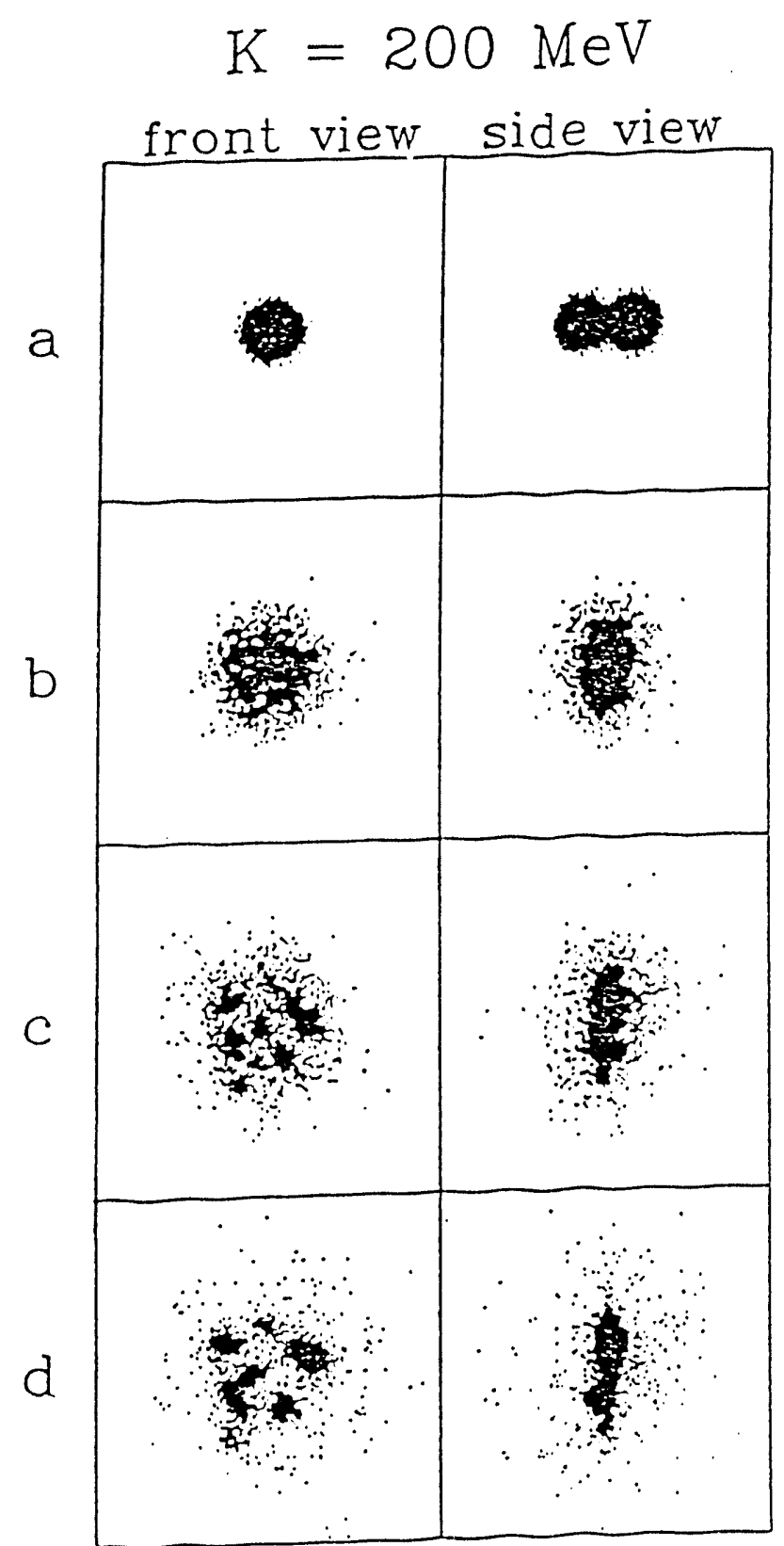

Figure 2.6 


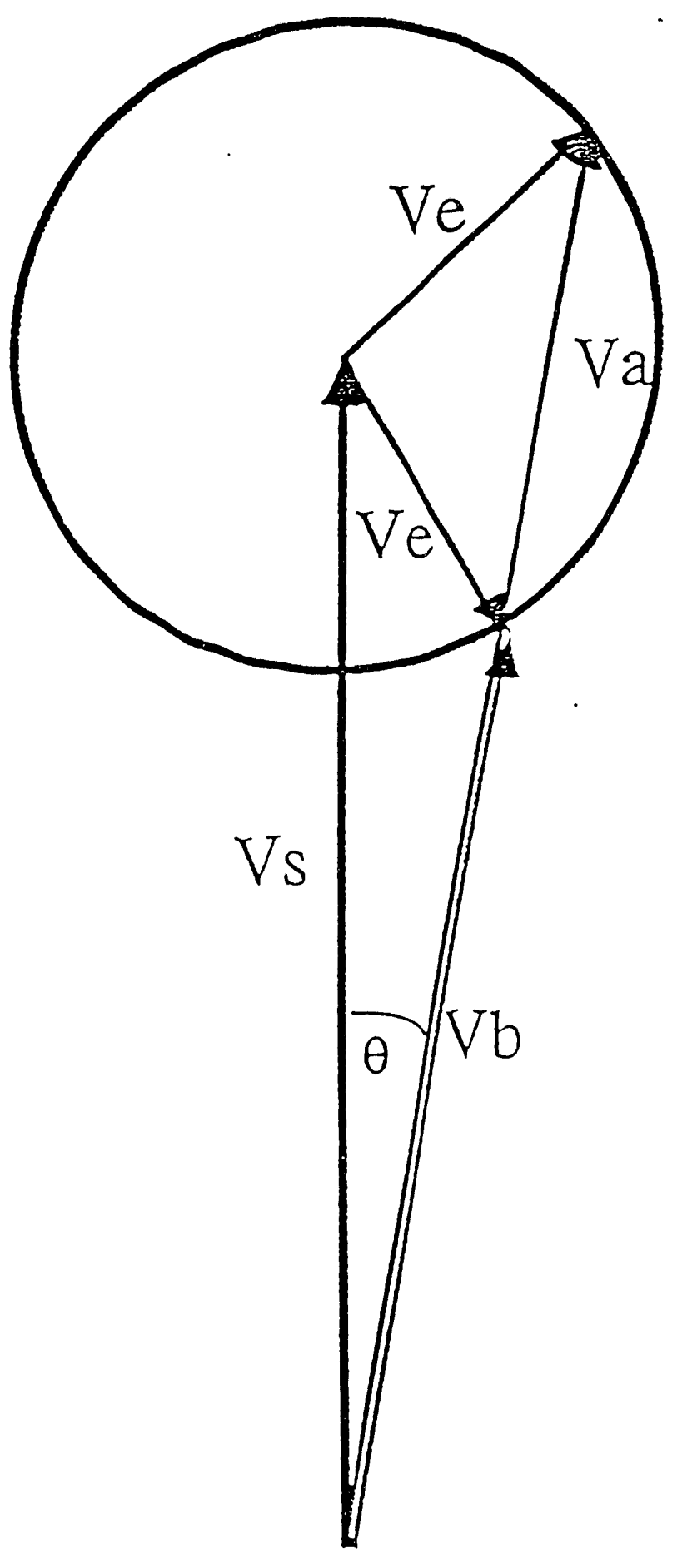

Figure 3.1 


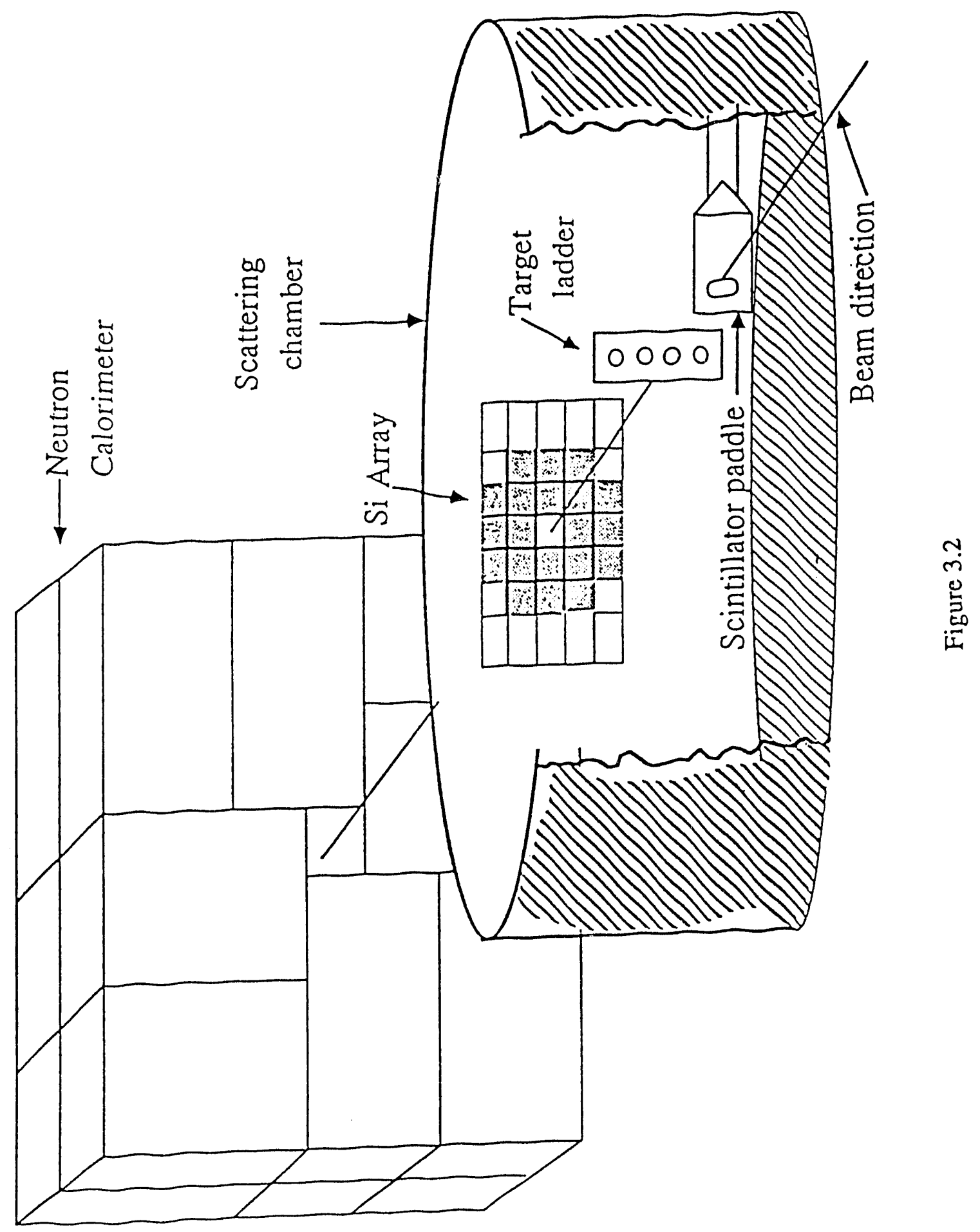




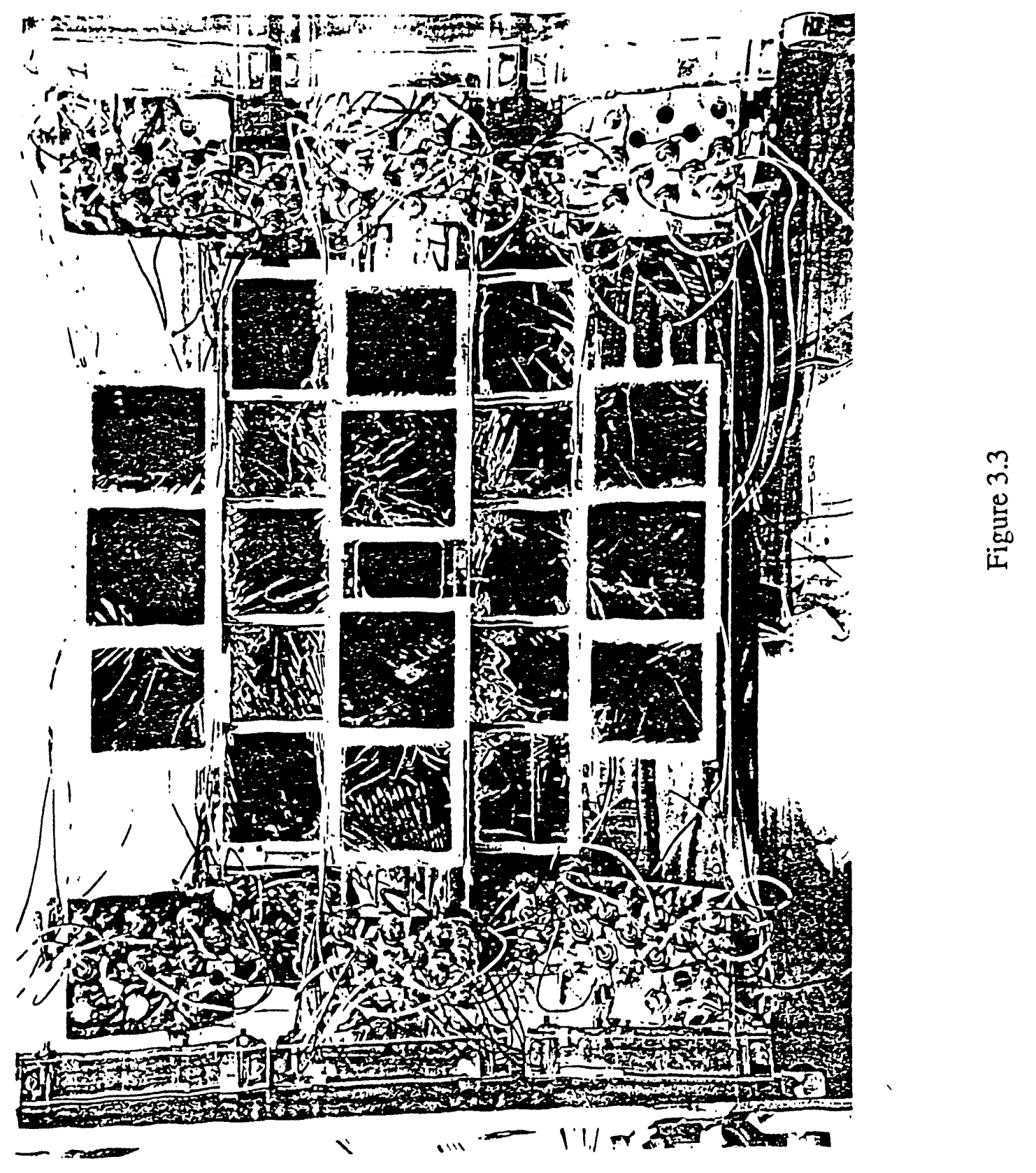




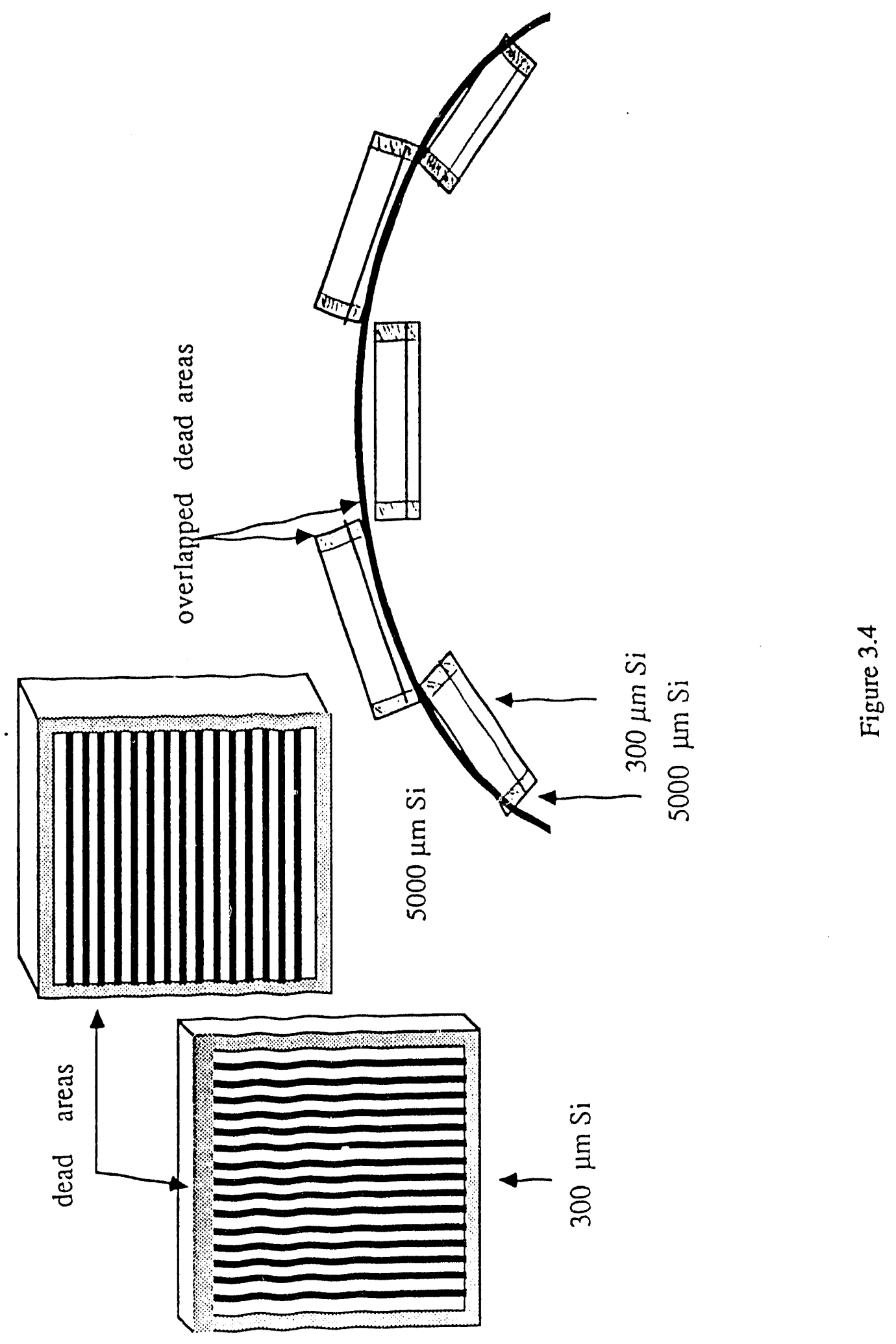




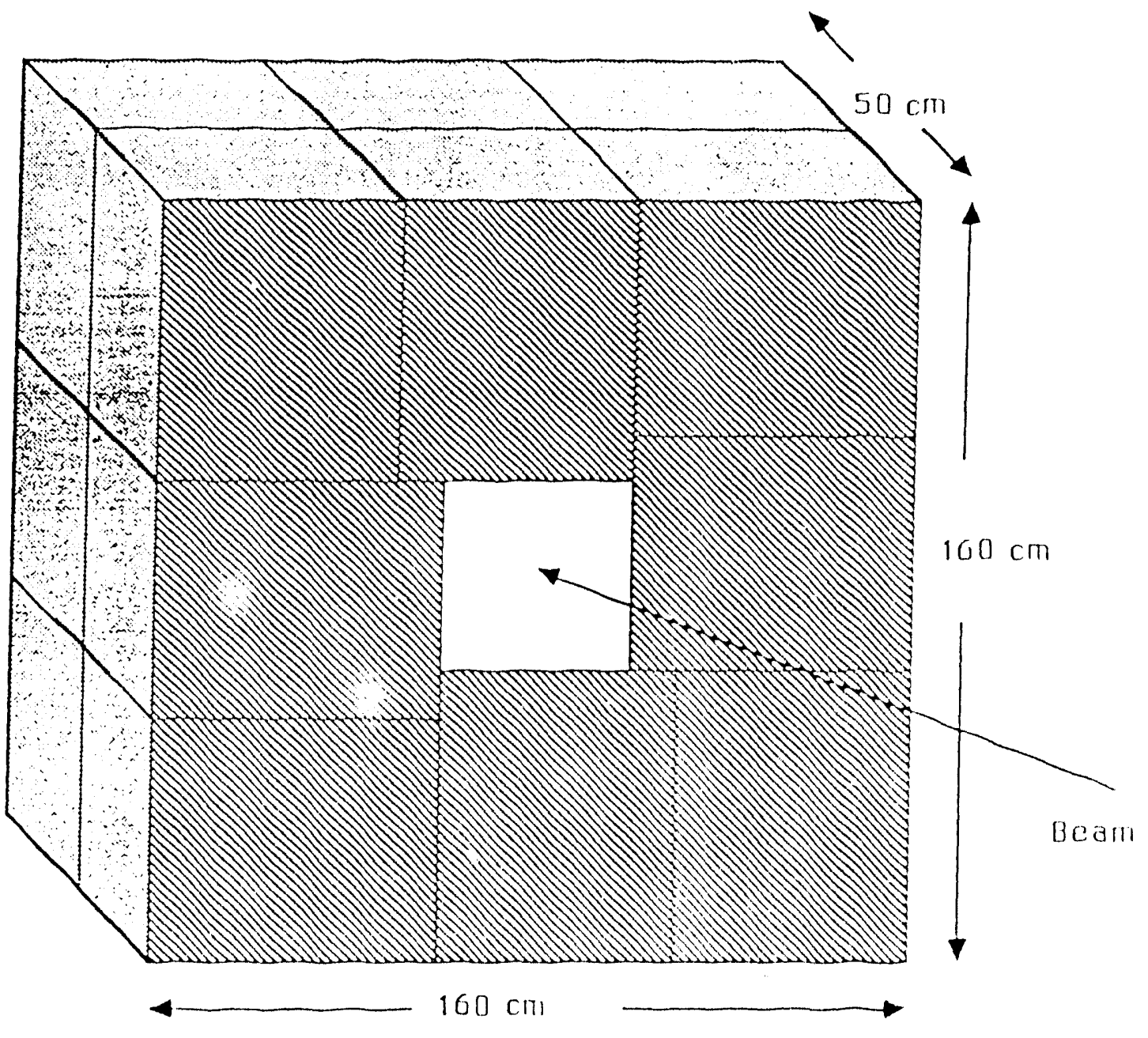

Figure 3.5 

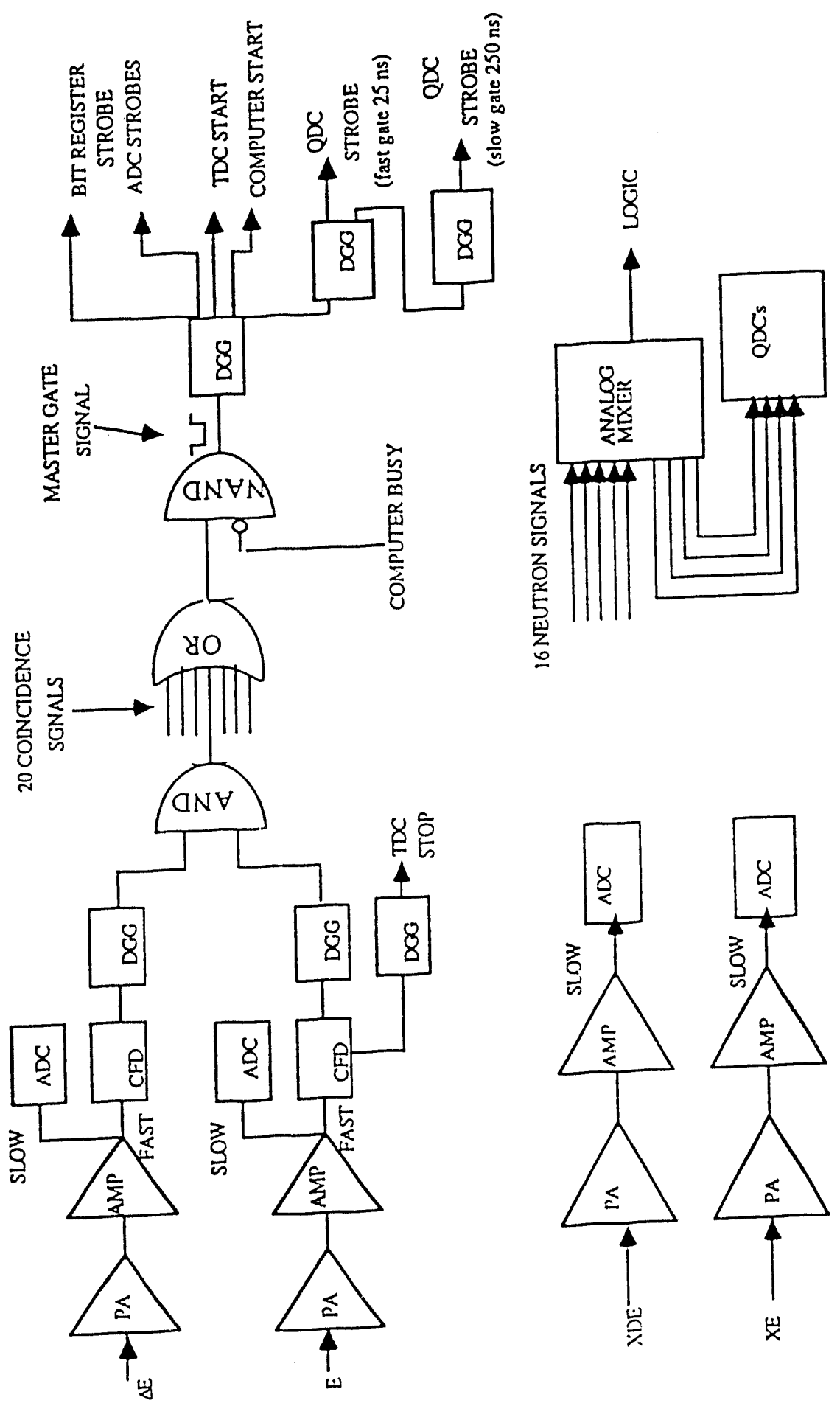

我 

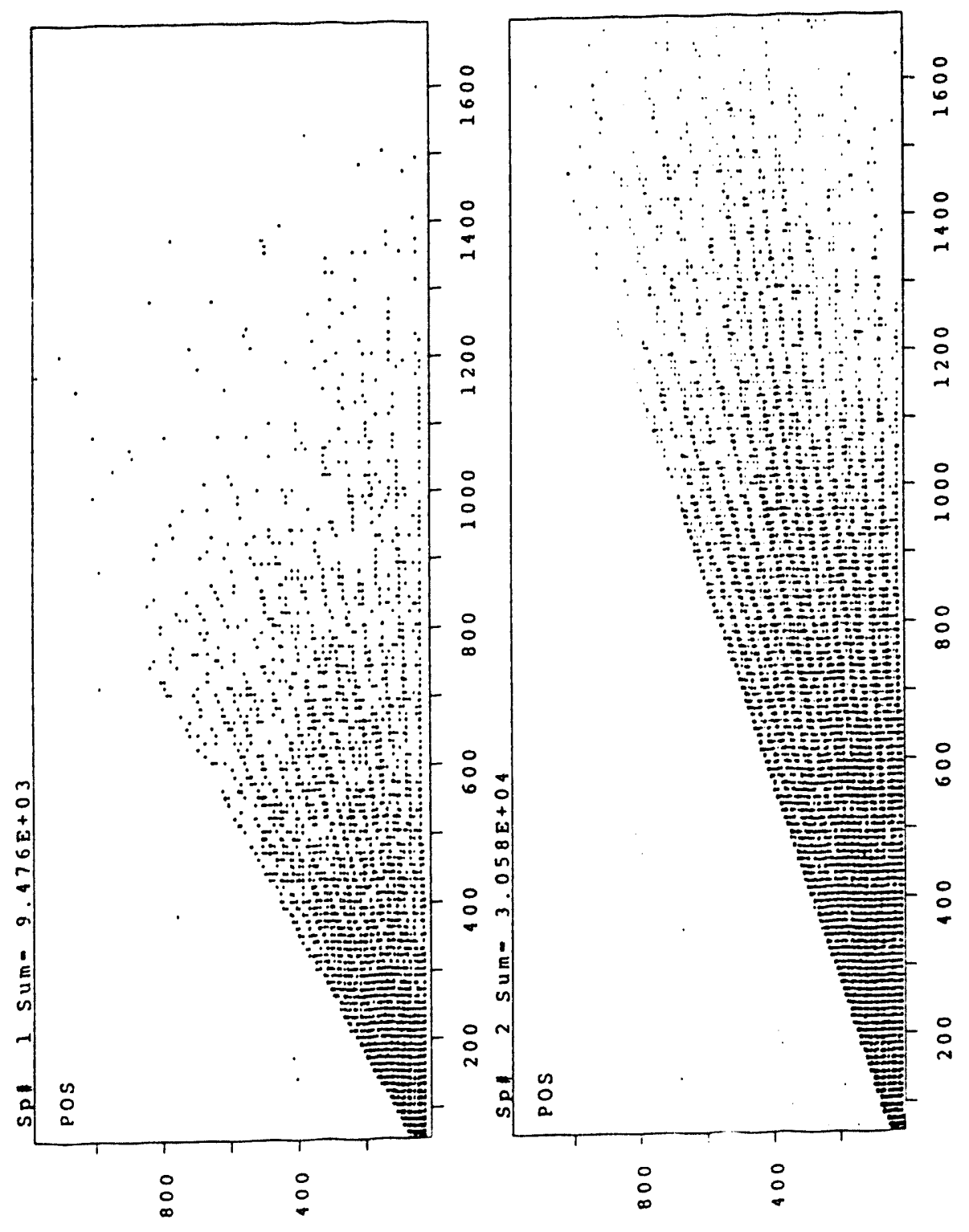

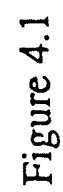




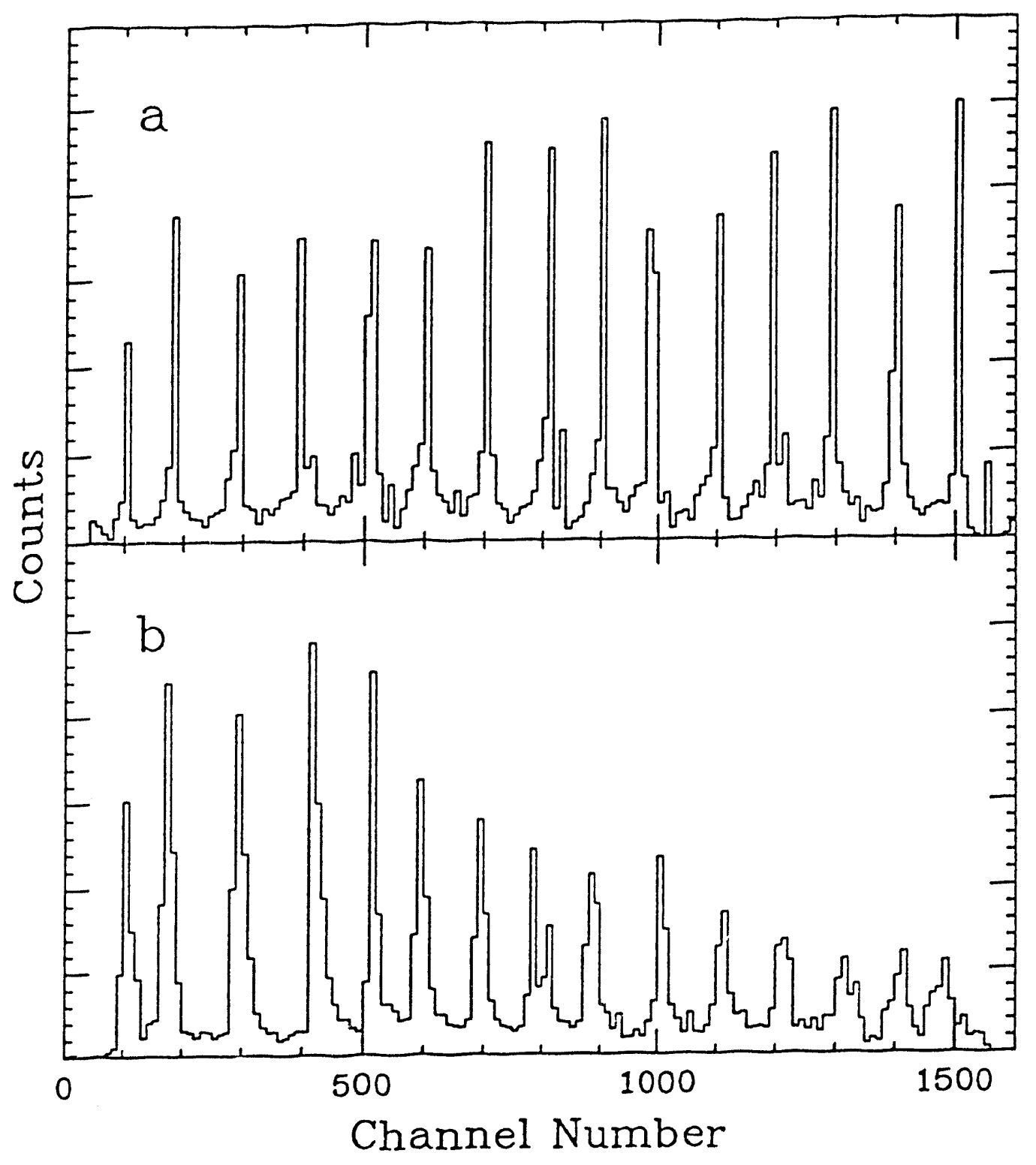

Figure 4.2 


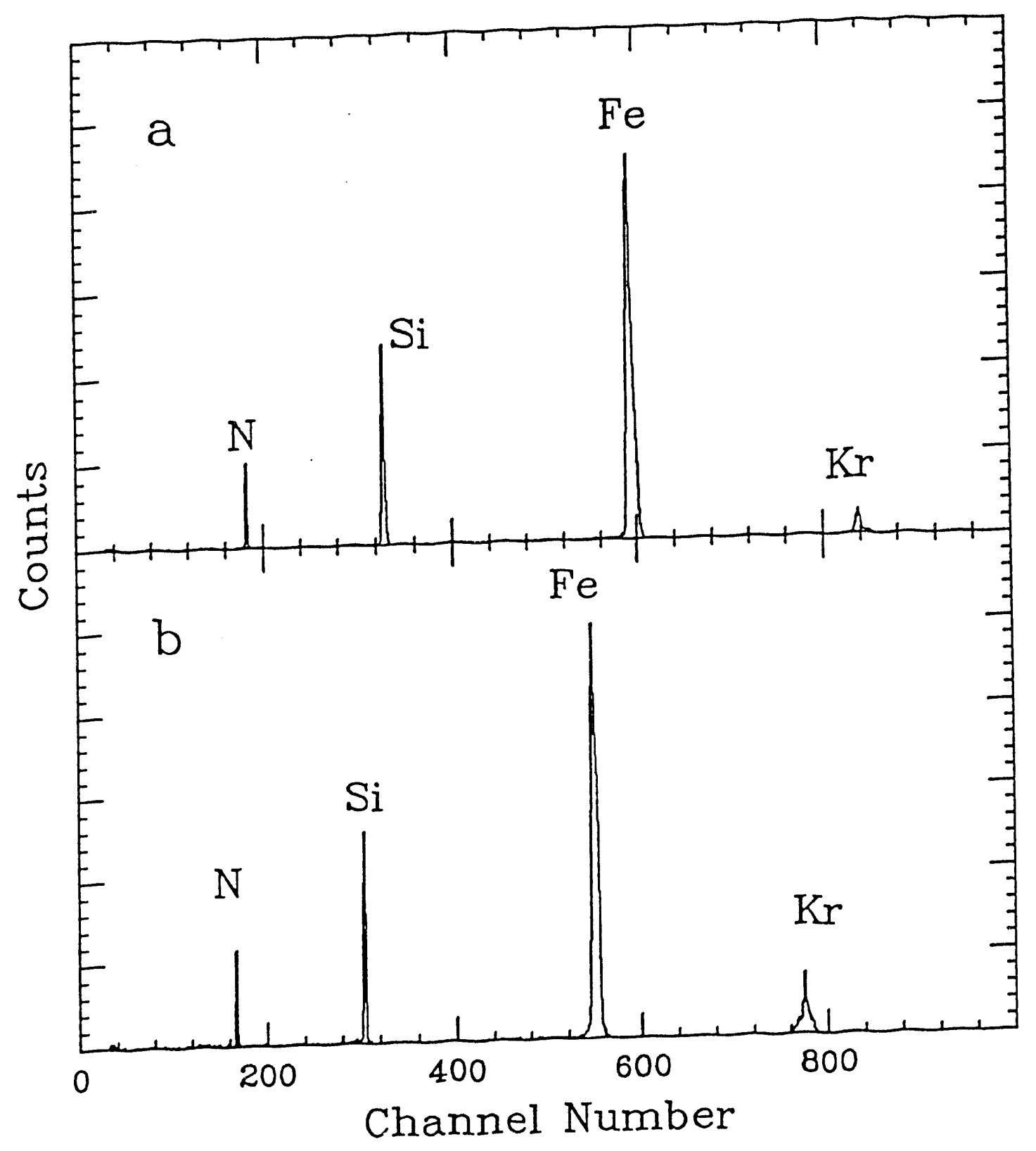

Figure 4.3 


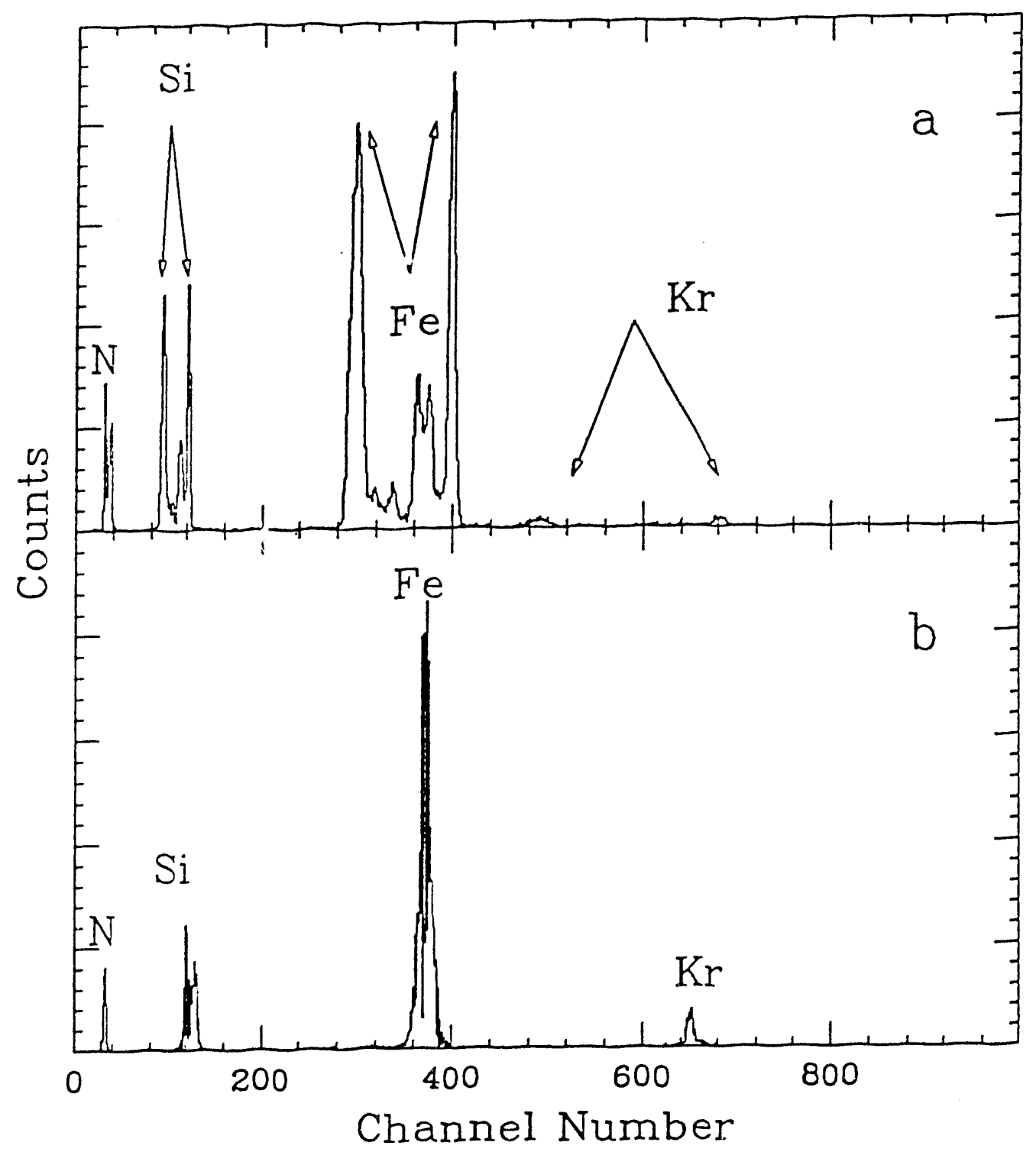

Figure 4.4 


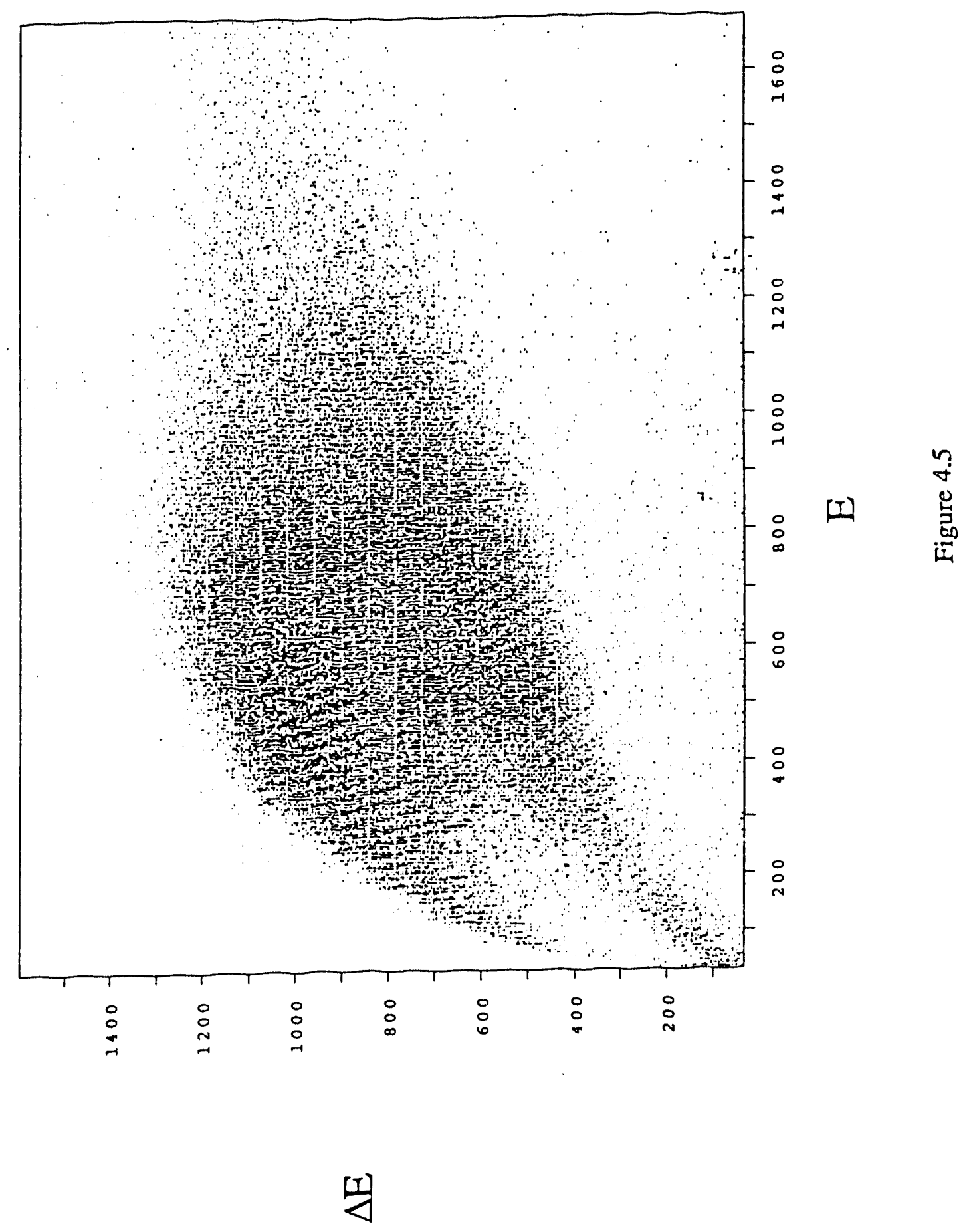




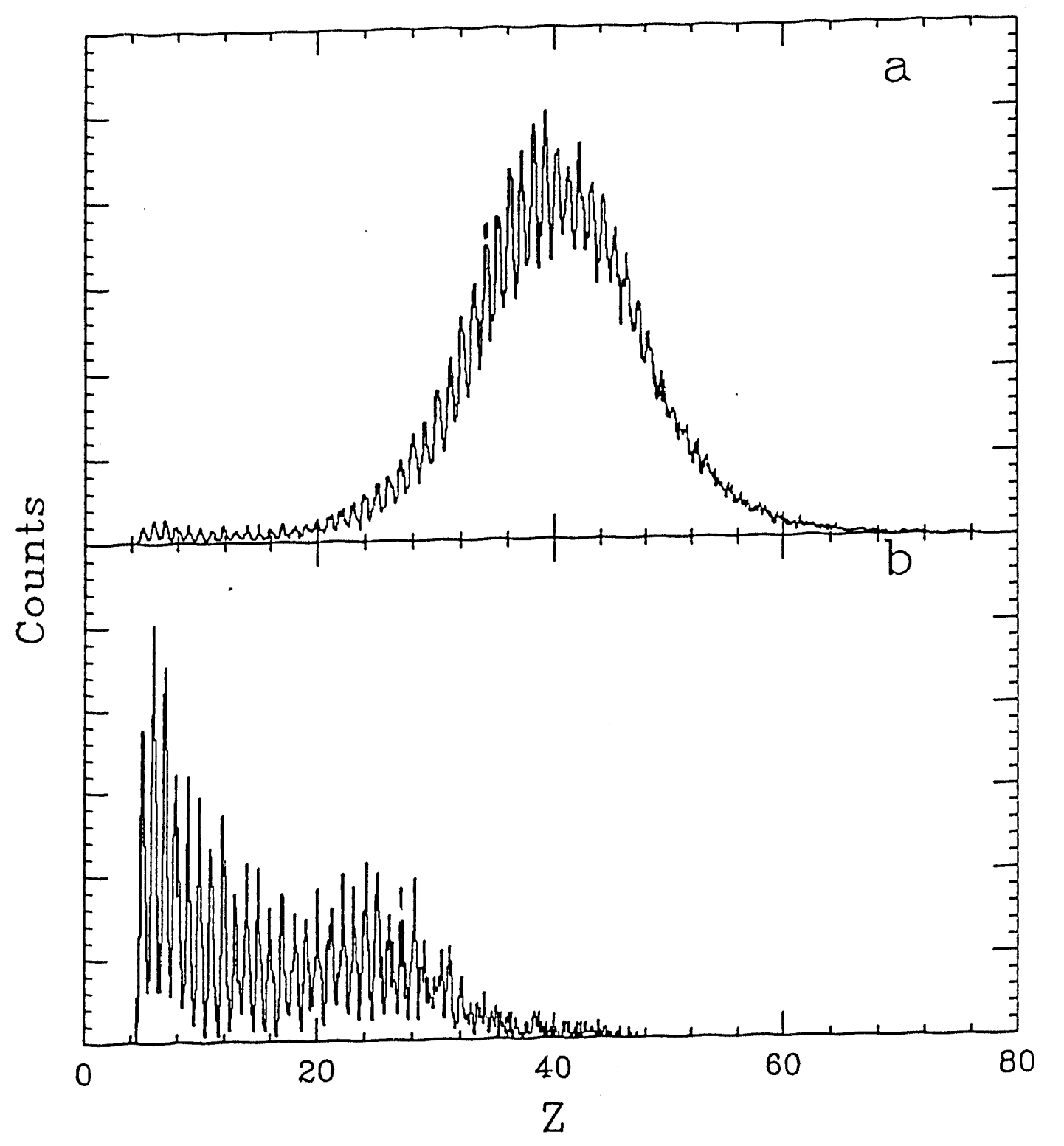

Figure 4.6 


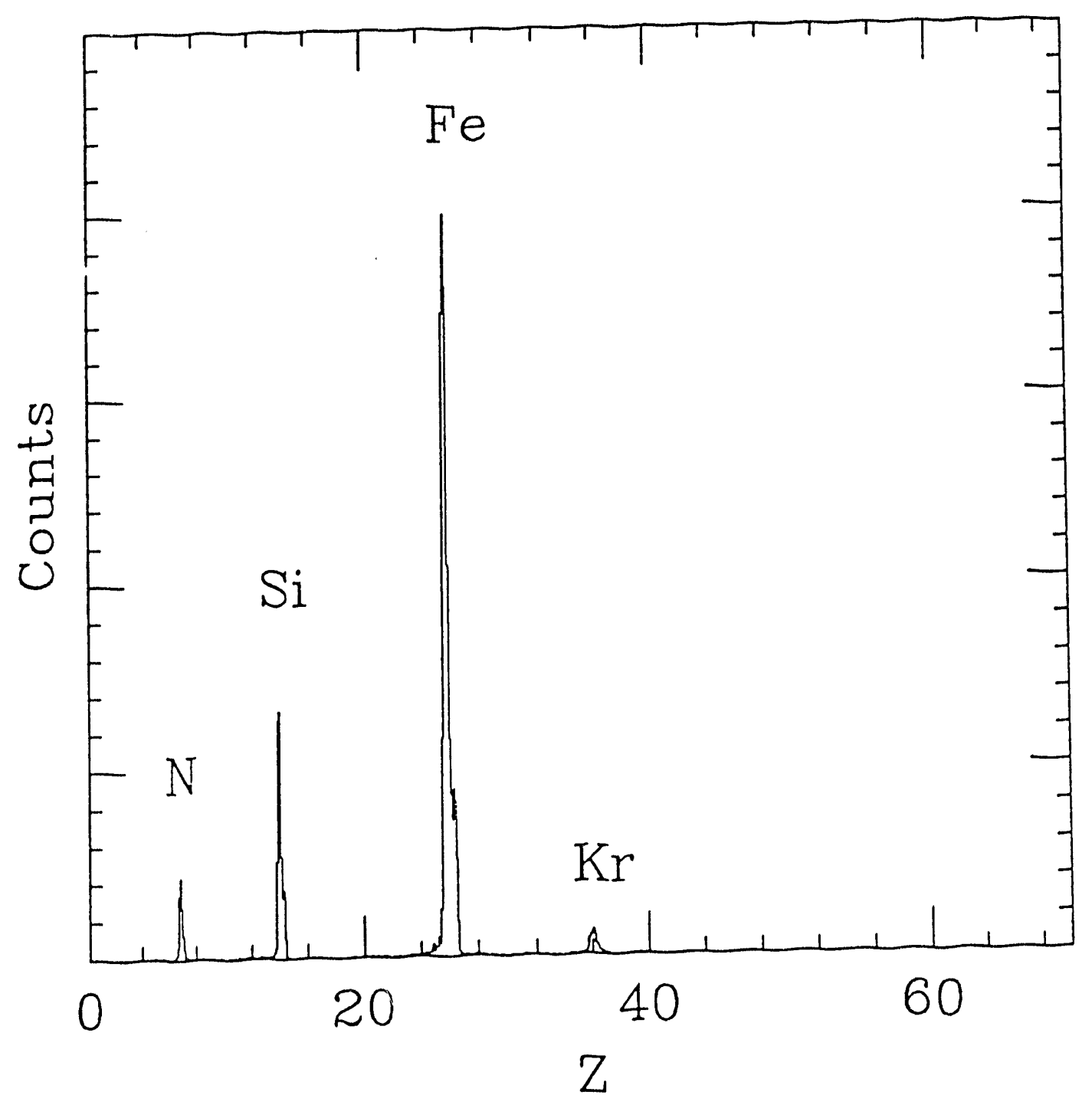

Figure 4.7 


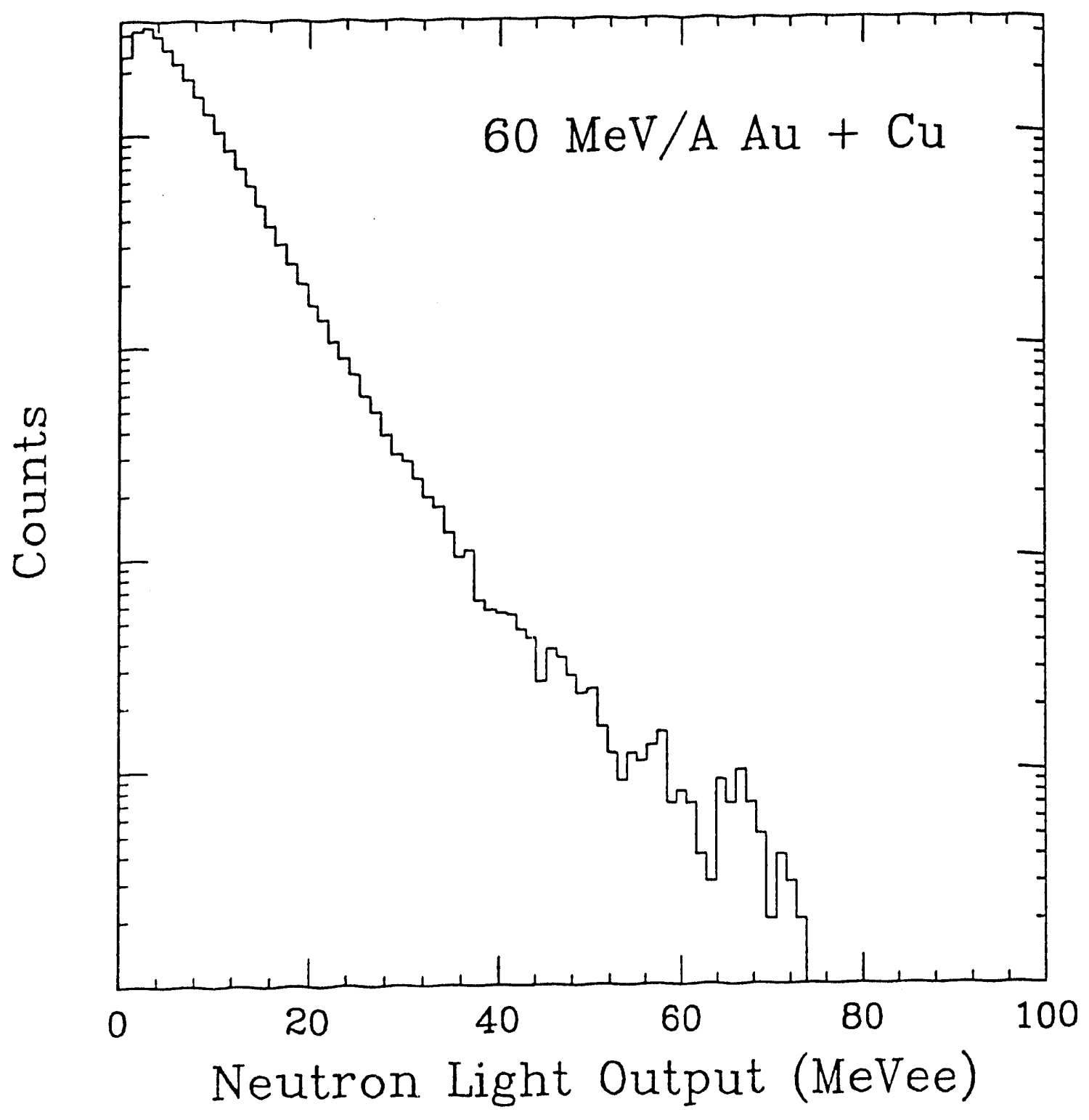

Figure 4.8 


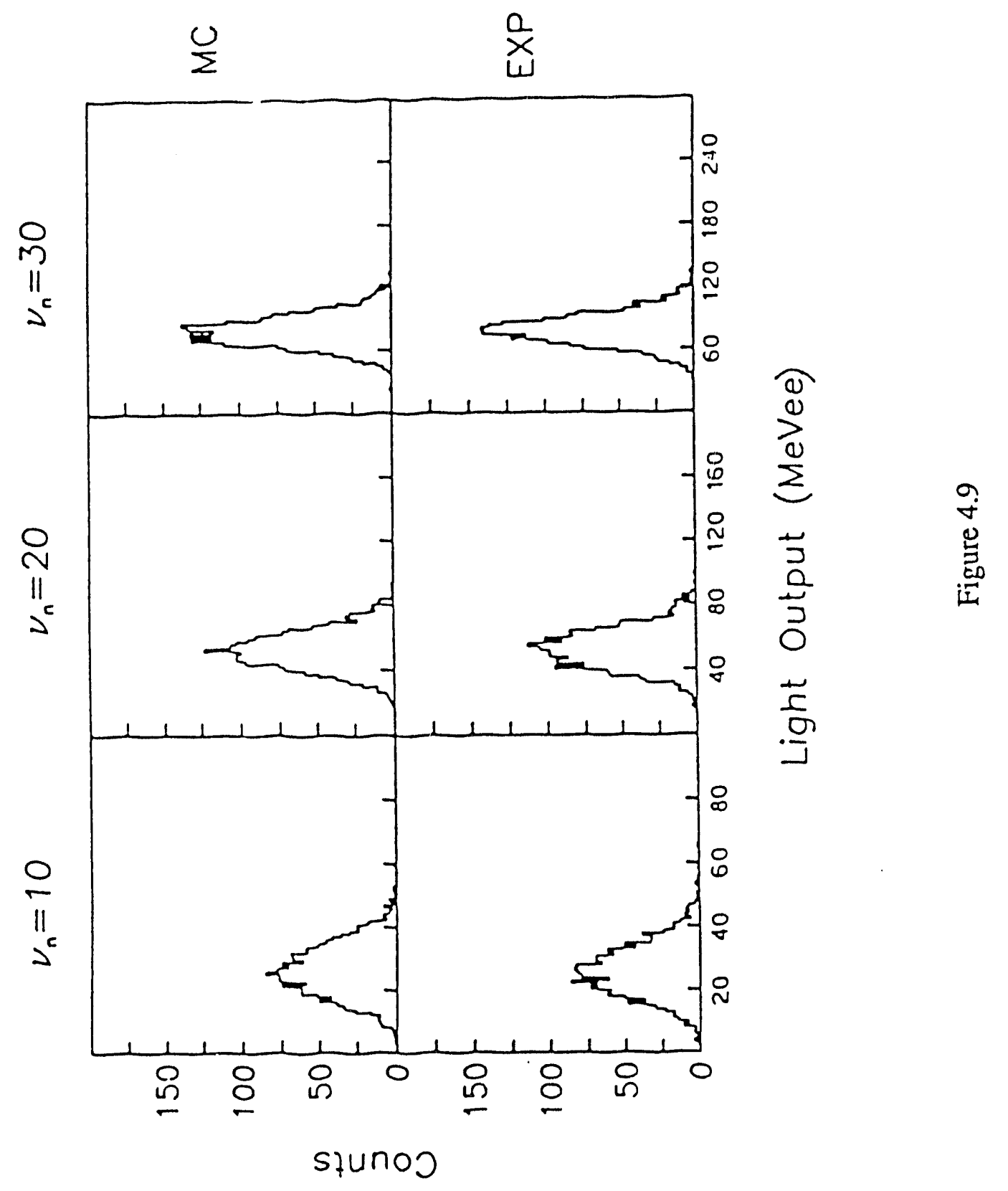




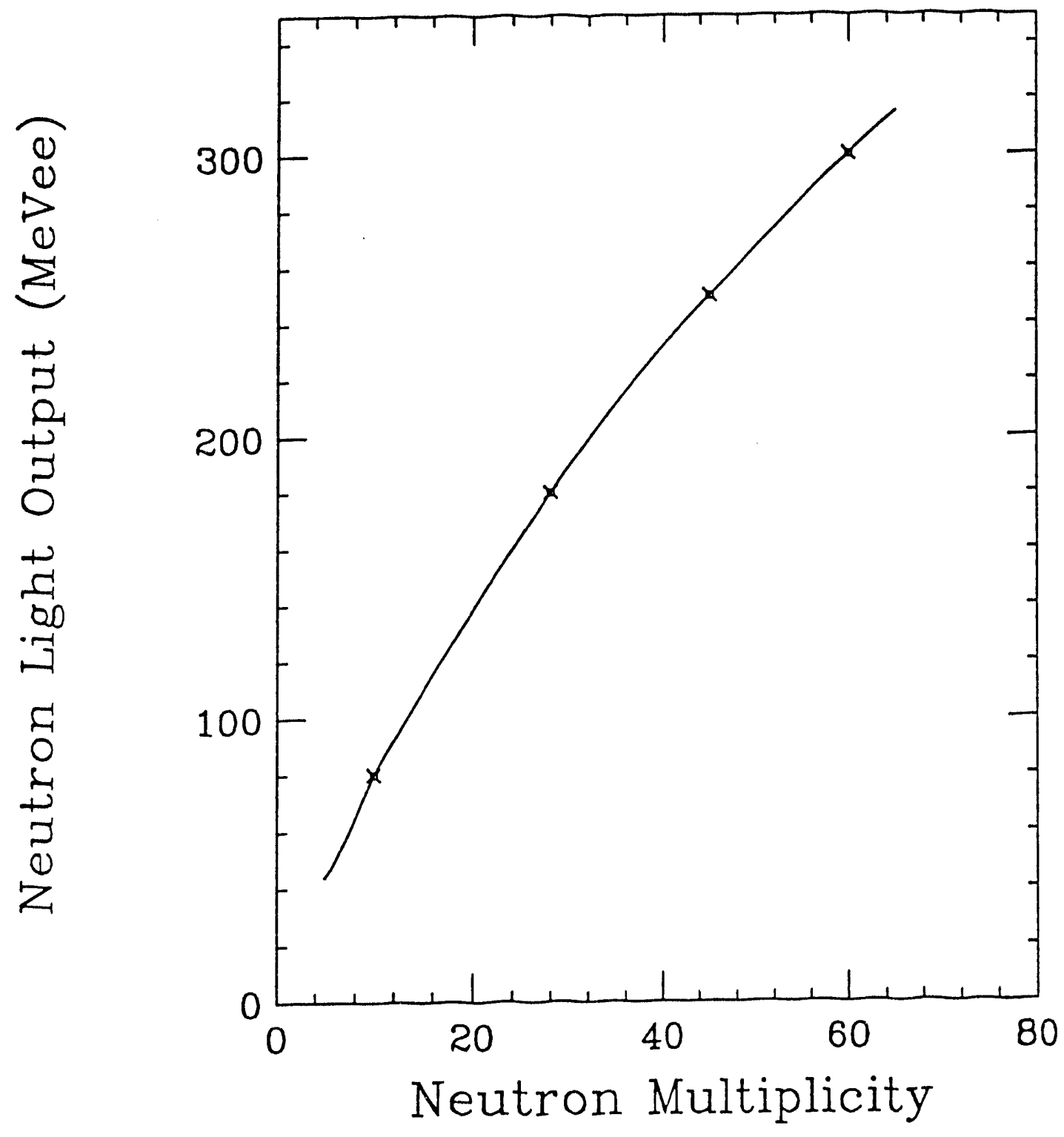

Figure 4.10 


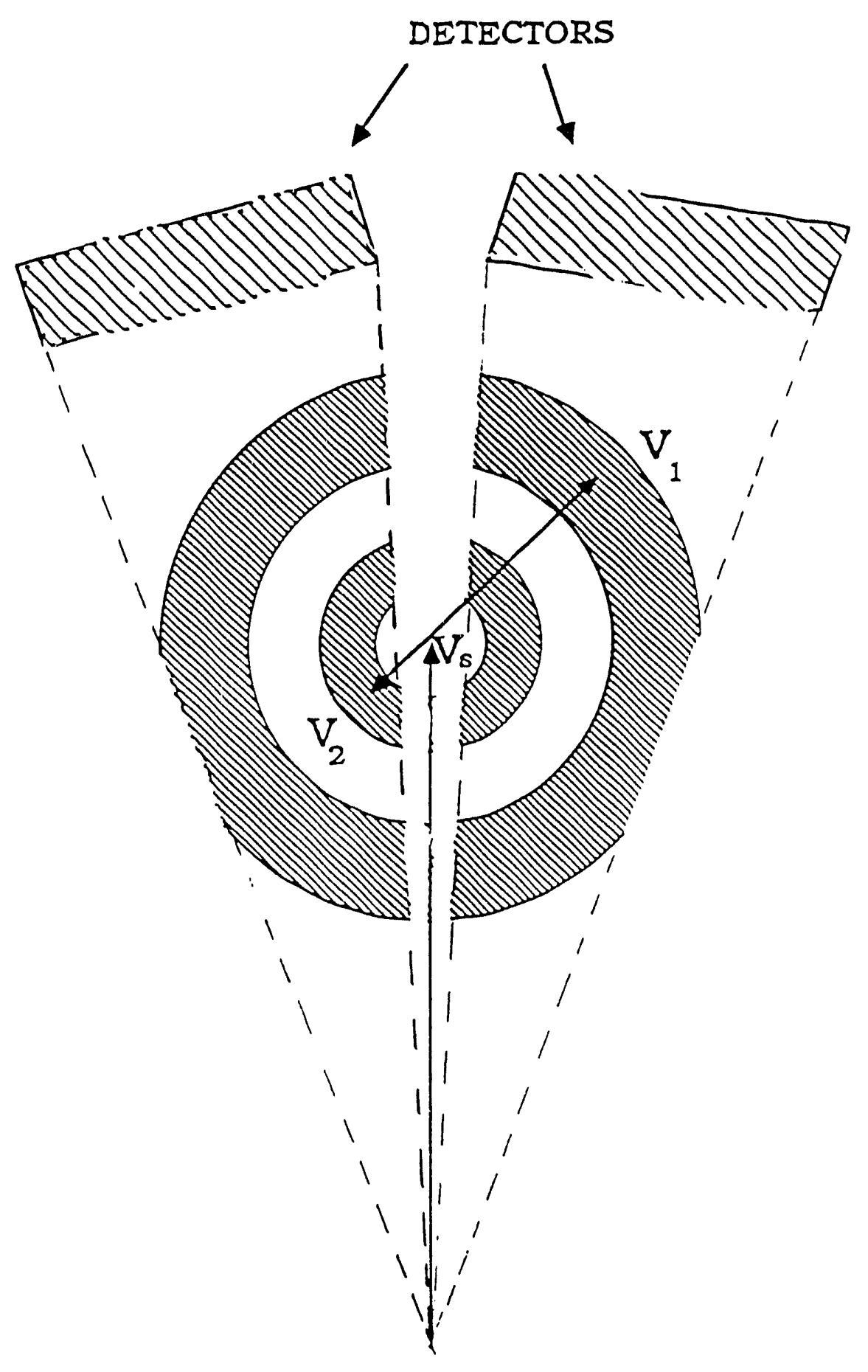

Figure 5.1 


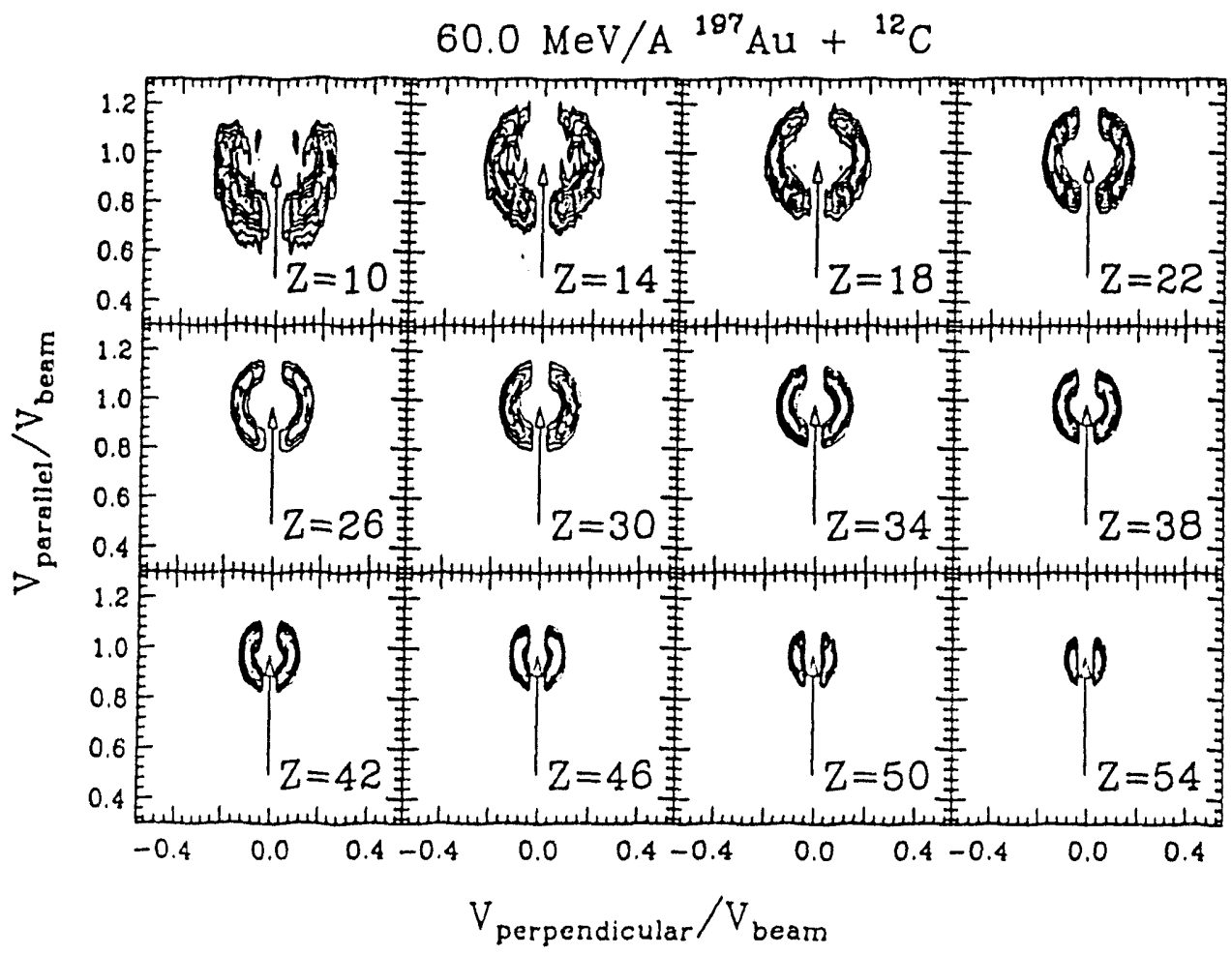

Figure 5.2a

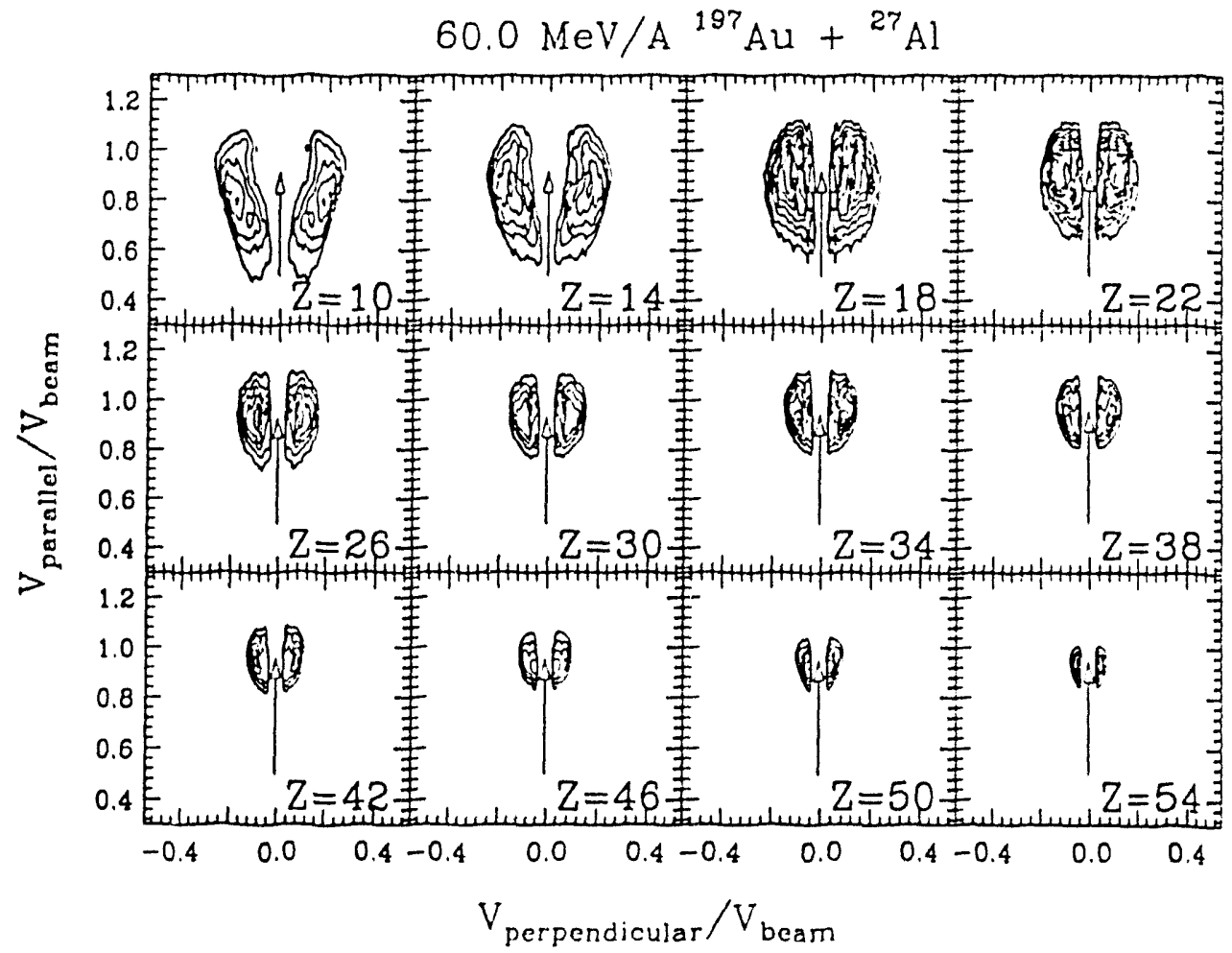

Figure $5.2 b$ 


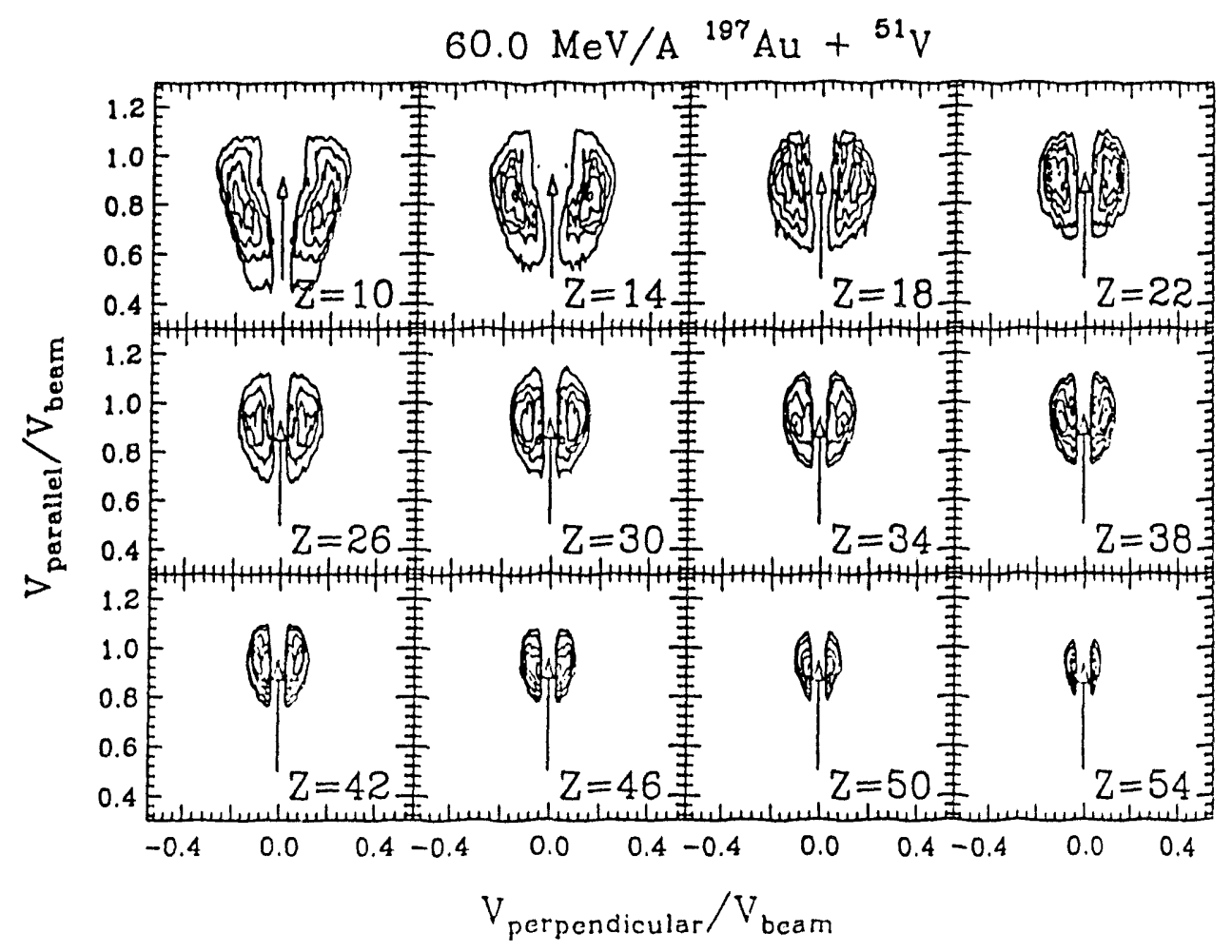

Figure 5.2c

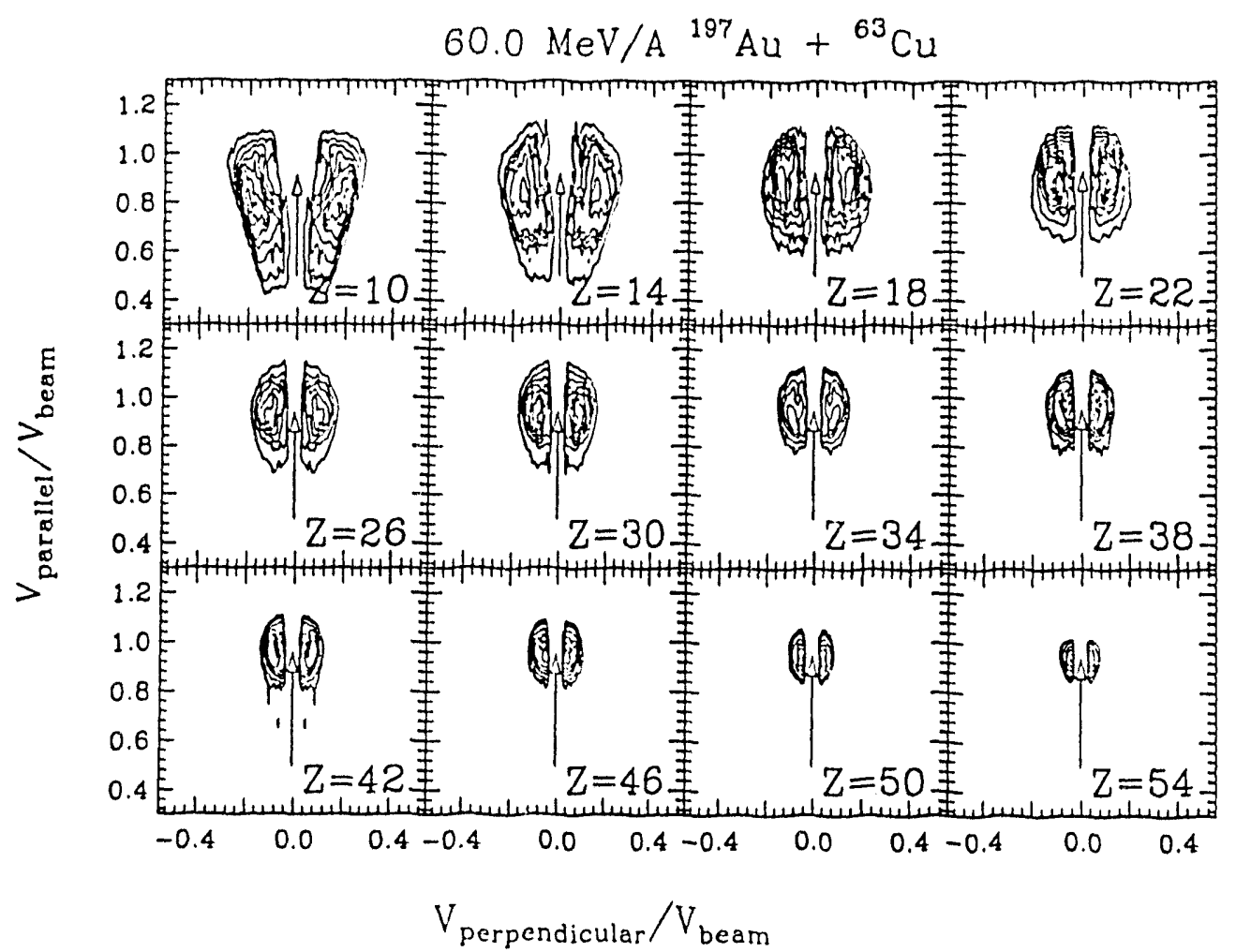

Figure 5.2d 


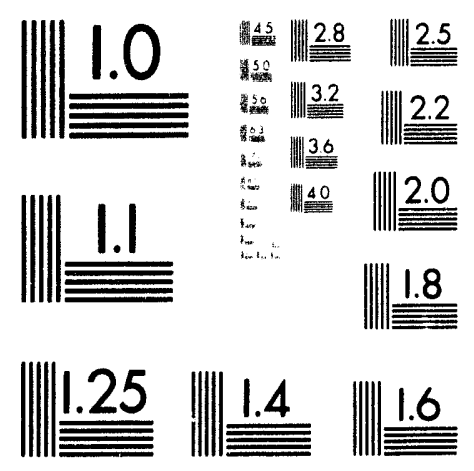



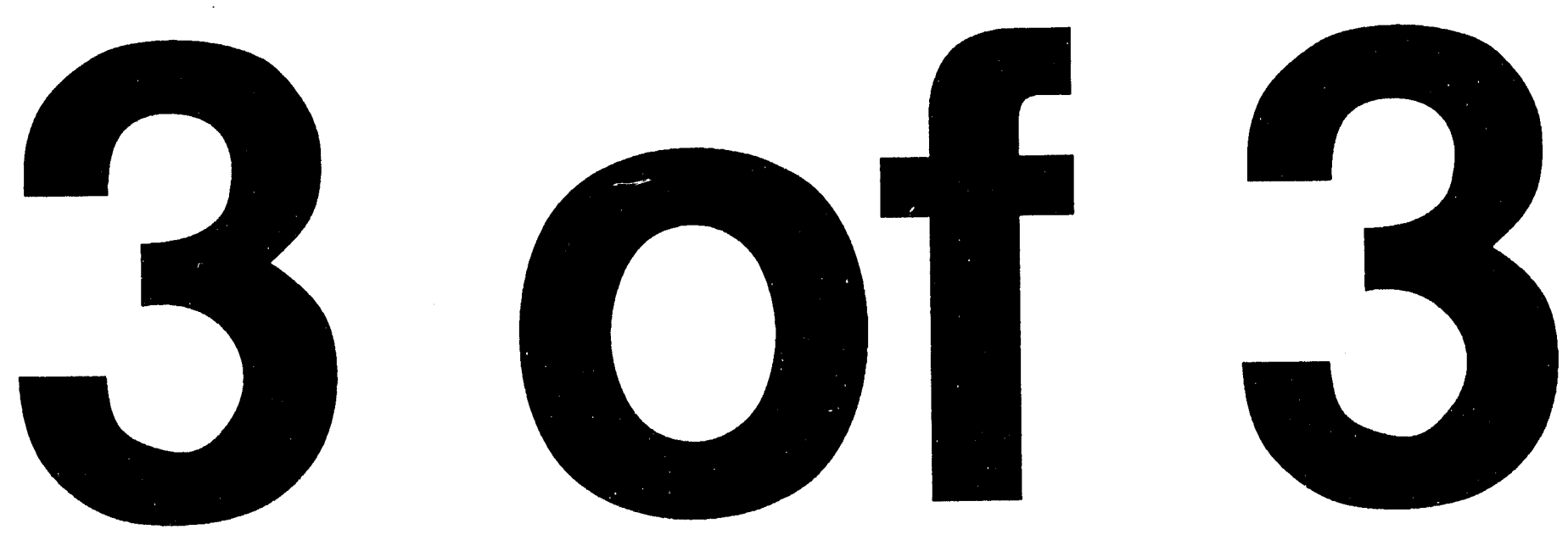


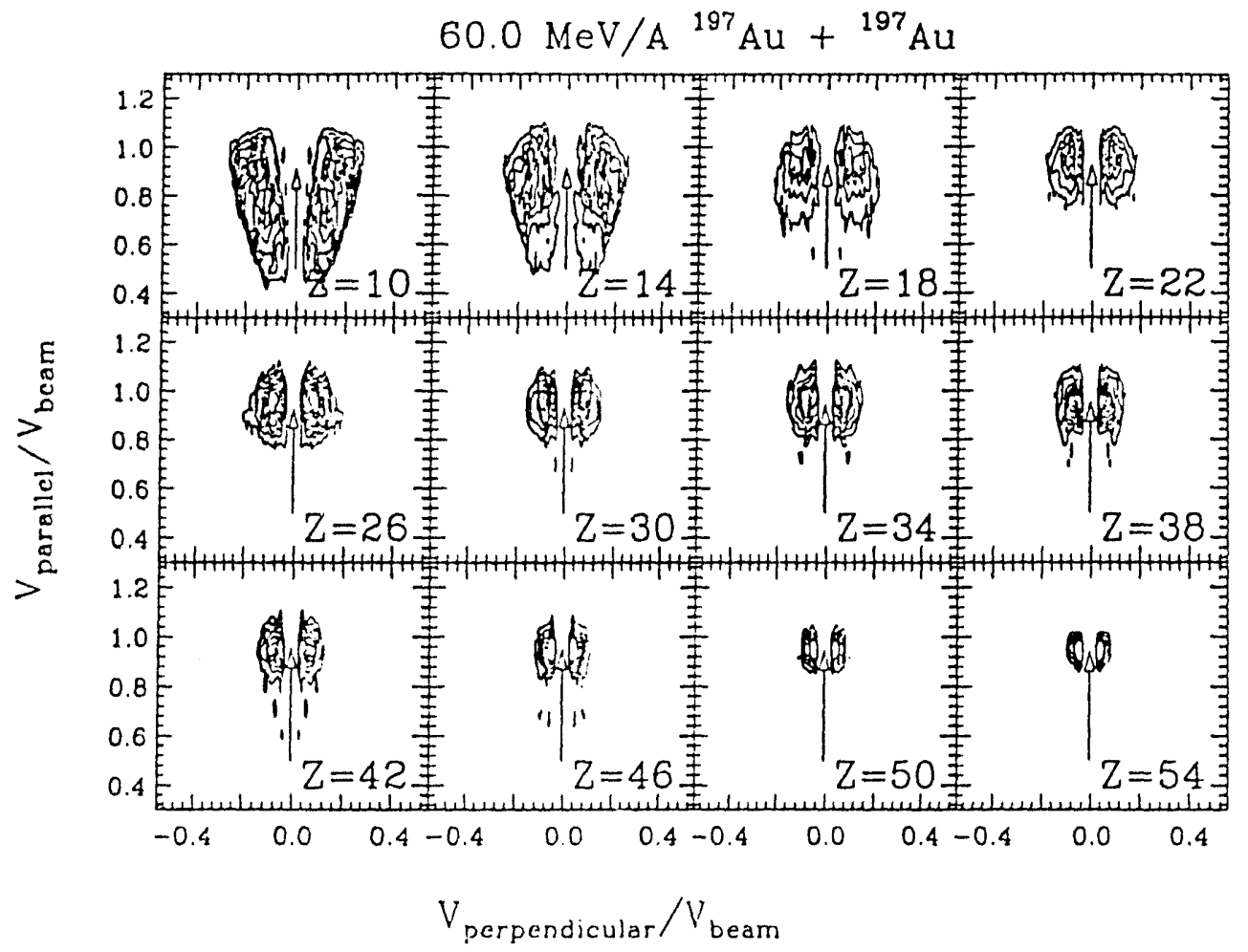

Figure $5.2 \mathrm{e}$

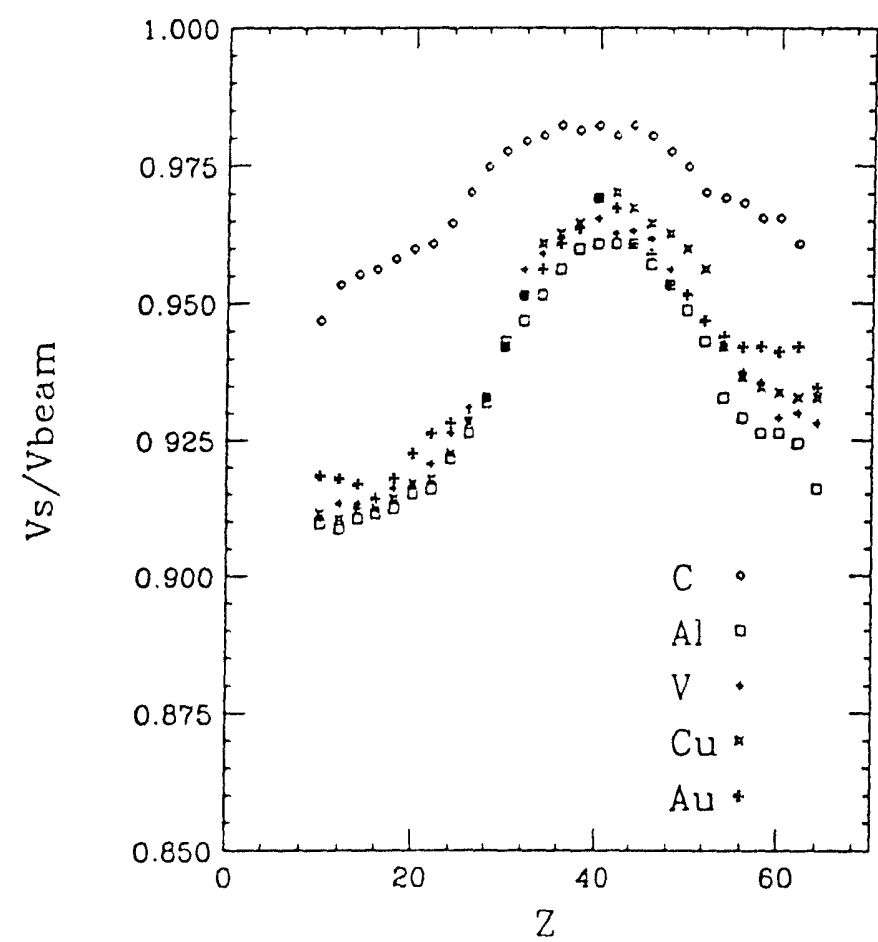

Figure 5.3 


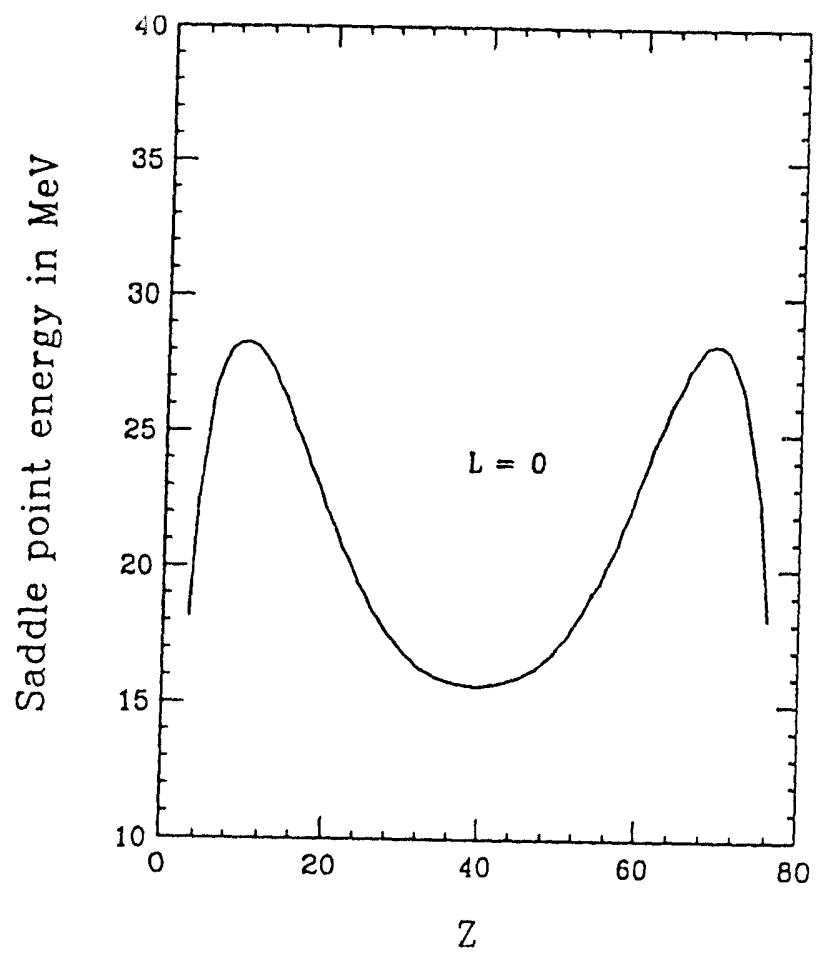

Figure 5.4

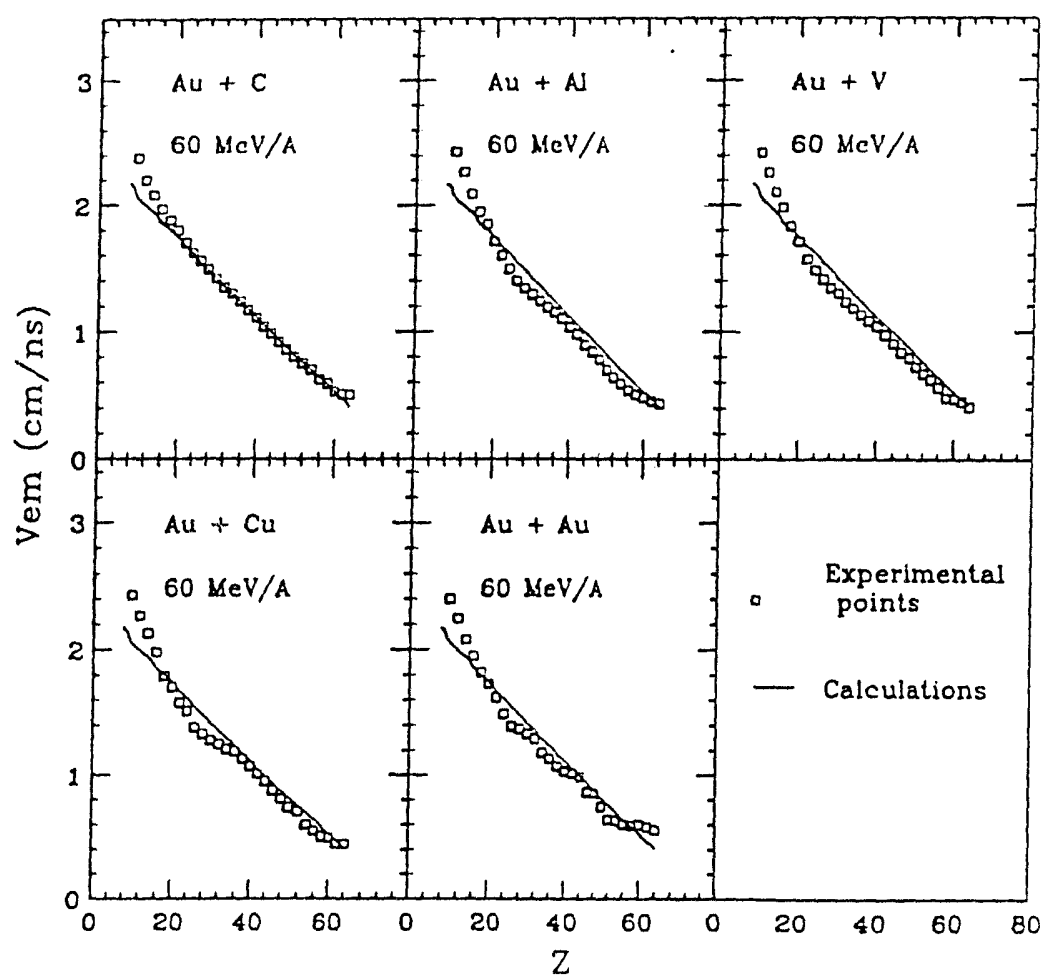

Figure 5.5 


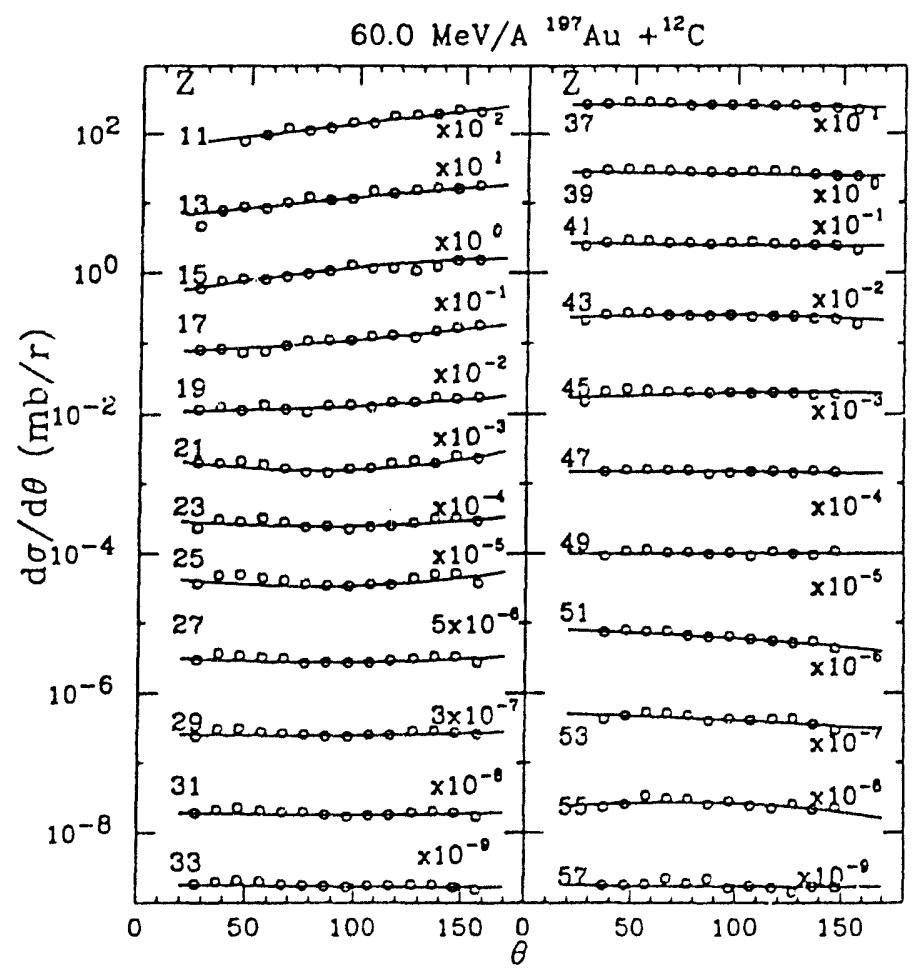

Figure $5.6 \mathrm{a}$

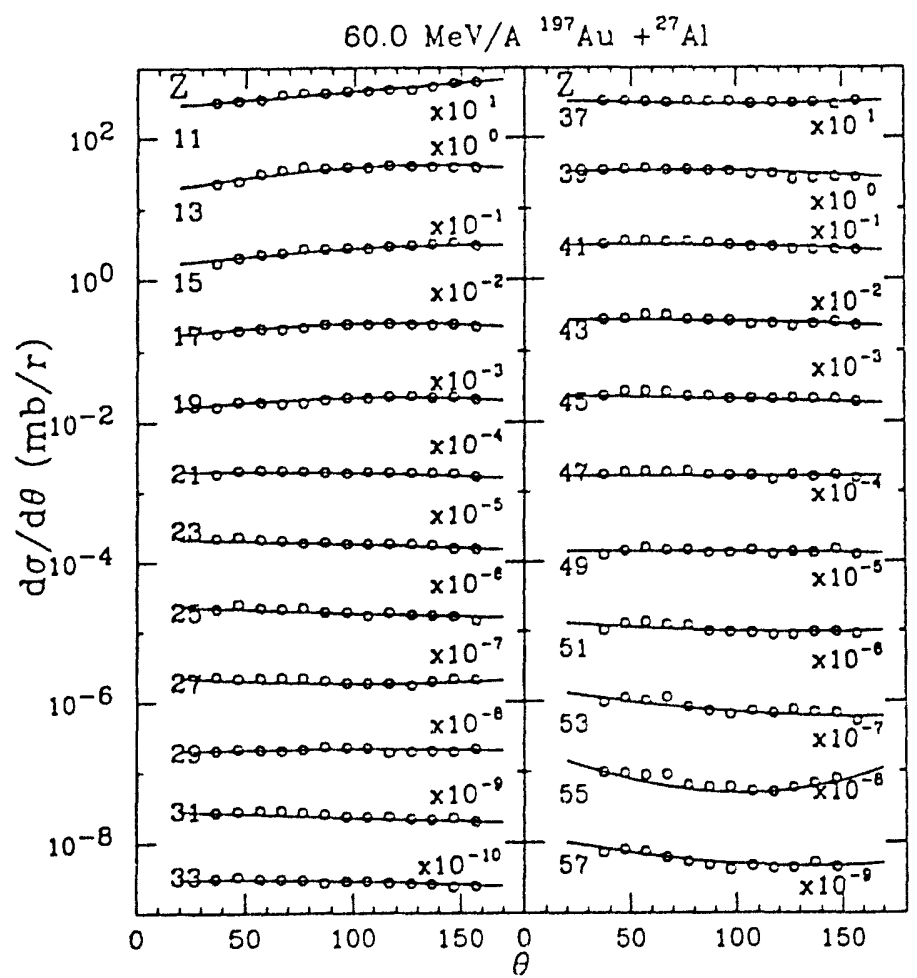

Figure $5.6 \mathrm{~b}$ 


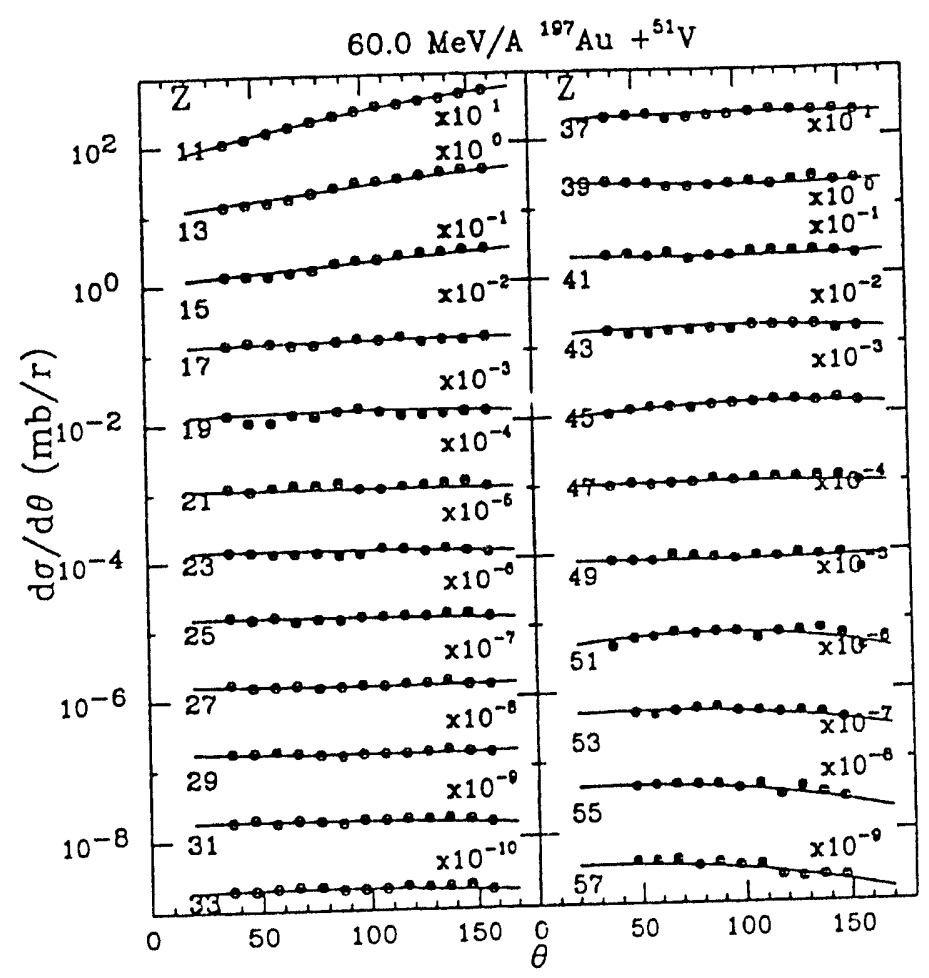

Figure 5.6c

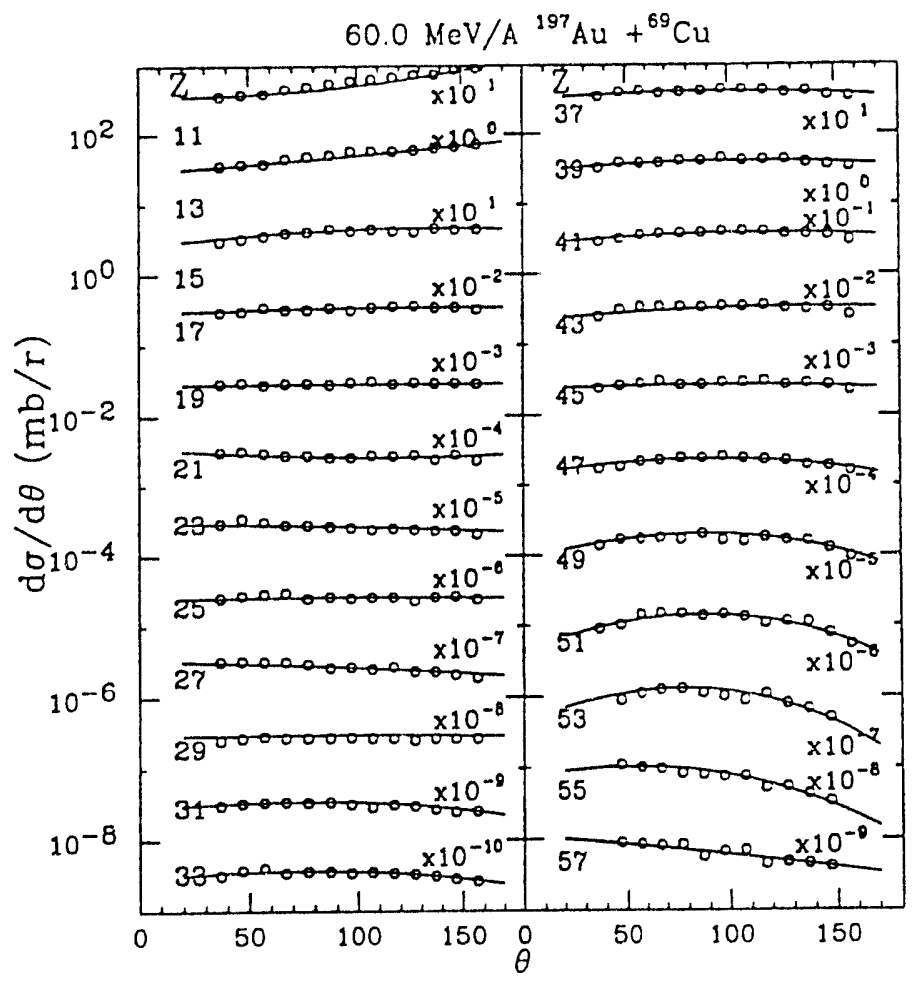

Figure 5.6d 


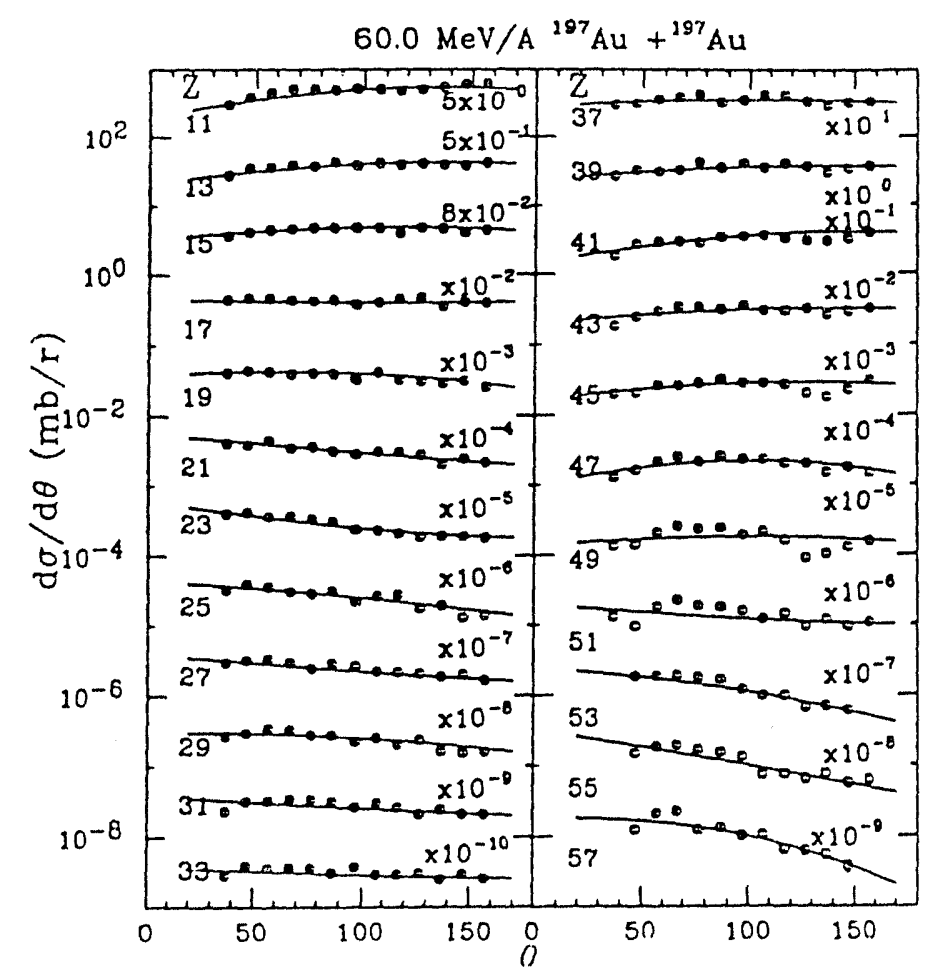

Figure 5.6e

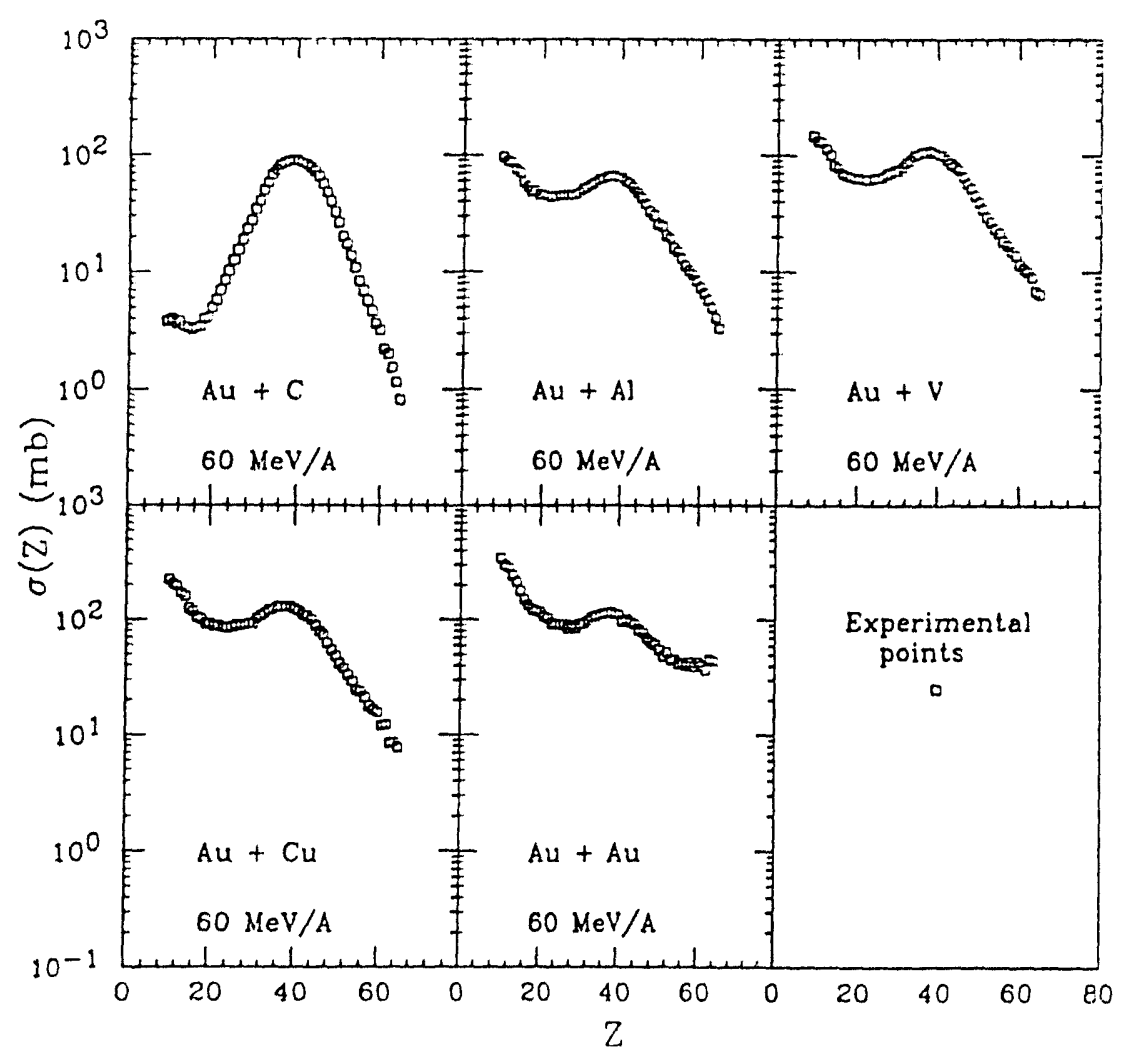

Figure 5.7 


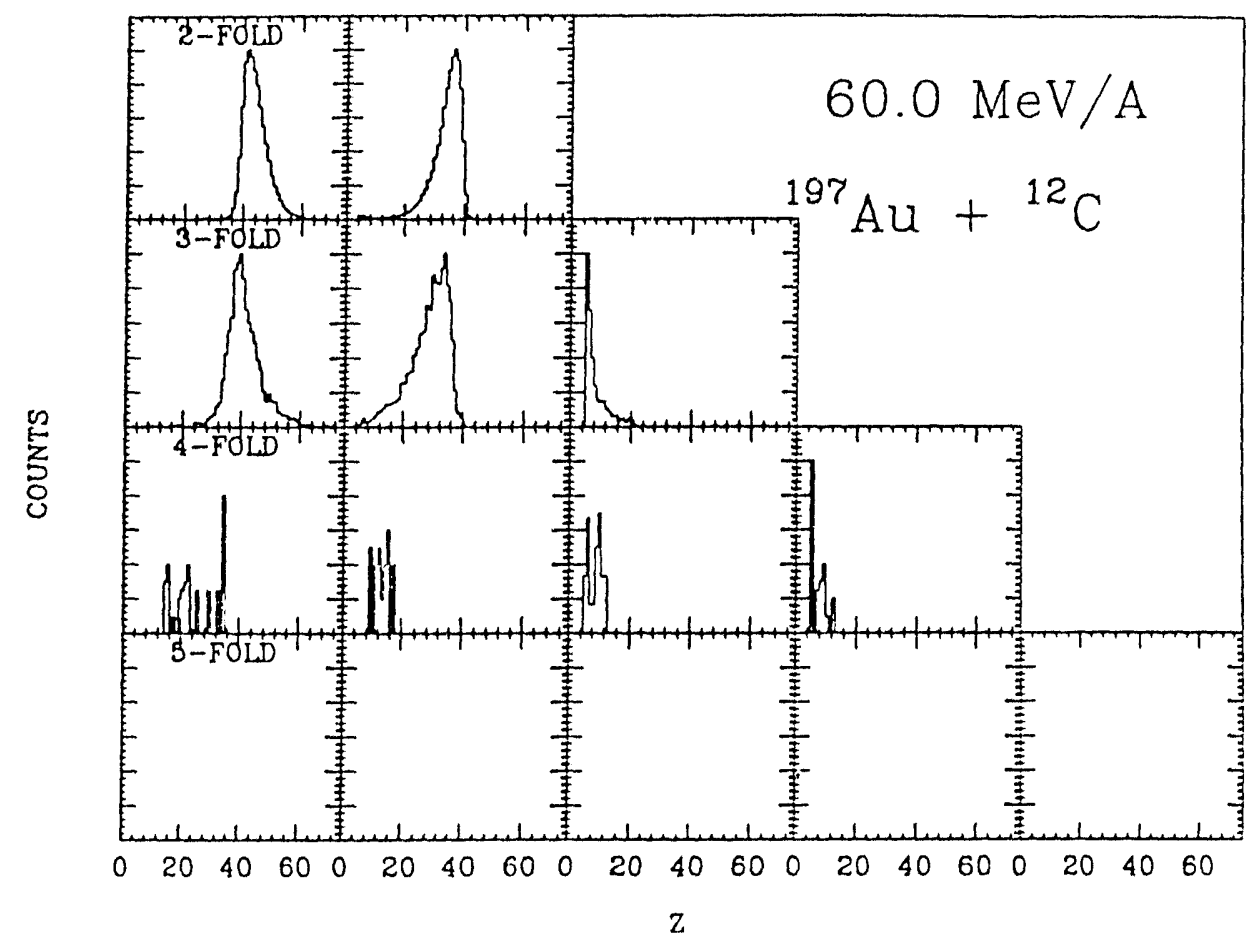

Figure 5.8a

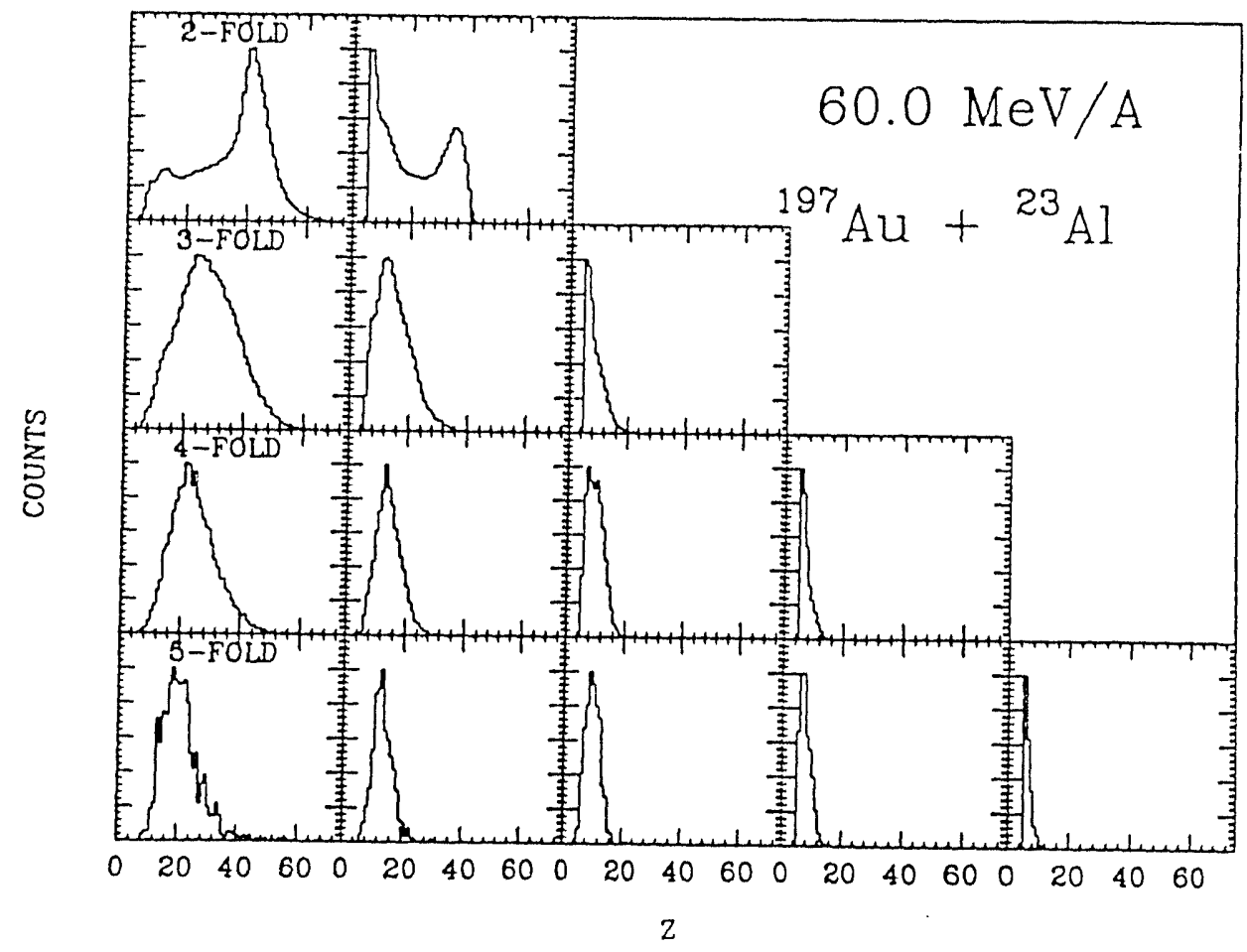

Figure $5.8 b$ 


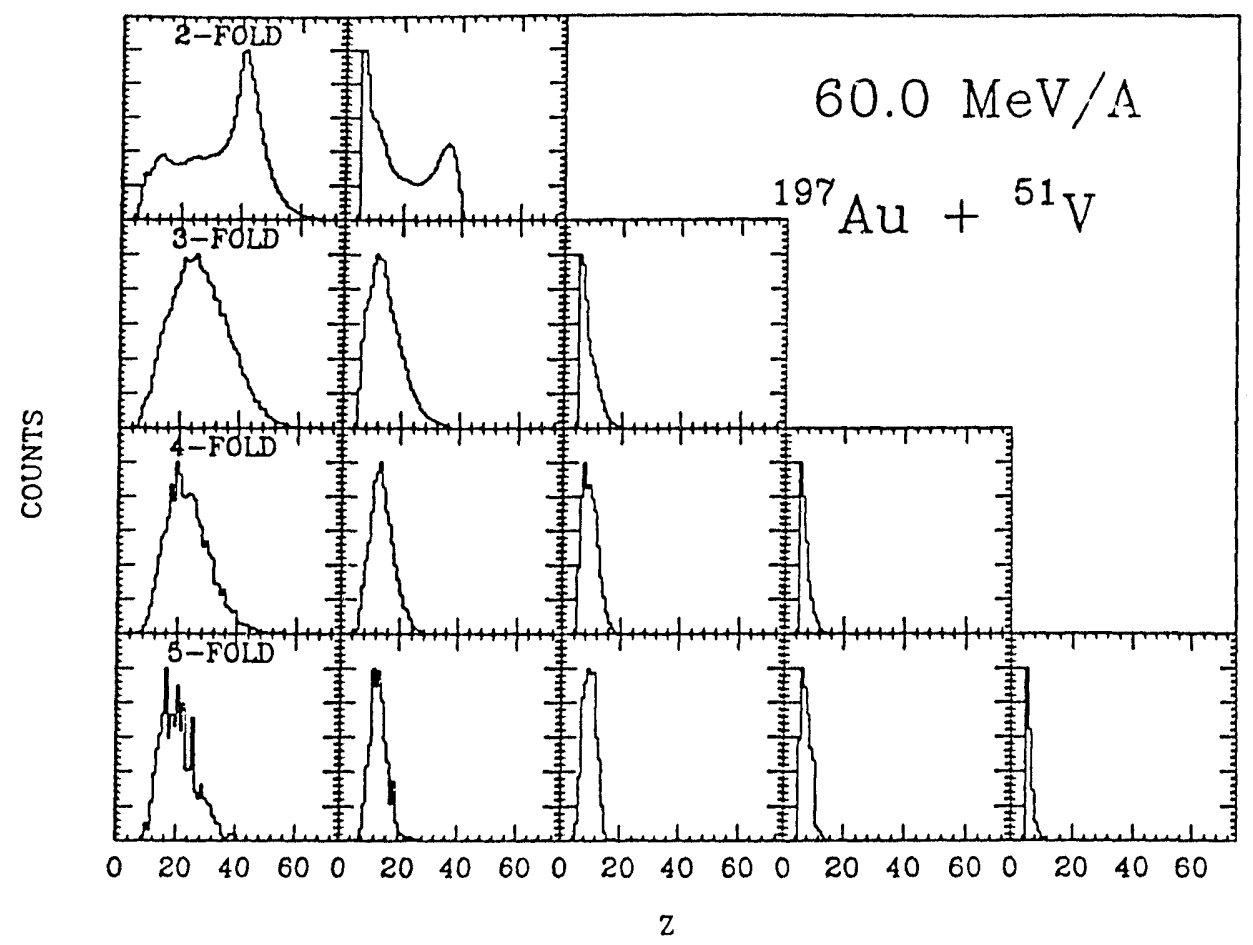

Figure 5.8c

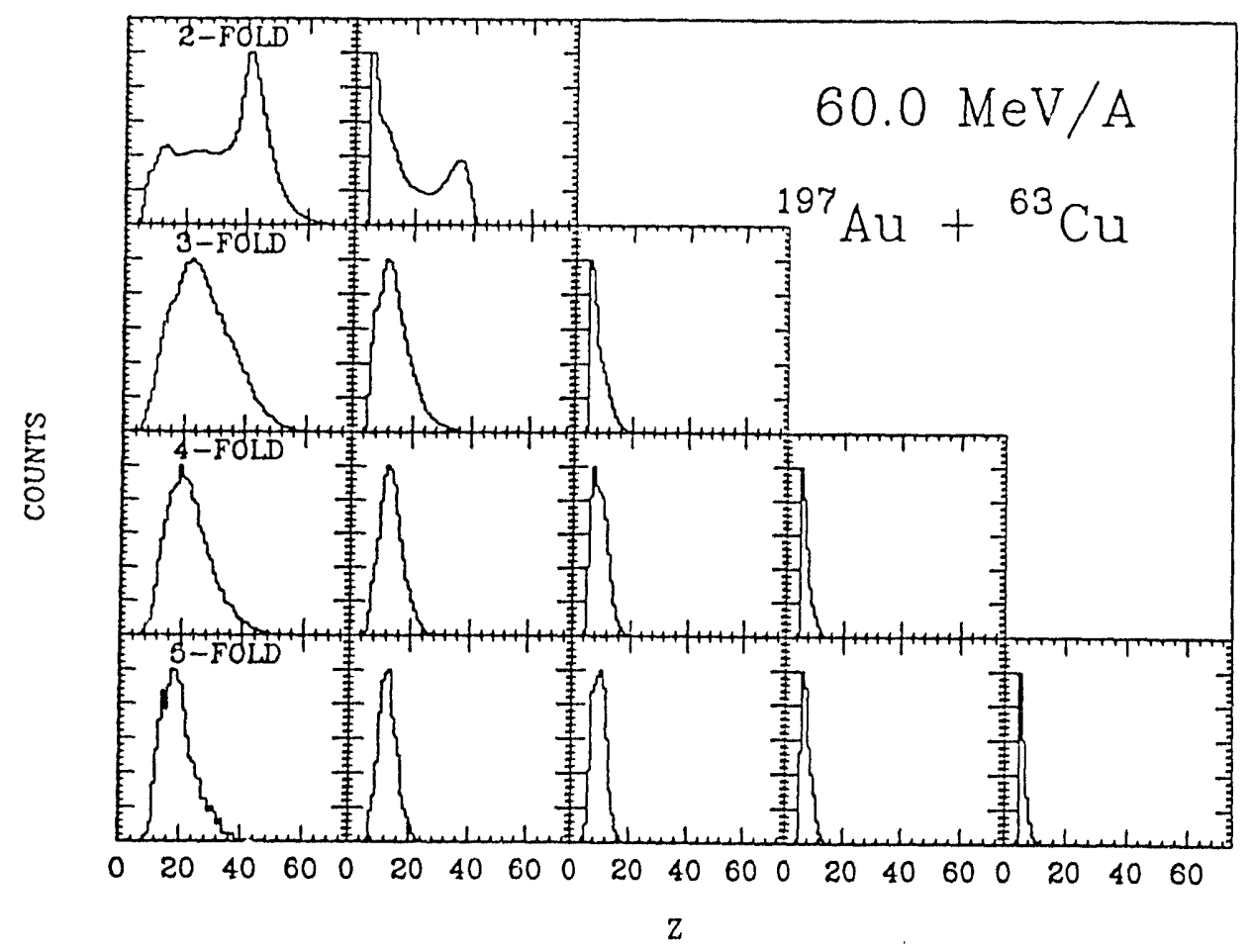

Figure 5.8d 


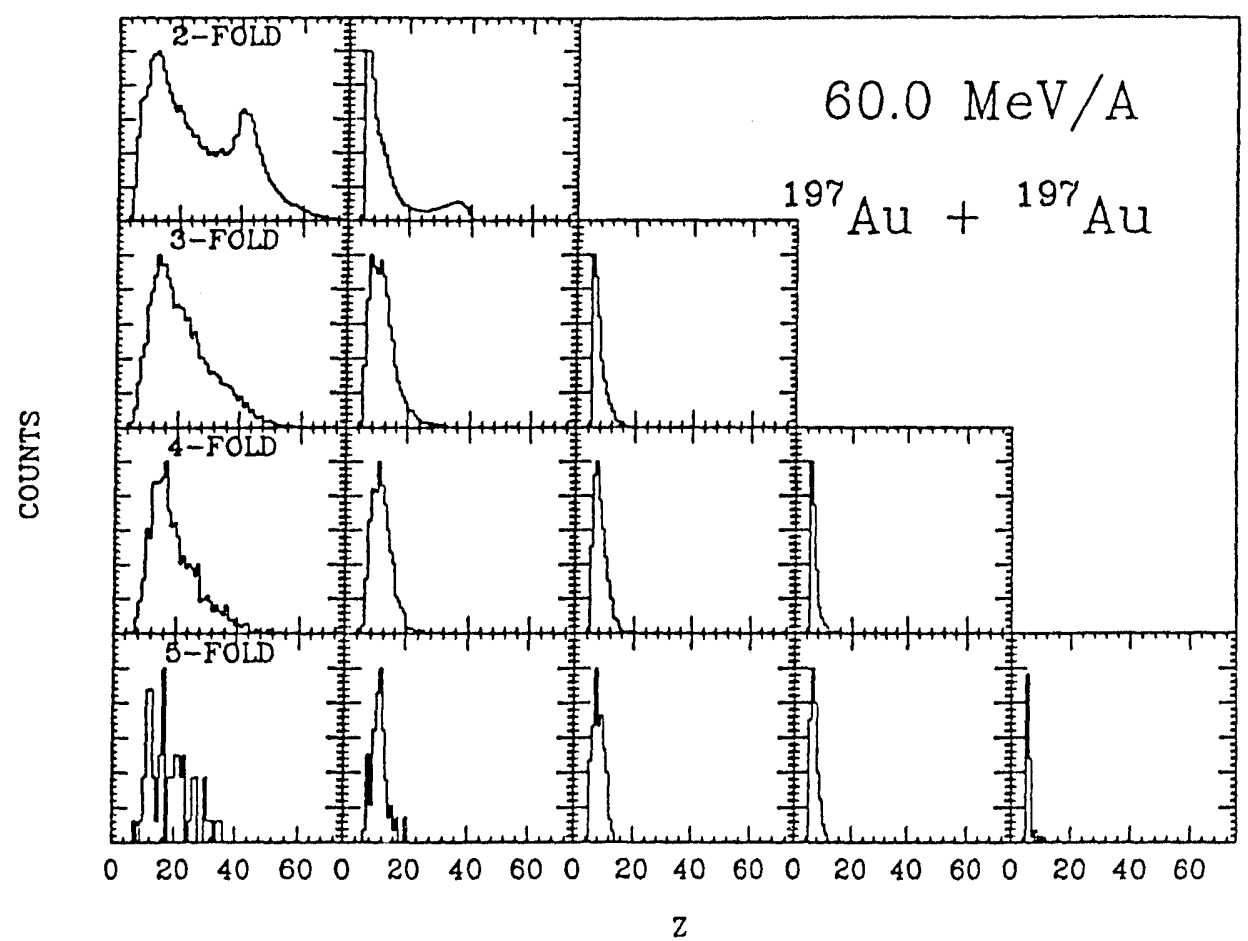

Figure $5.8 \mathrm{e}$ 


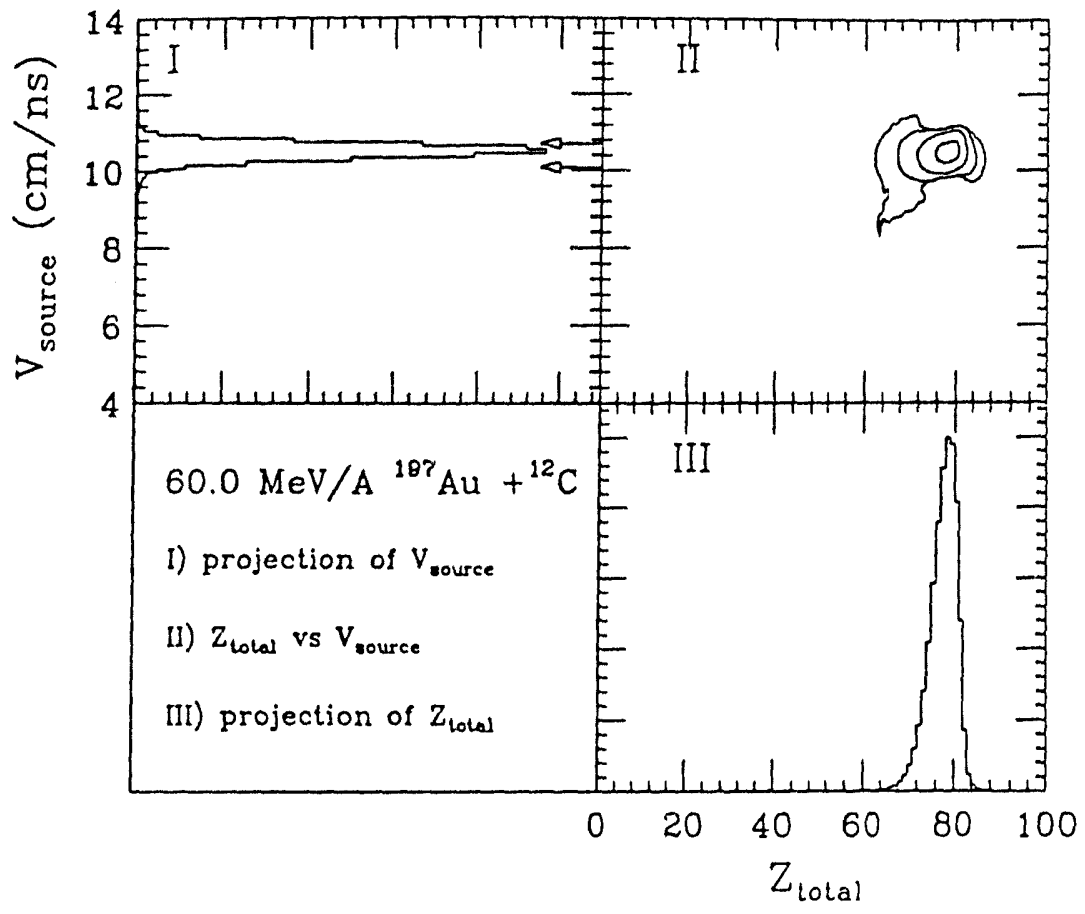

Figure 5.9a

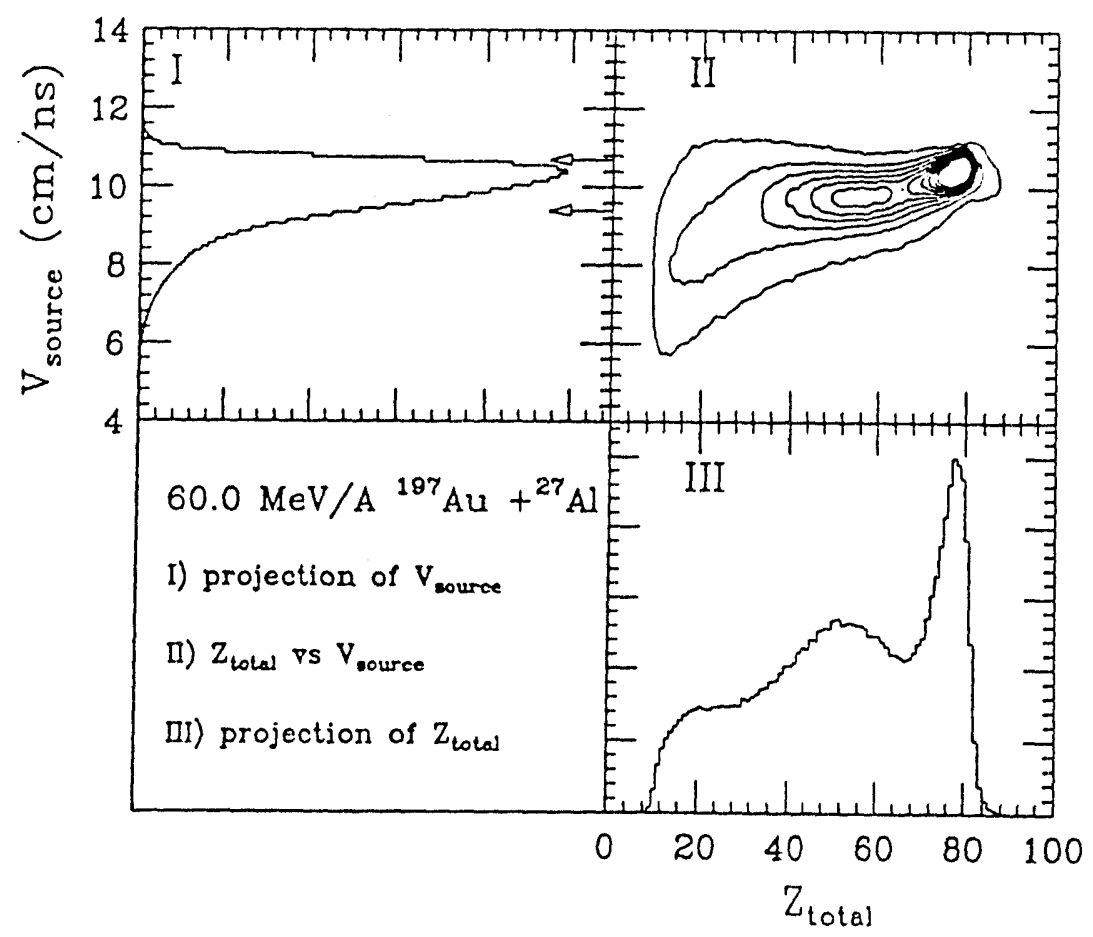

Figure 5.9b 


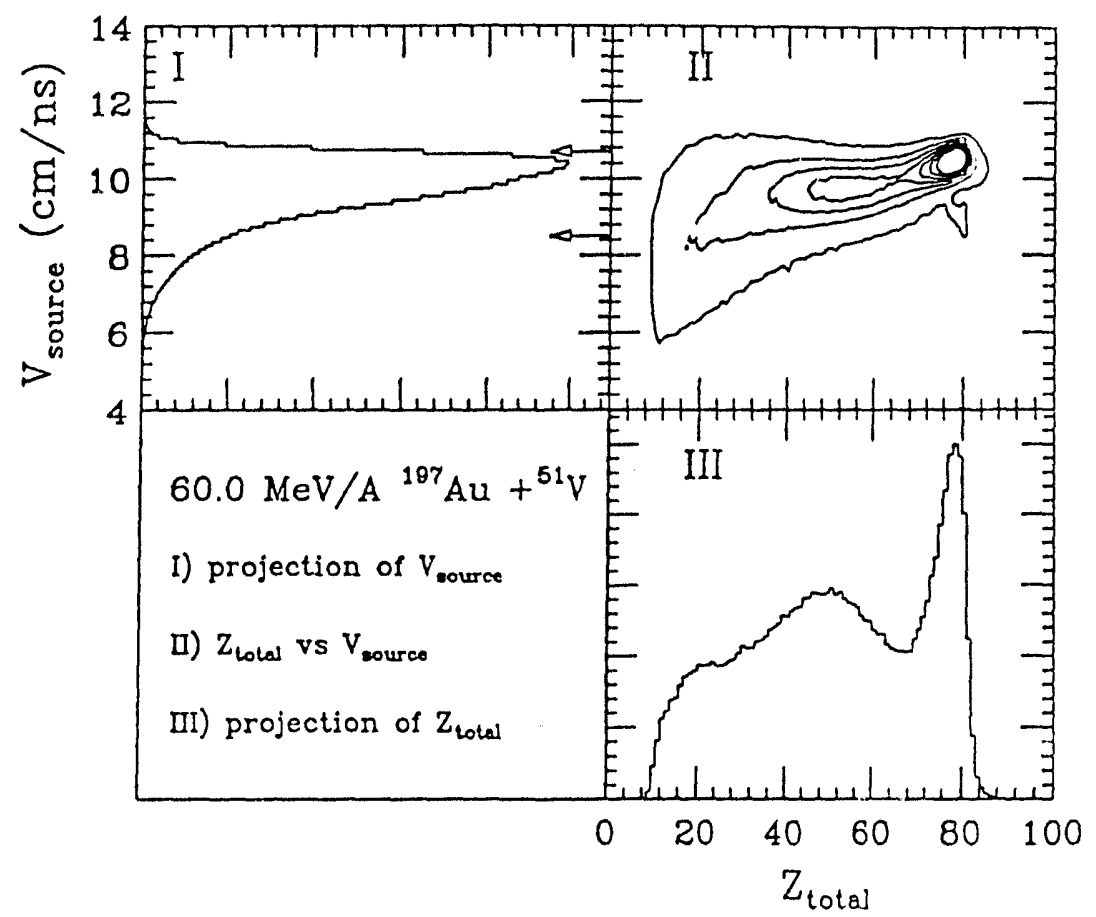

Figure 5.9c

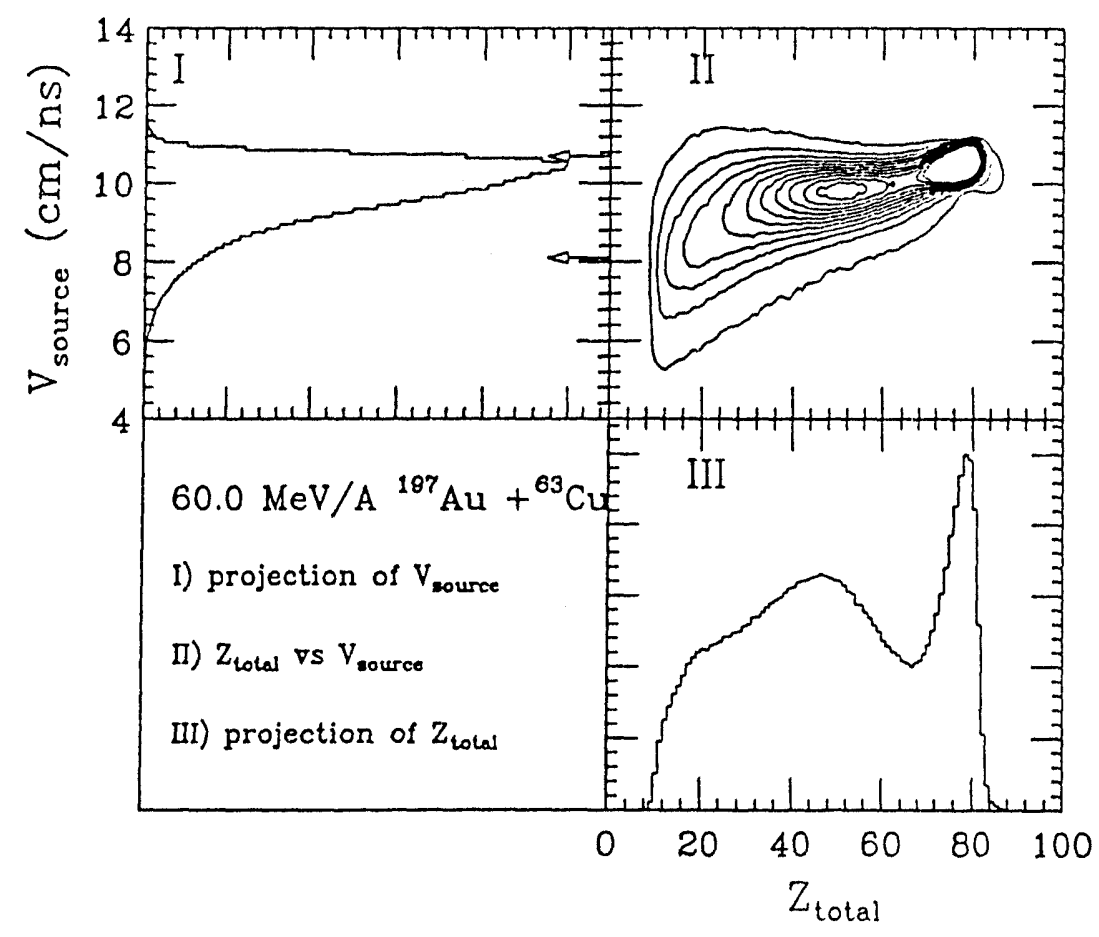

Figure 5.9d 


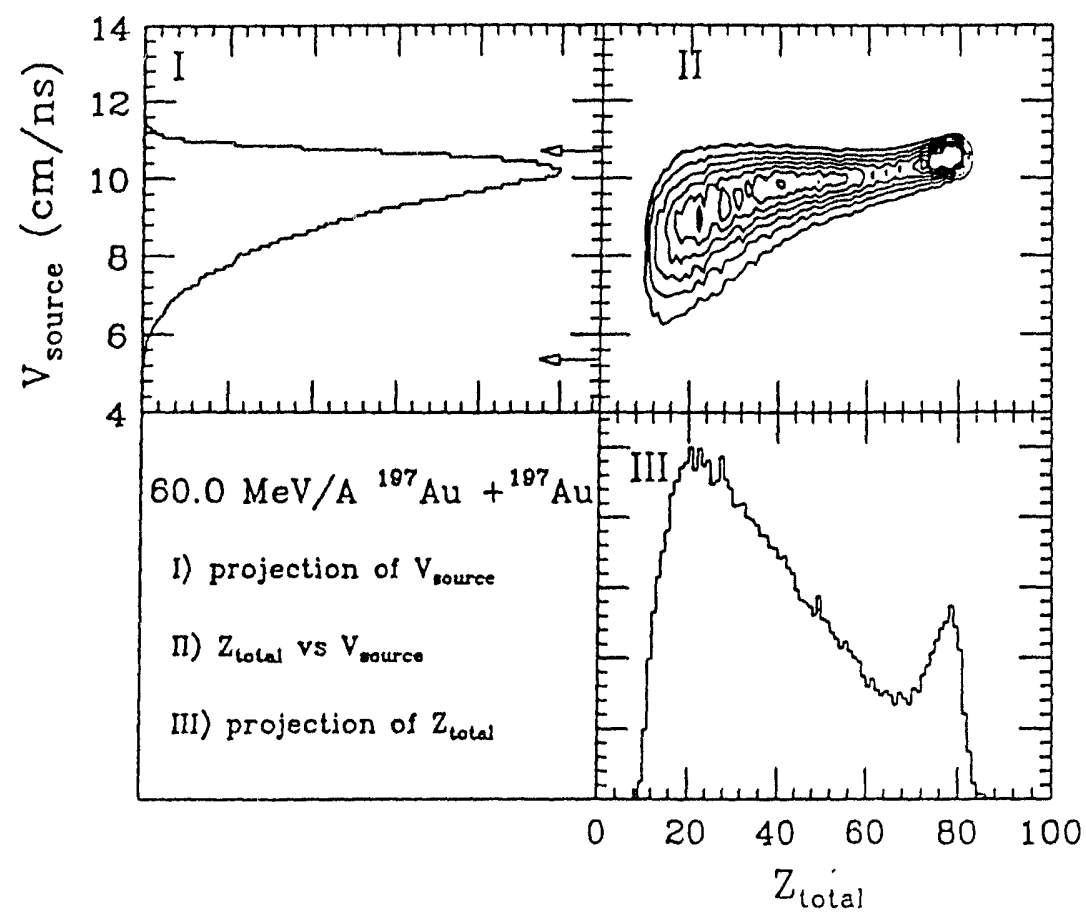

Figure 5.9e

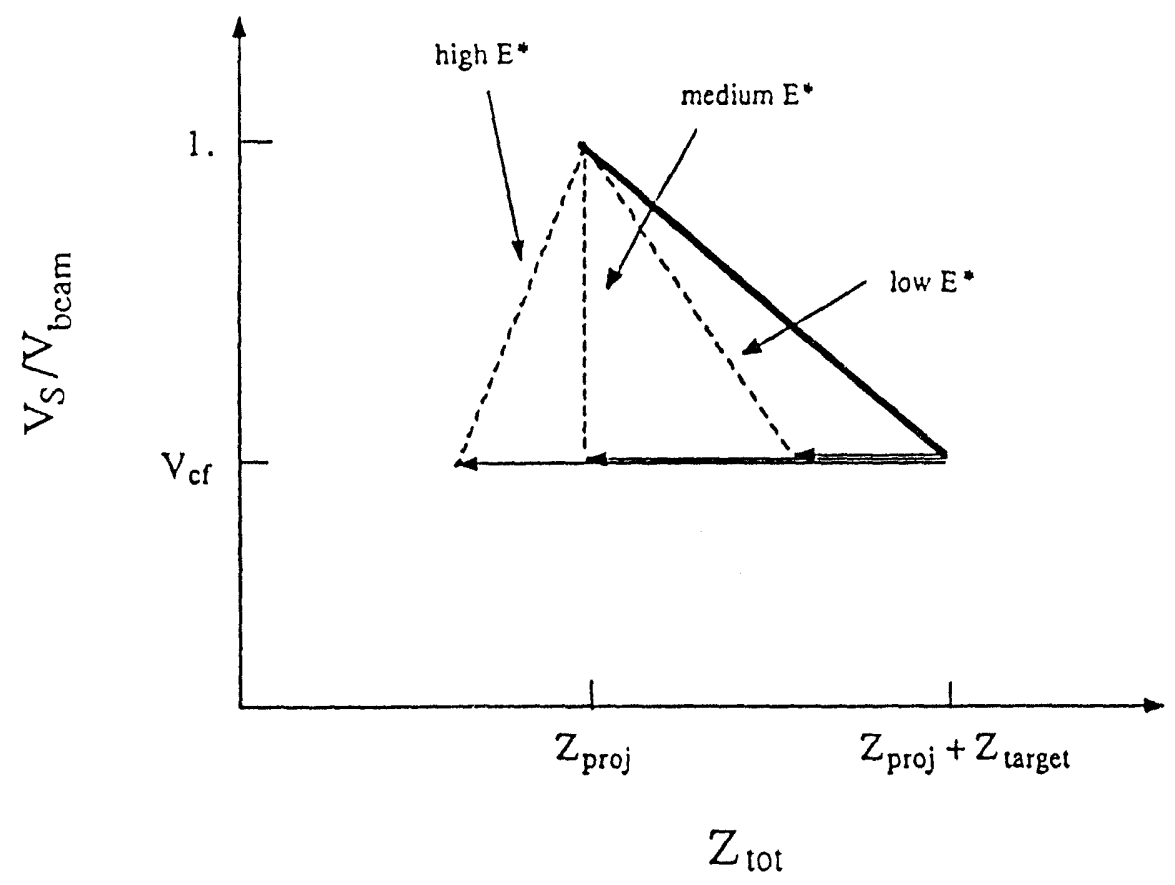

Figure 5.10 


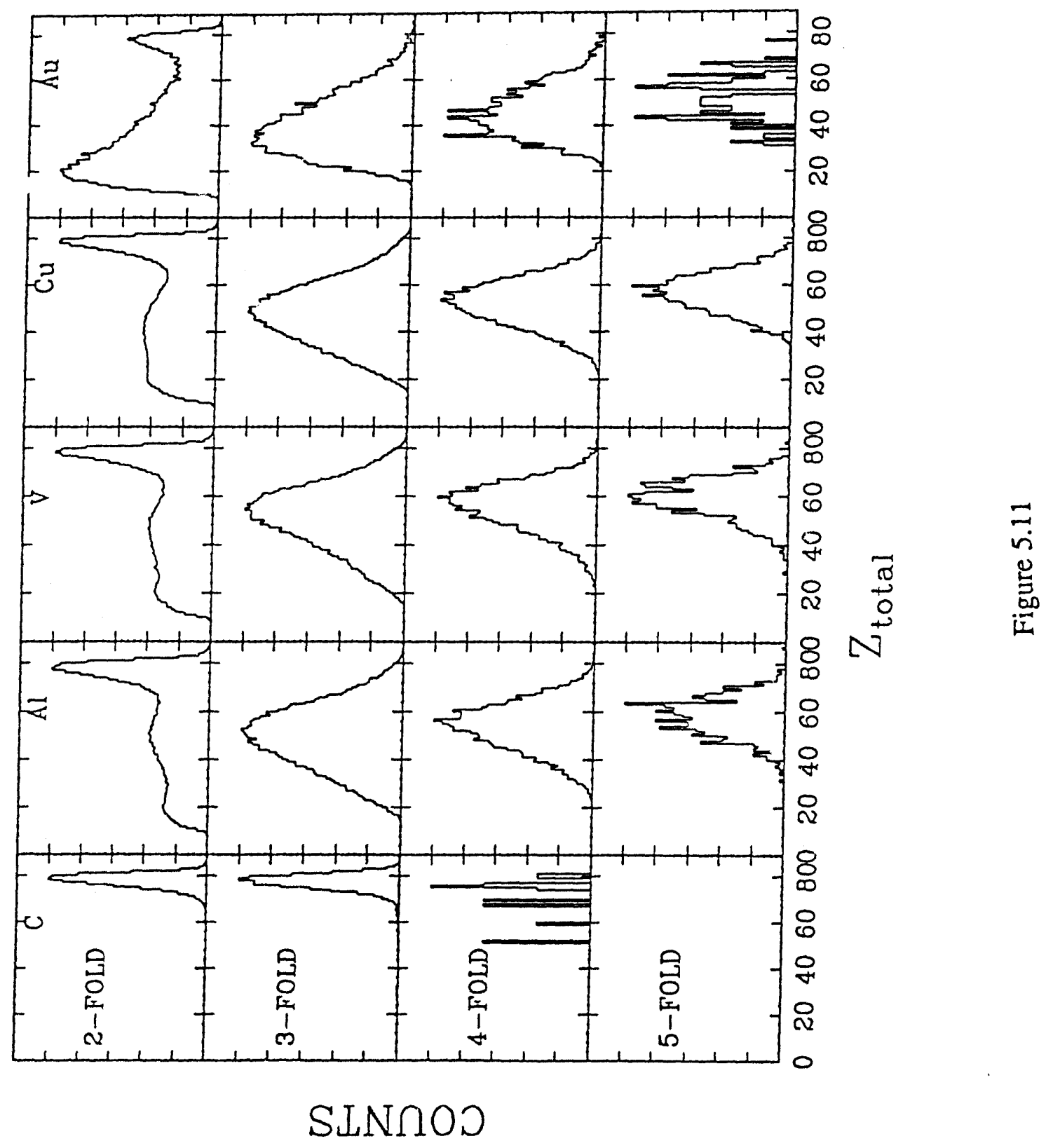




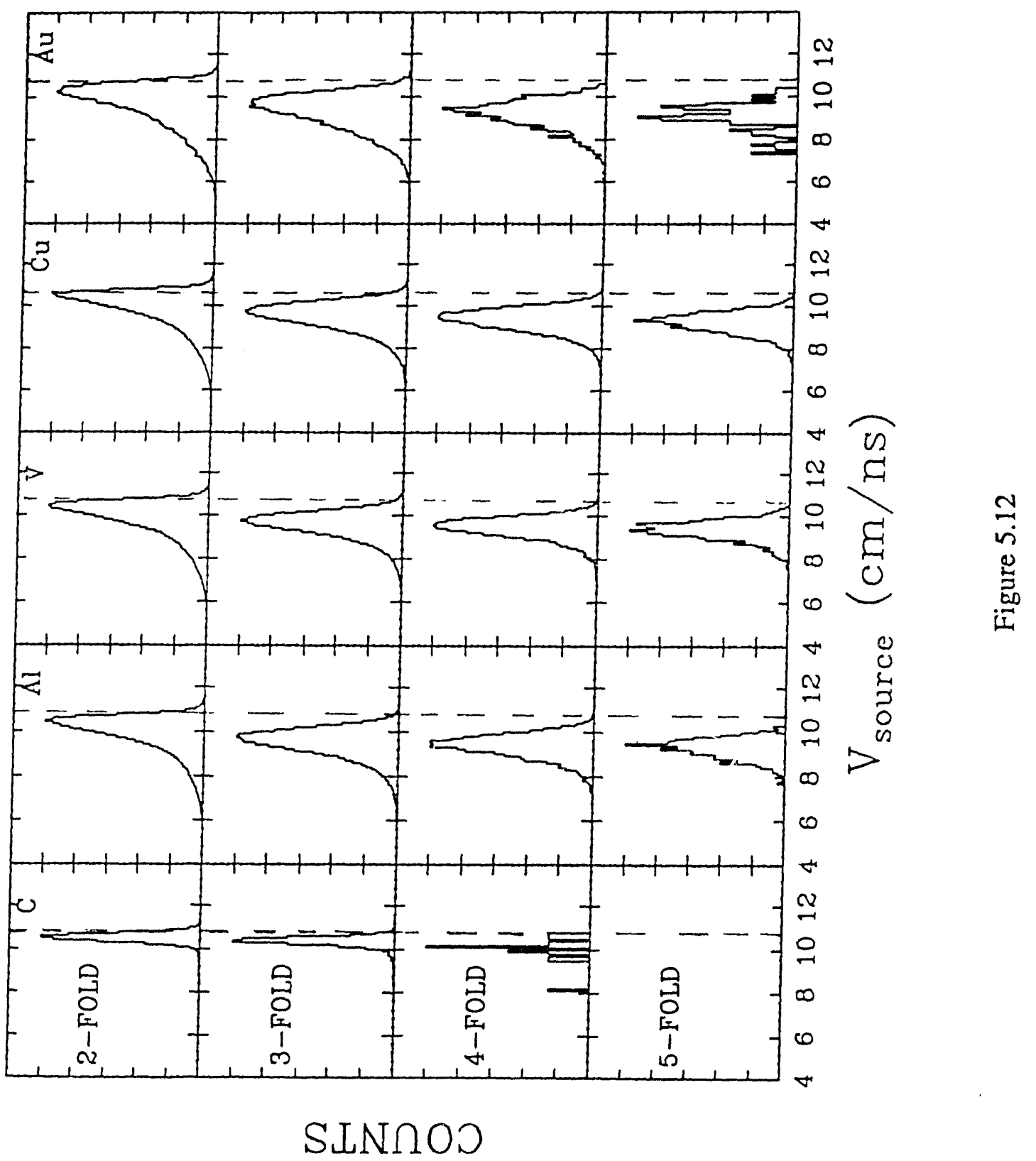




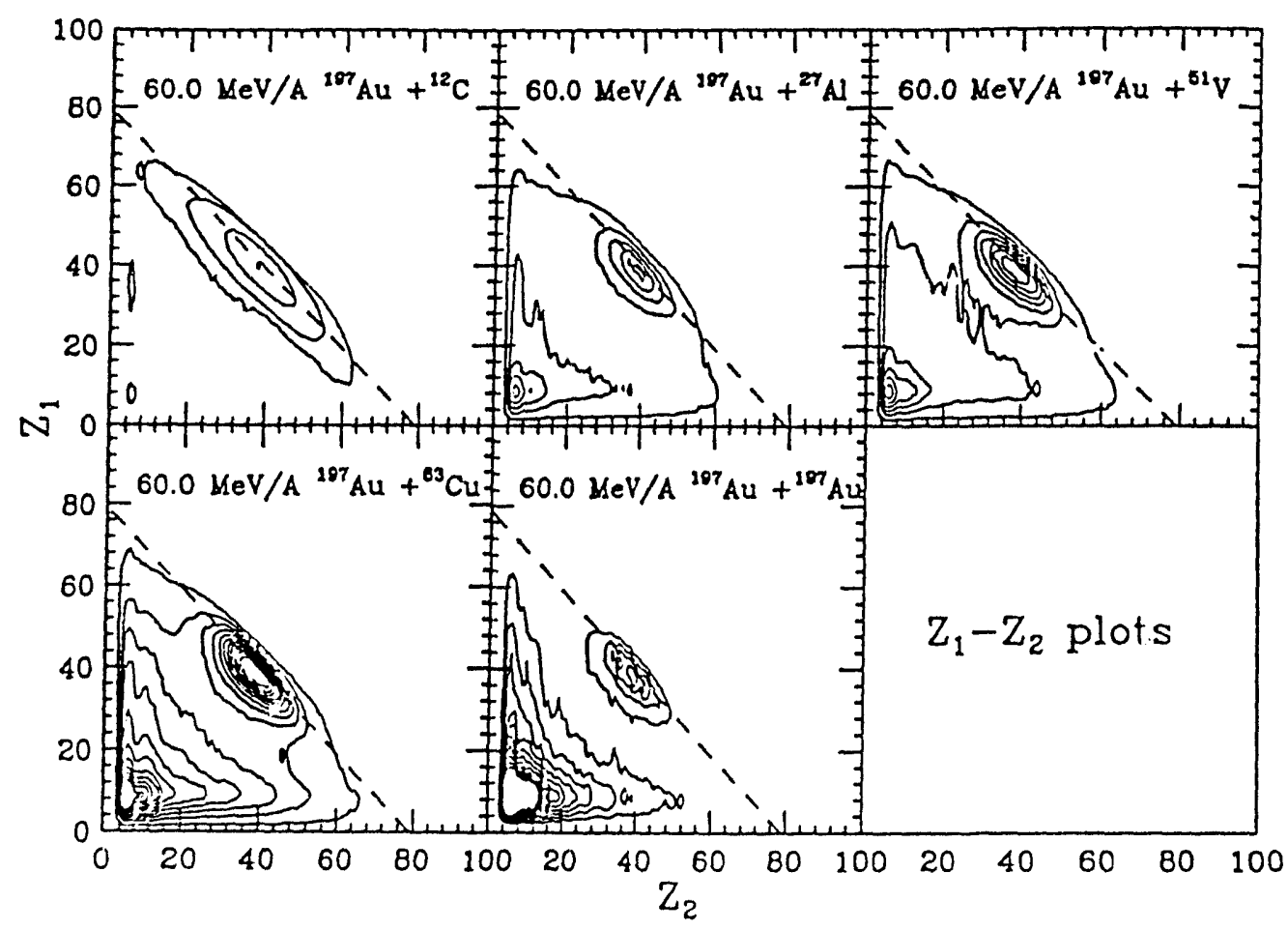

Figure 5.13 


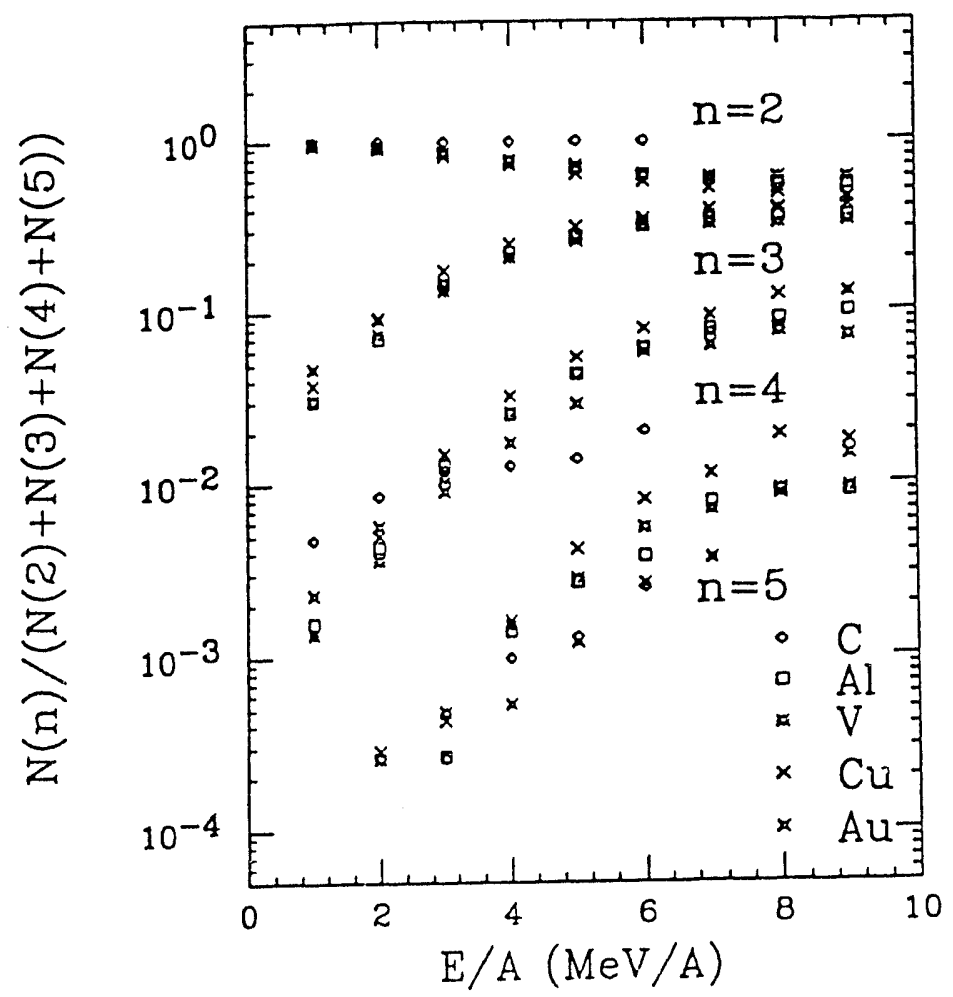

Figure 5.14

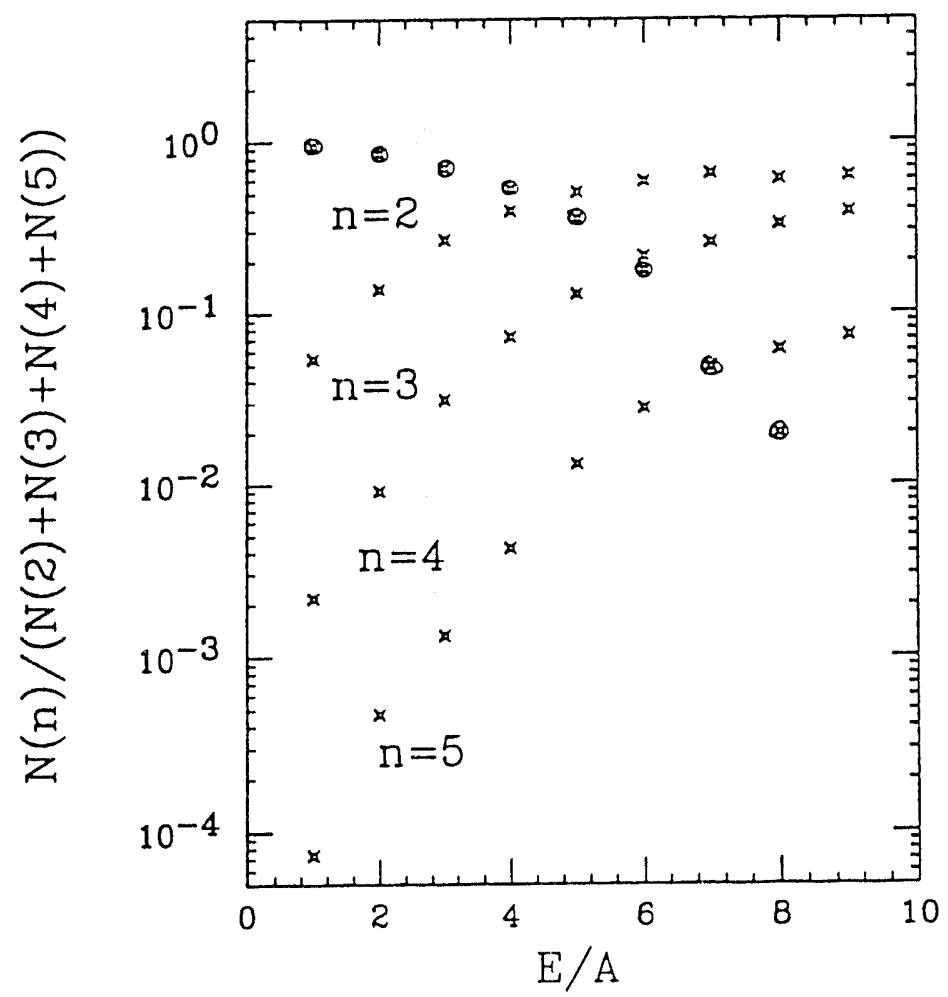

Figure 5.15 


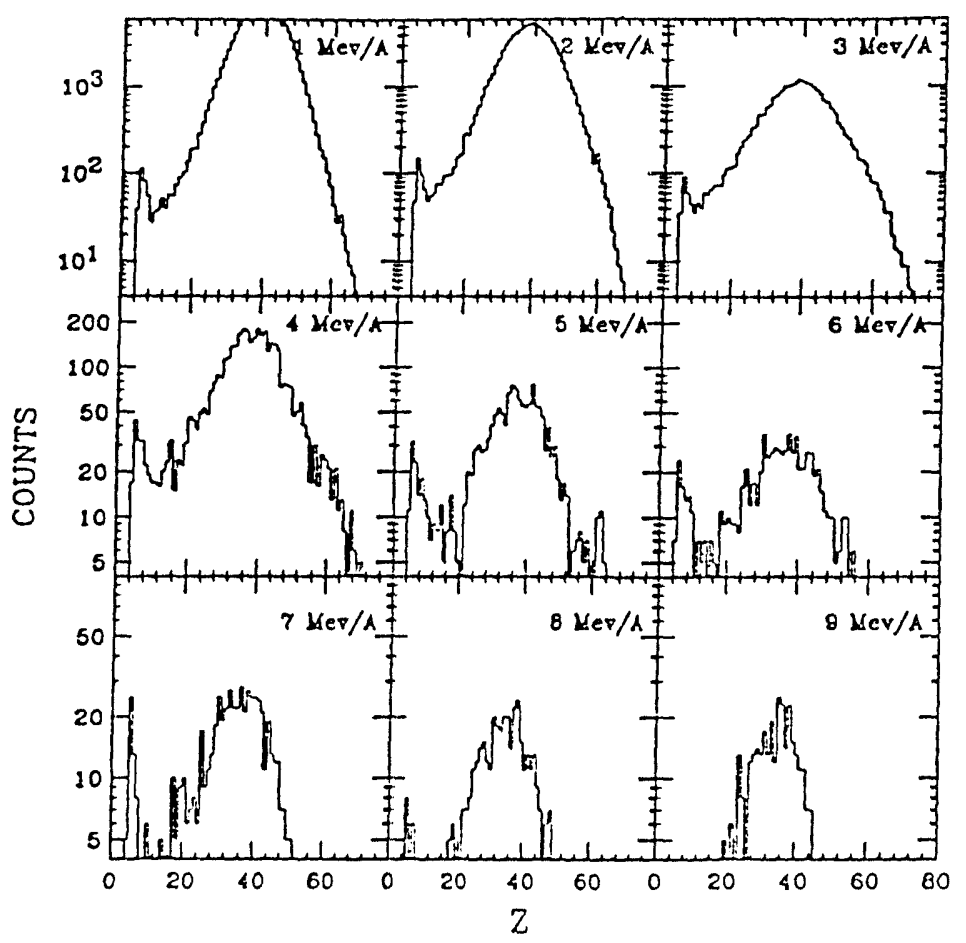

Figure 5.16a

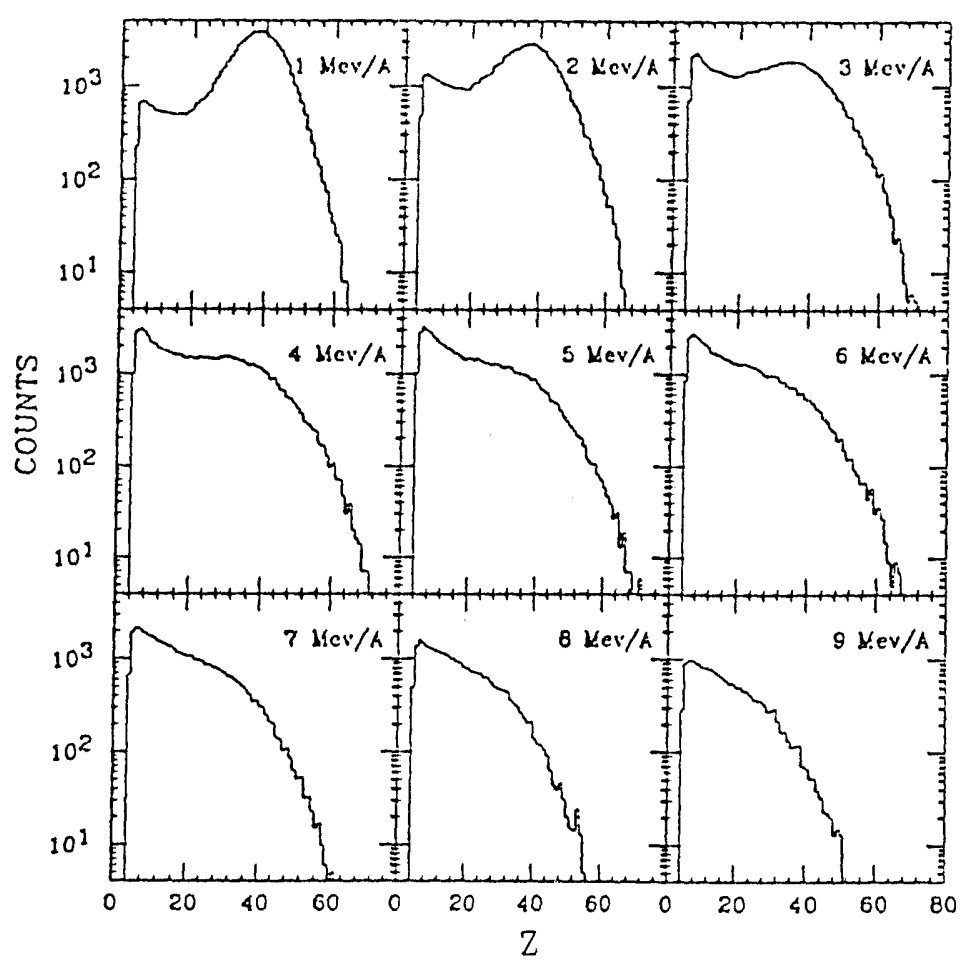

Figure 5.16b 


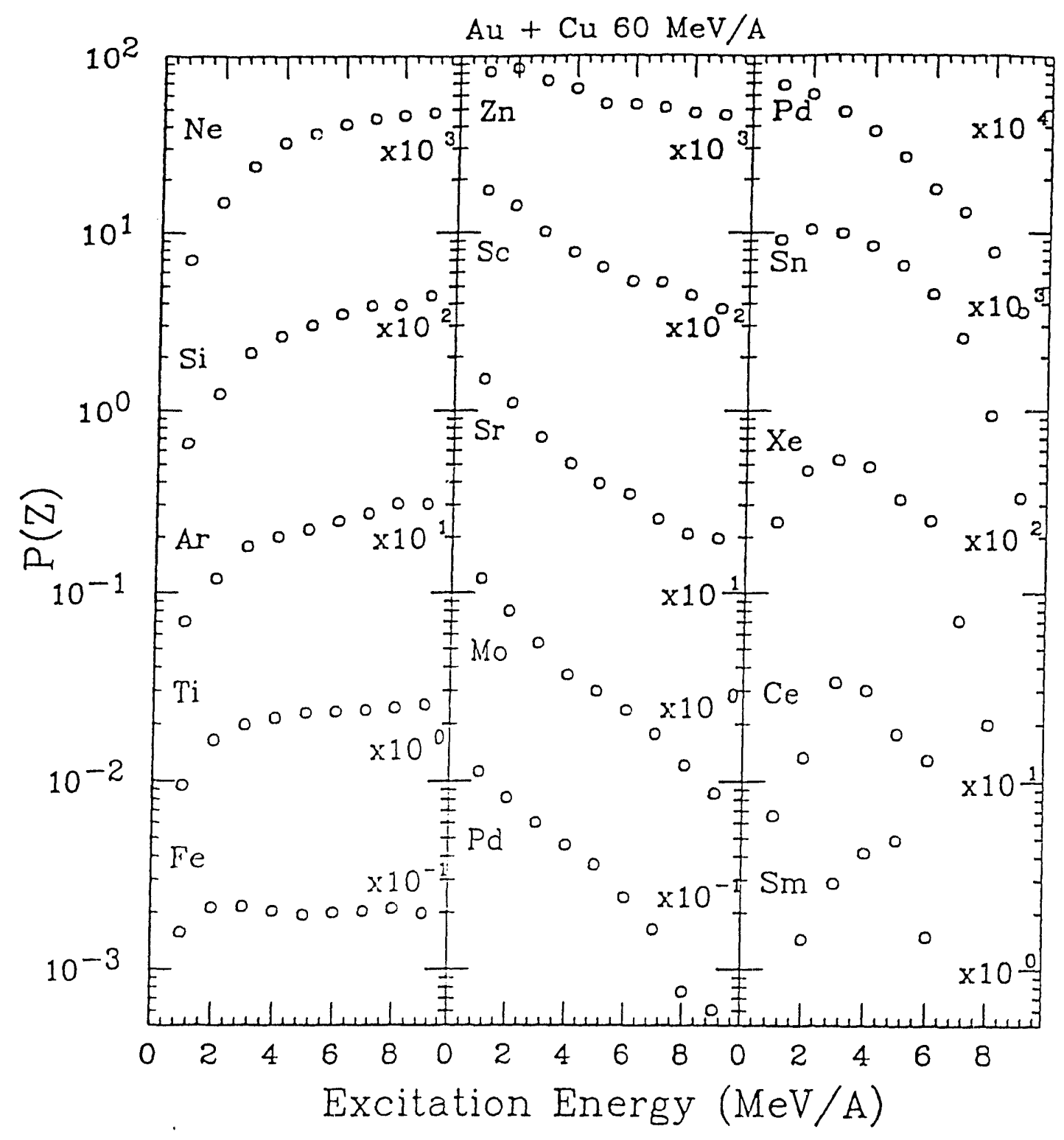

Figure 5.17 


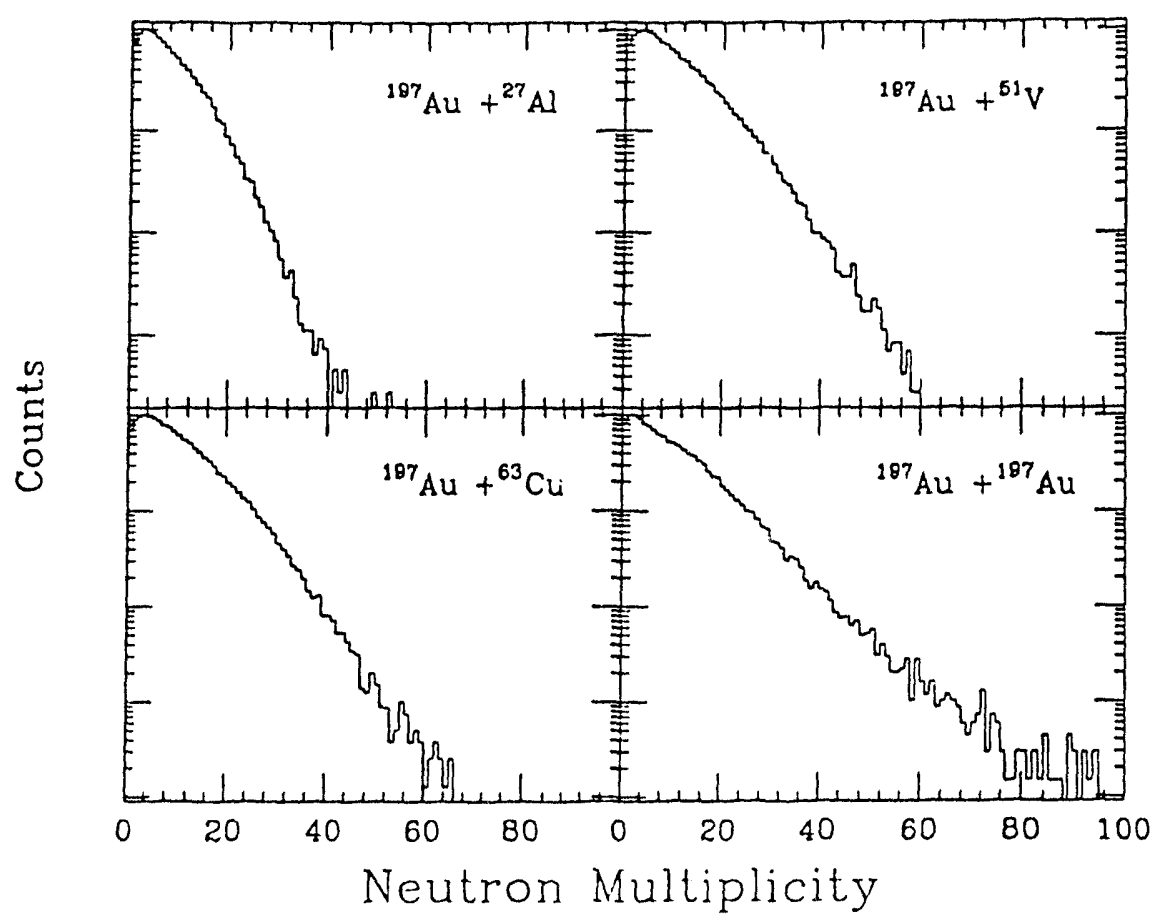

Figure 5.18

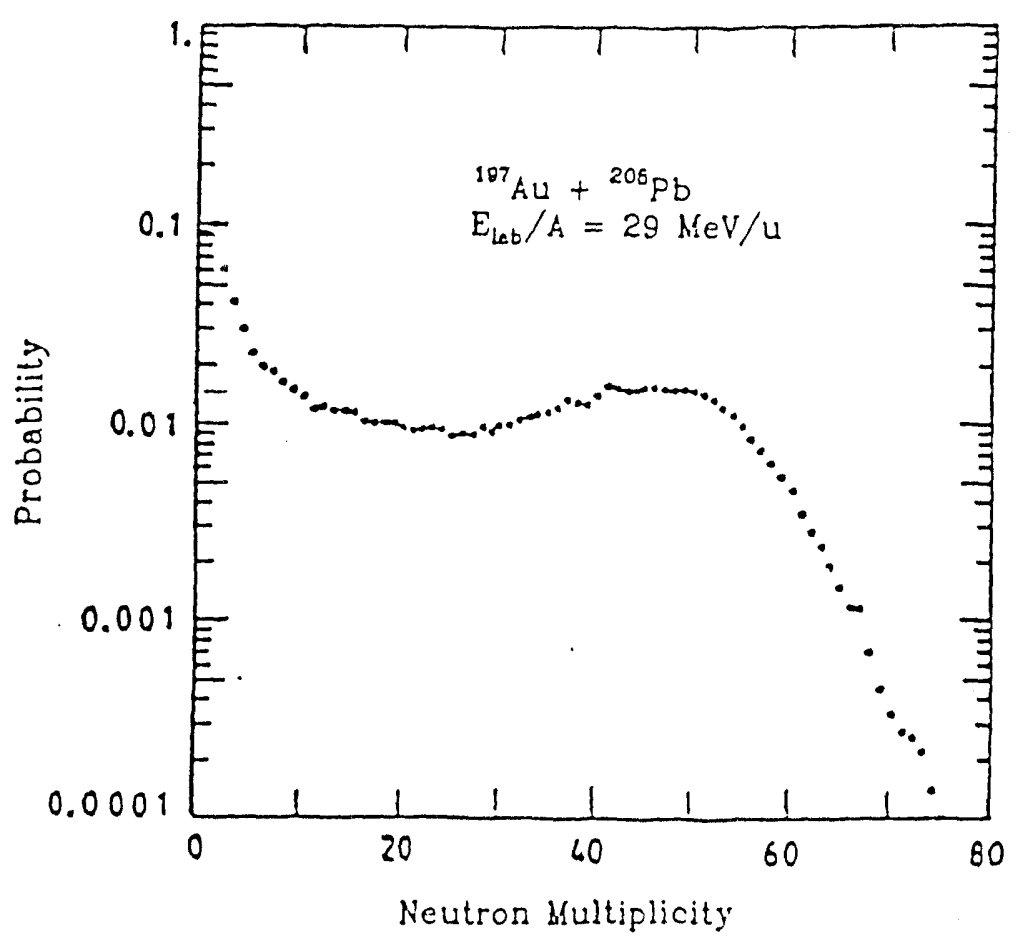

Figure 5.19 


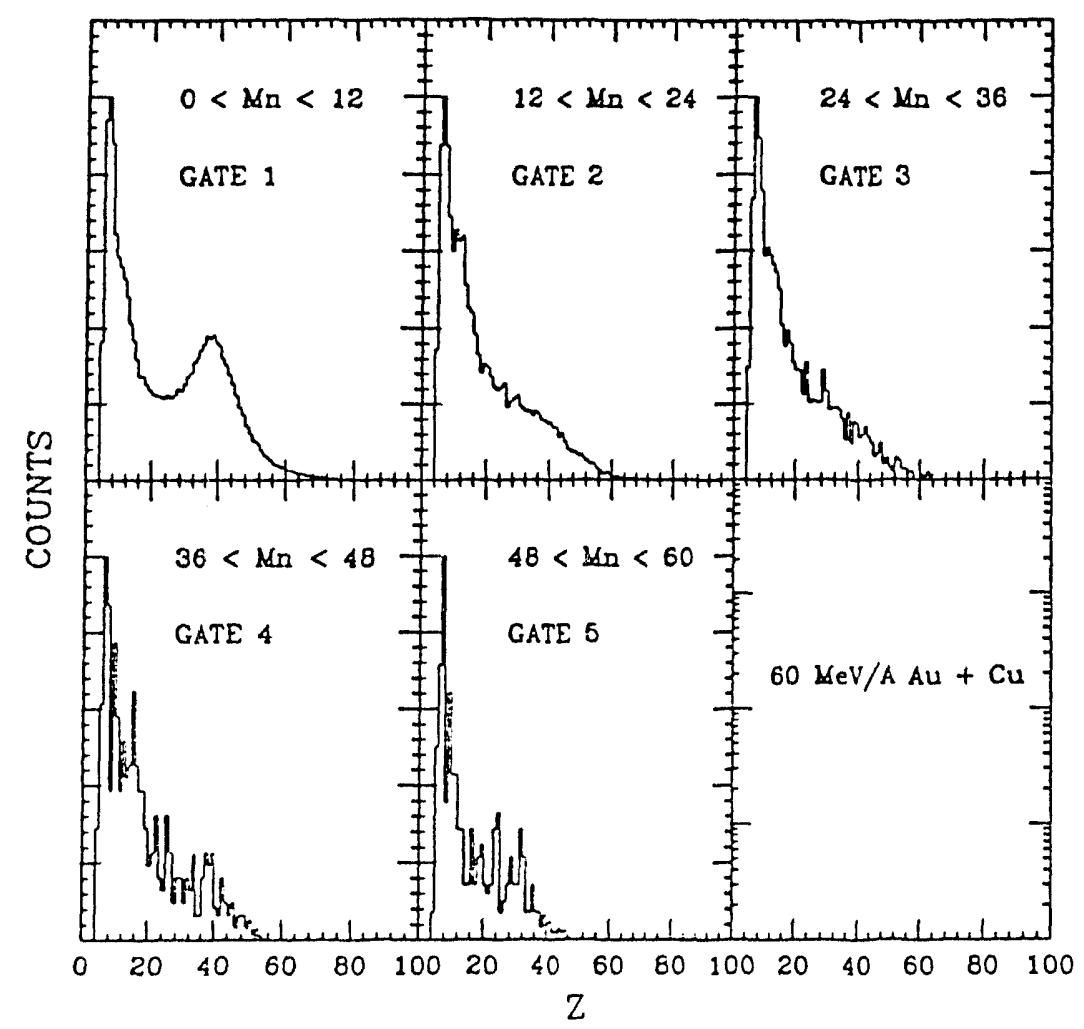

Figure 5.20 


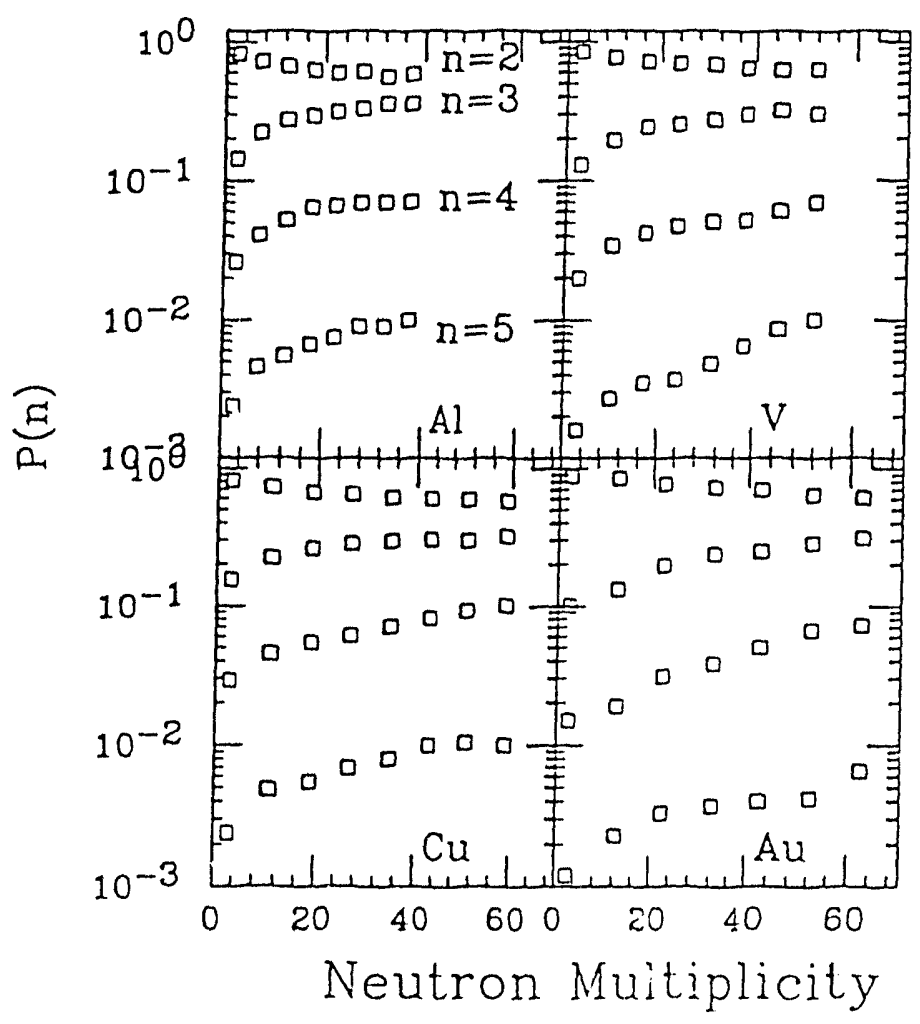

Figure 5.21

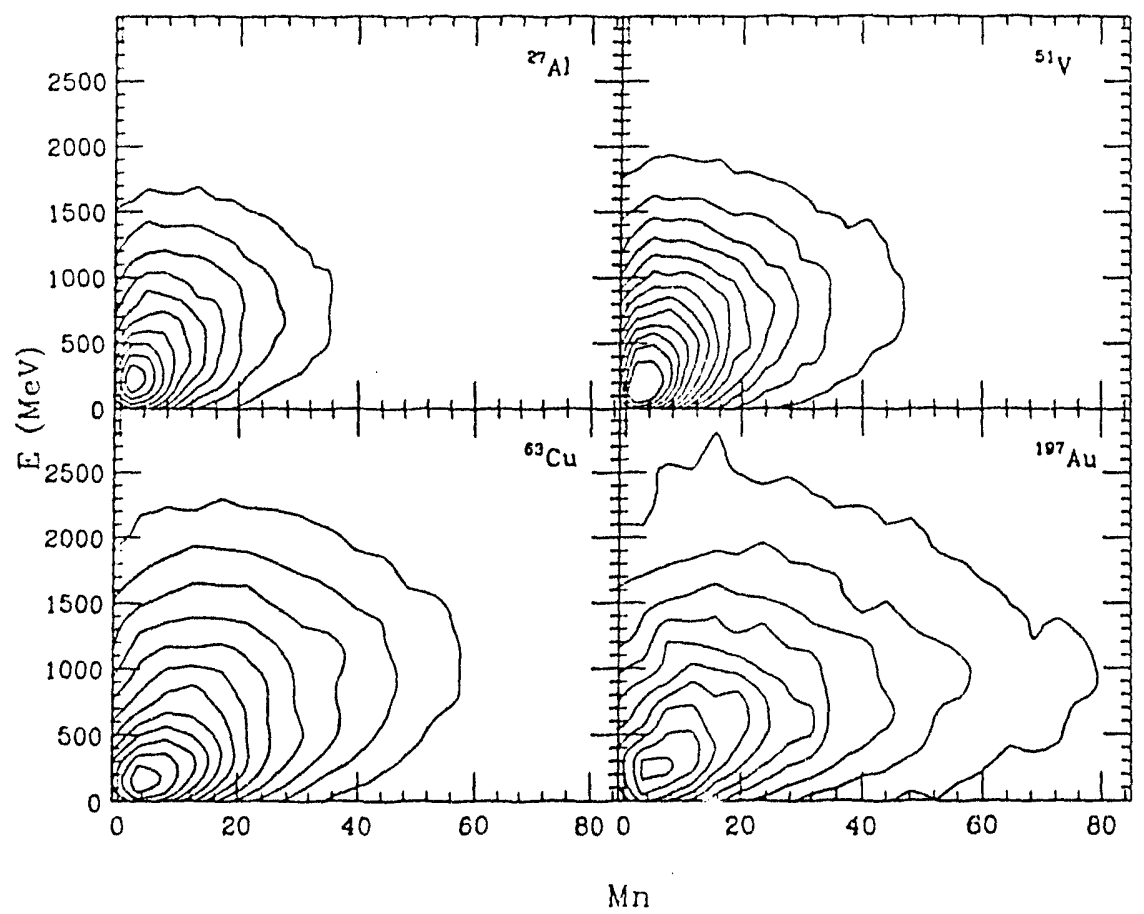

Figure 5.22 


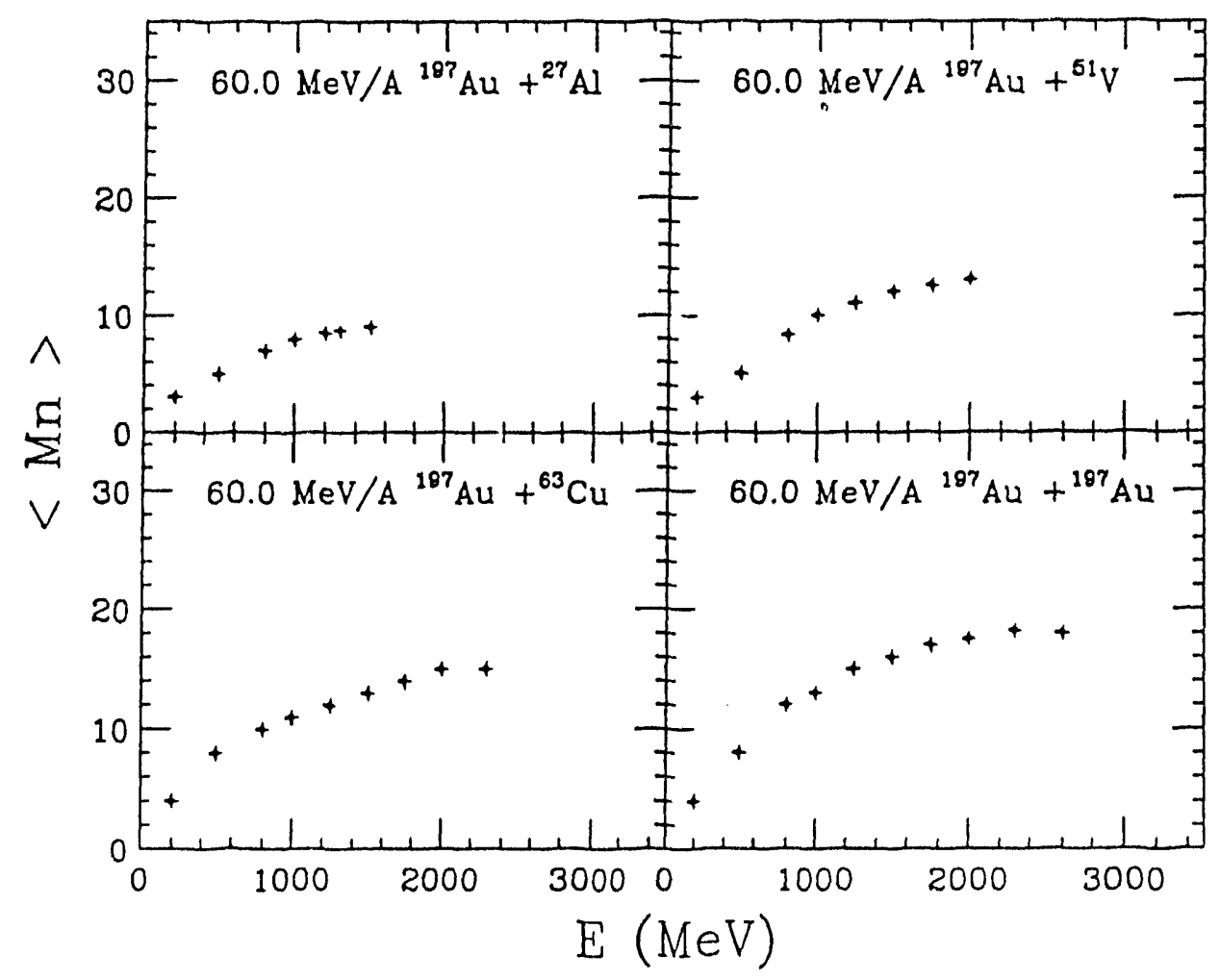

Figure 5.23

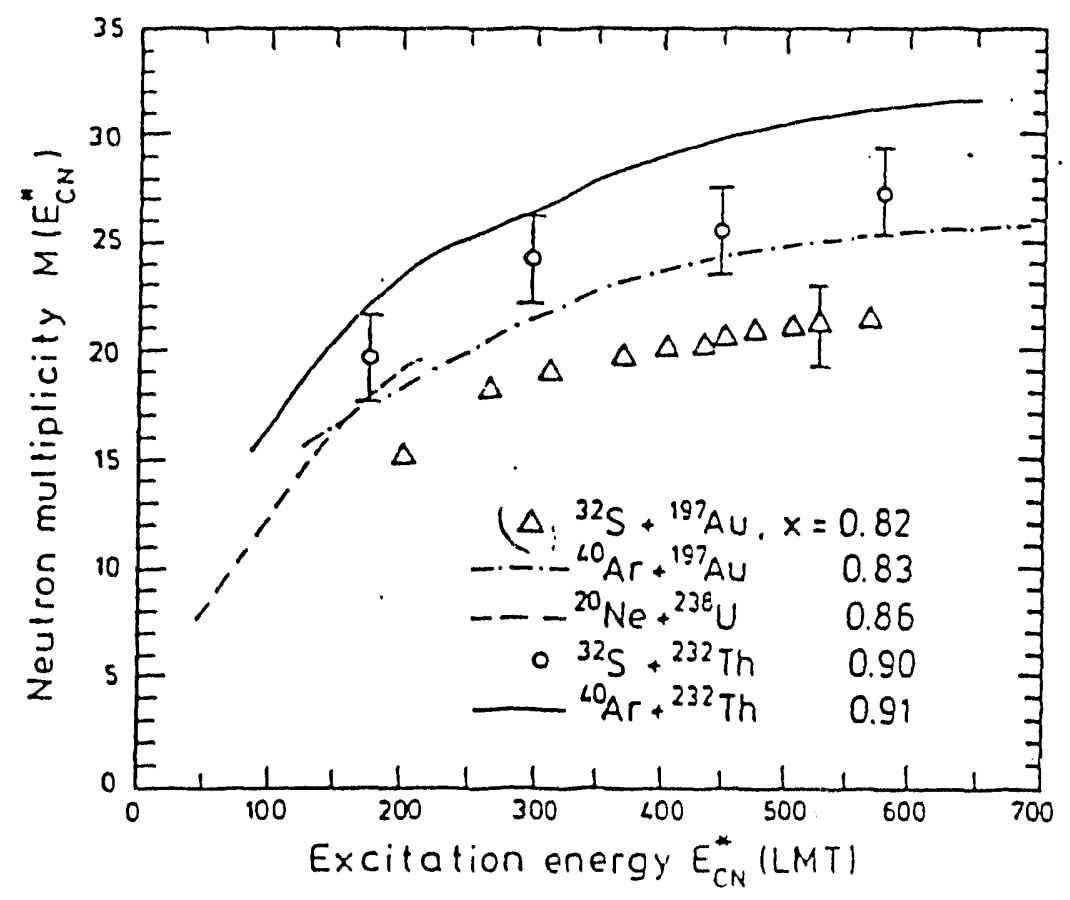

Figure 5.24 

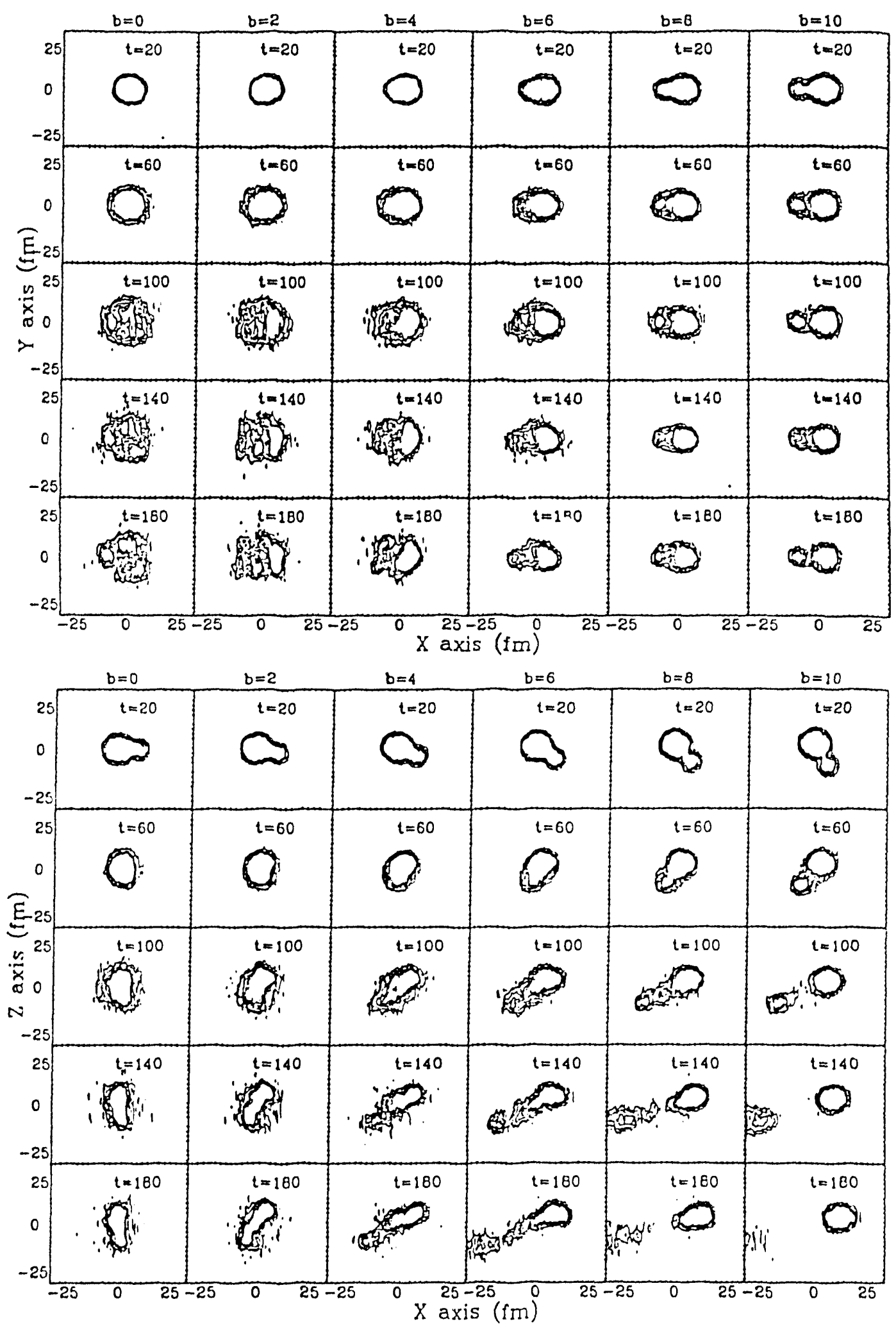

Figure 6.1 


$$
\mathrm{Au}+\mathrm{Al} \text { at } \mathrm{b}=0
$$

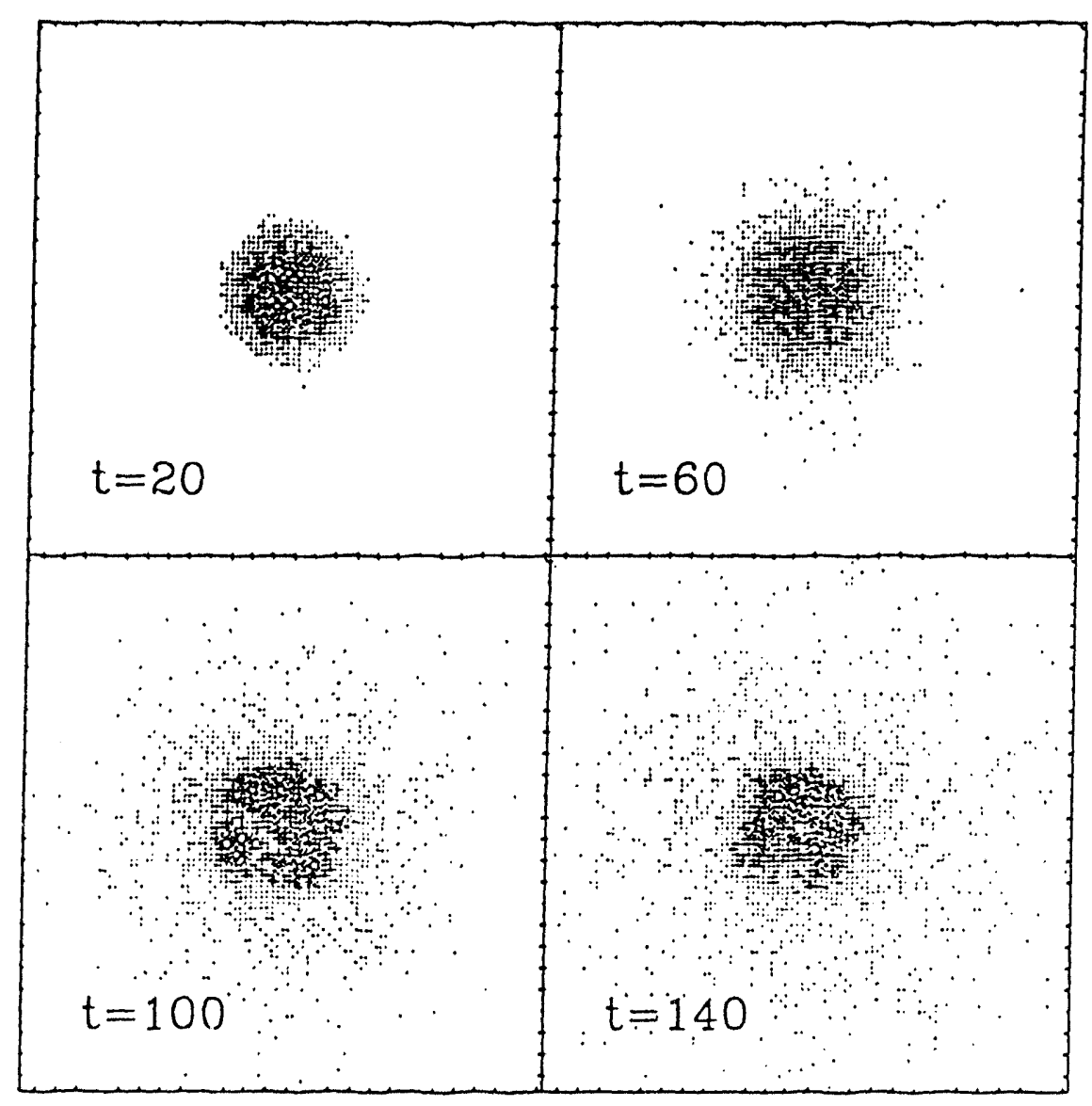

Figure 6.2 


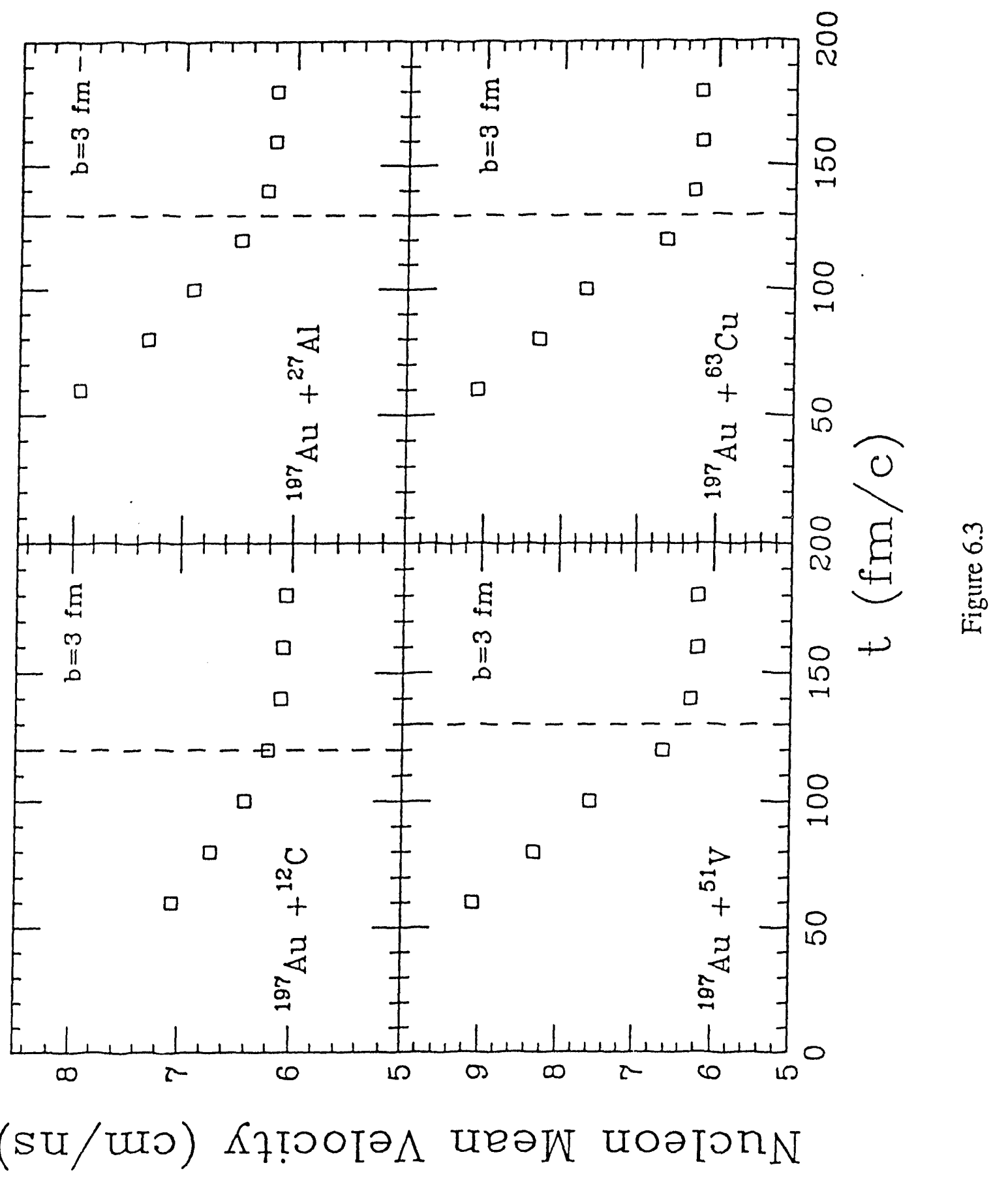




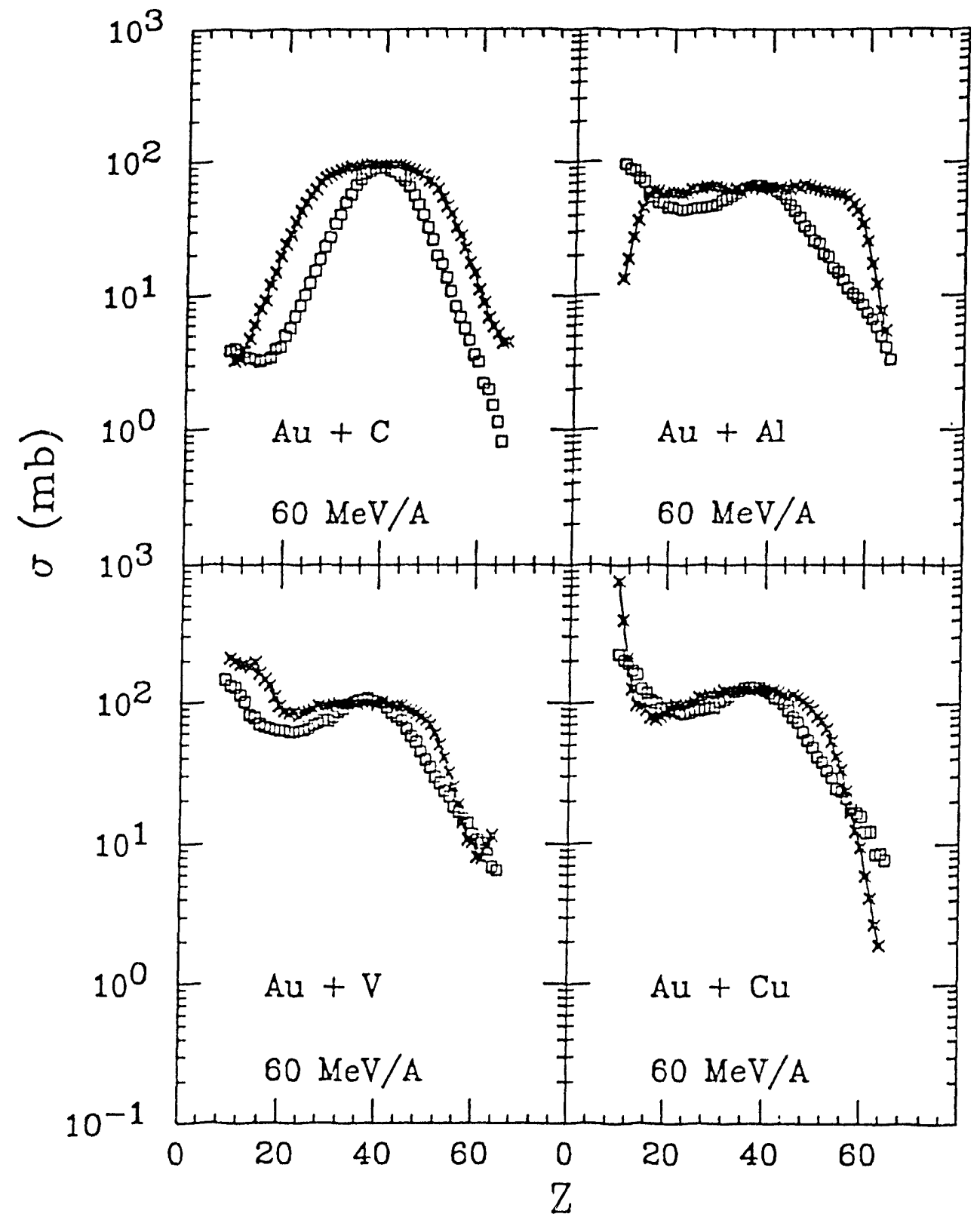

Figure 6.4 


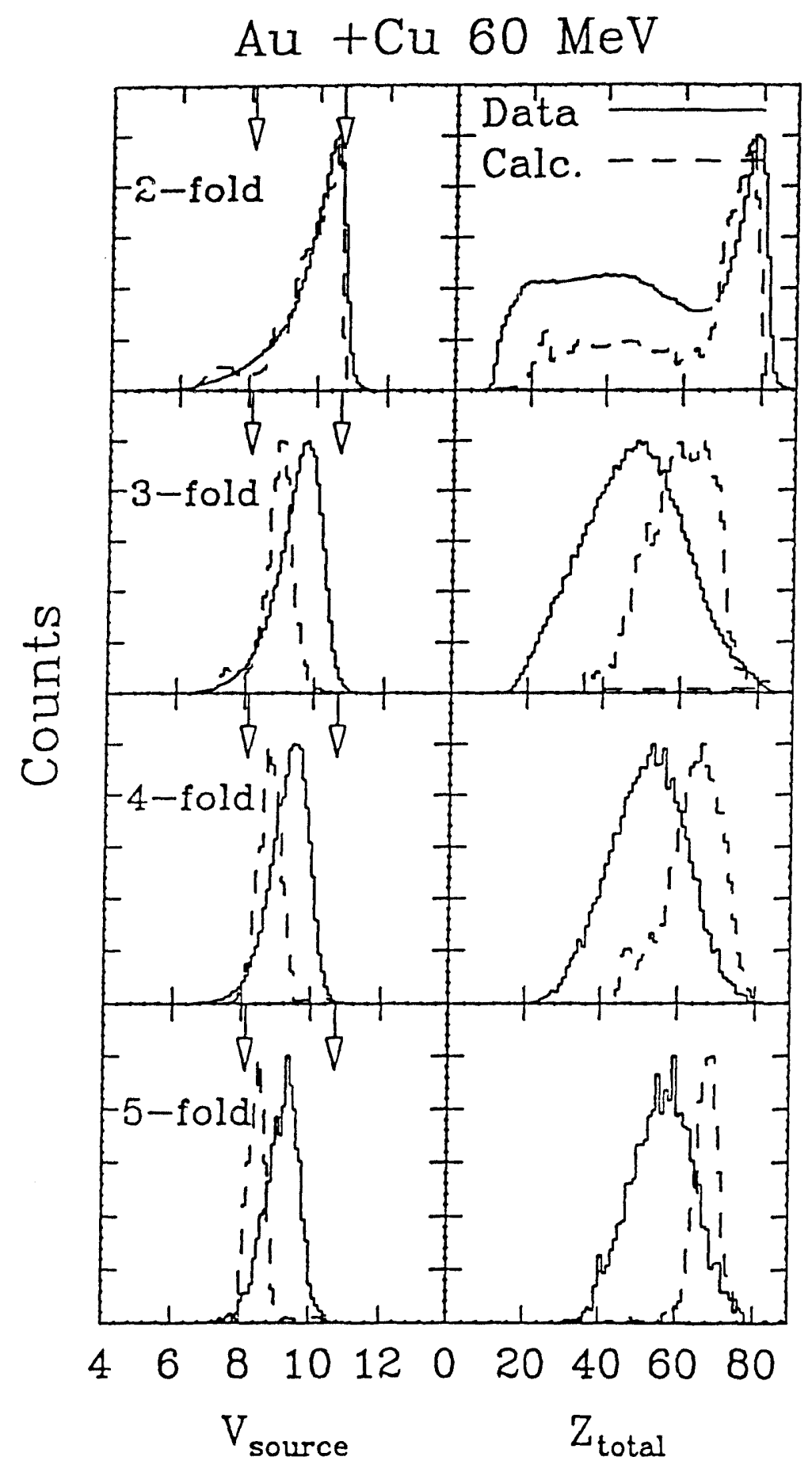

Figure 6.5 


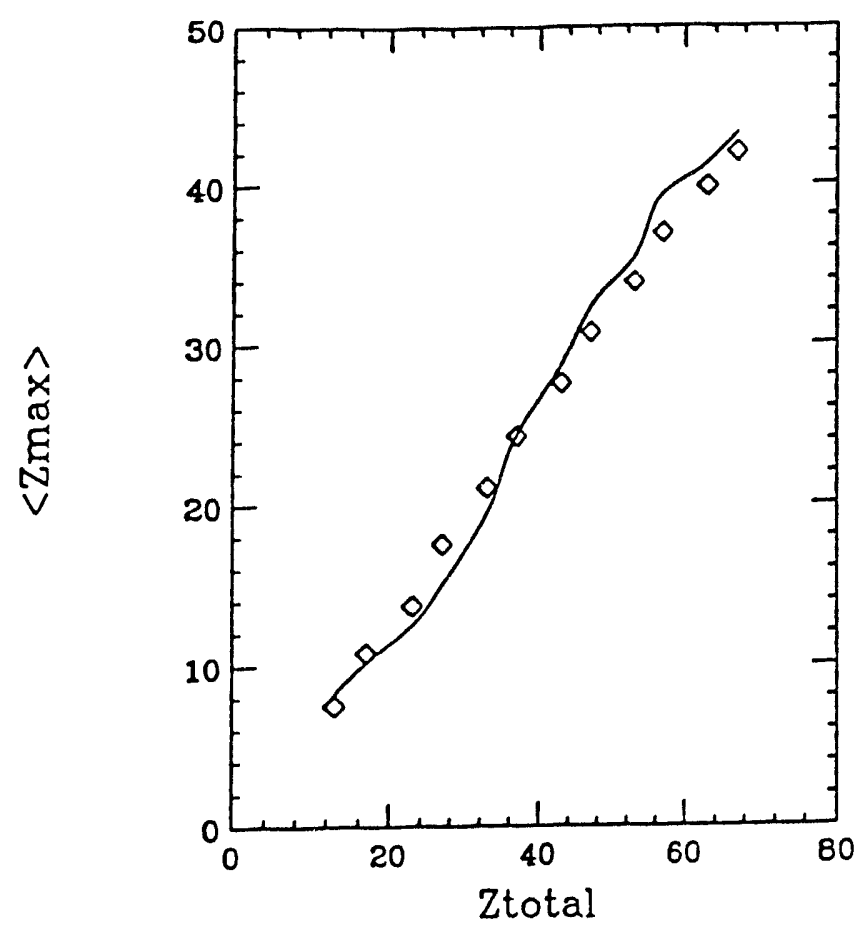

Figure 6.6

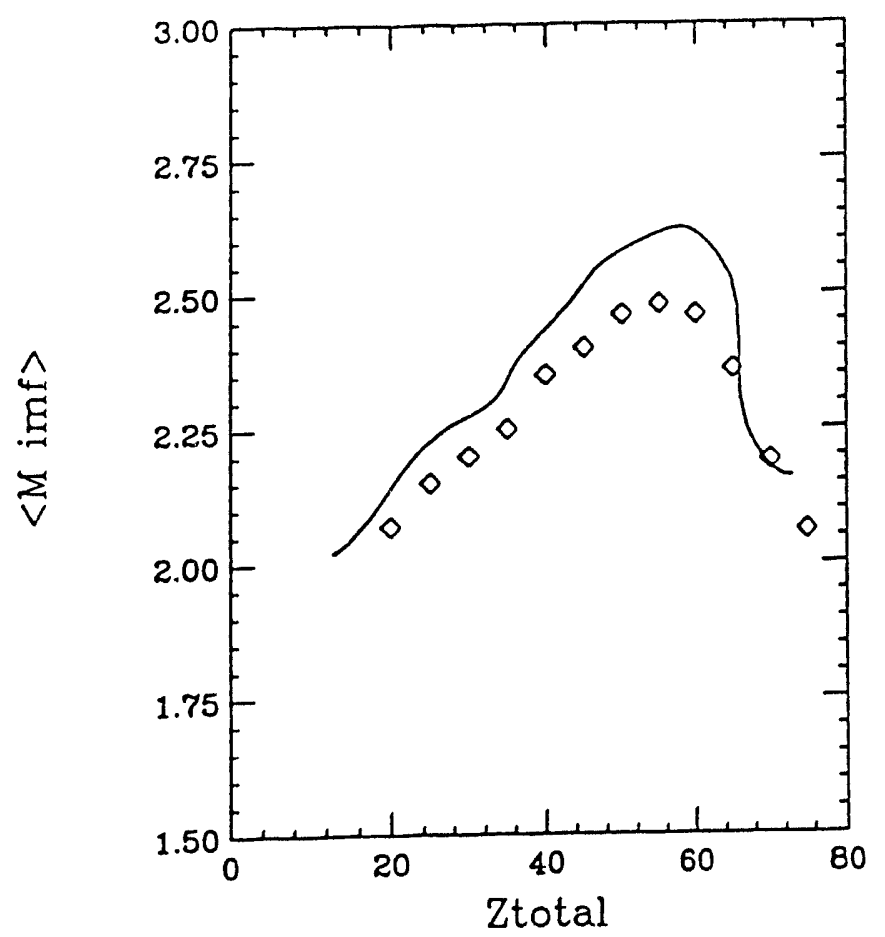

Figure 6.7 


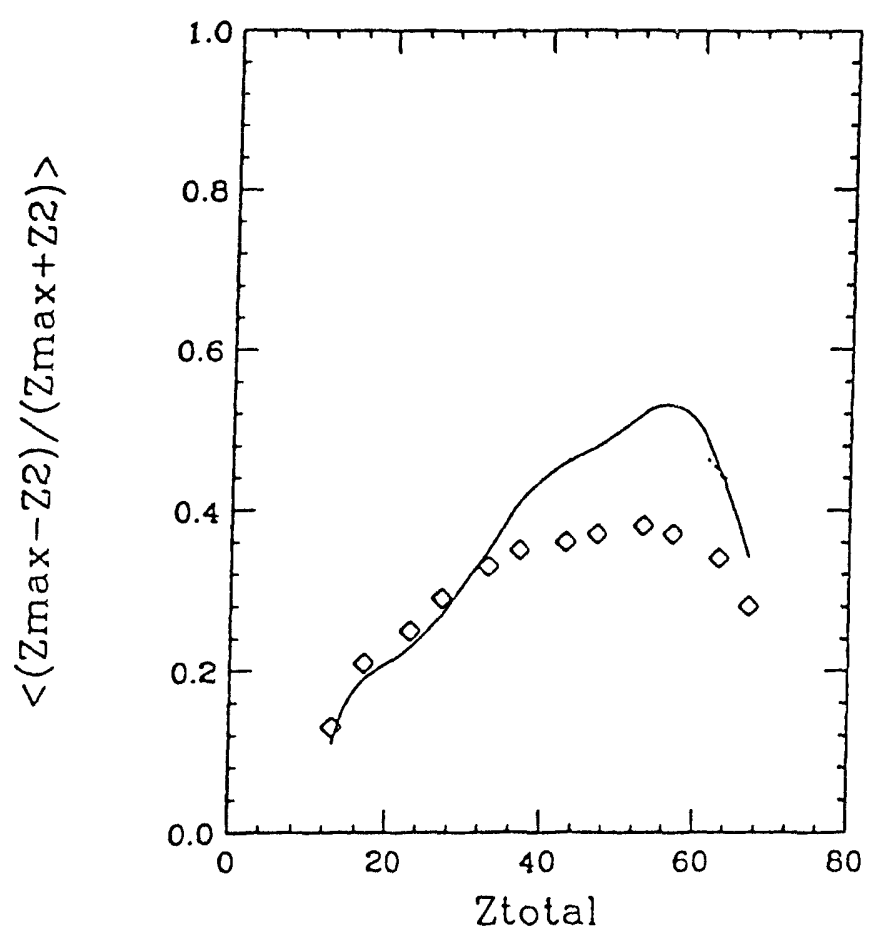

Figure 6.8

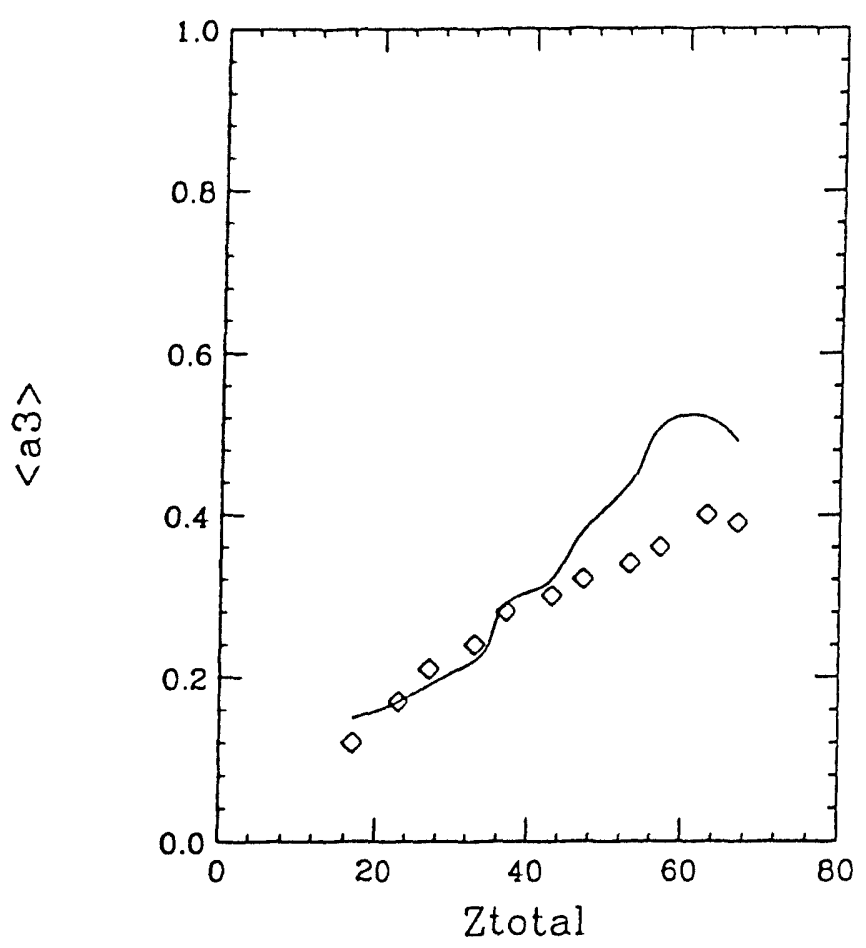

Figure 6.9 


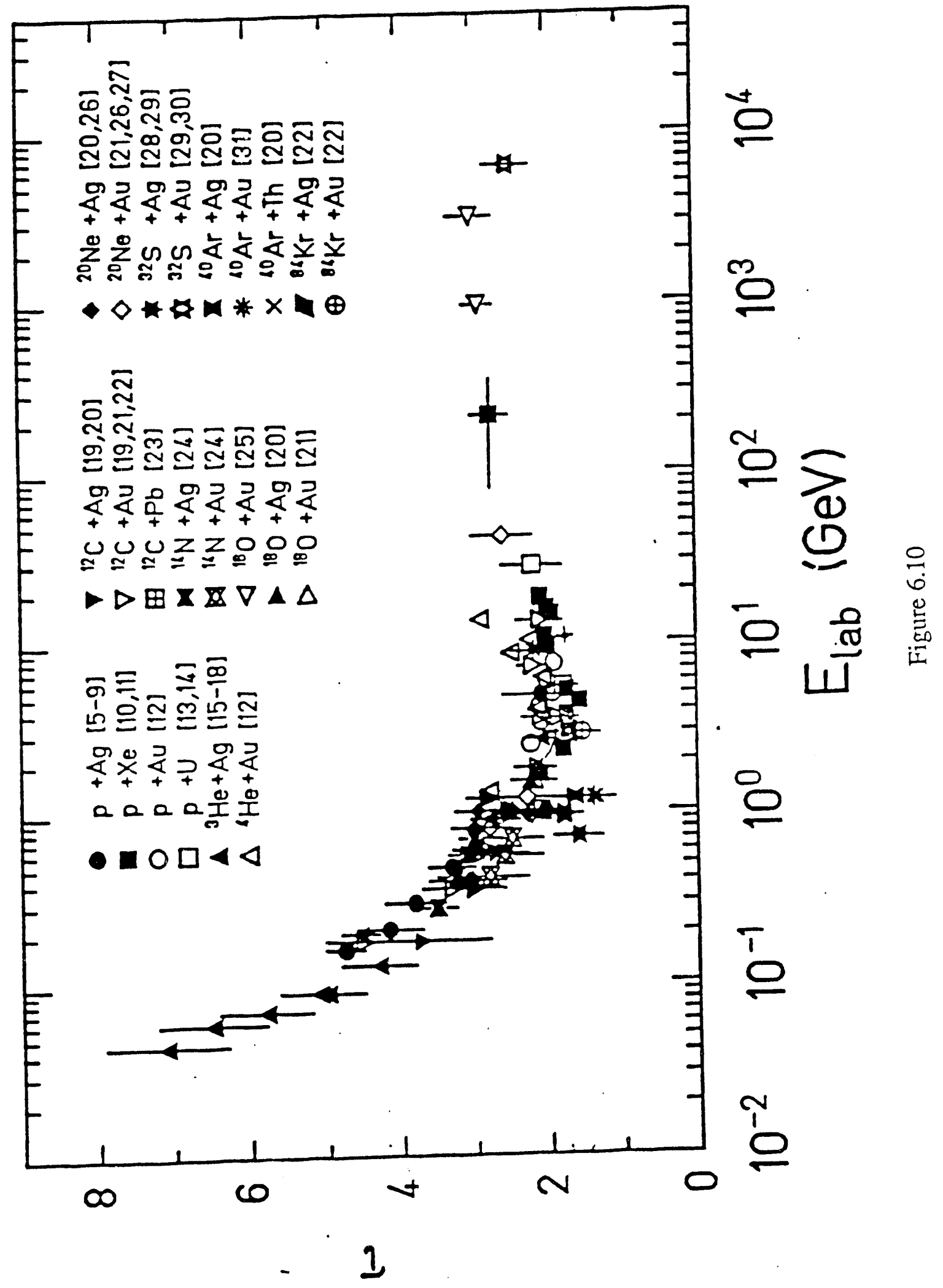




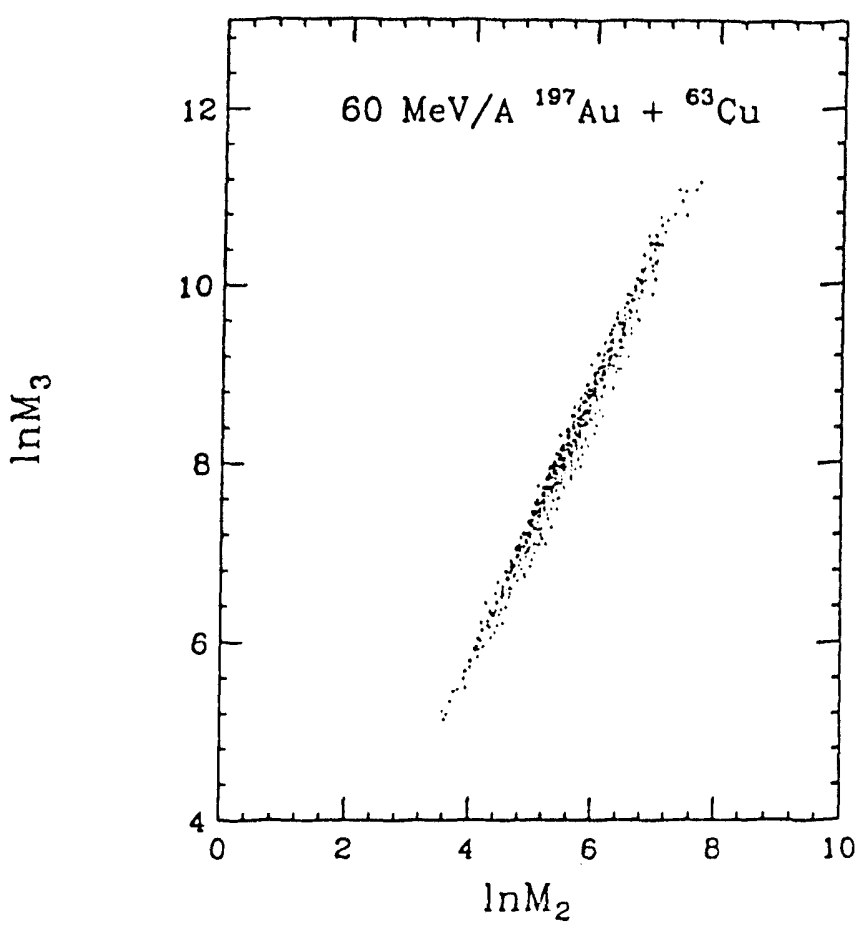

Figure 6.11

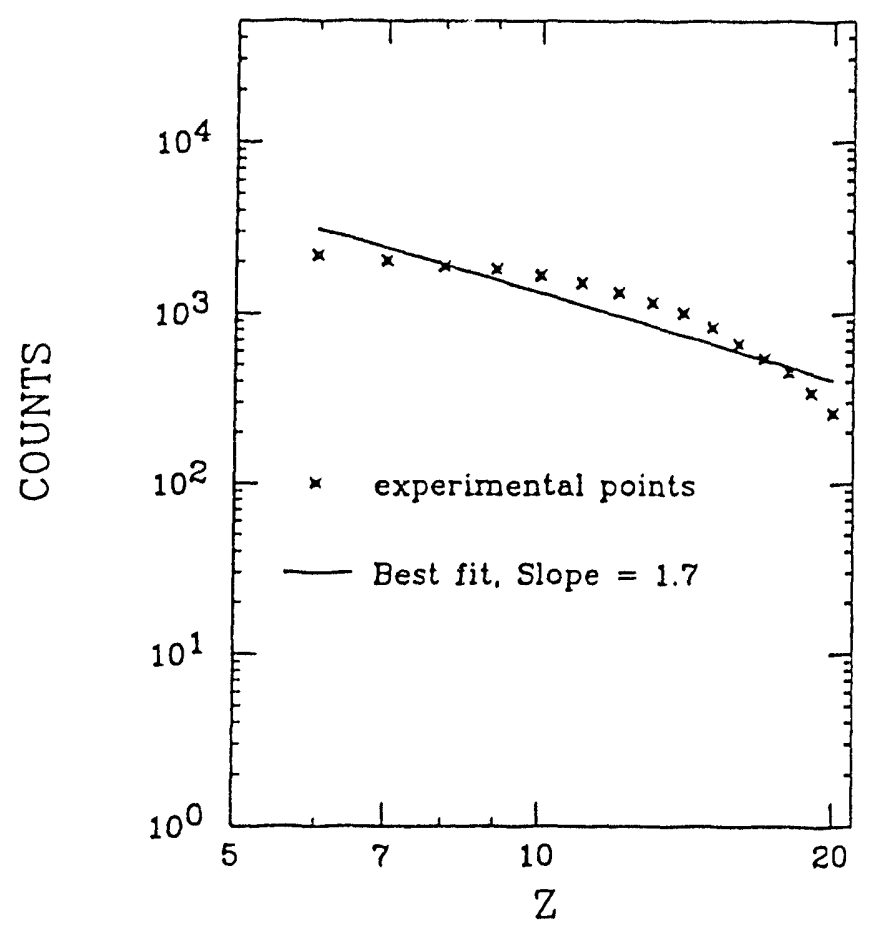

Figure 6.12 


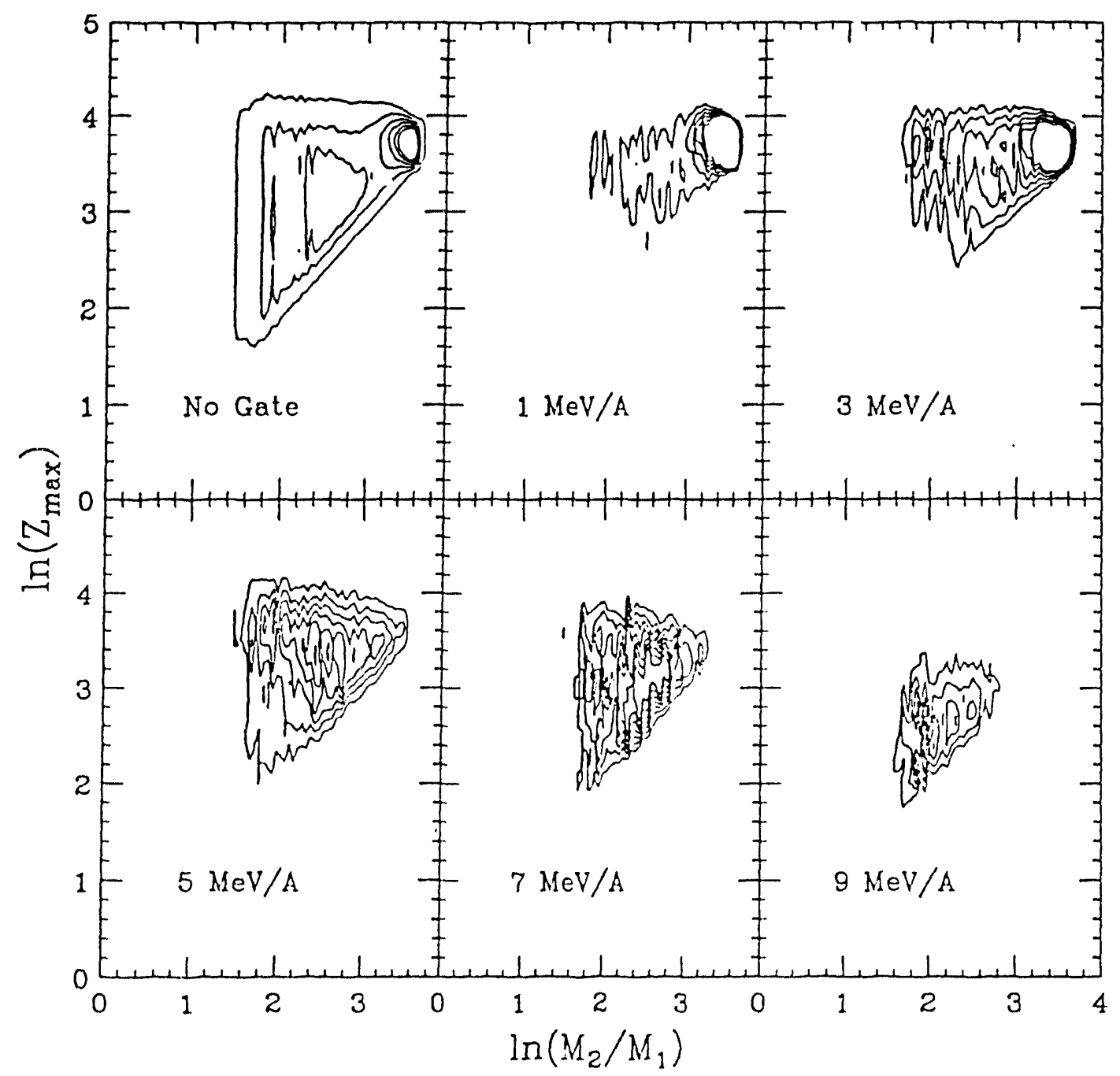

Figure 6.13 


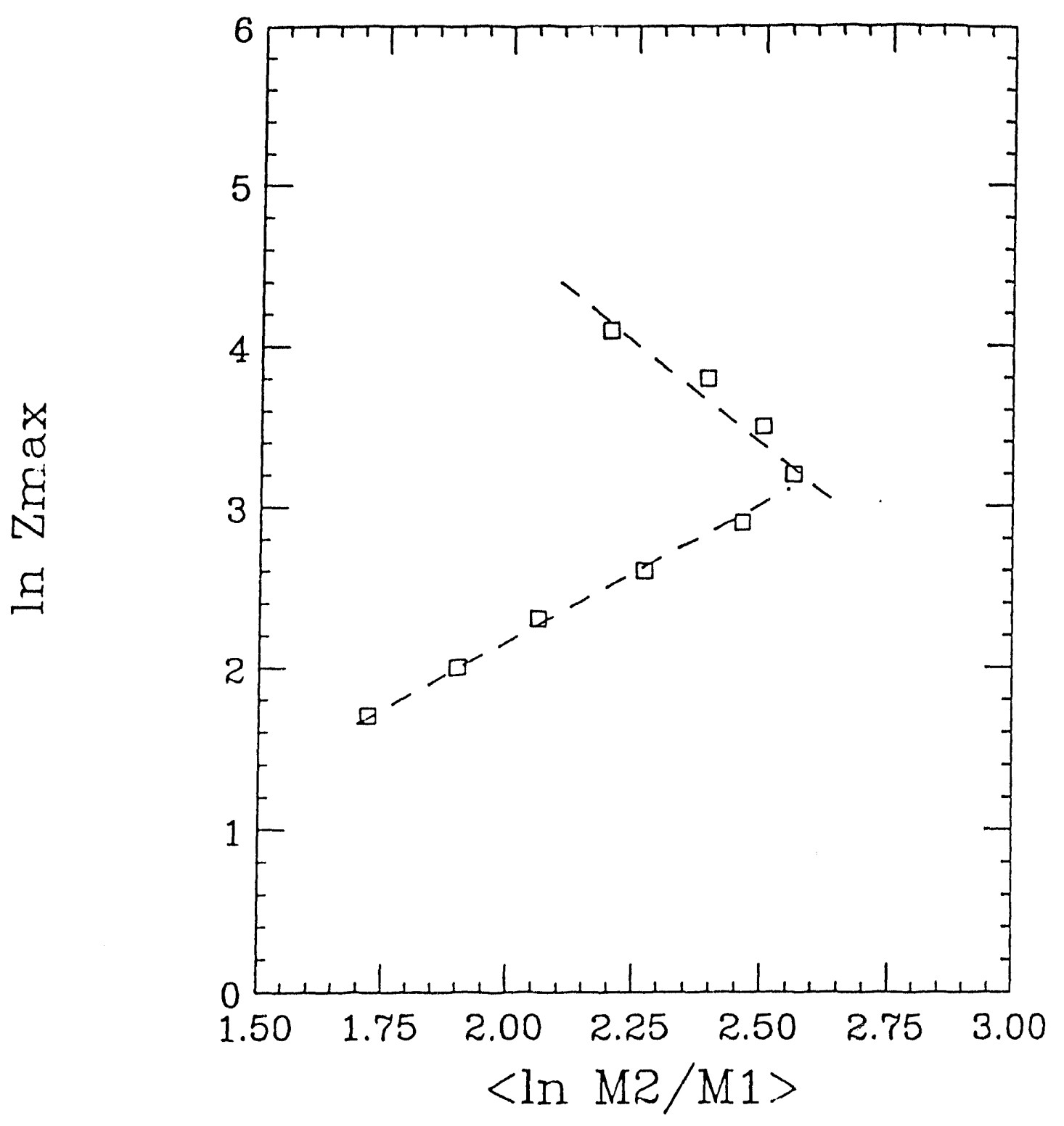

Figure 6.14 


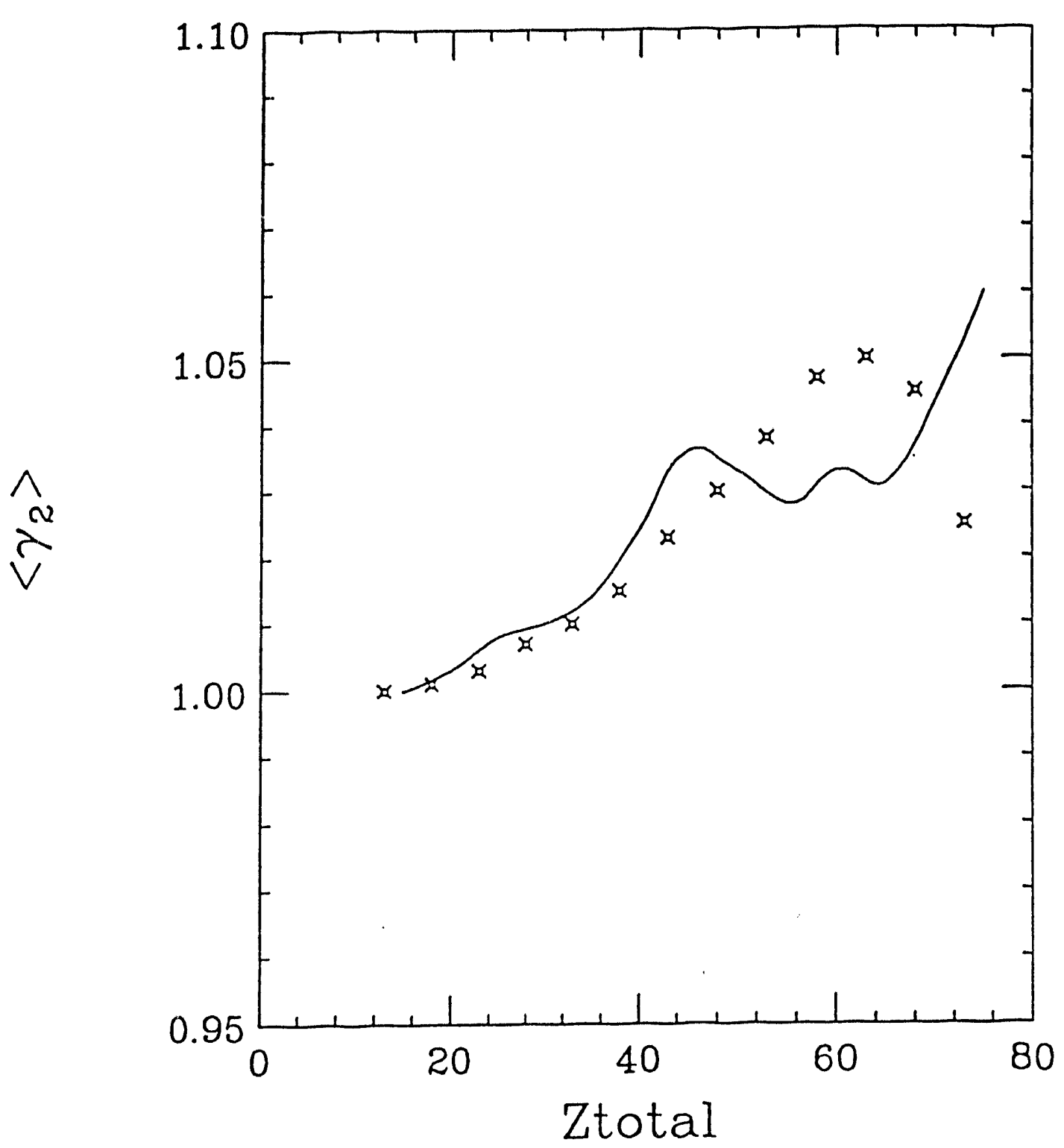

Figure 6.15 


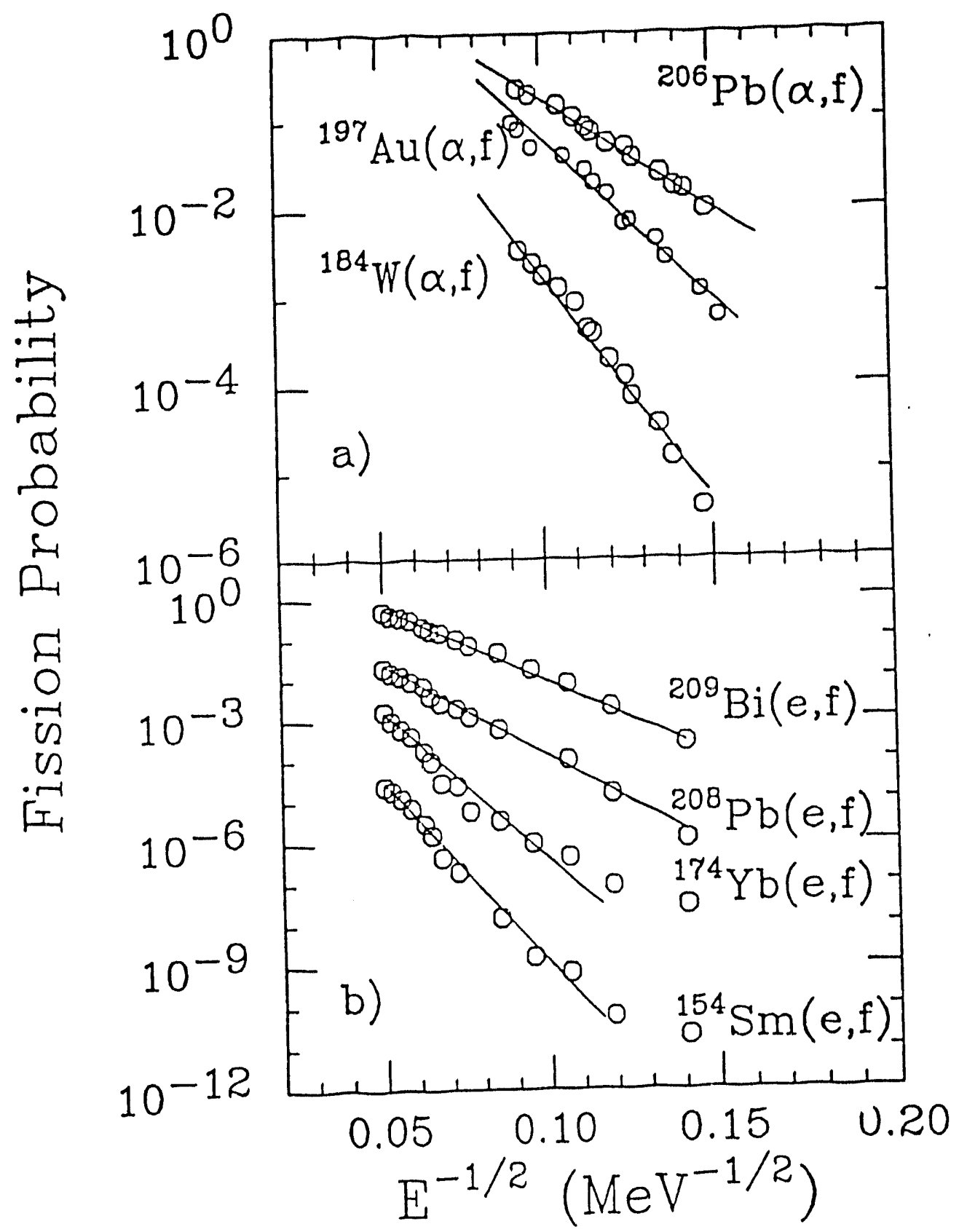

Figure 6.16 


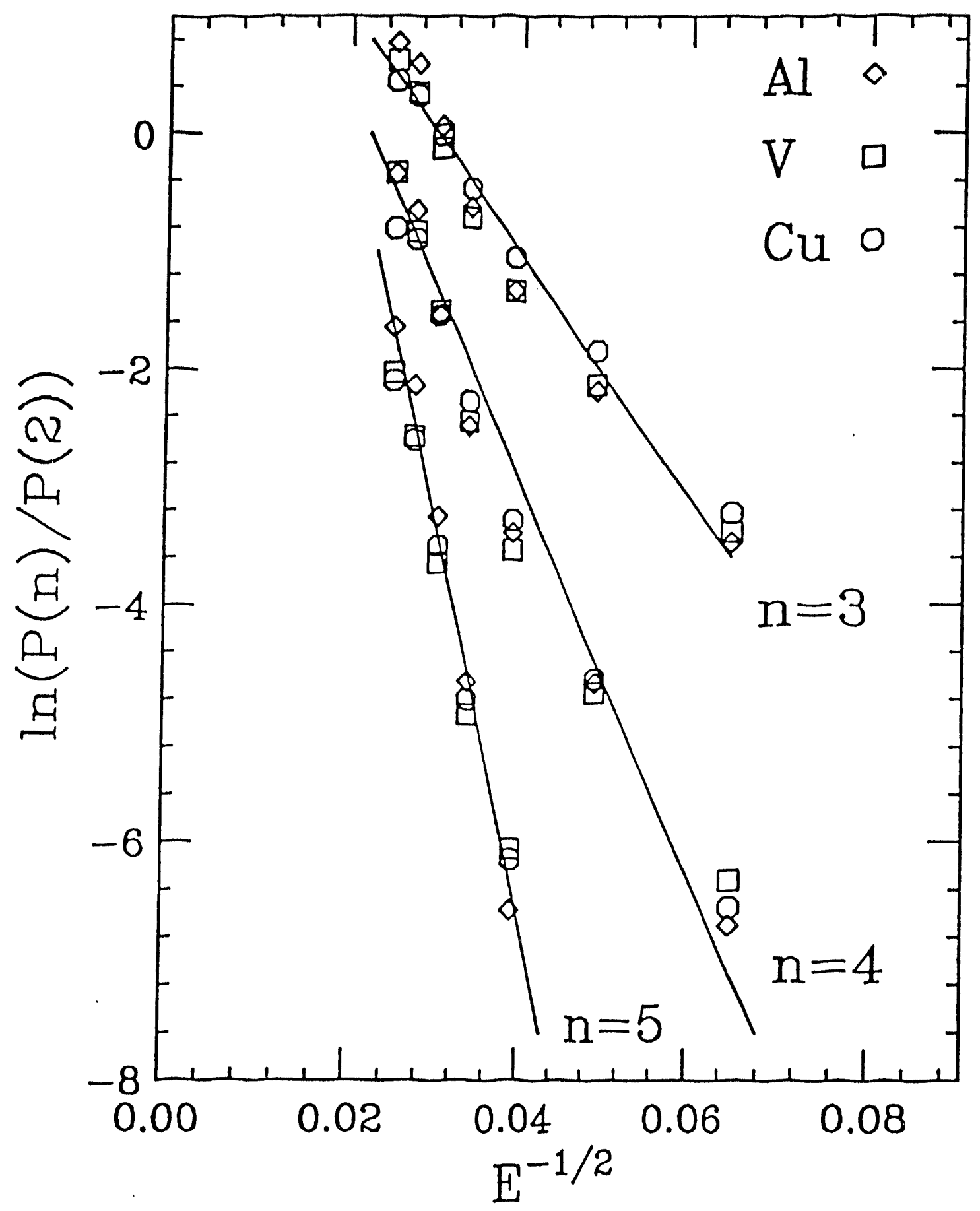

Figure 6.17 

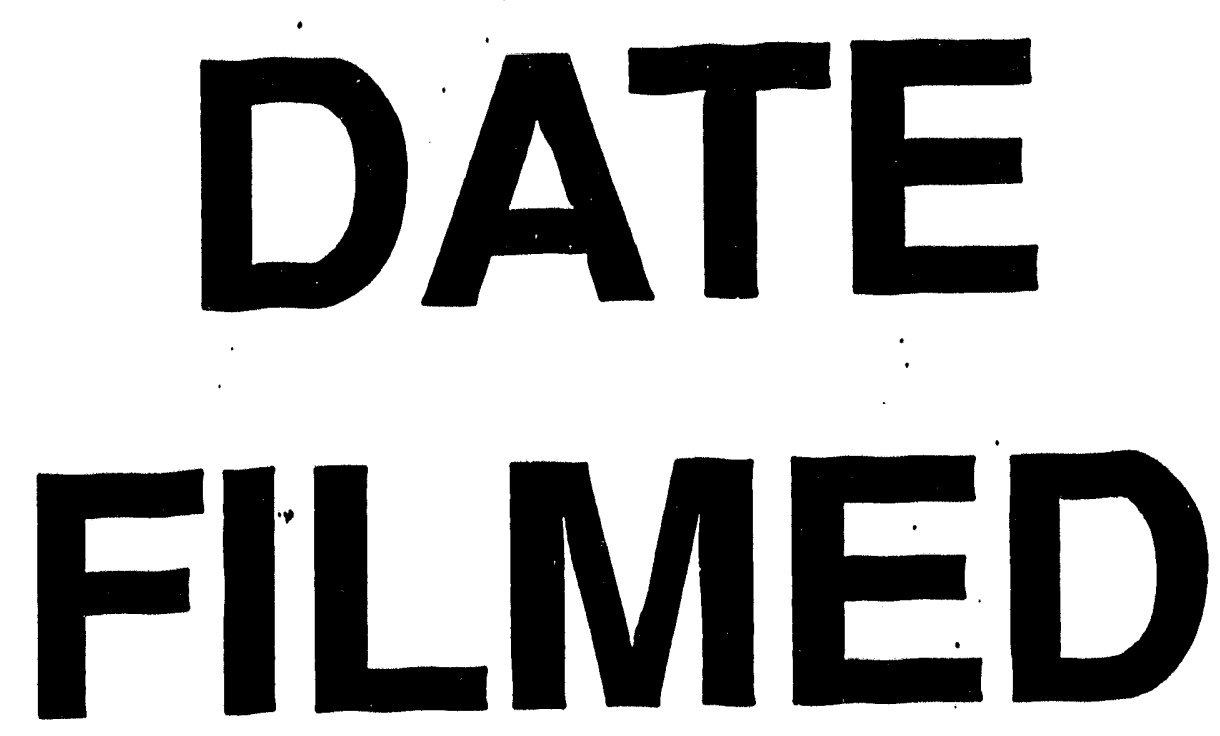

$1 / 5 / 94$
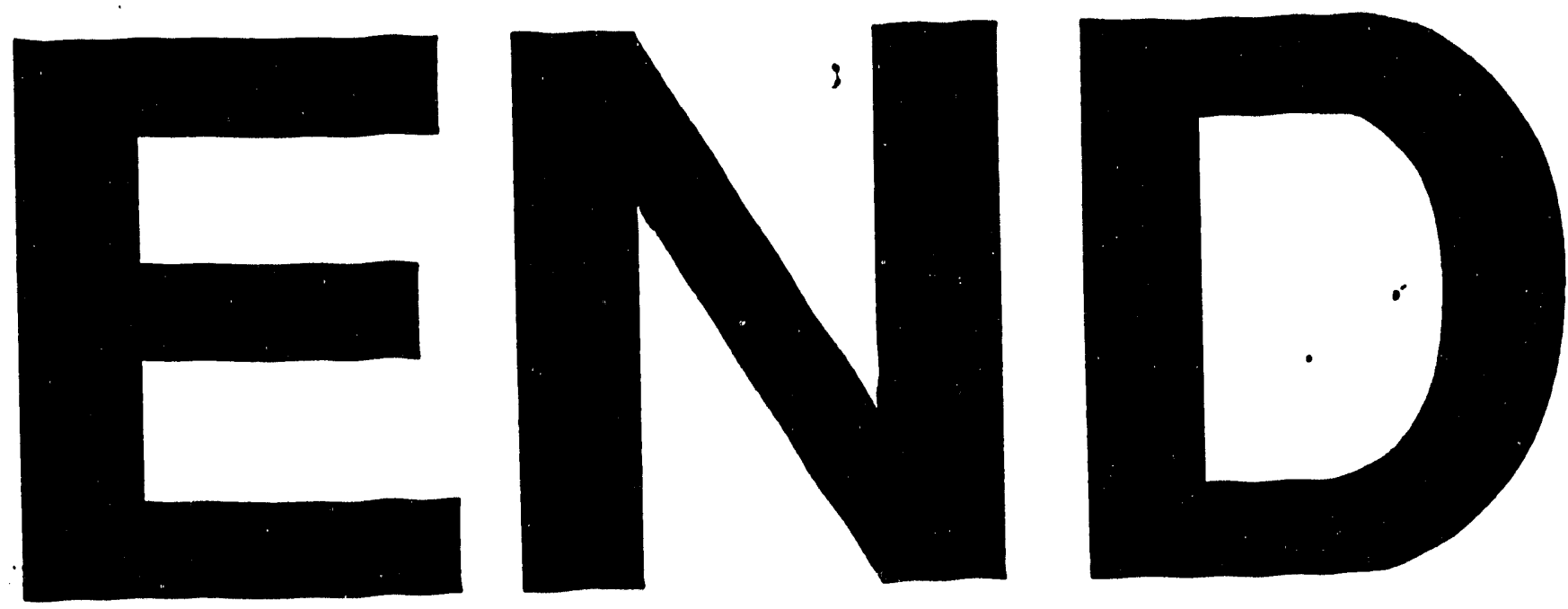


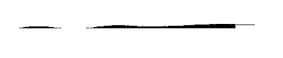

